\title{
Observing seismic attenuation in the Earth's mantle and inner core using normal modes
}

Sujania Asereth Talavera Soza

Utrecht Studies in Earth Sciences

No. 233 


\title{
Examination committee:
}

\author{
Prof. dr. C. A. Dalton
}

Department of Earth, Environmental and Planetary Sciences

Brown University, USA

Prof. dr. J. Ritsema

Department of Earth and Environmental Sciences

University of Michigan, USA

Prof. dr. B. Romanowicz

University of California, Berkeley, USA

Collège de France, Paris, France

Institut de Physique du Globe de Paris, France

Prof. dr. J. A. Trampert

Department of Earth Sciences

Universiteit Utrecht, The Netherlands

Dr. R. Widmer-Schnidrig

Black Forest Observatory

Universität Stuttgart, Germany

ISBN: 978-90-6266-598-3

DOI: https://doi.org/10.33540/738

Copyright (C) 2021 Sujania Asereth Talavera Soza, Utrecht, The Netherlands

Cover illustration: anelastic observations of upper mantle sensitive mode ${ }_{2} S_{12}$ and lower mantle sensitive mode ${ }_{1} S_{9}$, figure by SATS using Paraview (Ahrens et al., 2005).

The facilities of IRIS Data Services, and specifically the IRIS Data Management Centre, were used for access to waveforms, related metadata, and/or derived products used in this thesis. We also acknowledge the 'Global CMT project' webpage for the earthquake source parameters used in this thesis (Dziewonski et al., 1981; Dziewonski et al., 2012). GraphClick (http://www.arizona-software.ch/graphclick/) was used to digitalise data. Preand post-processing were done using ObsPy (Beyreuther et al., 2010) and FrosPy (Schneider et al., in prep.). The figures were generated using FrosPy and GMT (Wessel et al., 2013). 


\section{Observing seismic attenuation in the Earth's mantle and inner core using normal modes}

\section{Het observeren van seismische attenuatie in de aardmantel en de binnenkern met behulp van normal modes}

(met een samenvatting in het Nederlands)

Proefschrift

ter verkrijging van de graad van doctor aan de Universiteit Utrecht op gezag van de rector magnificus, prof. dr. H.R.B.M. Kummeling, ingevolge het besluit van het college voor promoties in het openbaar te verdedigen op

vrijdag 28 mei 2021 des middags te 4.15 uur

door

Sujania Asereth Talavera Soza

geboren op 28 november 1988 te Managua, Nicaragua 


\section{Promotor:}

Prof. dr. A. F. Deuss

This thesis was accomplished with financial support from the European Research Council (ERC) under the European Union's Horizon 2020 research and innovation programme (grant agreement No. 681535 - ATUNE) and a Vici award number 016.160.310/526 from the Netherlands organisation for scientific research (NWO). 
Para mis padres 
*Unwary readers should take warning that ordinary language undergoes modification to a high-pressure form when applied to the interior of the Earth; a few examples of equivalents follow:

High-pressure form:

certain

undoubtedly

positive proof

unanswerable argument

pure iron
Ordinary meaning:

dubious

perhaps

vague suggestion

trivial objection

uncertain mixture of all the elements

F. Birch (1952). Elasticity and constitution of the Earth's interior. Journal of Geophysical Research 57.2, pp. 227-286 


\section{Summary}

Seismic tomographic models based solely on wave velocities have limited ability to distinguish between a temperature or compositional origin for variations in Earth's structure. Attenuation or damping, which is the loss of energy as a wave travels through the Earth, is able to make that distinction because it is directly sensitive to temperature, partial melt and water content. In this thesis, we use whole Earth oscillations, or normal modes, to study $1 \mathrm{D}$ variations in inner core attenuation and $3 \mathrm{D}$ variations in mantle attenuation. Focussing and scattering, which are problematic when measuring seismic attenuation, are automatically included in our normal mode calculations when jointly modelling elastic and anelastic structure using first order perturbation theory.

For the inner core, we measure the controversial mode pair ${ }_{10} S_{2^{-} 11} S_{2}$, and find that our measurements indeed agree with a strongly attenuating inner core, just as seen in previous measurements of other inner core sensitive modes, and that our new observations can be used in future inner core tomographic studies. We attribute the previous disagreement to the strong energy exchange between ${ }_{10} S_{2}$ and ${ }_{11} S_{2}$, that changes the characteristics of the modes as a result of a $0.5 \%$ perturbation to either inner core shear velocity or radius. We also measure radial modes, both in isolation and resonating with other modes, to study bulk attenuation and inner core structure with an extended data set. We find indications of lower global bulk attenuation and lower inner core radial anisotropy than previously suggested.

For the mantle, the main focus of this thesis, we measure lateral variations in attenuation for both the upper and lower mantle. In the upper mantle, we find anti-correlation between velocity and attenuation, with strong attenuation found in regions with low seismic velocity. These results suggest a thermal origin for the low-velocity oceanic spreading ridges, agreeing with previous studies. In the lower mantle, we find the strongest attenuation in the 'ring around the Pacific' high velocity region, which is thought to be the 'graveyard' of subducted slabs, and not in the Large Low Shear Velocity Provinces (LLSVPs) under Africa and the Pacific. These findings might potentially be explained by either a small grain-size in combination with cold temperatures in the slabs resulting in strong attenuation, or the presence of the strongly attenuating post-perovskite mineral in the slab regions. The weakest lower mantle attenuation is found near Hawaii and southern Africa, at the edges of the LLSVPs. This suggests that these two low attenuation regions might be dominated by compositional variations instead of temperature, potentially due to iron enrichment in agreement with larger density found in the same places. 



\section{Samenvatting}

Seismische tomografische modellen die uitsluitend gebaseerd zijn op golfsnelheid kunnen geen onderscheid maken tussen temperatuur of compositie als oorzaak van variaties in de structuur van de aarde. Attenuatie of demping, i.e. het verlies van energie als een golf door de aarde reist, is in staat om dat onderscheid wel te maken omdat het direct gevoelig is voor temperatuur, partiële smelt en watergehalte. In dit proefschrift gebruiken we de eigentrillingen van de aarde, ook wel normal modes genoemd, om 1D-variaties in demping in de binnenkern en 3D-variaties in demping in de mantel te bestuderen. Focussing en verstrooiing, die problematisch zijn bij het meten van seismische demping, worden automatisch meegenomen in onze normal mode berekeningen doordat de elastische en anelastische structuur gezamenlijk gemodelleerd worden met gebruik van eerste orde storingstheorie.

Voor de binnenkern, hebben we het controversiële normal mode paar ${ }_{10} S_{2^{-}}{ }_{11} S_{2}$ bestudeerd, en vinden we dat onze metingen inderdaad overeenkomen met een sterk dempende binnenkern, net als in eerdere metingen van andere binnenkern gevoelige modes. Onze nieuwe waarnemingen kunnen gebruikt worden in toekomstige tomografische inversies van de binnenkern. We schrijven het meningsverschil over de demping van deze modes in eerdere studies toe aan de sterke energie-uitwisseling tussen ${ }_{10} S_{2}$ en ${ }_{11} S_{2}$, wat de eigenschappen van de modes verandert als gevolg van een $0.5 \%$ verandering van de straal of de transversale golfsnelheid van de binnenkern. We hebben ook radiale modes gemeten, zowel geïsoleerd als resonerend met andere modes, met als doel om een uitgebreidere dataset te verkrijgen voor het bestuderen van de bulkdemping en structuur van de binnenkern. We vinden aanwijzingen voor een lagere globale bulkdemping en een lagere radiale anisotropie van de binnenkern dan eerder gesuggereerd.

Voor de mantel, het belangrijkste onderwerp van dit proefschrift, meten we laterale variaties in demping voor zowel de boven- als de ondermantel. In de bovenmantel vinden we anti-correlatie tussen snelheid en demping, met andere woorden een sterke demping in gebieden met een lage seismische snelheid. Deze resultaten suggereren een thermische oorsprong voor de lage snelheid van de oceanische spreidingsruggen, in overeenstemming met eerder onderzoek. In de ondermantel vinden we de sterkste demping in de 'ring rondom de Stille Oceaan' die een hoge snelheid heeft, en niet in de Large Low Shear Velocity Provinces (LLSVPs) onder Afrika en de Pacifische Oceaan die een lage snelheid hebben. Deze bevindingen kunnen mogelijk verklaard worden door of een kleinere korrelgrootte in combinatie met lage temperaturen in de 
gesubduceerde platen wat resulteert in sterke damping, of door de aanwezigheid van het sterk dempende post-perovskiet mineraal in deze regio's. De zwakste demping van de ondermantel wordt gevonden in de buurt van Hawaii en het zuidelijke deel van Afrika, die zich aan de randen van de LLSVPs bevinden. Dit suggereert dat deze twee regio's gedomineerd zouden kunnen zijn door variaties in compositie in plaats van in temperatuur, mogelijk als gevolg van ijzerverrijking, wat overeenstemt met een grotere dichtheid die ook op dezelfde plaatsen gevonden wordt. 


\title{
Zusammenfassung
}

\author{
Seismische Dämpfung im Erdmantel und im inneren Kern mit Hilfe von \\ Eigenmoden der Erde.
}

Tomographiemodelle, die ausschließlich durch seismische Wellengeschwindigkeiten erstellt wurden, können nur bedingt erklären ob der Unterschied in der Struktur der Erde auf thermische oder chemische Anomalien zurückzuführen ist. Bei diesem Problem kann die seismische Dämpfung, die den Energieverlust einer seismischen Welle durch die Erde beschreibt, helfen, da sie direkt von der Temperatur, der partiellen Schmelze und dem Wassergehalt abhängig ist. In dieser Doktorarbeit verwenden wir Eigenschwingungen der Erde, oder Eigenmoden, um die eindimensionale Dämpfungsstruktur des inneren Kerns und die dreidimensionale Dämpfungsstruktur des Mantels zu untersuchen. Fokussierung und Streuung von seismischen Wellen, die bei der Messung der seismischen Dämpfung problematisch sind, sind inhärent Teil unserer Eigenmoden, die wir für die elastische und anelastische Struktur mithilfe der Störungstheorie erster Ordnung berechnen.

Für den inneren Kern messen wir das kontrovers diskutierte Modenpaar ${ }_{10} S_{2-11} S_{2}$ und können zeigen, dass unsere Messungen mit einem stark dämpfenden inneren Kern übereinstimmen, wie auch schon in früheren Messungen von Moden mit hoher Sensitivität des inneren Kerns. Außerdem können unsere neuen Ergebnisse für neue tomographische Modelle des inneren Kerns verwendet werden. Wir führen die Kontroverse auf den starken Energieaustausch zwischen ${ }_{10} S_{2}$ und ${ }_{11} S_{2}$ zurück. Dieser Austausch verändert die Eigenschaften der Moden entweder durch eine $0.5 \%$ ige Störung der Scherwellengeschwindigkeit oder des Radius des inneren Kerns. Wir messen auch radiale Moden, sowohl isoliert als auch in Resonanz mit anderen Moden, um die Durchschnitts-Dämpfung und die Struktur des inneren Kerns mit einem erweiterten Datensatz zu untersuchen. Unserer Ergebnisse weisen auf eine geringere globale Durschnitts-Dämpfung und eine geringere radiale Anisotropie des inneren Kerns als bisher angenommen hin.

Im Erdmantel, den Schwerpunkt dieser Arbeit, messen wir laterale Variationen der Dämpfung sowohl für den oberen als auch den unteren Mantel. Im oberen Mantel, finden wir eine Anti-Korrelation zwischen seismischer Wellengeschwindigkeit und Dämpfung, wobei starke Dämpfung in Regionen mit niedriger seismischer Wellengeschwindigkeit auftritt. Diese Ergebnisse deuten auf einen thermischen Ursprung für Mittelozeanische Rücke mit niedriger seismischer Wellengeschwindigkeit 
hin und stimmen mit früheren Studien überein. Im unteren Mantel, sehen wir die stärkste Dämpfung in Regionen mit höheren seismischen Wellengeschwindigkeiten rund um den Pazifik, die als „Friedhof“, subduzierter tektonischer Platten angesehen werden. Wir sehen die stärkste Dämpfung nicht in den Large Low Shear Velocity Provinces (LLSVPs) unter Afrika und dem Pazifik. Diese Ergebnisse lassen sich möglicherweise durch kleine Korngrößen in Kombination mit niedrigeren Temperaturen in den tektonischen Platten erklären, die zu einer starken Dämpfung führen, oder durch das Vorhandensein des stark dämpfenden Post-Perowskit-Minerals. Die schwächste Dämpfung des unteren Mantels findet sich in der nähe von Hawaii und Süd-Afrika, an den Rändern der LLSVPs. Dies deutet darauf hin, dass diese Regionen mit geringer Dämpfung, von chemischen Anomalien anstatt von Temperaturanomalien dominiert werden, was mit Eisenanreicherungen, die mit der höheren Dichte dort übereinstimmt, erklärt werden kann. 


\section{Resumen}

\section{Observaciones de atenuación sísmica en el manto y núcleo interno de la Tierra utilizando modos normales}

Modelos tomográficos sísmicos basados únicamente en velocidades de onda tienen limitaciones para distinguir entre un origen térmico o composicional para las variaciones estructurales de la Tierra. La atenuación o amortiguación sísmica (es decir, la pérdida de energía a medida que una onda sísmica viaja a través de la Tierra) es capaz de hacer esta distinción, ya que es directamente sensible a la temperatura, a la fusión parcial y al contenido de agua. En esta tesis, utilizamos las oscilaciones de la Tierra a escala planetaria, o modos normales, para estudiar las variaciones 1D en la atenuación del núcleo interno y las variaciones $3 \mathrm{D}$ en la atenuación del manto. El enfoque y el esparcimiento, que son efectos problemáticos al medir la atenuación sísmica, se incluyen automáticamente en los cálculos de modos normales, ya que las estructuras elástica y anelástica se pueden modelar de manera conjunta utilizando la teoría de perturbación de primer orden.

Para el núcleo interno, medimos el controversial par de modos normales ${ }_{10} S_{2-11} S_{2}$, y encontramos que nuestras mediciones en efecto están de acuerdo con un núcleo interno fuertemente atenuante, tal como se ha visto en anteriores mediciones de otros modos normales sensibles al núcleo interno, y que nuestras nuevas observaciones pueden ser utilizadas en futuros estudios tomográficos del núcleo interno. Atribuimos el anterior desacuerdo al fuerte intercambio de energía entre los modos ${ }_{10} S_{2}$ y ${ }_{11} S_{2}$, el cual cambia las características de estos modos como resultado de una perturbación de $0.5 \%$ en la velocidad de onda de corte o en el radio del núcleo interno. También medimos los modos radiales, tanto de forma aislada como en resonancia con otros modos normales, para estudiar la atenuación de volumen y la estructura del núcleo interno utilizando una base de datos más extensa. Encontramos indicios de una menor atenuación global de volumen y una menor anisotropía radial del núcleo interno con respecto a lo que se había sugerido anteriormente.

Para el manto, el tema principal de esta tesis, medimos las variaciones laterales de la atenuación tanto para el manto superior como para el inferior. En el manto superior, encontramos una anticorrelación entre la velocidad y la atenuación, es decir una fuerte atenuación en regiones con baja velocidad sísmica. Estos resultados sugieren un origen térmico para las dorsales mediooceánicas de baja velocidad, lo que concuerda con estudios anteriores. En el manto inferior, encontramos la atenuación 
más fuerte en el "cinturón alrededor del Pacífico" el cual también muestra alta velocidad y se considera el "cementerio" de placas tectóticas, y no en las grandes provincias de baja velocidad de onda de corte (LLSVPs en inglés) bajo África y el Pacífico. Estos hallazgos podrían ser potencialmente explicados ya sea por una combinación de bajas temperaturas y un tamaño pequeño de grano, o por la presencia del fuertemente atenuante mineral post-perovskita en estas mismas regiones. La atenuación más débil del manto inferior se encuentra cerca de Hawai y el sur de África, en los bordes de las LLSVPs. Esto sugiere que estas dos regiones de baja atenuación podrían estar dominadas por variaciones de composición en lugar de temperatura, potencialmente debido al enriquecimiento de hierro, lo cual es consistente con el incremento de densidad encontrado en estudios previos en las mismos regiones. 


\section{Contents}

Summary vii

Samenvatting $\quad$ ix

Zusammenfassung $\quad$ xi

Resumen $\quad$ xiii

1 Introduction 1

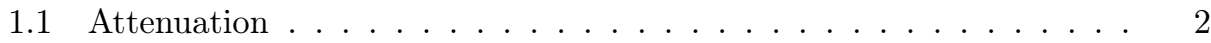

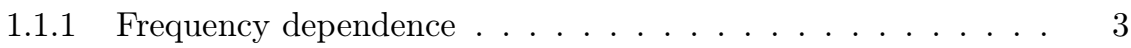

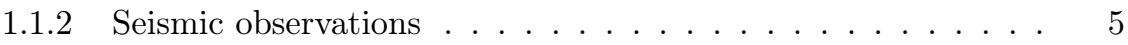

1.1.3 Mineral physics . . . . . . . . . . . . . . . . . . 10

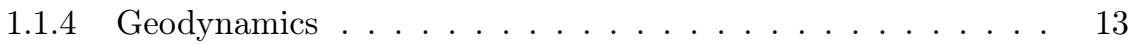

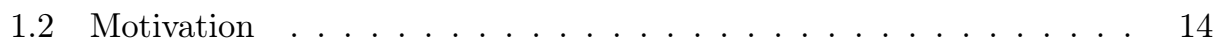

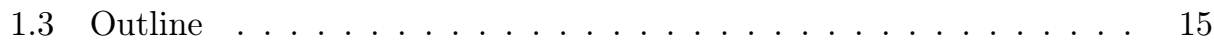

2 Method and Data $\quad 19$

2.1 Normal modes and splitting functions . . . . . . . . . . . . . 20

2.1.1 Normal mode definition . . . . . . . . . . . . . . . . 20

2.1.2 Splitting functions .................. . . . . . . . . . . . . . . 22

2.1.3 Synthetic splitting functions . . . . . . . . . . . . . . . . . 23

2.2 Synthetic seismograms . . . . . . . . . . . . . . . 24

2.2.1 Coriolis and kinetic energy approximation . . . . . . . . . 25

2.2 .2 Splitting matrix . . . . . . . . . . . . . . 26

2.2.3 Linearising the problem: calculating derivatives . . . . . . . . 28

2.3 Inverse problem . . . . . . . . . . . . . . . . . . . . . 29

2.3 .1 Uncertainties . . . . . . . . . . . . . . . . . . . . . . . . . . . . .

2.3.2 F-tests ...................... 30

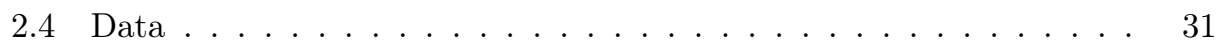

2.A Appendix: Exact momentum equation ............. 37 
3 Constraining 1D inner core attenuation using strongly coupled modes

3.1 Introduction . . . . . . . . . . . . . . . . . . . . 40

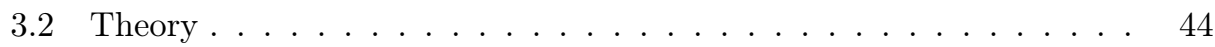

3.3 Method and Data . . . . . . . . . . . . . . . . . . . . . 45

3.4 Results.............................. 45

$3.4 .1{ }_{10} S_{2-11} S_{2} \ldots \ldots \ldots \ldots \ldots \ldots$

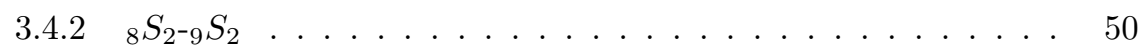

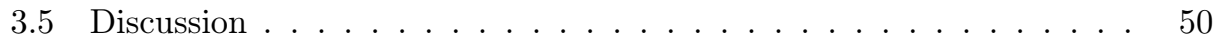

3.5.1 Inner core radius and shear wave velocity . . . . . . . . 50

3.5.2 Attenuation ...................... 53

3.5.3 Innermost inner core anisotropy . . . . . . . . . . . . 54

3.6 Conclusions . . . . . . . . . . . . . . . . . . . . . 55

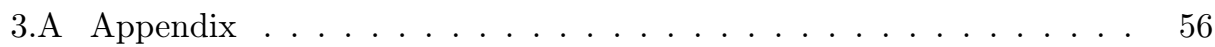

3.A.1 ${ }_{8} S_{2-9} S_{2} \ldots \ldots \ldots \ldots \ldots \ldots$. . . . . . . . . . . . . 56

3.A.2 Comparison between MINEOS and first order perturbation theory 57

4 Observing long-period radial modes $\quad 59$

4.1 Introduction . . . . . . . . . . . . . . . . . . 60

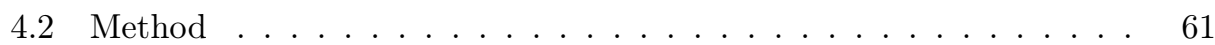

4.3 Data . . . . . . . . . . . . . . . . . . 62

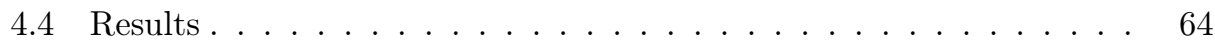

4.4.1 Self-coupling approach (SC) . . . . . . . . . . . . . 64

4.4 .2 Group-coupling approach $(\mathrm{GC}) \ldots \ldots 6$

4.5 Interpretation . . . . . . . . . . . . . . . . 76

4.5.1 Bulk attenuation . . . . . . . . . . . . . 76

4.5.2 Radial anisotropy in the inner core . . . . . . . . . . . . 78

4.5.3 Inner core cylindrical anisotropy . . . . . . . . . . . . . . . 80

4.6 Conclusions . . . . . . . . . . . . . . . . . . . . . 82

5 Synthetic tests for 3D variations in mantle attenuation $\quad \mathbf{8 3}$

5.1 Introduction . . . . . . . . . . . . . . . . . 84

5.2 Forward modelling . . . . . . . . . . . . . . . . . . . 87

5.2.1 Scaling relationship between $q_{\mu}$ and $v_{s} \ldots \ldots . \ldots 87$

5.2 .2 Full coupling synthetics . . . . . . . . . . . . . . . 89

5.2 .3 Individual mode sensitivity to 3D mantle $q_{\mu} \ldots \ldots$. . . . . 91

5.3 Synthetic anelastic splitting function tests . . . . . . . . . . . . . 94

5.3.1 Synthetic anelastic splitting function recovery . . . . . . . . 95

5.3.2 $M_{0}$ variation effect on anelastic splitting functions . . . . . 97

5.4 Conclusion ......................... 102

5.A Appendix: $R_{q}$ ratio variations ................ 104 
6 Constraining 3D mantle attenuation 109

6.1 Introduction . . . . . . . . . . . . . . . . . . . 110

6.1.1 Attenuation from mineral physics . . . . . . . . . . . . . 110

6.1.2 Attenuation from seismology . . . . . . . . . . . . . 113

6.2 Method and Data . . . . . . . . . . . . . . . . 115

6.2 .1 Damping ....................... 116

6.2.2 Influence of the starting model . . . . . . . . . . . . . . . 116

6.2 .3 Normal mode selection . . . . . . . . . . . . . . . . . 117

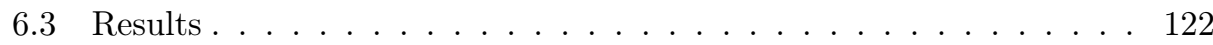

6.3.1 Fundamentals and $1^{\text {st }}$ overtone modes . . . . . . . . . . . 124

6.3.2 $2^{\text {nd }}$ and $3^{\text {rd }}$ overtone modes . . . . . . . . . . . . . . . 130

6.3.3 Cross-coupled splitting functions . . . . . . . . . . . . . 132

6.4 Interpretation . . . . . . . . . . . . . . . . . . . . . . 134

6.4.1 Upper mantle . . . . . . . . . . . . . . . . . . . 134

6.4 Lower mantle . . . . . . . . . . . . . . . . . . . . 139

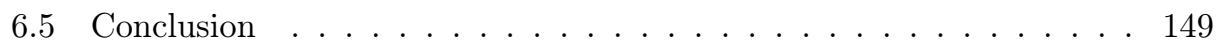

6.A Appendix: $c_{s t}+d_{s t}$ damping curves . . . . . . . . . . . . . 150

6.B Appendix: $d_{s t}$ observations at larger structural degrees . . . . . . . . 152

7 Synthesis $\quad \mathbf{1 5 5}$

7.1 General challenges in this thesis . . . . . . . . . . . . . . . 157

7.2 Future research directions . . . . . . . . . . . . . . . . 158

$\begin{array}{lc}\text { Bibliography } & 163\end{array}$

$\begin{array}{ll}\text { Abbreviations } & \mathbf{1 7 5}\end{array}$

$\begin{array}{lr}\text { Acknowledgements } & 177\end{array}$

$\begin{array}{ll}\text { Curriculum Vitae } & 181\end{array}$ 



\section{Introduction}

Seismology is the study of elastic waves, generated by earthquakes, as they travel through the Earth and are recorded by seismometers at the Earth's surface. The first global network of seismometers was built in 1962. Since then, the network's infrastructure has been expanded and its data used to study our planet. Many advancements have been made thereafter, one of them being our understanding of Earth's structure through tomographic models. These models map velocity variations, both lateral and with depth, using seismic waves. Current global 1D and 3D velocity models show very good agreement with each other, even when built using data types across different frequency bands. This is especially noticeable for large scale shear wave velocity structure, which we believe is best constrained.

However, it is still unclear if the cause of these velocity variations is mainly thermal, or if compositional heterogeneity, such as variations in iron, mineral phase or water content, is also needed. In order to answer this question, we need additional information from seismology, such as $3 \mathrm{D}$ variations in density or attenuation. Unfortunately, achieving the same advancements as in velocity tomography has been much more difficult when measuring attenuation or damping, which is the loss or absorption of energy of a seismic wave as it travels the Earth. Although attenuation is much less studied than velocity in seismology, it determines the time scales of anelastic physical phenomena and, without it, seismic waves would keep travelling along the Earth forever. Also, attenuation is directly sensitive to variations in temperature and water content, and thus could help distinguish between a thermal or compositional origin of the velocity variations.

Using the massive amount of data available nowadays, seismologists often show the recurrence of earthquakes as a function of their magnitude and equivalent energy release, and compare them to other natural and man-made disasters. Images like Fig. 1.1 illustrate just how much energy is released by an earthquake and the power of destruction it holds. We often discuss how this energy destroys cities and causes massive damage to our lives. However, we seldom consider how this energy is also attenuated or absorbed, which mechanisms of relaxation are involved or how they can be related to the minerals from which our planet is made. In this thesis, we will use seismology to try to find a link between where earthquake energy is attenuated and the seismic and mineralogical structure within the Earth in order to improve our understanding of the inner workings of our planet. 


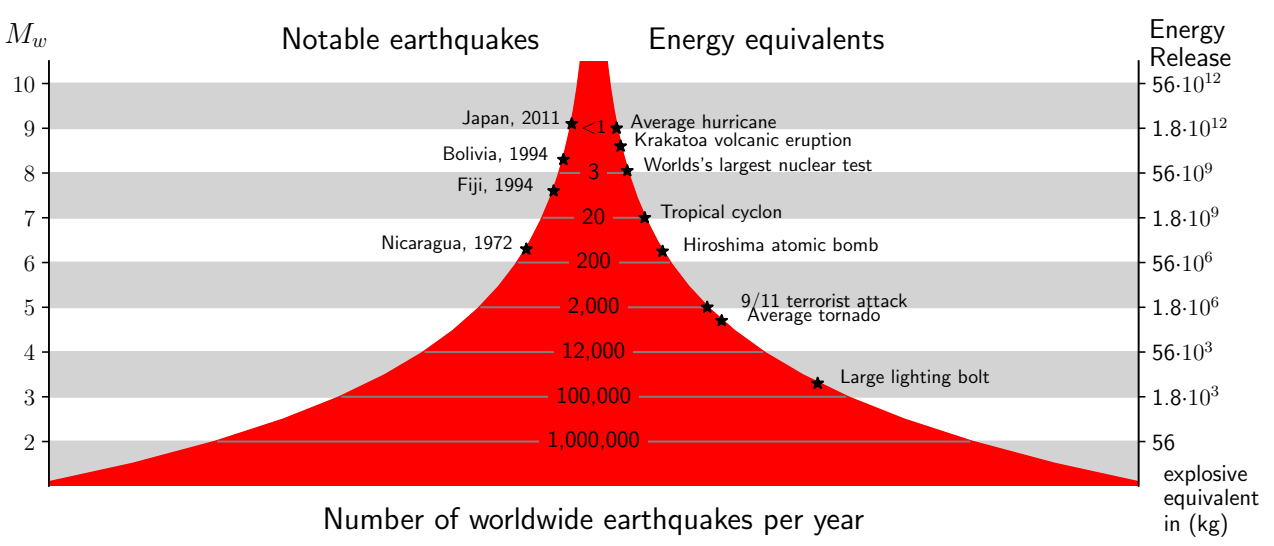

Figure 1.1: Modified from the IRIS website (https://www.iris.edu/). The number of average earthquakes per year are plotted against their moment magnitude $M_{w}$ and their equivalent energy release $E$. Historic earthquakes are highlighted together with notable natural and man-made disasters.

\subsection{Attenuation}

Seismic attenuation $q$ or damping is the transformation of seismic energy into heat as waves travel through the Earth and quantifies loss of energy. It is measured in terms of $q=\frac{1}{Q}$, where $Q$ is the 'quality factor', which describes the decay in amplitude per seismic oscillation. The quality factor is defined as

$$
Q=2 \pi\left(\frac{E_{\max }}{\delta E}\right)
$$

where $E_{\max }$ is the maximum elastic energy within a cycle. An alternative definition requires replacing the maximum energy by the average stored energy, as well as replacing 2 by 4 in Eq. 1.1 (e.g. O'Connell and Budiansky, 1978). The particular definition above allows us to also define $Q$ as the ratio between the real and imaginary part of the complex elastic modulus (e.g. Romanowicz and Mitchell, 2015).

Attenuation is observed as a decrease in the amplitude of seismic waves. It is difficult to measure attenuation because elastic effects, such as focusing and scattering, also decrease or increase seismic amplitudes (Stein and Wysession, 2009). Focussing is a local amplification of energy due to lateral velocity variations and scattering is a redistribution of energy due to small scale velocity heterogeneities. Both distort the recorded seismic signal, but energy is not lost. Instead, focussing and scattering merely redistribute energy to another location and some of it may arrive later as seismic coda. This means that separating the 'apparent attenuation', displayed by elastic effects of focussing and scattering, from actual dissipation or 'intrinsic attenuation' due to anelastic loss of energy is of importance when making seismic observations of attenuation.

Below we will discuss previous seismic observations of attenuation and also the anelasticity of mantle and inner core minerals that are relevant for this thesis. For a deeper understanding of seismic attenuation and its relevant laboratory experiments 


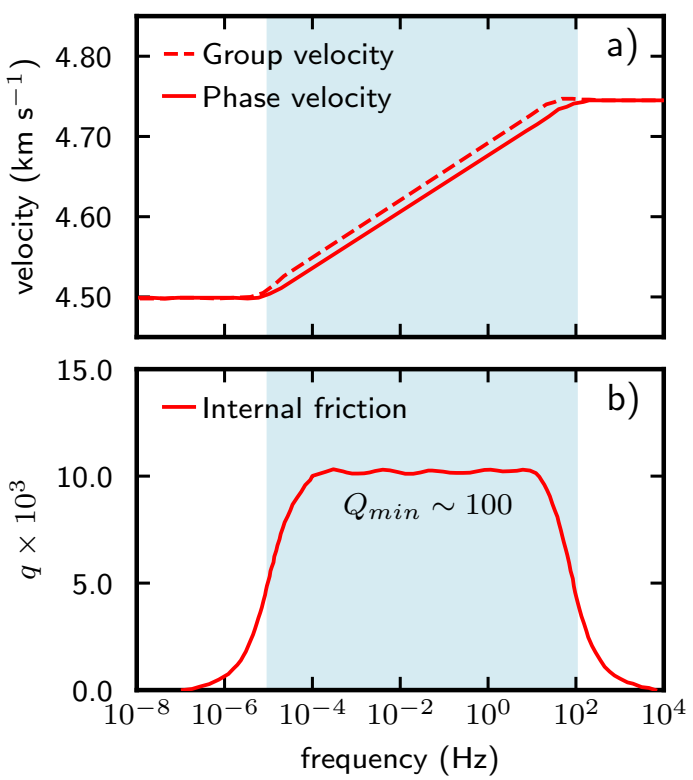

Figure 1.2: Modified from Liu et al. (1976). Schematic model calculated for a linear solid consisting of twelve superposed relaxation mechanisms with different compositions and at different pressures and temperatures. The model is presented in terms of its (a) group and phase velocity and (b) internal friction $q$. The superposition of the relaxation mechanisms yields a roughly constant $Q=1 / q$ over a wide range of frequencies. The area where anelastic behaviour occurs is presented in light blue, between the low cut-off frequency $10^{-5}$ and the high cut-off frequency $10^{2}$, leading to the 'absorption band' model.

see the more in-depth reviews of Romanowicz and Durek (2000), Romanowicz and Mitchell (2015), Jackson (2015), and Faul and Jackson (2015).

\subsubsection{Frequency dependence}

An important consequence of the Earth being an attenuating medium is physical dispersion, which causes a wave's intrinsic velocity to vary with frequency (Fig. 1.2a). In general, dispersion results in wave velocity being lower at lower frequency. This is why velocity models depend on the type of data used in their construction, with the same seismic anomalies showing lower velocities for low frequency normal modes than for high frequency body waves. Physical dispersion is needed to explain 1D and 3D velocity measurements across large frequency ranges, and taking it into account allows different data types to be united in a single model (e.g. Dziewonski and Anderson, 1981). Dispersion also allows us to make relevant comparisons between velocity models and other geophysical observations, such as the geoid (e.g. Romanowicz, 1990). However, this is a two-way street, because attenuation structure must be known in order to determine the dispersion caused by dissipation. 
In the 1960s, seismic measurements and early laboratory experiments suggested that attenuation was only weakly dependent on frequency (Knopoff, 1964). This contradicted the rather strong frequency dependence shown by single relaxation mechanisms, which are modelled using standard linear solids ${ }^{1}$. This inconsistency was reconciled by Liu et al. (1976) through the 'absorption band' model, which was build using a standard linear solid consisting of twelve superposed thermally activated microscopic relaxation mechanisms that envelop the entire observed absorption band. In the most simple version of the 'absorption band' model (Fig. 1.2b), attenuation $q$ is non-zero within a specific frequency band. The superposition of the relaxation mechanisms yields a roughly constant $Q=1 / q$ over a wide range of frequencies within the band and is only strongly frequency dependent at the edges of the absorption band. However, we expect the frequency dependence of attenuation to be stronger for the real Earth at all frequencies (see Fig. 1.3). This stronger dependency can be modelled with a mildly frequency dependent model using power laws of the form $q \propto \omega^{-\alpha}$ within the absorption band, where $\alpha$ is a model dependent parameter that has values below 0.5 (Anderson and Minster, 1979; Anderson and Given, 1982). Attenuation measurements, going from the Chandler Wobble ${ }^{2}$ occurring at sub-seismic frequencies (e.g. Benjamin et al., 2006) to high frequency body waves traversing the whole mantle (e.g. Sipkin and Jordan, 1980), show only weak frequency dependence for attenuation. All seismic measurements are in agreement with an absorption band model, and constrain $\alpha$ to be between 0.2 and 0.4 (Romanowicz and Mitchell, 2015).

More recently, Lekić et al. (2009) studied the behaviour of $\alpha$ for a frequency band of $0.3-5 \mathrm{mHz}$, using the $Q$ values of a range of modes and surface waves (Fig. 1.3). For frequencies above $5 \mathrm{mHz}$ they derived $\alpha=0.3$, while for frequencies between $3.3 \mathrm{mHz}$ and $1.25 \mathrm{mHz}$ they obtained $\alpha=0.1$. But, most remarkably they found $\alpha=-0.4$ for frequencies below $1 \mathrm{mHz}$, which means attenuation increases with decreasing frequency for the lowest frequency modes. This conclusion was further explored by Lau and Faul (2019), because it contradicted a low frequency $\alpha$ model based on various body tides and the Chandler Wobble, which spanned frequencies between $0.02 \mathrm{mHz}$ and $1.7 \times 10^{-6} \mathrm{mHz}$ (Benjamin et al., 2006). The model of Benjamin et al., 2006 has a positive $\alpha$ of 0.2 for frequencies below $0.3 \mathrm{mHz}$ instead of the negative $\alpha$ proposed by Lekić et al. (2009) (Fig. 1.3). Using both low frequency normal modes $(0.3-2.1 \mathrm{mHz})$ and tidal waves, Lau and Faul (2019) reconciled the $\alpha$ models obtained by Lekić et al. (2009) and Benjamin et al. (2006). Based on synthetic modelling, Lau and Faul (2019) propose that low frequency normal modes lie in a plateau of constant $\alpha$, which changes into positive $\alpha$ for even lower frequency tidal waves. The transition between constant and positive $\alpha$ value, may occur directly or through an inflexion point with negative $\alpha$, explaining the observation of Lekić et al. (2009).

Furthermore, it is important to realise that because data at different frequencies is sensitive to different depths in the Earth, the exact value of $\alpha$ may also be altered by variations of attenuation with depth. This trade-off between the effects of

${ }^{1}$ Standard linear solids describe the behaviour of viscoelastic materials using a linear combination of springs and dashpots that represent the elastic and anelastic response of the material.

${ }^{2}$ The Chandler Wobble is a small latitude variation of the Earth's rotation axis with a period of around 14 months. 


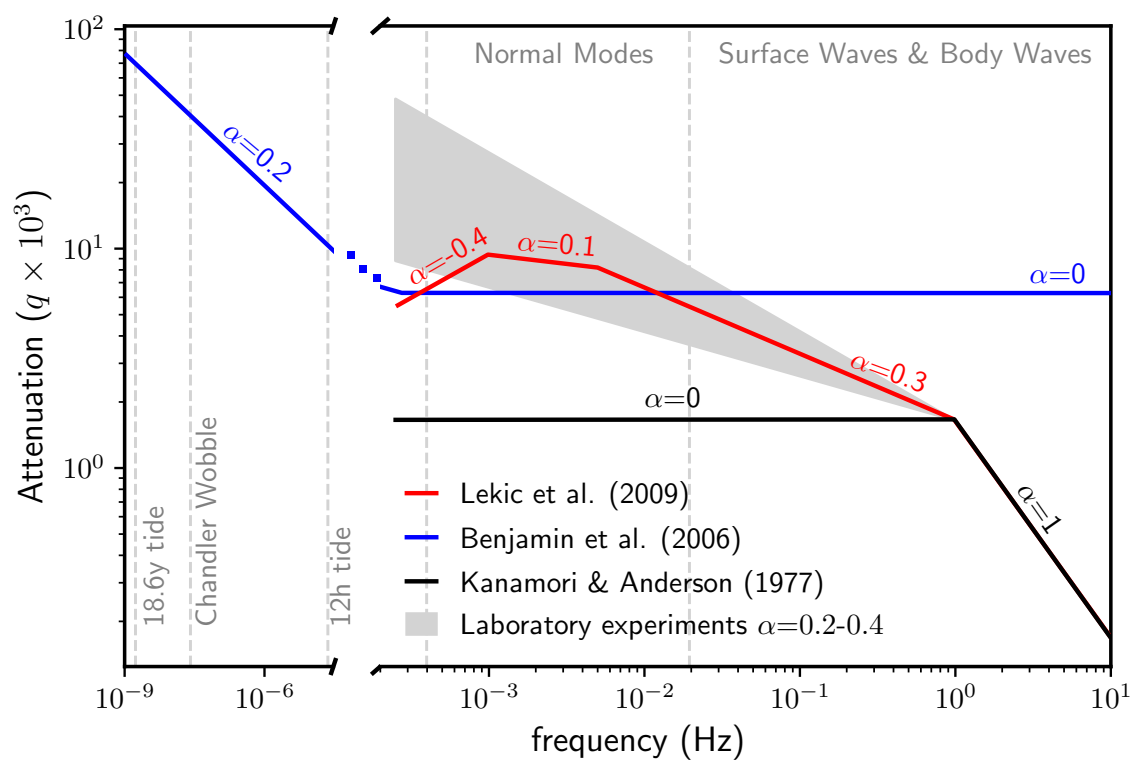

Figure 1.3: Attenuation $\left(q_{\mu} \times 10^{3}\right)$ as a function of frequency using $q \propto \omega^{-\alpha}$, with values for $\alpha$ from Lekić et al. (2009) obtained by using surface waves and normal modes (red), Benjamin et al. (2006) using body tides and the Chandler Wobble (blue), Kanamori and Anderson (1977) using linear viscoelastic models (black), and from various laboratory experiments (grey), e.g. Jackson (2015).

frequency and depth may lead to the radial variations of attenuation being mistakenly interpreted as the behaviour of $\alpha$ as a function of frequency (Lekić et al., 2009).

\subsubsection{Seismic observations}

Above, we have discussed the frequency dependence of seismic attenuation in general terms, but now we turn to the more detailed seismic observations. $1 \mathrm{D}$ and 3D attenuation can be measured using high-frequency body waves, intermediate-frequency surface waves or low-frequency normal modes. Body wave attenuation studies typically measure attenuation in terms of $t^{*}$ (e.g. Hwang and Ritsema, 2011), which is defined as

$$
t^{*}=\int_{\text {ray }} \frac{d s}{Q v}
$$

where the integral is done over the ray path $s$, and $v$ is the wave velocity along the path. If $Q$ is constant, this can be simplified to $t^{*}=\frac{t}{Q}$. Body wave studies usually average or stack their data in order to correct for elastic focussing effects, as well as source and receiver effects (e.g. Lawrence and Wysession, 2006a; Hwang and Ritsema, 2011). Other more recent body waves studies (e.g. Konishi et al., 2020) have used finite-frequency tomography, which is based on the travel-times and amplitudes of nearly vertically traveling $\mathrm{ScS}$ phases. 
Global surface wave attenuation models have been primary built using either Rayleigh wave amplitudes datasets (e.g. Dalton et al., 2008), or by computationally expensive Full-Waveform Inversion (FWI) schemes, which alternate between inverting for elastic and anelastic structure (e.g. Zhu et al., 2013; Karaoğlu and Romanowicz, 2018). Just as with body waves, models based on surface wave amplitude data also need to be corrected for elastic focussing effects, as well as the uncertainties in the moment magnitude of earthquakes and impulse response of stations. This correction can be done by inverting for intrinsic attenuation as well as corrections to the ray path, earthquake moment magnitude and station response (e.g. Bao et al., 2016). FWI studies, on the other hand, do not require focussing effect corrections, but they still need corrections for receiver and source effects in their inversions (e.g. Karaoğlu and Romanowicz, 2018). Although surface waves studies have been able to provide ever more detailed attenuation tomographic models, unlike body waves and normal modes, they are only able to image the upper and mid mantle (up-to $900 \mathrm{~km} \mathrm{depth}$ ).

Normal modes ${ }^{3}$, which are the main focus of this thesis, are sensitive to the whole Earth. Attenuation studies based on normal modes rely on $Q$ value measurements (e.g. Durek and Ekström, 1995; Deuss et al., 2013) and anelastic structure coefficient observations, which have been mainly performed for the inner core (e.g. Mäkinen and Deuss, 2013; Pachhai et al., 2020), with a few studies available for the mantle (e.g. Masters et al., 2000a; Masters et al., 2000b).

In the next section, we will describe particular 1D and 3D $Q$ models and outline what they tell us about the Earth's structure. These attenuation models will be discussed in terms of shear attenuation $q_{\mu}=1 / Q_{\mu}$, which describes the dissipation of shear energy, and bulk attenuation $q_{\kappa}=1 / Q_{\kappa}$, which describes the dissipation of compressional energy.

\subsubsection{1D seismic attenuation models}

Global 1D $Q$ models have been created using either one seismic data type or a combination of different data types across a wider frequency band. Most 1D seismic $Q$ models have been constructed either using (i) low frequency normal modes (e.g. Sailor and Dziewonski, 1978; Durek and Ekström, 1995; Durek and Ekström, 1996; Widmer-Schnidrig et al., 1991), (ii) high-frequency body waves (e.g. Lawrence and Wysession, 2006a; Hwang and Ritsema, 2011), or (iii) a combination of low-frequency normal modes, intermediate-frequency surface and high-frequency body waves (e.g. Anderson and Hart, 1978; Dziewonski and Anderson, 1981; Durek and Ekström, 1995; Masters and Widmer-Schnidrig, 1995; Durek and Ekström, 1996; Romanowicz and Durek, 2000; Resovsky et al., 2005). These models mainly constrain shear wave attenuation $q_{\mu}$, and they all show that shear attenuation $q_{\mu}$ dominates over bulk attenuation $q_{\kappa}$ (see Fig. 1.4). Although bulk attenuation has been considered negligible in some studies, in order to explain certain normal mode observations (i.e. radial modes ${ }^{4}$ ), finite bulk attenuation is needed somewhere in the Earth.

\footnotetext{
${ }^{3}$ Normal modes are long-period oscillations or standing waves along the Earth's radius and surface, that only exist for discrete frequencies. See Chapter 2 for an extensive explanation.

${ }^{4}$ Radial modes are long-period oscillations involving the radial expansion and contraction of the whole Earth. They are dominated by compressional energy, which is dissipated much more slowly than shear energy. See Chapter 4 for an extensive explanation.
} 

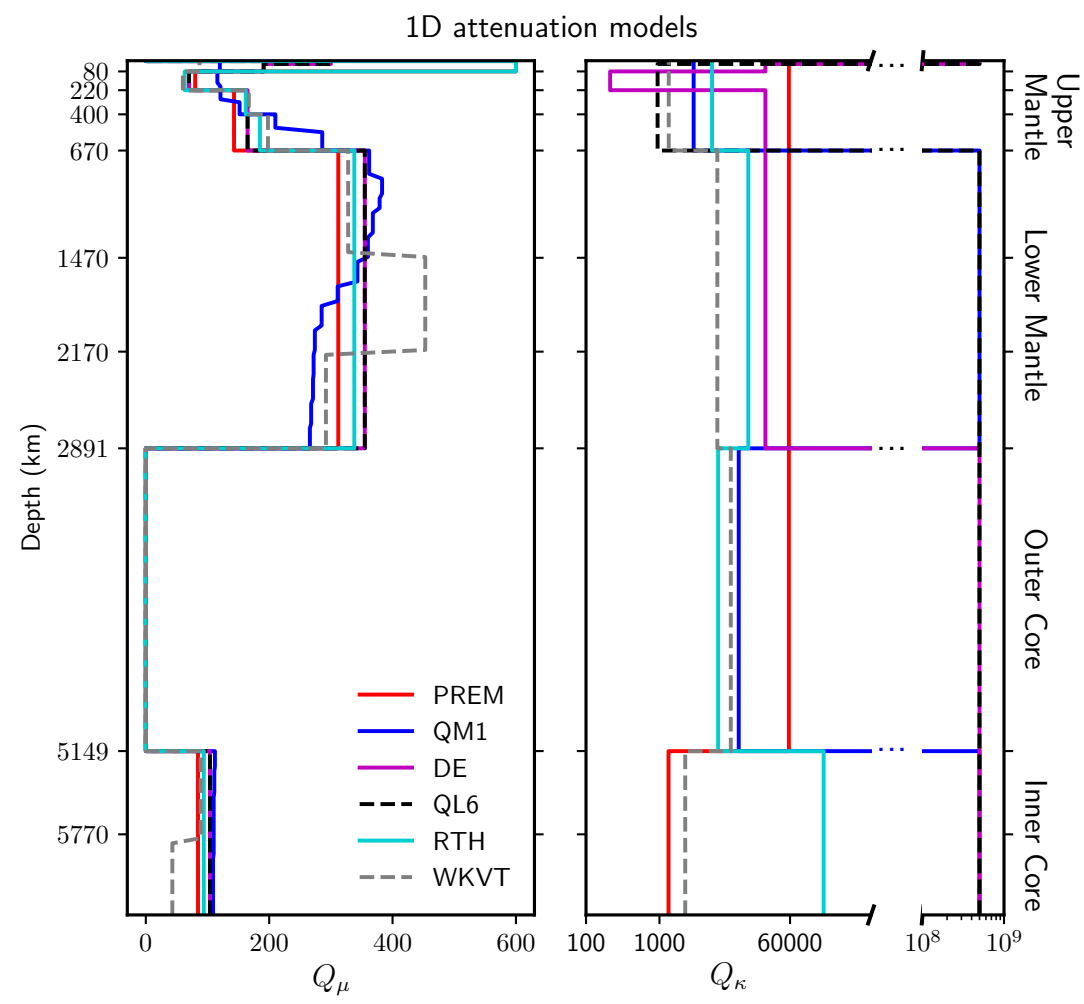

Figure 1.4: $1 \mathrm{D}$ attenuation models based on normal modes, for shear attenuation $Q_{\mu}$ (left) and bulk attenuation $Q_{\kappa}$ (right). Models PREM (Dziewonski and Anderson, 1981); QM1 (Widmer-Schnidrig et al., 1991); DE (Durek and Ekström, 1995); QL6 (Durek and Ekström, 1996); RTH (Resovsky et al., 2005); and WKVT (de Wit et al., 2014).

However, its exact depth location is still a matter of debate, with some studies placing the region of finite $Q_{\kappa}$ in the upper mantle (Sailor and Dziewonski, 1978) or the lithosphere (Durek and Ekström, 1995), while other studies prefer the inner core (Anderson and Hart, 1978; Dziewonski and Anderson, 1981) or even the outer core or lower mantle (Resovsky et al., 2005).

Due to the frequency dependence of $Q$ (see the previous section), 1D $Q$ models based on low frequency normal modes and intermediate frequency surface waves show more agreement between themselves than with high frequency body-wave based $Q$ models. Body waves data have been used to determine average $Q$ values of the mantle and the inner core; however, they usually just provide averages for particular regions of Earth (e.g. ScS seeing particular subduction zones). Normal modes, on the other hand, are able to provide true global $Q$ averages because of their global nature. In this thesis we are mainly concerned with normal modes and because of this, here, we concentrate on $1 \mathrm{D} Q$ models primarily based on normal mode and surface wave data. 
1D shear attenuation models made using normal modes (Fig. 1.4) show a strongly attenuating (i.e. low $Q_{\mu}$ ) upper mantle and inner core; the fluid outer core is not transmitting shear waves leading to $Q_{\mu}=0$. The upper mantle displays a layer with strong attenuation between $80-220 \mathrm{~km}$, which corresponds to the low velocity zone and it is generally related to the boundary between the lithosphere and the asthenosphere. Following this high attenuation region, we see a sharp gradient across the mantle transition zone, where attenuation decreases (i.e. an increase in $Q_{\mu}$ ). After the transition zone $(670 \mathrm{~km})$, the mid-mantle $(670-2000 \mathrm{~km})$ becomes more weakly attenuating (increase in $Q_{\mu}$ ), and it only becomes more strongly attenuating again (decrease in $Q_{\mu}$ ) in the lowermost mantle $(>2000 \mathrm{~km}$ ). Despite this increase in attenuation, the lower mantle is still significantly less attenuating than both the upper mantle and the inner core. Inner core attenuation shows great agreement across all models $\left(Q_{\mu}=90-110\right)$, with only the model from de Wit et al. (2014) showing a difference between the uppermost and innermost inner core. On the other hand, the $1 \mathrm{D} Q_{\kappa}$ bulk attenuation models vary widely in the location of finite bulk attenuation and differ by several orders of magnitude in some parts of the Earth.

It is important to highlight that almost all previous 1D attenuation models have assumed $Q$ to be independent of frequency. This limitation is in strong contrast to one of the most robust aspects of laboratory experiments which find a strong frequency dependence of $Q$ (e.g. Fig. 1.6), and implies a systematic tendency in the 1D $Q$ profiles to overestimate attenuation in the deep Earth (Karato, 2008).

\subsubsection{3D seismic attenuation models}

Now we turn our attention to $3 \mathrm{D}$ seismic attenuation models. Although many regional 3D attenuation studies have been published since the 1960s, here, we will only discuss the general findings of available global attenuation $3 \mathrm{D}$ studies of the mantle and the inner core.

Global 3D attenuation studies of the mantle have mainly concentrated on the upper mantle, and tomographic attenuation models have been available since the mid 1990s. These models have been made using inversion of either surface wave data (Romanowicz, 1995; Selby and Woodhouse, 2002; Gung and Romanowicz, 2004; Dalton et al., 2008; Adenis et al., 2017; Karaoğlu and Romanowicz, 2018) or body wave data (Bhattacharyya et al., 1996; Reid et al., 2001; Warren and Shearer, 2002). Because the 3D intrinsic attenuation needs to be separated from focussing and scattering effects, all these models required approximations to correct for the effect of $3 \mathrm{D}$ velocity variations on wave propagation. The resulting models show their greatest agreement for shallow depths up-to $200 \mathrm{~km}$ (e.g. Fig. 1.5), however even there they still show significant differences especially in regions of low attenuation. In general, in the upper mantle a strong correlation between regions of low velocity and strong attenuation has been found (e.g. the mid-oceanic spreading ridges), and also between regions of high velocity and low attenuation (e.g. continental cratons), which points to upper mantle heterogeneity being driven by temperature variations.

In contrast, observing $3 \mathrm{D}$ intrinsic attenuation variations in the lower mantle has presented more severe difficulties both due to the weaker dissipating behaviour of the mantle beyond the transition zone, and also due to the strong trade-off between 

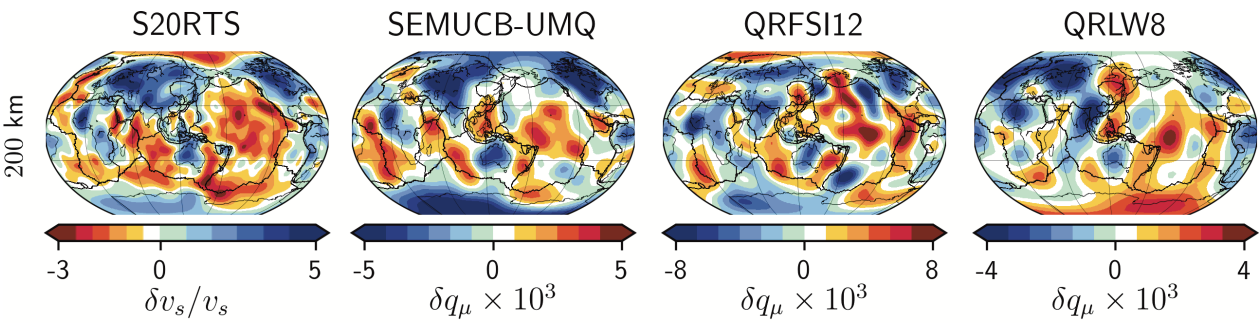

Figure 1.5: Comparison between shear velocity model S20RTS (Ritsema et al., 1999) and three global shear attenuation models: SEMUCB-UMQ (Karaoğlu and Romanowicz, 2018), QRFSI12 (Dalton et al., 2008) and QRLW8 (Gung and Romanowicz, 2004). All attenuation models are plotted in terms of $\delta q_{\mu} \times 10^{3}$ at $200 \mathrm{~km}$ depth. $\delta q_{\mu} \times 10^{3}$ is presented as perturbation value and in percentage. Tectonic plate boundaries (Bird, 2003) are included for comparison with the $3 \mathrm{D}$ global shear attenuation models.

frequency and depth dependence, which becomes more important the deeper in the Earth we try to observe attenuation variations. Surface waves, which have been largely used to measure 3D attenuation in the upper mantle, do not penetrate beyond the transition zone, while body waves need extensive approximations to correct for focussing and scattering when measuring attenuation. Only two body wave studies have managed to make observations of regional lateral variations in attenuation in the lower mantle, however without taking focussing and scattering into account (Lawrence and Wysession, 2006b; Hwang and Ritsema, 2011), and thus far no lower mantle global attenuation models have been published. Lawrence and Wysession (2006b) identified a region in northeast Asia at the top of the lower mantle with anomalously strong attenuation and slight decrease in velocity, and interpreted it as a subducting slab transporting water into the lower mantle; while Hwang and Ritsema (2011) showed that the Pacific LLSVP was on average more strongly attenuating than its surrounding mantle for depths up-to $2470 \mathrm{~km}$.

Even less is known about 3D attenuation variations in the inner core. 3D velocity studies of the inner core have found cylindrical anisotropy since the mid 1980s, with its fast velocity direction aligned with the Earth's rotation axis (e.g. Woodhouse et al., 1986; Morelli et al., 1986). More recent studies of 3D inner core attenuation have also found attenuation anisotropy(Souriau and Romanowicz, 1996; Souriau and Romanowicz, 1997), with the fast velocity direction being the most strongly attenuating (e.g. Masters et al., 2000a; Masters et al., 2000b; Mäkinen and Deuss, 2013; Pachhai et al., 2020). This observation is opposite to what is seen in the Earth's upper mantle, where the slow velocity is the most strongly attenuating and therefore, requires a different explanation. Short period body wave observations might be explained by a scattering mechanism due to alignment of anisotropic crystals within the inner core or by the presence of liquid inclusions (Singh et al., 2000), but the observation of attenuation anisotropy in long-period normal modes requires an alternative explanation (Mäkinen et al., 2014). 


\subsubsection{Mineral physics}

In order to interpret the observed $1 \mathrm{D}$ and $3 \mathrm{D}$ seismic attenuation models in terms of variations in temperature, melt or composition, we need mineral physics. Microscopic relaxation mechanisms have been studied using rock experiments in the laboratory. These experiments have provided constraints on the main physical mechanisms controlling attenuation, such as grain boundary sliding or dislocation processes, as well as their corresponding frequency dependence and the overall pressure and temperature dependence of attenuation (i.e. Faul and Jackson, 2015).

Attenuation depends exponentially on temperature. From experimental results, this relationship can be expressed as (e.g. Karato, 1993; Jackson, 2015)

$$
q=A \omega^{-\alpha} e^{-\alpha H / R T}
$$

where $R$ the gas constant, $T$ is temperature and $H=E+P V$ is the activation enthalpy, which depends on the internal activation energy $E$, pressure $P$ and activation volume $V$. Unfortunately, activation volume might be difficult to constrain, because of the limited pressure ranges available in most laboratory experiments (Jackson et al., 2000). For this reason, Eq. 1.3 can be re-parametrised as (e.g. Karato and Jung, 1998; Jackson, 2015)

$$
q=A \omega^{-\alpha} e^{-\alpha g T_{m} / T}
$$

where $T_{m}$ is the melting temperature, $T_{m} / T$ is the homologous temperature, and $g$ is a dimensionless constant, which itself depends on $H, T_{m}$ and $R$. Eq. 1.4 substitutes the $V$ dependence with $T_{m}$, which is easier to measure in laboratory experiments. Furthermore, this $T_{m}$ dependence allows grain size and frequency to be related by a simple scaling law (Sato et al., 1989).

Unlike attenuation, shear velocity, which depends on the shear modulus $\mu$, does not have an exponential dependence on temperature (see Fig. 1.6, reproduced from Faul and Jackson, 2005). However, the shear modulus still depends non-linearly on temperature, with this non-linearity increasing with increasing temperature and period, and decreasing with grain size (Figs. 1.6a-1.6b). This means that at higher temperatures, a small change in temperature is able to produce significant velocity changes, in contrast to at lower temperatures which require larger temperature variations to give the same velocity change (Fig. 1.6a).

\subsubsection{Mantle}

Torsional forced oscillation experiments on olivine, which is the dominant mineral in the upper mantle, show that increasing temperature lowers the shear modulus, while it increases attenuation (see Fig. 1.6, Gribb and Cooper, 1998; Jackson and Faul, 2010a). The process responsible for this is boundary sliding, which strongly depends on the applied frequency in the torsional experiments and the grain size of the olivine sample. Extrapolations of the experimental data are needed, given that laboratory experiments use much smaller grain sizes than the grain size present in the mantle (Faul and Jackson, 2005). 


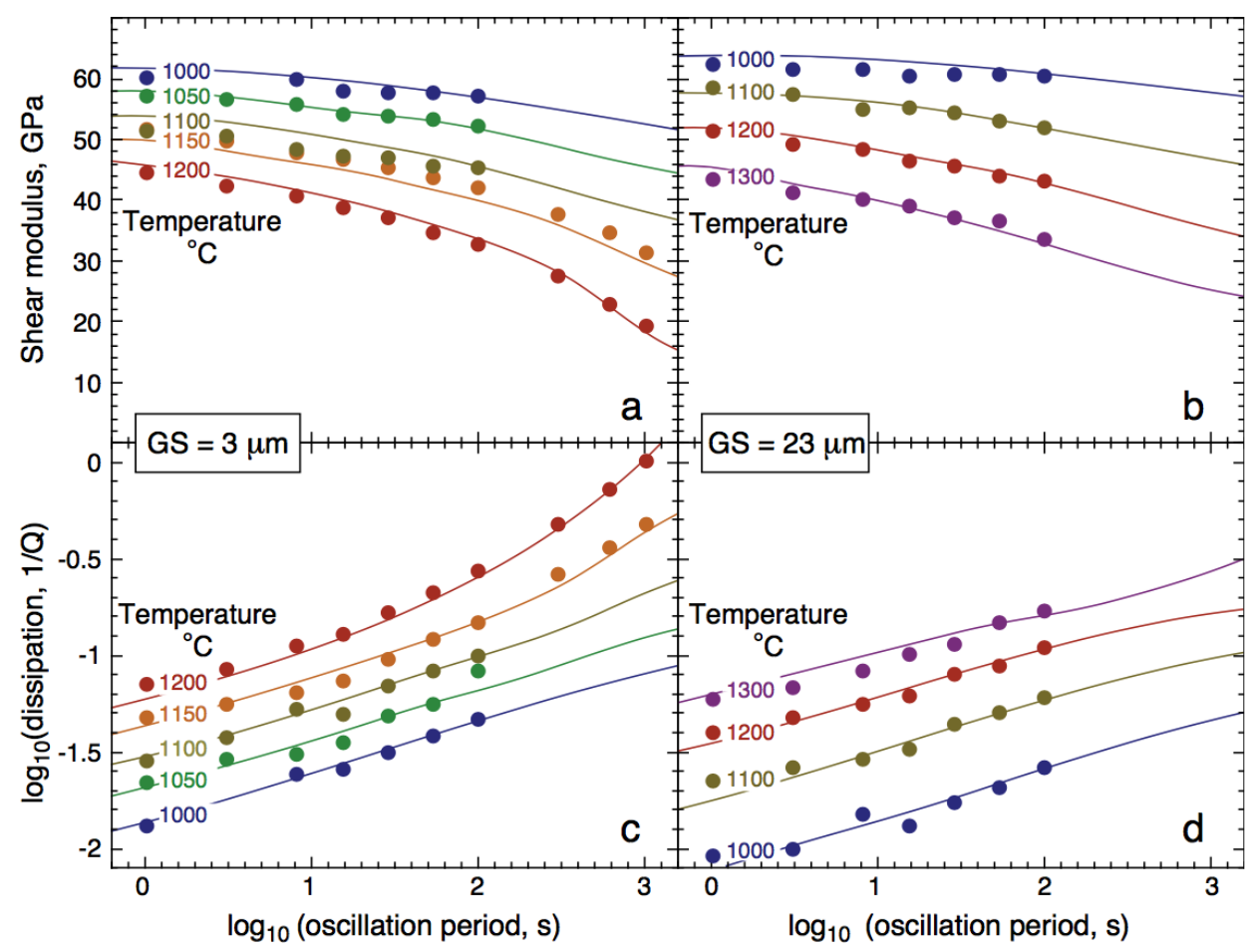

Figure 1.6: Experimental results for torsional experiments on olivine, reproduced from Faul and Jackson (2005), showing (a, b) shear modulus (GPa) and (c, d) attenuation $(1 / Q)$ as a function of oscillation period and temperature for two different grain sizes of $3 \mu \mathrm{m}$ and $23 \mu \mathrm{m}$. The circles represent the data from the experiments at different temperatures and periods, and the solid lines the fit of the extended Burgers model.

The presence of water or partial melt, or a change in composition, also have their distinct effects on attenuation and the shear modulus. Mineral compositional changes have negligible influence on attenuation, while they can greatly alter the shear modulus (Lee, 2003). On the other hand, inclusions of water in minerals increase attenuation, while mildly affecting velocity through dispersion (Karato and Jung, 1998; Karato, 2003; Aizawa et al., 2008). The influence of partial melt depends on its mechanism. Partial melt presence enhanced by grain boundary sliding increases attenuation and decreases velocity (Jackson et al., 2004; Faul et al., 2004), whereas a melt squirt mechanism only affects the shear modulus and not attenuation (Hammond and Humphreys, 2000a; Hammond and Humphreys, 2000b).

From the limited studies of the anelastic behaviour of lower mantle minerals, we know that both analogue titanate perovskites (Webb et al., 1999) and $\mathrm{MgO}$ (Webb and Jackson, 2003; Barnhoorn et al., 2016) possess similar anelastic properties as olivine. This means that increasing temperature in these lower mantle minerals also lowers the shear modulus and increases attenuation. The dissipating behaviour of 
the newly discovered post-perovskite phase (Murakami et al., 2004; Oganov and Ono, 2004; Tsuchiya et al., 2004) has not been tested in laboratory experiments yet. However, from recently available, ab-initio molecular simulations we know that post-perovskite is potentially a highly attenuating mineral (Goryaeva et al., 2016).

\subsubsection{Inner core}

The crystalline structure of the inner core has been studied using both diamond anvil cell experiments (e.g. Tateno et al., 2010; Tateno et al., 2012; Sakai et al., 2011) and ab-initio molecular simulations (e.g. Vočadlo et al., 2003; Vočadlo, 2007). From these studies, it has been widely accepted that the most stable phase of pure iron is hcp (hexagonal close-packed), which might also remain stable even after accommodating a few percent of light elements or alloying iron with nickel and/or silicon. However, the problem is that the shear wave velocity as measured by seismic normal modes is much smaller than the shear wave velocity obtained for iron at inner core pressure and temperature (Deuss, 2008). Furthermore, its strongly attenuating behaviour seen in seismic studies, has been even more difficult to explain for an inner core made of mostly pure iron, with partial melting first being proposed to explain the anelastic behaviour (e.g. Cormier, 1981).

An alternative explanation comes from the torsional forced oscillation experiments of Jackson et al. (2000) for iron at increasing temperature, which showed that the inner core may be strongly attenuating in shear without the need for partial melt. Using the same idea applied for the mantle mineral olivine, Jackson et al. (2000) found that when you increase $T$ in the bcc (body-centered cubic) and fcc (facecentered cubic) iron phases, the shear modulus lowers non-linearly. This effect becomes stronger the closer you reach the melting temperature $T_{m}$; a homologous temperature of $T_{m} / T \sim 1$ is expected in the inner core, thus very close to the melting temperature. Jackson et al. (2000) found that there is no actual melting, but that the mineral softens the closer you reach the melt temperature, lowering the shear modulus and also increasing attenuation. This is a principle that is well studied in metals, and we already discussed above that it is also applicable for mantle minerals olivine and $\mathrm{MgO}$. In Jackson et al. (2000) the anelastic effect was visible, but not strong enough to lower $v_{s}$ of iron to seismic values. Using ab-initio simulations, the recent work by Martorell et al. (2013) showed that the effect becomes very strong in the hcp (hexagonal-close packed) phase just before melting (hence pre-melting) and that it does lower $v_{s}$ to seismically observed values.

Another recent study using ab-initio molecular simulations proposed that the unique low viscosity of the bcc structure (Belonoshko et al., 2019), can also explain the strong attenuation of the inner core. This explanation depends on bcc actually being the stable phase of iron at inner core pressures, because only hcp is found in diamond anvil experiments (e.g. Tateno et al., 2010). Belonoshko et al., 2017 has recently shown in ab-initio molecular simulations that bcc may be stable at inner core conditions and some evidence was also seen in high pressure laboratory experiments (Hrubiak et al., 2018). If indeed stable at inner core pressure and temperature, then the highly attenuating bcc phase would also point to a lower shear velocity than previous studies (Belonoshko et al., 2007). This means that both the hcp and bcc 
iron phases would be able to explain the anelastic behaviour of the inner core seen in seismic observations.

\subsubsection{Geodynamics}

Up-to this point, we have discussed attenuation in terms of seismic measurements and mineral physics, however geodynamical models based on real observations also need to be considered. Here, we will briefly discuss how they relate to mantle and inner core structure and how our understanding of seismic attenuation influences geodynamical models.

$3 \mathrm{D}$ density variations in the mantle have been studied in seismology and are essential in mantle convection modelling because they are driving the flow. However, density, just like attenuation, has been notoriously difficult to constrain using seismic data. The problem is that body waves are not sensitive to density variations, and that only normal modes (and surface waves to a much smaller extent) are able to provide information on density.

The thermal or compositional origin of the Large Low Shear Velocity Provinces (LLSVPs) at the base of the lower mantle under Africa and the Pacific (Fig. 1.7) has long been debated and it is thought that we can constrain their origin if we would know their density. This is because, if the LLSVPs have a lower density in combination with lower shear wave velocity, then they will be thermal anomalies which would be transient and possibly short-lived (Zhang et al., 2010). However, if the LLSVPs are denser than the surrounding mantle, then they will require compositional heterogeneity due to, for example, enrichment in iron which could make them long-lived, potentially forming stable 'anchors' at the base of the mantle that have a unique position with respect to the Earth's moment of inertia (Dziewonski et al., 2010), and have been influencing the pattern of mantle convection for a long time (Torsvik et al., 2014).

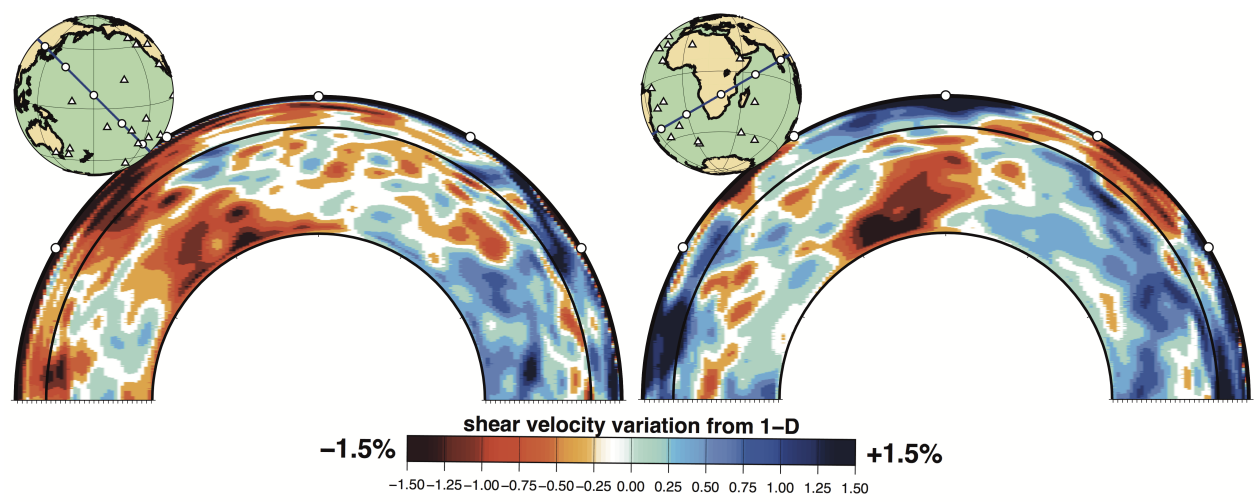

Figure 1.7: Cross-sections of shear velocity tomographic model S40RTS (Ritsema et al., 2011), showing the Large Low Shear Velocity Provinces (LLSVPs) under the Pacific (left) and Africa (right). 
Past studies have mainly used normal modes (Ishii and Tromp, 1999; Trampert et al., 2004; Koelemeijer et al., 2017), and one recent study also used tidal waves (Lau et al., 2017), to image the density of the LLSVPs and have reported sometimes seemingly conflicting results. Most studies find denser LLSVPs (Ishii and Tromp, 1999; Trampert et al., 2004; Lau et al., 2017), pointing to a compositional origin and a likely enrichment in iron. However, one study using Stoneley modes found neutrally buoyant or even lighter LLSVPs (Koelemeijer et al., 2017), pointing to a thermal origin. Some studies even report difficulties in robustly constraining density using inversion methods based on normal mode splitting functions (Masters et al., 2000c; Romanowicz, 2001; Kuo and Romanowicz, 2002; Akbarashrafi et al., 2017; Akbarashrafi, 2020). Here, we strive to add additional constraints for lower mantle structure by studying lateral variations in attenuation in comparison to velocity. One of the main challenges of this thesis is to attempt to measure $3 \mathrm{D}$ variations in attenuation for the lower mantle and use these to answer the question of the thermal or compositional origin of the LLSVPs.

Again, even less is known about the dynamics of the inner core, and what might cause the observed cylindrical anisotropy in both velocity and attenuation. Two main models have been proposed that may generate crystal structure alignment and that are able to reproduce cylindrical anisotropy (e.g. Deuss, 2014): (i) solidification texturing (e.g. Karato, 1993), which predicts an alignment with the magnetic field or the outer core flow and (ii) texturing being due to deformation after solidification (e.g. Yoshida et al., 1996). Three different mechanisms have been proposed for deformation texturing: (a) thermal convection (Jeanloz and Wenk, 1988; Romanowicz et al., 1996), with convection at the early stages of the inner core history potentially explaining its layered structure (Cottaar and Buffett, 2012); (b) deformation by the Maxwell stress of the magnetic field (Karato, 1999; Buffett and Wenk, 2001); and (c) predominantly inner core equator growth leading to a flow from the equator to the poles and so aligning the iron crystals (Yoshida et al., 1996). Currently there is no consensus on a particular geodynamical model to explain the inner core anisotropy, let alone its attenuation anisotropy.

\subsection{Motivation}

The purpose of this thesis is to tackle unresolved questions regarding the attenuation structure of the Earth's mantle and inner core. Over the last few decades our knowledge of Earth's attenuation variations has lagged behind the advancements made by tomographic velocity models, when ever more accurate models with higher resolution are now available. Here, we attempt to bridge that knowledge gap by using whole Earth oscillations or normal modes, which allow us to study both lateral and depth variations of velocity and attenuation simultaneously. At the low frequencies of the normal modes, scattering, which acts as apparent attenuation, becomes less important due to the longer wavelength of the seismic waves when compared to the small size of the scatterers. In addition, within the framework of first order perturbation theory, it is relatively straightforward to include attenuation when using normal modes by mathematically changing the elastic parameters (i.e. shear modulus $\mu$ and bulk modulus $\kappa$ ) 
from real to complex numbers, where the real part describes the elastic parameters and the imaginary part contains the anelastic parameters in terms of attenuation.

Seismic attenuation observations complement the knowledge acquired from seismic velocity observations and experimental studies on the elastic and anelastic behaviour of mantle and inner core minerals. For example, 3D attenuation may allow us to constrain the thermal or compositional origin and geodynamic mechanisms driving distinct features seen in the Earth's mantle, such as the Large Low Shear Velocity Provinces (LLSVPs) in the lower mantle underneath Africa and the Pacific (Fig. 1.7). Attenuation, because of its exponential dependence on temperature, is especially suitable to identify high temperature anomalies, allowing us to distinguish between thermal and compositional heterogeneities in the Earth's upper and lower mantle and aid in interpreting seismic observations in terms of mantle convection.

\subsection{Outline}

In this thesis we use whole Earth oscillations, or normal modes, to measure variations in attenuation in the Earth's mantle and inner core. To do this we use splitting functions, which for a given mode show regional variations in the frequency of the mode and are depth weighted averages of how a particular mode 'sees' the Earth. Splitting functions are linearly dependent on heterogeneous and anisotropic structure, and depend on both elastic (i.e. density and velocity) and anelastic (i.e. attenuation) structure. They can be included as data in making seismic tomography models, and we use them to study 1D and 3D anelastic structure in the mantle and inner core.

We first give a general description of normal mode theory and our method in Chapter 2. We describe how elastic and anelastic splitting functions are measured from seismic spectra of large earthquakes, using the iterative spectral fitting method and a least-squares inversion scheme. At the end of this chapter, we also describe the data we use, and list our earthquake catalogue comprised of 104 events, which we will use to perform our measurements in the succeeding chapters.

We then proceed by studying attenuation in the inner core. We start in Chapter 3 by assessing the effects of attenuation in the inner core by measuring cross-coupling, or resonance, for 1D $Q$ structure between pairs of inner core sensitive modes that are very close in frequency. We mainly focus on the heavily debated mode pair of ${ }_{10} S_{2-11} S_{2}$, which cross-couples strongly for $1 \mathrm{D}$ structure and is sensitive to shear wave velocity in the inner core. The oscillation characteristics and $Q$ values of modes ${ }_{10} S_{2}$ and ${ }_{11} S_{2}$ change non-linearly under a small $1 \mathrm{D}$ perturbation to the inner core velocity model. In the past, this has led to the measurements of mode ${ }_{10} S_{2}$ to be questioned, as its large spectral amplitude in earthquake data and its small amplitude in synthetic data implied a weakly attenuating inner core, which contradicted other normal mode and body wave observations. As a consequence, modes ${ }_{10} S_{2}$ and ${ }_{11} S_{2}$ have commonly been excluded from normal mode studies of the inner core. Here, we include the non-linear $1 \mathrm{D}$ perturbation effects by allowing the mode pair to cross-couple through 1D structure and treat both modes as one hybrid mode. From our measurements, we find that either an increase in inner core shear velocity of $0.5 \%$ or a decrease in inner core radius of $0.5 \%$ explains the observation of modes ${ }_{10} S_{2^{-} 11} S_{2}$, without requiring a weakly attenuating inner core. Thus, mode ${ }_{10} S_{2}$ is 
now in agreement with other normal mode and body wave observations and also favours a strongly attenuating inner core.

We then move on to measuring radial modes in Chapter 4, which depend strongly on compressional energy and are sensitive to the whole Earth. We use the radial modes to provide constraints on $1 \mathrm{D}$ bulk attenuation in the mantle and core which is important before turning our attention later in the thesis to $3 \mathrm{D}$ attenuation variations in the mantle. The presence of significant 1D bulk attenuation somewhere in the Earth had been inferred from the attenuation of radial modes in previous studies. It is often assumed that radial modes do not cross-couple to other modes; when they are measured in isolation, they only depend on 1D structure and are constrained by their center frequencies and $Q$ values. We investigate the importance of cross-coupling for radial modes by measuring them both in self-coupling (i.e. isolated from other modes), and in cross-coupling (i.e. resonating) with their closest $l=2$ modes. By allowing them to resonate, the radial modes become sensitive to $3 \mathrm{D}$ structure. We measured 11 radial modes (i.e. $n=0,1,2, \ldots, 9,11$ ), and find that by accounting for degree-two structure, their measured center frequency shifts become systematically smaller with respect to PREM. Our $Q$ value measurement of the gravest radial mode ${ }_{0} S_{0}$ indicates that less bulk attenuation might be required somewhere in the Earth than previously thought. In addition, the smaller center frequency shifts observed with cross-coupling, agree with lower values of radial anisotropy in the uppermost inner core, than the ones inferred from just measuring the modes in self-coupling. Furthermore, measuring the cross-coupling structure between radial modes and their closest $l=2$ modes also provides us with new constraints on inner core cylindrical anisotropy structure.

Finally, we turn our attention to $3 \mathrm{D}$ variations in mantle attenuation. The signal of $3 \mathrm{D}$ attenuation in normal mode spectra might be small, so in Chapter 5 , we first test our ability to observe 3D mantle attenuation in synthetic normal mode spectra and using these spectra to measure anelastic splitting functions. We show that synthetic spectra for modes of the $1^{\text {st }}$ and $2^{\text {nd }}$ overtone branches are most strongly affected when $3 \mathrm{D}$ variations in attenuation are included. Fundamental modes $(n=0)$ show less sensitivity to 3D variations in attenuation, however this is dependent on the depth of the earthquakes. We also test the potential trade-offs with the earthquake moment magnitude when measuring anelastic splitting functions which are sensitive to attenuation. We find that the effect of earthquake magnitude might be a problem only when the attenuation signal of a given lower mantle mode is less than $50 \%$ smaller than the scaled amplitude signal from existing upper mantle tomographic attenuation models made using surface waves. Based on our test results, we regard our method of measuring anelastic splitting functions sufficiently robust to measure $3 \mathrm{D}$ variations in mantle attenuation, and we believe that our observations are well constrained for both the upper and lower mantle.

Once we are satisfied that the signal of $3 \mathrm{D}$ attenuation might be robustly retrieved from normal mode spectra, we apply our methods to real earthquake data in Chapter 6 . We measure elastic and anelastic structure simultaneously for fundamental modes and the $1^{\text {st }}$ and $2^{\text {nd }}$ overtones, which are sensitive to the upper and lower mantle. Measuring the elastic and anelastic splitting functions simultaneously, allows us to 
separate trade-offs with elastic effects, such as focussing due to large scale velocity structure, from intrinsic attenuation. Just as in previous studies using surface waves and long-period waveforms, we find that in the upper mantle low velocity regions are correlated with strongly attenuating regions. Comparing these observations with experimental data for olivine shows that the upper mantle observations are dominated by lateral temperature variations, in agreement with previous studies. In the lower mantle, we find that the strongest attenuation is found in two high velocity regions centered around Oceania and central America, and not in the LLSVPs. The strong attenuation found in the high velocity regions surrounding the LLSVPs is opposite of what we see in the upper mantle, and we interpret them as potentially due to small grain-sizes in combination with cold temperatures, and/or the presence of post-perovskite, which might be strongly attenuating. The weakest attenuation is found in low velocity regions in the northern part of the Pacific LLSVP near Hawaii, and in the southern part of the African LLSVP. This indicates that these regions might be dominated by compositional variations rather than temperature. Such compositional variations could potentially be due to iron enrichment, which agrees with larger density found in the same regions. In the central part of the LLSVPs we find average attenuation, which could be due to large grain-sizes and hot temperatures in perovskite, in combination with post-perovskite, which might overwhelm the effect of perovskite on the LLSVPs.

At the end of this thesis, we finish with a synthesis of our measurements and findings in Chapter 7, where we put our results into the further context of current and future research. 



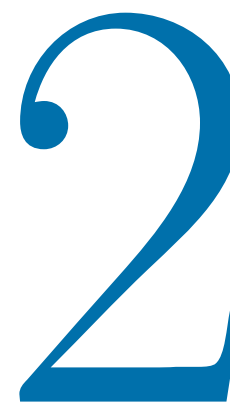

\section{Method and Data}

The objective of this chapter is to give a general background of the method we follow throughout this thesis in measuring splitting functions using normal mode data. However, it is far beyond the scope of this thesis to provide a thorough explanation of the theoretical basis for normal mode seismology (see Dahlen and Tromp, 1998, for a comprehensive overview). First, we give a broad description of normal modes and splitting functions, and we also explain how synthetic seismograms are computed. We define the elastic splitting function, depending on velocity and density heterogeneity, and the anelastic splitting function, depending on attenuation heterogeneity. Then, we describe the inversion method we follow in order to measure elastic and anelastic splitting functions from normal mode spectra. Finally, we outline the seismic data we use to obtain the normal mode spectra required for our splitting function measurements, how it is pre-processed, and we list our full earthquake catalogue.

The content of this chapter was partially extracted from the theory and method sections of the published articles: S. Talavera-Soza and A. Deuss (2020). Constraining 1-D inner core attenuation through measurements of strongly coupled normal mode pairs. Geophysical Journal International 223.1, pp. 612-621 and S. Talavera-Soza and A. Deuss (2021). New measurements of long period radial modes using large earthquakes. Geophysical Journal International 224.2, pp. 1211-1224 


\subsection{Normal modes and splitting functions}

\subsubsection{Normal mode definition}

Normal modes are whole Earth oscillations, which are standing waves along the radius and surface of our planet and as a consequence they only exist for discrete frequencies. There are two types of normal modes (Fig. 2.1): (i) spheroidal modes ${ }_{n} S_{l}$, which consist of $P-S V$ motion and are similar to Rayleigh surface waves, and (ii) toroidal modes ${ }_{n} T_{l}$, which consist of $S H$ motion and are similar to Love surface waves. In a spherically symmetric Earth model, the motion of spheroidal modes is described by the two radial eigenfunctions ${ }_{n} U_{l}$ and ${ }_{n} V_{l}$, which are related to vertical and horizontal motions; toroidal modes are described by one radial eigenfunction ${ }_{n} W_{l}$, which is related to shear motions. Both mode types are characterised by two numbers, the angular order $l$ and the overtone number $n$; we use $k$ to denote a mode with overtone number $n$ and angular order $l$. The overtone number $n$ is related to the radial pattern, and the angular order $l$ to the number of nodal lines over the Earth's surface. Modes with $n=0$ are called fundamentals, and modes with $n>0$ are called overtones.

Each normal mode with a given $l$ forms a multiplet consisting of $2 l+1$ singlets. These singlets are labelled using the azimuthal order $m$, where $-l \leq m \leq l$. In a spherical, non-rotating, elastic, isotropic (SNREI) Earth the $2 l+1$ singlets are degenerate, meaning they all have the same frequency. This degeneracy is removed by the effects of rotation, ellipticity, anisotropy and lateral heterogeneities, which we call splitting, and results in all singlets having a different frequency. We calculate splitting through first order perturbation theory (Dahlen, 1968; Woodhouse and Dahlen, 1978; Woodhouse, 1980), starting from the eigenfrequencies and eigenfunctions of the SNREI Earth model. This calculation can either be done using (i) the self-coupling (SC) approximation, where modes are treated as isolated; (ii) the group-coupling (GC) approximation, which includes the cross-coupling between two or more modes close in frequency; or (iii) full-coupling (FC), were cross-coupling between all modes in a certain frequency band is included (e.g. Deuss and Woodhouse, 2001; Jagt and Deuss, 2021).

a) ${ }_{n} S_{l}$

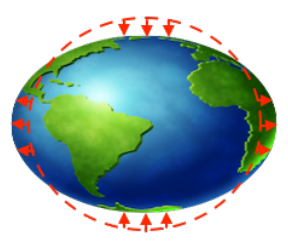

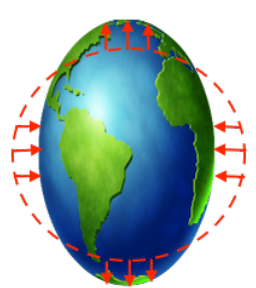

b) ${ }_{n} T_{l}$

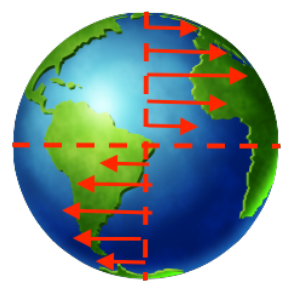

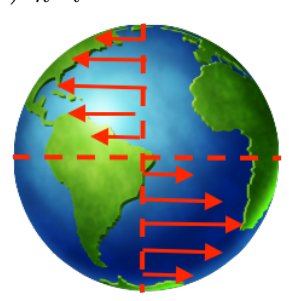

Figure 2.1: Type of modes: (a) spheroidal modes ${ }_{n} S_{l}$, which consist of $P-S V$ motion and are similar to Rayleigh surface waves, and (b) toroidal modes ${ }_{n} T_{l}$, which consist of $S H$ motion and are similar to Love surface waves. 
$\mathrm{SC}$ can be used in cases where modes are sufficiently distant in frequency from their closest neighbours. Thus, the potential cross-coupling with other modes can be considered a second order effect. In SC, modes accumulate information about the Earth's structure in a symmetric manner, and as a result become sensitive only to even-degree structure (e.g. Laske and Widmer-Schnidrig, 2007). When different modes are fairly close in frequency and have similar depth sensitivities, they form 'super-multiplets', and require GC to account for the exchange of energy between modes. Additionally, by allowing mode pairs to cross-couple, odd-degree structure sensitivity may be obtained. In GC, the cross-coupling between different 'supermultiplets' is again considered a second order effect. Although FC provides a more exact theory, it is computationally more expensive and out of the scope of this thesis. Here, we perform measurements in SC and GC, and consider FC a second order effect.

\section{Mode coupling}

Normal modes are mathematically described using spherical harmonics with angular order $l$ and azimuthal order $m$. We also parameterise the structure in the Earth in terms of spherical harmonics, using $s$ to denote the angular order and $t$ to denote the azimuthal order of the structure, which greatly simplifies the mathematics involved.

Mode coupling (i.e. self- or cross-coupling) removes degeneracy from the singlets of a mode, the extent of which depends on the angular order and overtone number of the pair of modes, the angular order of the 3D Earth structure and the depth sensitivity of the modes. The process is non-linear and particular to different causes of coupling, e.g. rotation, ellipticity, Coriolis force, etc. This means the behaviour of different types of coupling cannot be generalised to be the same across all coupling normal modes pairs. The coupling rules (e.g. Laske and Widmer-Schnidrig, 2007) for the different kinds of self- and cross-coupling are:

(i) Rotation cross-coupling by the Coriolis force, which occurs between toroidal and spheroidal mode pairs of the type ${ }_{n} S_{l^{-} n^{\prime}} T_{l \pm 1}$, as well as spheroidal mode pairs ${ }_{n} S_{l^{-} n^{\prime}} S_{l}$. There is no Coriolis cross-coupling between toroidal mode pairs;

(ii) Ellipticity cross-coupling, which also occurs between toroidal and spheroidal mode pairs of the type ${ }_{n} S_{l^{-} n^{\prime}} T_{l \pm 1}$, as well as same mode type (toroidal or spheroidal) pairs with $\left|l-l^{\prime}\right|=0$ or $\left|l-l^{\prime}\right|=2$;

(iii) Rotation cross-coupling (e.g. the centrifugal force), which occurs between spheroidal mode pairs of the type ${ }_{n} S_{l^{-} n^{\prime}} S_{l}$, so mode pairs with the same angular order, i.e. $\left|l-l^{\prime}\right|=0$;

(iv) Attenuation cross-coupling, which occurs between spheroidal mode pairs of the type ${ }_{n} S_{l^{-} n^{\prime}} S_{l}$. This coupling is particularly strong when $n^{\prime}=n \pm 1$ (Andrews et al., 2006), so mode pairs with the same angular degree, i.e. $\left|l-l^{\prime}\right|=0$, and differing by one overtone number. See Chapter 3 for an extensive explanation.

(v) In general, two spheroidal or toroidal modes with angular orders $l$ and $l^{\prime}$ are sensitive to structure with angular order $s$ for which $\left|l-l^{\prime}\right| \leq s \leq\left|l+l^{\prime}\right|$. In selfcoupling, a mode is treated as isolated and only couples with itself, hence $k=k^{\prime}$ and a self-coupled mode is sensitive to even structure of degree $s=0,2, \ldots, 2 l$. 
In cross-coupling, two spheroidal or toroidal modes with angular orders $l$ and $l^{\prime}$ are sensitive to $\left|l-l^{\prime}\right| \leq s \leq\left|l+l^{\prime}\right|$ structure. Depending on $\left|l-l^{\prime}\right|$ being even or odd, the cross-coupled pair is sensitive to either only even or only odd-degree structure. See below for a summary:

(a) Self-coupling $\left(k=k^{\prime}\right)$ for either a spheroidal ${ }_{n} S_{l}$ or a toroidal mode ${ }_{n} T_{l}$ $l-l^{\prime}=0$ is even $\rightarrow$ even degree structure sensitivity

(b) Cross-coupling $\left(k \neq k^{\prime}\right)$ between a pair of modes of the same type ${ }_{n} S_{l^{-} n^{\prime}} S_{l^{\prime}}$ or ${ }_{n} T_{l^{-} n^{\prime}} T_{l^{\prime}}$

if $l-l^{\prime}$ is odd $\rightarrow$ odd degree structure sensitivity if $l-l^{\prime}$ is even $\rightarrow$ even degree structure sensitivity

(c) Cross-coupling $\left(k \neq k^{\prime}\right)$ between a spheroidal ${ }_{n} S_{l}$ and a toroidal mode ${ }_{n^{\prime}} T_{l^{\prime}}$ if $\left|l-l^{\prime}+1\right| \leq s \leq\left|l+l^{\prime}+1\right|$ is even $\rightarrow$ odd degree structure sensitivity if $\left|l-l^{\prime}+1\right| \leq s \leq\left|l+l^{\prime}+1\right|$ is odd $\rightarrow$ even degree structure sensitivity In this thesis, we will only focus on cross-coupling between spheroidal modes.

\subsubsection{Splitting functions}

We will use the generalised splitting function approach of Resovsky and Ritzwoller (1998) to measure normal mode splitting both in SC and GC, following the method explained and applied in Deuss et al. (2013). Splitting functions are depth-averaged models of how one particular mode 'sees' the Earth and where locally the frequency of that mode is slightly higher or lower than its center frequency (Woodhouse and Giardini, 1985). They are linearly dependent on heterogeneous velocity, density, attenuation and anisotropic structure, and for this reason can be easily incorporated by other scientists in tomographic modelling inversions (e.g. Ritsema et al., 1999; Ritsema et al., 2011; Moulik and Ekström, 2014; Koelemeijer et al., 2016). Splitting function coefficients are used to calculate the splitting matrix, which together with the effects of the 1D reference model (Dziewonski and Anderson, 1981), ellipticity and rotation allow us to calculate synthetic seismograms. These synthetic seismograms are then compared to earthquake data in the splitting function measurement (Deuss and Woodhouse, 2001; Deuss et al., 2013). We will expand on the splitting matrix and synthetic seismograms in Section 2.2.

In general, for a given pair of modes with $k$ and $k^{\prime}$, where $k=k^{\prime}$ indicates self-coupling and $k \neq k^{\prime}$ indicates cross-coupling, the complex splitting function coefficients $\sigma_{s t}$ are defined as

$$
\sigma_{s t}^{\left(k k^{\prime}\right)}=c_{s t}^{\left(k k^{\prime}\right)}+\mathrm{i} d_{s t}^{\left(k k^{\prime}\right)}
$$

of which the real part $c_{s t}$ describes the elastic structure, depending on heterogeneous velocity and density structure, and the imaginary part $d_{s t}$ describes the anelastic structure, depending on heterogeneous bulk and shear attenuation. The elastic $c_{s t}$ splitting function coefficients are written as

$$
c_{s t}^{\left(k k^{\prime}\right)}=\int_{0}^{a} \delta m_{s t}(r) \cdot K_{s}^{\left(k k^{\prime}\right)}(r) \mathrm{d} r+\sum_{d} \delta h_{s t}^{d} H_{s}^{d\left(k k^{\prime}\right)}
$$


where $s$ is the angular order and $t$ the azimuthal order of the Earth's structure, and the integral is calculated over $a$, the radius of the Earth. $K_{s}(r)$ and $H_{s}^{d}$ are the kernels derived by Woodhouse (1980); $\delta m_{s t}$ are the coefficients of the Earth's structure (compressional and shear wave velocity $v_{p}, v_{s}$, which can be anisotropic, and density $\rho$ ) and $\delta h_{s t}^{d}$ are the coefficients of discontinuity topography. Both $\delta m_{s t}$ and $\delta h_{s t}^{d}$ can be determined in a linear inversion using the measured splitting function coefficients and the known sensitivity kernels. The anelastic splitting function coefficients $d_{s t}$ are written as

$$
d_{s t}^{\left(k k^{\prime}\right)}=\frac{1}{2} \omega_{0}^{-2} \int_{0}^{a}\left(\kappa_{0} \delta q_{s t}^{\kappa} K_{s}^{q_{\kappa}\left(k k^{\prime}\right)}(r)+\mu_{0} \delta q_{s t}^{\mu} K_{s}^{q_{\mu}\left(k k^{\prime}\right)}(r)\right) r^{2} \mathrm{~d} r
$$

where again $K_{s}^{q_{\kappa}}(r)$ and $K_{s}^{q_{\mu}}(r)$ are the kernels derived by Woodhouse (1980); $\delta q_{s t}^{\kappa}$ are the bulk attenuation structure coefficients and $\delta q_{s t}^{\mu}$ are the shear attenuation structure coefficients; and $\kappa$ is the bulk modulus and $\mu$ is the shear modulus.

Splitting function coefficients (in frequency units) are usually visualised in a map $F(\theta, \phi)$, comparable to phase-velocity maps used in surface wave analysis. These maps show local frequency variations of a specific normal mode, and are calculated using

$$
\begin{aligned}
& F^{E}(\theta, \phi)=\sum_{s=2}^{2 l} \sum_{t=-s}^{s} Y_{s}^{t}(\theta, \phi) c_{s t}^{\left(k k^{\prime}\right)} \\
& F^{A}(\theta, \phi)=\sum_{s=2}^{2 l} \sum_{t=-s}^{s} Y_{s}^{t}(\theta, \phi) d_{s t}^{\left(k k^{\prime}\right)}
\end{aligned}
$$

where $Y_{s}^{t}(\theta, \phi)$ are the fully normalised complex spherical harmonics (Edmonds, 1960) and $(\theta, \phi)$ indicate the surface position. Splitting function measurements also provide constraints on the average $1 \mathrm{D}$ structure seen by a mode. $\operatorname{Re}\left(c_{00}\right)$ constrains the shift in centre frequency $f_{c}$ of a particular mode with respect to the $1 \mathrm{D}$ underlying model. $\operatorname{Re}\left(d_{00}\right)$, also called $\operatorname{Im}\left(c_{00}\right)$, constrains the quality factor $Q$ shift in the same manner

$$
\begin{gathered}
f_{c}=f_{0}+(4 \pi)^{-1 / 2} \operatorname{Re}\left(c_{00}\right) \\
Q=\frac{f_{c}}{2\left(\frac{f_{0}}{2 Q_{0}}+(4 \pi)^{-1 / 2} \operatorname{Re}\left(d_{00}\right)\right)}
\end{gathered}
$$

where $f_{0}$ and $Q_{0}$ are the reference model frequency and quality factor, see Eqs. 12-13 and Eqs. 37-40 in Giardini et al. (1988) for the derivation of these equations, which is equivalent to the diagonal sum rule (Gilbert, 1971).

\subsubsection{Synthetic splitting functions}

We would like to compare our measured splitting functions with predictions computed for existing tomographic models. For this, we will use the shear wave velocity model S20RTS (Ritsema et al., 1999), with a scaling of the form $\delta v_{p} / v_{p}=0.5 \delta v_{s} / v_{s}$ and 
$\delta \rho / \rho=0.3 \delta v_{s} / v_{s}$, together with the crustal structure model CRUST5.1 (Mooney et al., 1998). Here, in order to take into account the influence of the crust on our 1D underlying model, when using first order perturbation theory, we include the effects of topography, water level and crustal thickness. But we do not include the individual layer variations of the crust, because we find they do not show a significant effect in our normal mode observations. However, the crust can have more significant effects when building a tomographic model based on our splitting functions observations, and should be taken into account in more detail (e.g. Karaoğlu and Romanowicz, 2018).

In addition, we use a synthetic 3D $q_{\mu}$ mantle model, by applying the scaling $\delta q_{\mu}=R_{q} \delta v_{s} / v_{s}$ to the $v_{s}$ model S20RTS (Ritsema et al., 1999) to obtain anelastic splitting function predictions, and we also calculate anelastic splitting functions predictions for the model QRSFI12 (Dalton et al., 2008), more details are given in Chapters 5 and 6. Inner core anisotropy is included using the model of Tromp (1993).

\subsection{Synthetic seismograms}

In order to measure the splitting function coefficients of a particular mode or a particular group of modes, we need to calculate their corresponding synthetic seismograms. This is done using summation of modes (e.g. Deuss and Woodhouse, 2001; Akbarashrafi et al., 2017), and outlined in this section. To calculate synthetic seismograms, we first need to solve the momentum equation of the elastic displacement field $\mathbf{s}$, which is a non-homogeneous second-order differential equation

$$
\mathbf{V} \mathbf{s}+\mathbf{W} \frac{\partial}{\partial t} \mathbf{s}+\mathbf{P} \frac{\partial^{2}}{\partial t^{2}} \mathbf{s}=\mathbf{S}
$$

where $\mathbf{V}$ is the potential energy operator related to material properties $\kappa$ and $\mu$ of the Earth and the centrifugal force, $\mathbf{W}$ is the Coriolis force operator, $\mathbf{P}$ is the kinetic force operator related to the Earth's density $\rho$ distribution, and $\mathbf{S}$ is the excitation generated by an earthquake source. This equation is solved by first finding the homogeneous solution, where $\mathbf{S}=\mathbf{0}$, and later using it together with the particular solution to find the general non-homogeneous solution, where the source term $\mathbf{S}$ is non-zero. The final solution will be a time dependent harmonic function of the form

$$
\mathbf{s}(\mathbf{x}, t)=\mathbf{s}(\mathbf{x}) e^{\mathrm{i} \omega t}
$$

where the radial frequency is $\omega$. Although Eq. 2.8 can be solved by approximating $\omega$ around a fiducial frequency $\omega_{0}$, this solution is only valid in small frequency bands. For this reason, in this section we describe a more general solution to the differential equation using variations of parameters. As outlined in Deuss and Woodhouse (2001), three possible solutions exist: (i) exact, (ii) Coriolis approximation and (iii) Coriolis and kinetic energy approximation. Here, we will concentrate on the last, as the differences between the three solutions are negligible, and the Coriolis and kinetic energy approximation allows for both a faster computation time and for every section of the splitting matrix $\mathbf{M}$ to have its own approximated frequency, which we will explain further in Section 2.2.2. The exact solution is given in Section 2.A for completeness. 


\subsubsection{Coriolis and kinetic energy approximation}

The first step is to find the homogeneous solution $\mathbf{K}(t)$ to the homogeneous momentum equation by setting $\mathbf{S}=\mathbf{0}$. Then, we expand $\mathbf{s}$ in terms of basis functions (Woodhouse, 1983) to change the momentum equation Eq. 2.8 into a matrix equation. Applying the Fourier transformation then allows us to rewrite the derivatives as $\partial_{t} \rightarrow \mathrm{i} \omega$ and obtain a non-standard eigenvalue problem which depends on both $\omega$ and $\omega^{2}$

$$
\mathbf{V K}(t)+\mathrm{i} \omega \mathbf{W K}(t)-\omega^{2} \mathbf{P K}(t)=\mathbf{0} .
$$

To reduce the size of the problem, we approximate the frequency dependence in the Coriolis term $\mathbf{W}$ by replacing

$$
\mathrm{i} \omega \mathbf{W} \approx \mathrm{i} \tilde{\omega} \mathbf{W}, \quad \text { with } \quad \tilde{\omega}=\sqrt{\omega_{1} \omega_{2}}
$$

where $\omega_{1}$ and $\omega_{2}$ are the degenerate frequencies of two cross-coupled modes. If there is only one mode of interest (SC case), then $\tilde{\omega}$ is reduced to $\omega_{1}$. In order to avoid having to invert the kinetic energy matrix $\mathbf{P}$, we need to rewrite $\mathbf{P}$. If the Earth had no density perturbations the kinetic energy $\mathbf{P}$ can be modelled as the identity matrix I. On the other hand, if the Earth is non-homogeneous, then the kinetic energy $\mathbf{I}$ will be altered by density perturbations $\mathbf{P}_{1}$, which means $\mathbf{P}$ can be modelled by $\mathbf{I}+\mathbf{P}_{1}$. This transforms the generalised eigenvalue problem to

$$
\begin{aligned}
{[\mathbf{V}+\mathrm{i} \tilde{\omega} \mathbf{W}] \mathbf{K}(t) } & =\omega^{2}\left[\mathbf{I}+\mathbf{P}_{1}\right] \mathbf{K}(t) \\
{\left[\mathbf{V}+\mathrm{i} \tilde{\omega} \mathbf{W}-\omega^{2} \mathbf{P}_{1}\right] \mathbf{K}(t) } & =\omega^{2} \mathbf{K}(t) .
\end{aligned}
$$

Now, we also apply the frequency approximation to the kinetic force

$$
\omega^{2} \mathbf{P}_{1} \approx \tilde{\omega}^{2} \mathbf{P}_{1}, \quad \text { with } \quad \tilde{\omega}^{2}=\omega_{1} \omega_{2}
$$

which reduces our problem to a non-generalised eigenvalue problem of the form

$$
\begin{aligned}
{\left[\mathbf{V}+\mathrm{i} \tilde{\omega} \mathbf{W}-\tilde{\omega}^{2} \mathbf{P}_{1}\right] \mathbf{K}(t) } & =\omega^{2} \mathbf{K}(t) \\
\mathbf{M} \mathbf{K}(t) & =\omega^{2} \mathbf{K}(t)
\end{aligned}
$$

where matrix $\mathbf{M}$ is given by

$$
\mathbf{M}=\mathbf{V}+\mathbf{i} \tilde{\omega} \mathbf{W}-\tilde{\omega}^{2} \mathbf{P}_{1}
$$

This eigenvalue problem has the homogeneous solution $\mathbf{K}(t)=e^{\mathrm{i} \sqrt{\mathbf{M}} t}$, where matrix $\mathbf{M}$ has a dimension equal to $\omega^{2}$. Finally, we diagonalise $\sqrt{\mathbf{M}}$ as $\mathbf{U} \sqrt{\boldsymbol{\Lambda}} \mathbf{U}^{-1}$ and calculate the synthetic seismogram $u=\mathbf{r} \partial_{t}^{2} \mathbf{s}$ using the general solution in Eq. 2.A.42 to get

$$
u(t)=\mathbf{r} \mathbf{U} e^{\mathrm{i} \sqrt{\boldsymbol{\Lambda}} t} \mathbf{U}^{-1} \mathbf{S}
$$

where $\mathbf{r}$ is the receiver vector, which incorporates the instrument response and depends on the instrument orientation. 


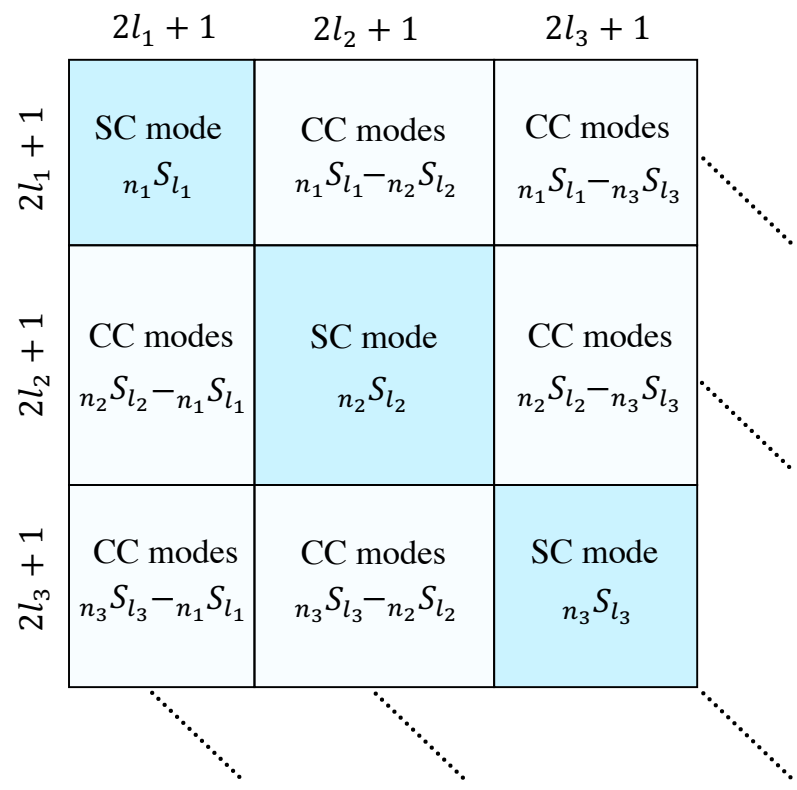

Figure 2.2: Schematic depiction of the splitting matrix for the coupling of modes. On the main diagonal, self-coupling blocks (SC, blue) are of size $\left(2 l_{i}+1\right) \times\left(2 l_{i}+1\right)$, with one existing for each mode ${ }_{n_{i}} S_{l_{i}}$. On the off-diagonal, cross-coupling blocks (CC, grey) are of size $\left(2 l_{i}+1\right) \times\left(2 l_{j}+1\right)$, with two existing for each pair of modes ${ }_{n_{i}} S_{l_{i}-n_{j}} S_{l_{j}}$.

\subsubsection{Splitting matrix}

As seen in the previous section, we write the contribution of a mode to a synthetic seismogram as a harmonic function of time $t$ as

$$
u(t)=\operatorname{Re}\left[\mathbf{r} e^{\mathrm{i} \sqrt{\mathbf{M}} t} \mathbf{S}\right]=\operatorname{Re}\left[\mathbf{r} \mathbf{U} e^{\mathrm{i} \sqrt{\mathbf{\Lambda}} t} \mathbf{U}^{-1} \mathbf{S}\right]
$$

where $\mathbf{r}$ is the receiver vector, dependent on the location and instrument response of the recording seismometer, and $\mathbf{S}$ is the source vector dependent on the moment tensor solution of a particular earthquake. $\mathbf{r}$ and $\mathbf{S}$ are computed with respect to the underlying 1D model used (PREM, Dziewonski and Anderson, 1981).

The splitting matrix $\mathbf{M}$ is a block diagonal complex matrix. The size of $\mathbf{M}$ can be calculated with $\left(2 l_{i}+1\right) \times\left(2 l_{i}+1\right)$, where $l$ is the angular order of a mode and $i$ sums over the number of modes included in $\mathbf{M}$ (Fig. 2.2). The matrix $\mathbf{M}$ contains the self-coupling blocks $\left(l=l^{\prime}\right)$ of each mode present on the diagonal, and the cross-coupling blocks $\left(l \neq l^{\prime}\right)$ between all pairs of modes in the off-diagonal blocks. In the self-coupling (SC) approximation, all off-diagonal blocks are set to zero, resulting in square SC matrices on the main diagonal only, which can be easily diagonalised. On the other hand, group-coupling (GC), and in particular full-coupling (FC), fill up all off-diagonal matrices, resulting in a larger matrix, which is more computationally 
expensive to diagonalise. FC of the 140 normal modes present up-to $3 \mathrm{mHz}$ results in a matrix with a size of $2347 \times 2347$.

The splitting matrix $\mathbf{M}$ contains the degenerate multiplet frequencies $\omega_{0}^{2}$ on the diagonal elements for the self-coupling matrix, in addition to the effects of rotation (including the second-order Coriolis effects, e.g. Dahlen and Sailor, 1979), ellipticity and the splitting coefficients related to heterogeneity for all self-coupling and cross-coupling matrix blocks. For a pair of modes $k k^{\prime}$ we can write $\mathbf{M}$ as

$$
\mathbf{M}_{m m^{\prime}}^{\left(k k^{\prime}\right)}=\omega_{0}^{2} \delta_{m m^{\prime}}^{\left(k k^{\prime}\right)}+\omega_{0} \mathbf{W}_{m m^{\prime}}^{\left(k k^{\prime}\right)}+\mathbf{H}_{m m^{\prime}}^{\left(k k^{\prime}\right)}
$$

where $\delta_{k k^{\prime}}=0$ if $k \neq k^{\prime}$ and $\delta_{k k^{\prime}}=1$ if $k=k^{\prime}$ and $\omega_{0}=\left(\omega_{0}^{k}+\omega_{0}^{k^{\prime}}\right) / 2$. The matrix $\mathbf{W}$ contains the Coriolis force effect, and the splitting matrix $\mathbf{H}$ contains the potential energy $\mathbf{V}$ and the density perturbations $\mathbf{P}_{1}$, as described in Section 2.2.1. In this way, all contribution from 3D heterogeneity in velocity, density, attenuation and anisotropy are now all contained in the matrix $\mathbf{H}$.

$\mathbf{H}$ can be further separated in the elastic heterogeneity matrix $\mathbf{H}^{E}$ and the anelastic heterogeneity matrix $\mathbf{H}^{A}$

$$
\mathbf{H}_{m m^{\prime}}^{\left(k k^{\prime}\right)}=\mathbf{H}_{m m^{\prime}}^{E\left(k k^{\prime}\right)}+\mathrm{i} \mathbf{H}_{m m^{\prime}}^{A\left(k k^{\prime}\right)}
$$

Following Li et al. (1991) and Mäkinen and Deuss (2013), matrices $\mathbf{H}^{E}$ and $\mathbf{H}^{A}$ can be expanded as

$$
\mathbf{H}_{m m^{\prime}}^{\left(k k^{\prime}\right)}=\sum_{s=0}^{2 l} \sum_{t=-s}^{s} \gamma_{l l^{\prime} s}^{m m^{\prime} t} c_{s t}^{\left(k k^{\prime}\right)}+\mathrm{i} \sum_{s=0}^{2 l} \sum_{t=-s}^{s} \gamma_{l l^{\prime} s}^{m m^{\prime} t} d_{s t}^{\left(k k^{\prime}\right)}
$$

where $c_{s t}$ and $d_{s t}$ are the elastic and anelastic splitting coefficients receptively, and $s$ is the angular order and $t$ the azimuthal order of the Earth's structure. The $\gamma_{l l^{\prime} s}^{m m^{\prime} t}$ coefficients are written as

$$
\gamma_{l l^{\prime} s}^{m m^{\prime} t}=\int_{0}^{2 \pi} \int_{0}^{\pi} Y_{l}^{m^{*}}(\theta, \phi) Y_{s}^{t}(\theta, \phi) Y_{l}^{m^{\prime}}(\theta, \phi) \sin \theta \mathrm{d} \theta \mathrm{d} \phi
$$

where $(\theta, \phi)$ indicates the surface position and ${ }^{*}$ is the complex conjugate notation. $Y_{l}^{m}$ are the fully normalised complex spherical harmonics (Edmonds, 1960) defined as

$$
Y_{m}^{l}(\theta, \phi)=(-1)^{m}\left[\frac{(2 l+1)}{4 \pi} \frac{(l-m) !}{(l+m) !}\right]^{1 / 2} P_{l}^{m}(\cos \theta) e^{\mathrm{i} m \phi}
$$

with $l=0,1 \ldots$ and $m=-l,-l+1, \ldots, l . P_{l}^{m}$ are the associated Legendre functions

$$
P_{l}^{m}(x)=\frac{\left(1-x^{2}\right)^{m / 2}}{2^{l} l !} \frac{\mathrm{d}^{l+m}}{\mathrm{~d} x^{l+m}}\left(x^{2}-1\right)^{l}
$$

We include Earth's ellipticity as an additional degree $s=2, t=0$ term. Eqs. 2.18-2.21 are reduced to self-coupling equations in the cases where $k=k^{\prime}$, and therefore $l=l^{\prime}$. 


\subsubsection{Linearising the problem: calculating derivatives}

The synthetic seismograms $u$ have a non-linear dependence on the splitting function coefficients $c_{s t}$ and $d_{s t}$. In order to set up a linearised and iterative inverse problem, we first need to calculate the derivatives of the synthetic seismogram (Eq. 2.16) with respect to $c_{s t}$ and $d_{s t}$. Within the synthetic seismogram calculation, the eigenvectors $\mathbf{U}, \mathbf{U}^{-1}$ and the eigenvalues $\sqrt{\boldsymbol{\Lambda}}$ of the splitting matrix $\mathbf{M}$ all depend on the splitting function coefficients. Thus, the full partial derivatives are given by

$$
\begin{aligned}
& \frac{\partial u(t)}{\partial c_{s t}}=\operatorname{Re}\left\{e^{\mathbf{i} \omega t}\left[\left(\mathbf{r} \frac{\partial \mathbf{U}}{\partial c_{s t}}\right)\left(\mathbf{U}^{-1} \mathbf{s}\right)+(\mathbf{r} \mathbf{U})\left(\frac{\partial \mathbf{U}^{-1}}{\partial c_{s t}} \mathbf{s}\right)+\mathrm{i} t(\mathbf{r} \mathbf{U}) \frac{\partial \omega}{\partial c_{s t}}\left(\mathbf{U}^{-1} \mathbf{s}\right)\right]\right\} \\
& \frac{\partial u(t)}{\partial d_{s t}}=\operatorname{Re}\left\{e^{\mathbf{i} \omega t}\left[\left(\mathbf{r} \frac{\partial \mathbf{U}}{\partial d_{s t}}\right)\left(\mathbf{U}^{-1} \mathbf{s}\right)+(\mathbf{r} \mathbf{U})\left(\frac{\partial \mathbf{U}^{-1}}{\partial d_{s t}} \mathbf{s}\right)+\mathrm{i} t(\mathbf{r} \mathbf{U}) \frac{\partial \omega}{\partial d_{s t}}\left(\mathbf{U}^{-1} \mathbf{s}\right)\right]\right\}
\end{aligned}
$$

where $\omega=\sqrt{\boldsymbol{\Lambda}}$ is a diagonal matrix containing all the eigenvalues of of $\mathbf{M}$. The splitting coefficients perturbations $\partial c_{s t}$ and $\partial d_{s t}$ cause perturbations $\delta \mathbf{M}$ to the splitting matrix. We use Rayleigh's principle to calculate the changes in $\delta \boldsymbol{\Lambda}$ and $\delta \mathbf{U}$ in terms of the unperturbed eigenfunctions $\left({ }_{n} U_{l}\right.$ and $\left.{ }_{n} V_{l},{ }_{n} W_{l}\right)$, without the need to solve the perturbed eigenfunctions in parallel (Dahlen and Tromp, 1998). These results in the following equations for the changes in eigenfrequencies and eigenvectors due to a change in the splitting functions coefficients,

$$
\begin{array}{rlrl}
\delta \omega_{i}^{2} & =\mathbf{u}_{i}^{-1} \delta \mathbf{M}\left(\delta c_{s t}\right) \mathbf{u}_{i} ; & \delta \omega_{i}^{2} & =\mathbf{u}_{i}^{-1} \delta \mathbf{M}\left(\delta d_{s t}\right) \mathbf{u}_{i} \\
\delta \mathbf{u}_{i} & =\sum_{j \neq i} \frac{\mathbf{u}_{j}^{-1} \delta \mathbf{M}\left(\delta c_{s t}\right) \mathbf{u}_{i}}{\omega_{i}^{2}-\omega_{j}^{2}} \mathbf{u}_{j} ; & \delta \mathbf{u}_{i} & =\sum_{j \neq i} \frac{\mathbf{u}_{j}^{-1} \delta \mathbf{M}\left(\delta d_{s t}\right) \mathbf{u}_{i}}{\omega_{i}^{2}-\omega_{j}^{2}} \mathbf{u}_{j} \\
\delta \mathbf{u}_{i}^{-1} & =\sum_{j \neq i} \frac{\mathbf{u}_{i}^{-1} \delta \mathbf{M}\left(\delta c_{s t}\right) \mathbf{u}_{j}}{\omega_{i}^{2}-\omega_{j}^{2}} \mathbf{u}_{j}^{-1} ; & \delta \mathbf{u}_{i}^{-1}=\sum_{j \neq i} \frac{\mathbf{u}_{i}^{-1} \delta \mathbf{M}\left(\delta d_{s t}\right) \mathbf{u}_{j}}{\omega_{i}^{2}-\omega_{j}^{2}} \mathbf{u}_{j}^{-1}
\end{array}
$$

where index $i$ is related to the number of eigenfrequencies and eigenvectors of a multiplet, and $\omega_{i}$ is a diagonal element of $\omega=\sqrt{\Lambda}$, and $\mathbf{u}_{i}$ represents a column vector of $\mathbf{U}$, and $\mathbf{u}_{i}^{-1}$ is a row vector of $\mathbf{U}$. We use these changes in $\delta \boldsymbol{\Lambda}, \delta \mathbf{U}$ and $\delta \mathbf{U}^{-1}$ to replace the terms $\frac{\partial \mathbf{U}}{\partial c_{s t}}, \frac{\partial \mathbf{U}^{-1}}{\partial c_{s t}}, \frac{\partial \omega}{\partial c_{s t}}$ in Eq. 2.24 and $\frac{\partial \mathbf{U}}{\partial d_{s t}}, \frac{\partial \mathbf{U}^{-1}}{\partial d_{s t}}, \frac{\partial \omega}{\partial d_{s t}}$ in Eq. 2.25. 


\subsection{Inverse problem}

In this thesis, our aim is to retrieve the splitting function coefficients $c_{s t}$ and $d_{s t}$ from observed normal mode spectra. The inversion scheme used to measure the splitting function coefficients is the damped iterated least-squares method of Tarantola and Valette, 1982, which in combination with the forward problem setup described in Section 2.2 is referred in the literature as iterative spectral fitting. This is done in the same manner as Deuss et al. (2013), by applying the recursion

$$
\mathbf{m}^{i+1}=\mathbf{m}^{i}+\left(\mathbf{A}_{i}^{T} \mathbf{C}_{d}^{-1} \mathbf{A}_{i}+\mathbf{C}_{m}^{-1}\right)^{-1}\left(\mathbf{A}_{i}^{T} \mathbf{C}_{d}^{-1}\left(\mathbf{d}^{o b s}-\mathbf{u}^{i}\right)-\mathbf{C}_{m}^{-1}\left(\mathbf{m}^{i}-\mathbf{m}^{0}\right)\right)
$$

where the matrix $\mathbf{A}$, contains the partial derivatives of the synthetic seismograms (Eqs. 2.24-2.25), and can be written as

$$
\mathbf{A}_{i}=\left[\frac{\partial \mathbf{u}}{\partial \mathbf{m}}\right]_{\mathbf{m}=\mathbf{m}^{i}}
$$

The model vector $\mathbf{m}^{i}=\left(\begin{array}{ll}\mathbf{c}^{i} & \mathbf{d}^{i}\end{array}\right)^{T}$ contains the elastic splitting function model vector $\mathbf{c}^{i}$ and anelastic splitting function model vector $\mathbf{d}^{i}$ for a particular mode or group of modes, $\mathbf{m}^{0}=\left(\begin{array}{ll}\mathbf{c}^{0} & \mathbf{d}^{0}\end{array}\right)^{T}$ is the starting model, $\mathbf{d}^{\text {obs }}$ is the observed spectral data vector, $\mathbf{u}^{i}$ is the synthetic seismogram calculated with Eq. 2.16 using the model vector $\mathbf{m}^{i}=\left(\begin{array}{ll}\mathbf{c}^{i} & \mathbf{d}^{i}\end{array}\right)^{T} . \mathbf{C}_{d}$ is the a priori data covariance matrix, and $\mathbf{C}_{m}$ is the splitting function model covariance matrix.

We assume that both covariance matrices are diagonal with a constant variances $\sigma_{d}^{2}$ and $\sigma_{m}^{2}$, and that they are the same for all spectral data and model parameters in a given splitting function inversion. Given this, we can rewrite the recursive damped least-squares equation in terms of the ratio $\sigma_{d}^{2} / \sigma_{m}^{2}$

$$
\mathbf{m}^{i+1}=\mathbf{m}^{i}+\left(\mathbf{A}_{i}^{T} \mathbf{A}_{i}+\frac{\sigma_{d}^{2}}{\sigma_{m}^{2}} \mathbf{I}\right)^{-1}\left(\mathbf{A}_{i}^{T}\left(\mathbf{d}^{o b s}-\mathbf{u}^{i}\right)-\frac{\sigma_{d}^{2}}{\sigma_{m}^{2}} \mathbf{I}\left(\mathbf{m}^{i}-\mathbf{m}^{0}\right)\right)
$$

Now we call the ratio $\sigma_{d}^{2} / \sigma_{m}^{2}=\eta$ the norm damping; it can also be referred as a trade-off or regularisation parameter. Because $\eta$ provides the ratio $\sigma_{d}^{2} / \sigma_{m}^{2}$, it helps us determine the trade-off between minimising the model (i.e. splitting function) coefficients and their misfit to the data. The smaller $\eta$ is, the less damping is applied. A range of damping parameters $\eta$ across different orders of magnitudes are used in order to determine the best value of $\eta$ for each splitting function inversion. This optimal damping is determined by seeing how the misfit of the model to the real data, the independent number of parameters and the squared model size vary as a function of damping and iteration. Just like in Deuss et al. (2013), who did not find an improvement in their results by varying damping according the angular order

Matrix $\mathbf{A}_{i}^{T} \mathbf{A}_{i}$ in Eq. 2.31 is commonly referred to as the 'Hessian' in the literature, although it is only an approximation to the true complete Hessian matrix. 
of the splitting function coefficients (i.e. derivative damping), we apply the same damping to all model parameters.

The posteriori resolution matrix $\mathbf{R}$ is defined in the neighbourhood of the final model $\mathbf{c}_{\infty}$ and $\mathbf{d}_{\infty}$. This means our starting model need to be in the vicinity of the final model to give meaning to $\mathbf{R}$ and have a converging final model. The resolution matrix $\mathbf{R}$ is defined as

$$
\mathbf{R}=\left(\mathbf{A}_{\infty}^{T} \mathbf{C}_{d}^{-1} \mathbf{A}_{\infty}+\mathbf{C}_{m}^{-1}\right)^{-1} \mathbf{A}_{\infty}^{T} \mathbf{C}_{d}^{-1} \mathbf{A}_{\infty}
$$

We use the trace of $\mathbf{R}$ to provide us with the number of effective eigenvalues or independent number of parameters in the new model. Finally, we also need to define the misfit $\chi$ between the data $\mathbf{d}_{i}^{\text {obs }}$ and the synthetics $\mathbf{u}_{i}$ in order to judge how well our splitting function measurements fit the original spectral data. This misfit is given as

$$
\chi=\frac{1}{N} \sum^{N} \frac{\sum_{i=1}^{n}\left|\mathbf{d}_{i}^{o b s}-\mathbf{u}_{i}\right|^{2}}{\sum_{i=1}^{n}\left|\mathbf{d}_{i}^{\text {obs }}\right|^{2}}
$$

where is $N$ is the total number of spectral segments ${ }^{1}$ used for a specific mode in the inversion, and $n$ are the number of data points contained within each spectral segment. The misfit is calculated using both the phase and amplitude of the spectral data.

\subsubsection{Uncertainties}

Unlike Pachhai et al. (2020), who uses the auto-regressive matrix method together with a Bayesian inversion and Metropolis Hasting Sampling in order to obtain the probabilistic distributions of their measurements, we calculate the uncertainties of our measurements by doing event cross-validation. We do this by removing whole events at the time (e.g. 1994 Bolivia or 2011 Tohoku events), including all stations, and measuring what the splitting function would be without this event. In this way, we estimate earthquake bias (e.g. Deuss et al., 2013) and obtain uncertainties for our measured splitting function coefficients. Because we realise that our calculated uncertainties underestimate the real uncertainties (Akbarashrafi et al., 2017), we report the differences between our observations and the upper and lower bound coefficients obtained through the cross-validation, which results asymmetrical uncertainties in some cases.

\subsubsection{F-tests}

In order to determine if a misfit reduction is statistically significant between inversions using different parameterisations, we use F-test statistics, which allow us to directly compare two observations (e.g. Mäkinen and Deuss, 2013; Koelemeijer et al., 2013). To evaluate the F-test, we select a certain confidence level $\alpha$, and we use the null hypothesis that there is no significant misfit reduction when increasing the number

\footnotetext{
${ }^{1}$ We defined a spectral segment as the combination of the particular frequency window and time window used to measure a particular mode.
} 
of parameters in a model. To test if the null hypothesis holds, we compare the F-test critical value to the F-distribution (e.g. Abramowitz and Stegun, 1965). If the critical F-value exceeds the F-distribution value, we can reject the null hypothesis with a $(1-\alpha) \%$ confidence level. The critical $\mathrm{F}$-test value is calculated using

$$
F=\frac{\left(\frac{m_{1}-m_{2}}{p_{2}-p_{1}}\right)}{\left(\frac{m_{2}}{n-p_{2}}\right)}
$$

where $p_{1}$ is the number of parameters in the simpler model (e.g. SC, elastic coefficients), $p_{2}$ is the number of parameters in the more complex model (e.g. GC, elastic and anelastic coefficients), meaning $p_{2}$ is always larger than $p_{1}$, while $m_{1}$ and $m_{2}$ are the misfits associated to each model, and $n$ is the number of spectra used in the inversion. We will use the F-test to evaluate the significance of our misfit reductions in Chapter 4, where we compare SC and GC measurements, and in Chapter 6, where we compare purely elastic observations to anelastic observations.

\subsection{Data}

Normal mode observations require several day-long seismic data of large earthquakes $\left(M_{w}>7.4\right)$ with a high signal-to-noise ratio (ideally above 2$)$, to make their discrete frequencies visible as distinct peaks in the frequency spectrum (Fig. 2.3). Here, we will use 104 of the largest events available from the last 42 years (given in Table 2.1 and shown on a map in Fig. 2.4) to invert for normal mode splitting function coefficients. We are including 14 new events compared to the events used in Deuss et al. (2013) and Koelemeijer et al. (2013), but excluded the 2004 great Sumatra earthquake due to its complicated moment tensor solution and long source duration which becomes especially problematic when investigating attenuation. Earthquakes preceded or succeed by another event within a week, and with a moment magnitude difference of up-to 1.5 are excluded. There might also be an influence of the chosen networks and stations on our measurements (Majstorović et al., 2019). We did not see any evidence for network problems, but for completeness all stations and networks used in our study are given in Table 2.2.

The normal mode spectra are obtained by Fourier transforming the time-window data to the frequency domain, so the details of the time window are important. To select the data time windows for the Fourier transformation, we use the $Q$-cycle of each mode as a reference (Dahlen, 1982). The $Q$-cycle is defined as the quality factor $Q$ of a mode divided by its frequency in $\mathrm{Hz}$, and is the time it takes a wave to decay to $e^{-\pi}$ of its original amplitude. It has been suggested that $1.1 \times Q$-cycle would be the optimal time-window to perform normal mode observations (Dahlen, 1982). In addition, the first few hours after the event time were excluded for all modes, in order to: (i) remove body waves, which arrive in the first hour or so of the event and are like delta pulses that lead to noise in the frequency domain, interfering with the spectral data; and, in the case of inner core sensitive modes, (ii) to isolate the mode of interest in our frequency window and remove the effect of strongly attenuating modes with similar frequencies, but much smaller $Q$-cycles. For most 
$M_{w} 8.2$ Bolivia Earthquake, 1994

Station HRV, 5-50 hrs

${ }_{2} T_{2}$

${ }_{0} S_{14}$

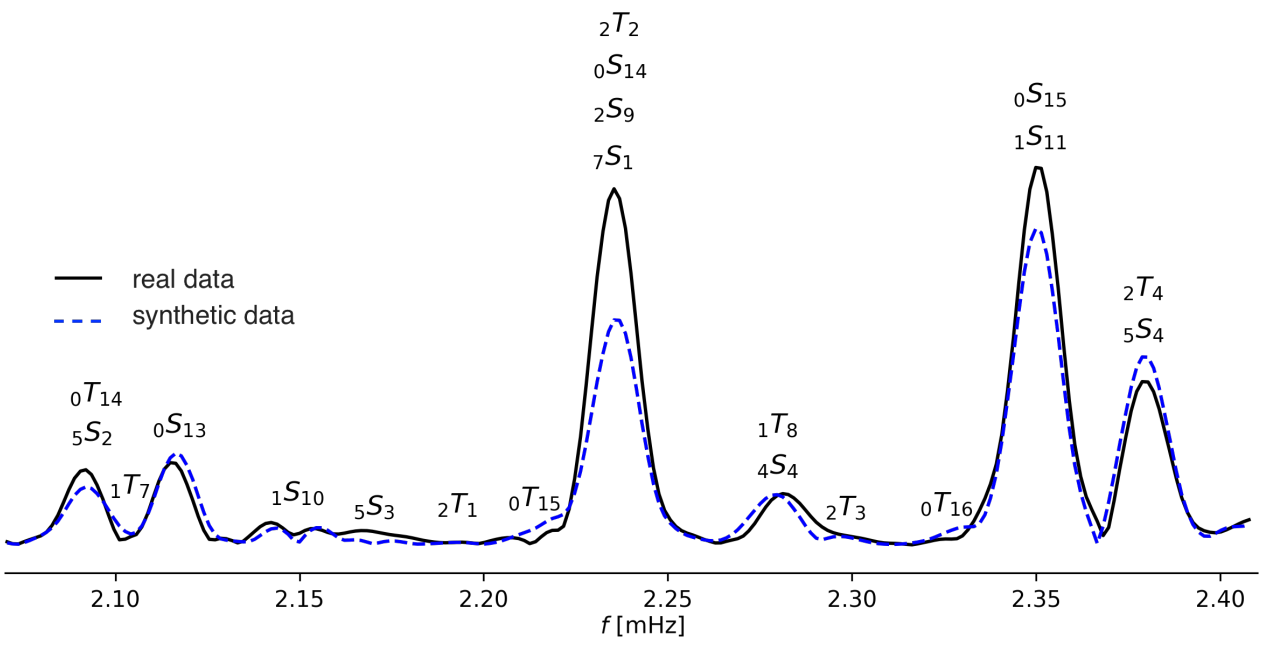

Figure 2.3: Vertical component data of the $M_{w} 8.3$ Bolivia earthquake of 1994 for station HRV with a time window of 5-50 h. Observed spectral amplitude data (black) is compared to the 1D fully coupled synthetics predictions (blue) for rotation and ellipticity of PREM (Dziewonski and Anderson, 1981). Modes present in the frequency band are labelled.

modes, a maximum of 3 to 10 hours was excluded. However, for the longest period and most slowly attenuating radial modes in close proximity with inner core modes, several tens of hours had to be excluded. Additionally, we do not use earthquakes that have similar magnitude earthquakes appearing within time-window of interest. In the particular case of radial modes, we only use the largest and deepest events in Fig. 2.4 and Table 2.1. See Section 4.3 for an extensive explanation.

Glitches were removed manually from the time domain seismograms. The tidal signal has been removed, by fitting sine functions to tidal-wave frequencies and subtracting the corresponding long-period signal from the seismograms, and the instrument response was deconvolved. The selected time-series was then tapered, padded with zeros to the next power of 2 and Fourier transformed to obtain spectral data in the frequency domain. All generated synthetic data is processed in the same way as the real data, following the same steps outlined by Deuss et al. (2013). Vertical component data is used for all modes measured in this thesis, while horizontal component data is only used for a few mantle sensitive modes in Chapters 5 and 6 . 


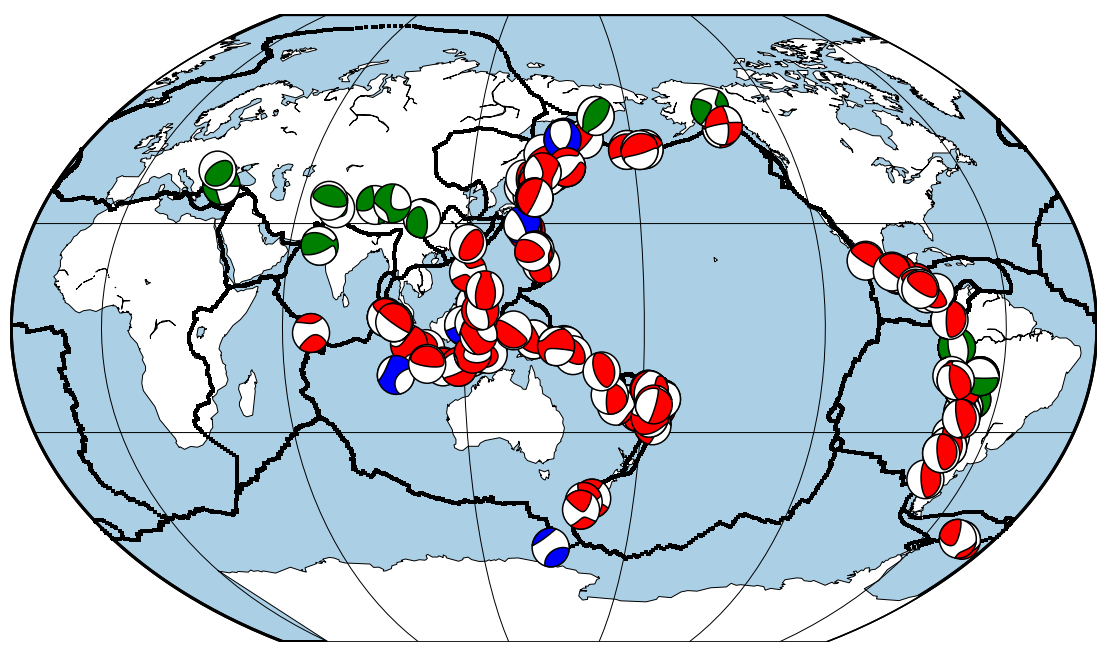

Figure 2.4: Earthquake catalogue. Moment tensor solutions (beachballs) for earthquake in continents (green), oceanic ridges or trenches (red) and ocean floors (blue).

Table 2.1: Earthquakes catalogue. The date format is day/month/year, depth is in $\mathrm{km}$, $M_{w}$ is the moment magnitude as listed in the CMT catalogue. $N$ is the number of stations available per event for the vertical $Z$, transverse $T$ and radial $R$ components. Earthquakes used here to measure inner core sensitive modes are marked with *

\begin{tabular}{clrrrrrl}
\hline Date & Location & Depth & $M_{w}$ & $N_{Z}$ & $N_{T}$ & $N_{R}$ & \\
\hline $19 / 08 / 2018$ & Fiji Islands & 555.0 & 8.2 & 31 & 16 & 10 & \\
$08 / 09 / 2017$ & Chiapas, Mexico & 50.2 & 8.2 & 63 & 4 & 6 & \\
$25 / 12 / 2016$ & Southern Chile & 32.8 & 7.6 & 30 & 5 & 5 & \\
$29 / 07 / 2016$ & Mariana Islands & 208.9 & 7.7 & 62 & 11 & 18 & \\
$26 / 10 / 2015$ & Afghanistan & 209.4 & 7.5 & 33 & 2 & 3 & \\
$16 / 09 / 2015$ & Central Chile & 17.4 & 8.3 & 112 & 9 & 24 & \\
$30 / 05 / 2015$ & Bonin Islands Japan & 680.7 & 7.9 & 76 & 47 & 86 & $*$ \\
$05 / 05 / 2015$ & New Britain, P.N.G. & 38.3 & 7.5 & 15 & 5 & 9 & \\
$17 / 11 / 2013$ & Scotia Sea & 23.8 & 7.8 & 102 & 11 & 11 & \\
$24 / 05 / 2013$ & Sea of Okhotsk & 611.0 & 8.3 & 105 & 91 & 100 & $*$ \\
$05 / 09 / 2012$ & Costa Rica & 29.7 & 7.6 & 93 & 9 & 14 & \\
$31 / 08 / 2012$ & Philippine Island & 45.2 & 7.6 & 99 & 5 & 13 & \\
$27 / 08 / 2012$ & Central America & 12.0 & 7.3 & 58 & 8 & 16 & \\
$14 / 08 / 2012$ & Sea of Okhotsk & 598.2 & 7.7 & 74 & 14 & 13 & \\
$11 / 03 / 2011$ & Tohoku, Japan & 20.0 & 9.1 & 139 & 19 & 144 & $*$ \\
$27 / 02 / 2010$ & Central Chile & 23.2 & 8.8 & 129 & 84 & 24 & $*$ \\
$05 / 07 / 2008$ & Sea of Okhotsk & 610.8 & 7.7 & 64 & 83 & 78 & $*$ \\
$12 / 05 / 2008$ & Sichuan, China & 12.8 & 7.9 & 72 & 89 & 94 & $*$ \\
$09 / 12 / 2007$ & South of Fiji Islands & 149.9 & 7.8 & 48 & 71 & 70 & $*$ \\
$14 / 11 / 2007$ & Northern Chile & 37.6 & 7.7 & 50 & 58 & 60 & \\
\hline
\end{tabular}

Continued on next page 
Table 2.1 - Continued from previous page

\begin{tabular}{|c|c|c|c|c|c|c|c|}
\hline Date & Location & Depth & $M_{w}$ & $N_{Z}$ & $N_{T}$ & $N_{R}$ & \\
\hline $28 / 09 / 2007$ & Volcano Islands & 275.8 & 7.5 & 54 & 47 & 47 & \\
\hline $15 / 08 / 2007$ & Coast of Peru & 33.8 & 8.0 & 53 & 74 & 70 & $*$ \\
\hline $08 / 08 / 2007$ & Java, Indonesia & 304.8 & 7.5 & 49 & 59 & 46 & * \\
\hline $01 / 04 / 2007$ & Solomon Islands & 14.1 & 8.1 & 52 & 77 & 89 & * \\
\hline $13 / 01 / 2007$ & Kuril Islands & 12.0 & 8.1 & 58 & 82 & 87 & * \\
\hline $15 / 11 / 2006$ & Kuril Islands & 13.5 & 8.3 & 69 & 95 & 111 & * \\
\hline $17 / 07 / 2006$ & South of Java & 20.0 & 7.7 & 60 & 55 & 61 & * \\
\hline $03 / 05 / 2006$ & Tonga Islands & 67.8 & 8.0 & 43 & 70 & 74 & * \\
\hline $20 / 04 / 2006$ & Eastern Siberia & 12.0 & 7.6 & 66 & 63 & 68 & * \\
\hline $27 / 01 / 2006$ & Banda Sea & 397.4 & 7.6 & 74 & 63 & 69 & * \\
\hline $08 / 10 / 2005$ & Pakistan & 12.0 & 7.6 & 80 & 68 & 73 & * \\
\hline $26 / 09 / 2005$ & Northern Peru & 108.1 & 7.5 & 63 & 61 & 59 & * \\
\hline 09/09/2005 & New Ireland & 83.6 & 7.6 & 59 & 59 & 62 & * \\
\hline $28 / 03 / 2005$ & Northern Sumatra & 25.8 & 8.6 & 91 & 110 & 163 & * \\
\hline $23 / 12 / 2004$ & Macquarie Islands & 27.5 & 8.1 & 87 & 0 & 33 & * \\
\hline $17 / 11 / 2003$ & Rat Islands & 21.7 & 7.7 & 60 & 62 & 66 & * \\
\hline $25 / 09 / 2003$ & Hokkaido, Japan & 28.2 & 8.3 & 90 & 20 & 20 & * \\
\hline $04 / 08 / 2003$ & Scotia Sea & 15.0 & 7.6 & 61 & 72 & 68 & * \\
\hline $15 / 07 / 2003$ & Carlsberg Ridge & 15.0 & 7.5 & 64 & 61 & 70 & \\
\hline $03 / 11 / 2002$ & Central Alaska & 15.0 & 7.8 & 73 & 72 & 81 & * \\
\hline $08 / 09 / 2002$ & Papua New Guinea & 19.5 & 7.6 & 50 & 51 & 54 & \\
\hline $19 / 08 / 2002$ & South of Fiji & 699.3 & 7.7 & 58 & 69 & 70 & * \\
\hline $14 / 11 / 2001$ & Qinghai China & 15.0 & 7.8 & 65 & 67 & 67 & * \\
\hline $07 / 07 / 2001$ & Coast of Peru & 25.0 & 7.6 & 44 & 53 & 51 & * \\
\hline $23 / 06 / 2001$ & Coast of Peru & 29.6 & 8.4 & 88 & 79 & 81 & * \\
\hline $26 / 01 / 2001$ & India & 19.8 & 7.6 & 66 & 50 & 62 & * \\
\hline $13 / 01 / 2001$ & El Salvador & 56.0 & 7.7 & 61 & 60 & 66 & \\
\hline $18 / 06 / 2000$ & South Indian Ocean & 15.0 & 7.9 & 88 & 79 & 87 & * \\
\hline $04 / 06 / 2000$ & Southern Sumatra & 43.9 & 7.8 & 79 & 67 & 72 & * \\
\hline $28 / 03 / 2000$ & Volcano Islands & 99.7 & 7.6 & 63 & 55 & 62 & * \\
\hline 20/09/1999 & Taiwan & 21.2 & 7.6 & 67 & 36 & 33 & * \\
\hline $17 / 08 / 1999$ & Turkey & 17.0 & 7.6 & 63 & 56 & 51 & \\
\hline $29 / 11 / 1998$ & Ceram Sea & 16.4 & 7.7 & 53 & 51 & 41 & \\
\hline $25 / 03 / 1998$ & Balleny Islands & 28.8 & 8.1 & 66 & 77 & 71 & * \\
\hline $04 / 01 / 1998$ & Loyalty Islands & 114.3 & 7.4 & 35 & 25 & 39 & * \\
\hline $05 / 12 / 1997$ & Kamchatka & 33.6 & 7.8 & 41 & 51 & 64 & * \\
\hline $08 / 11 / 1997$ & Tibet & 16.4 & 7.5 & 61 & 36 & 44 & \\
\hline $14 / 10 / 1997$ & South of Fiji Islands & 165.9 & 7.7 & 43 & 28 & 32 & * \\
\hline $12 / 11 / 1996$ & Coast of Peru & 37.4 & 7.7 & 59 & 40 & 43 & \\
\hline $17 / 06 / 1996$ & Flores Sea & 584.2 & 7.8 & 53 & 70 & 90 & * \\
\hline $10 / 06 / 1996$ & Andreanof Islands & 29.0 & 7.9 & 42 & 17 & 15 & * \\
\hline $17 / 02 / 1996$ & West Irian & 15.0 & 8.2 & 70 & 31 & 46 & * \\
\hline $01 / 01 / 1996$ & Minahassa & 15.0 & 7.9 & 30 & 37 & 33 & * \\
\hline $03 / 12 / 1995$ & Kuril Islands & 25.9 & 7.9 & 47 & 35 & 41 & * \\
\hline $09 / 10 / 1995$ & Jalisco, Mexico & 15.0 & 8.0 & 46 & 33 & 49 & * \\
\hline $30 / 07 / 1995$ & Northern Chile & 28.7 & 8.0 & 50 & 49 & 56 & * \\
\hline
\end{tabular}

Continued on next page 
Table 2.1 - Continued from previous page

\begin{tabular}{|c|c|c|c|c|c|c|c|}
\hline Date & Location & Depth & $M_{w}$ & $N_{Z}$ & $N_{T}$ & $N_{R}$ & \\
\hline $28 / 12 / 1994$ & Coast of Honshu & 27.7 & 7.7 & 50 & 30 & 33 & * \\
\hline 04/10/1994 & Kuril Islands & 68.2 & 8.3 & 46 & 52 & 57 & * \\
\hline 09/06/1994 & Northern Bolivia & 647.1 & 8.2 & 56 & 46 & 57 & * \\
\hline 02/06/1994 & South of Java & 15.0 & 7.8 & 33 & 17 & 24 & \\
\hline 09/03/1994 & Fiji Islands & 567.8 & 7.6 & 54 & 27 & 31 & \\
\hline $12 / 07 / 1993$ & Hokkaido, Japan & 16.5 & 7.7 & 46 & 30 & 31 & * \\
\hline $15 / 01 / 1993$ & Hokkaido, Japan & 100.0 & 7.6 & 27 & 15 & 20 & * \\
\hline $12 / 12 / 1992$ & Flores Island & 20.4 & 7.7 & 36 & 23 & 25 & * \\
\hline $11 / 10 / 1992$ & Vanuatu & 141.1 & 7.4 & 32 & 11 & 16 & * \\
\hline 02/09/1992 & Nicaragua & 15.0 & 7.6 & 30 & 18 & 22 & \\
\hline $22 / 12 / 1991$ & Kuril Islands & 31.2 & 7.6 & 17 & 6 & 10 & * \\
\hline $22 / 04 / 1991$ & Costa Rica & 15.0 & 7.6 & 24 & 8 & 6 & \\
\hline $30 / 12 / 1990$ & New Britain, P.N.G. & 204.8 & 7.4 & 14 & 3 & 5 & * \\
\hline $16 / 07 / 1990$ & Luzon, Philippines & 15.0 & 7.7 & 21 & 6 & 7 & \\
\hline $18 / 04 / 1990$ & Minahassa & 33.2 & 7.6 & 24 & 2 & 7 & * \\
\hline $03 / 03 / 1990$ & South of Fiji & 25.3 & 7.6 & 23 & 1 & 3 & \\
\hline 06/03/1988 & Gulf of Alaska & 15.0 & 7.7 & 19 & 3 & 5 & \\
\hline $30 / 11 / 1987$ & Gulf of Alaska & 15.0 & 7.8 & 19 & 0 & 2 & \\
\hline $20 / 10 / 1986$ & Kermadec Islands & 50.4 & 7.7 & 11 & 0 & 6 & * \\
\hline $07 / 05 / 1986$ & Andreanof Islands & 31.3 & 7.9 & 11 & 2 & 3 & \\
\hline $03 / 03 / 1985$ & Central Chile & 40.7 & 7.9 & 11 & 0 & 2 & \\
\hline $20 / 11 / 1984$ & Philippines & 180.7 & 7.5 & 12 & 0 & 0 & * \\
\hline $06 / 03 / 1984$ & Honshu Japan & 446.0 & 7.4 & 7 & 0 & 1 & * \\
\hline $24 / 11 / 1983$ & Banda Sea & 157.1 & 7.4 & 10 & 1 & 1 & * \\
\hline $04 / 10 / 1983$ & North Chile & 38.7 & 7.6 & 9 & 0 & 0 & \\
\hline $26 / 05 / 1983$ & Honshu, Japan & 12.6 & 7.7 & 12 & 0 & 0 & \\
\hline 18/03/1983 & New Ireland & 69.9 & 7.7 & 15 & 0 & 0 & * \\
\hline $22 / 06 / 1982$ & Banda Sea & 473.4 & 7.4 & 9 & 0 & 0 & * \\
\hline $25 / 05 / 1981$ & South Island, N.Z. & 33.3 & 7.6 & 12 & 0 & 0 & \\
\hline $17 / 07 / 1980$ & Sta. Cruz Islands & 34.0 & 7.7 & 9 & 0 & 0 & \\
\hline $12 / 12 / 1979$ & Coast of Ecuador & 19.7 & 8.1 & 9 & 0 & 0 & * \\
\hline $06 / 12 / 1978$ & Kuril Islands & 181.0 & 7.8 & 11 & 0 & 0 & \\
\hline $29 / 11 / 1978$ & Oaxaca, Mexico & 16.1 & 7.7 & 8 & 0 & 0 & \\
\hline $12 / 06 / 1978$ & Honshu, Japan & 37.7 & 7.6 & 3 & 0 & 0 & * \\
\hline $19 / 08 / 1977$ & Sumbawa Island & 23.3 & 8.3 & 7 & 0 & 0 & * \\
\hline $22 / 06 / 1977$ & Tonga Islands & 61.3 & 8.0 & 7 & 0 & 0 & * \\
\hline $04 / 03 / 1977$ & Romania & 83.6 & 7.5 & 5 & 0 & 0 & * \\
\hline $30 / 11 / 1976$ & Chile-Bolivia & 133.7 & 7.5 & 3 & 0 & 0 & \\
\hline $16 / 08 / 1976$ & Philippines & 33.0 & 8.0 & 3 & 0 & 0 & \\
\hline
\end{tabular}


Table 2.2: List of seismic networks we use in our measurements. Also listed, how many stations we select from the availability of each network.

\begin{tabular}{rrrl}
\hline Network & Total & Selected & Name of \\
Code & Stations & Stations & network \\
\hline BK & 100 & 7 & Berkeley Digital Seismograph Network \\
CD & 11 & 11 & China Digital Seismograph Network \\
CI & 528 & 54 & Caltech Regional Seismic Network \\
CZ & 18 & 1 & Czech Regional Seismic Network \\
EI & 6 & 1 & Irish National Seismic Network (INSN) \\
G & 52 & 34 & GEOSCOPE \\
GE & 142 & 84 & GEOFON \\
GT & 15 & 10 & Global Telemetered Seismograph Network (USAF/USGS) \\
IC & 30 & 30 & New China Digital Seismograph Network \\
I & 54 & 45 & Global Seismograph Network (GSN - IRIS/IDA) \\
IU & 270 & 243 & Global Seismograph Network (GSN - IRIS/USGS) \\
LD & 77 & 1 & Lamont-Doherty Cooperative Seismographic Network \\
LI & 2 & 1 & Laser Interferometer Gravitational-Wave Experiment (LIGO) \\
MN & 29 & 22 & MEDNET Project \\
MS & 6 & 1 & IRIS/Singapore, Singapore National Network (GSN) \\
NZ & 11 & 1 & New Zealand National Seismograph Network \\
OV & 93 & 1 & Observatorio Vulcanològico y Sismòlgico de Costa Rica \\
PL & 8 & 3 & Polish Seismological Network \\
TS & 23 & 16 & TERRAscope (Southern California Seismic Network) \\
TW & 24 & 1 & Broadband Array in Taiwan for Seismology \\
US & 84 & 1 & United States National Seismic Network \\
WM & 4 & 2 & Western Mediterranean Seismic Network \\
ZJ & 15 & 1 & Trans-antarctic Mountains Northern Network \\
\hline
\end{tabular}




\section{A Appendix: Exact momentum equation}

To solve the differential equation, we first multiply Eq. 2.8 by $\mathbf{P}^{-1}$ and obtain

$$
\mathbf{P}^{-1} \mathbf{V} \mathbf{s}+\mathbf{P}^{-1} \mathbf{W} \frac{\partial}{\partial t} \mathbf{s}+\frac{\partial^{2}}{\partial t^{2}} \mathbf{s}=\mathbf{P}^{-1} \mathbf{S}
$$

Because s can be expanded into a set of basis functions (Woodhouse, 1983), we can rewrite the equation in matrix form by defining $\mathbf{b}=\left(\begin{array}{ll}\mathbf{s} & \partial_{t} \mathbf{s}\end{array}\right)^{T}$, and transform Eq. 2.A.35 in a set of two first-order differential equations

$$
\begin{gathered}
\partial_{t}\left(\begin{array}{c}
\mathbf{s} \\
\partial_{t} \mathbf{s}
\end{array}\right)=\left(\begin{array}{cc}
\mathbf{0} & \mathbf{I} \\
-\mathbf{P}^{-1} \mathbf{V} & -\mathbf{P}^{-1} \mathbf{W}
\end{array}\right)\left(\begin{array}{c}
\mathbf{s} \\
\partial_{t} \mathbf{s}
\end{array}\right)+\left(\begin{array}{c}
\mathbf{0} \\
\mathbf{P}^{-1} \mathbf{S}
\end{array}\right) \\
\partial_{t} \mathbf{b}=\mathbf{B} \mathbf{b}+\mathbf{g}
\end{gathered}
$$

We write Eq. 2.A.36 in its compact form in Eq. 2.A.37, where $\mathbf{b}$ is the solution vector, $\mathbf{B}$ is the matrix containing all coupling elements, and $\mathbf{g}$ is the source vector described by a step function and the moment tensor solution. We can find the solution to the homogeneous equation by setting $\mathbf{g}=\mathbf{0}$ and substituting $\mathbf{b}$ by the homogeneous solution $\mathbf{K}(t)$, in order to determine the coefficients of the solution

$$
\partial_{t} \mathbf{K}(t)=\mathbf{B K}(t), \quad \text { where } \quad \mathbf{K}(t)=e^{\mathbf{B} t}
$$

We calculate the non-homogeneous solution by making the source vector $\mathbf{S}$ non-zero and using the particular solution $\mathbf{b}(t)=c(t) \mathbf{K}(t)$. But first, we calculate $c(t)$ by inserting $\partial_{t} \mathbf{b}=\partial_{t} c \mathbf{K}+c \partial_{t} \mathbf{K}$ in Eq. 2.A.37, where we omit $(t)$ to simplify the notation

$$
\begin{aligned}
& { }_{t} c \mathbf{K}+c \partial_{t} \mathbf{K}=c \mathbf{B} \mathbf{K}+\mathbf{g}, \quad \text { where } \partial_{t} \mathbf{K}=\mathbf{B K} \\
& \partial_{t} c \mathbf{K}+c \partial_{t} \mathbf{K}=c \partial_{t} \mathbf{K}+\mathbf{g} \\
& \partial_{t} c \mathbf{K}=\mathbf{g} \\
& \partial_{t} c=\mathbf{K}^{-1} \mathbf{g} \\
& c=\int \mathbf{K}^{-1} \mathbf{g} \mathrm{d} t, \quad \text { integrating by parts } \\
& c=-\mathbf{B}^{-1} \mathbf{K}^{-1} \mathbf{g}+\mathbf{B}^{-1} \mathbf{g}, \quad \text { where } \partial_{t} \mathbf{g}=\mathbf{g} \delta(t) \\
& c=\mathbf{B}^{-1}\left[-\mathbf{K}^{-1}+\mathbf{I}\right] \mathbf{g}
\end{aligned}
$$

Now we substitute $c(t)$ into the particular solution $\mathbf{b}(t)=\mathbf{K}(t) c(t)$ and obtain

$$
\begin{aligned}
& \mathbf{b}(t)=\mathbf{B}^{-1}\left[-\mathbf{K}^{-1}+\mathbf{I}\right] \mathbf{g} \mathbf{K} \\
& \mathbf{b}(t)=\mathbf{B}^{-1}[-\mathbf{I}+\mathbf{K}] \mathbf{g}
\end{aligned}
$$


Finally, we calculate the synthetic seismogram $u$ using the general solution in Eq. 2.A.40, by computing $u=\mathbf{r} \partial_{t}^{2} \mathbf{S}=\mathbf{r} \partial_{t}^{2} \mathbf{b}$, where $\mathbf{r}$ is the receiver vector

$$
\partial_{t}^{2} \mathbf{b}=\mathbf{B} \mathbf{K} \mathbf{g}, \quad \text { and obtain } \quad u=\tilde{\mathbf{r}} \mathbf{B} \mathbf{K} \mathbf{g}=\tilde{\mathbf{r}} \mathbf{B} e^{\mathbf{B} t} \mathbf{g}
$$

$\tilde{\mathbf{r}}$ is the redefined receiver vector $\tilde{\mathbf{r}}=\left(\begin{array}{ll}\mathbf{r} & \mathbf{0}\end{array}\right)^{T}$. Next, we diagonalise matrix $\mathbf{B}$ as $\mathbf{B U}=\mathbf{U} \boldsymbol{\Lambda}$, where $\mathbf{U}$ contains the eigenvectors and $\boldsymbol{\Lambda}$ is the diagonal matrix containing all the eigenvalues of $\mathbf{B}$. We can rewrite the seismogram by substituting $\mathbf{B}=\mathbf{U} \boldsymbol{\Lambda} \mathbf{U}^{-1}$ and $\mathbf{K}(t)=e^{\mathbf{i} \omega t}$, following the form of a harmonic function of time (see Eq. 2.9)

$$
u(t)=\tilde{\mathbf{r}} \mathbf{B} \mathbf{U} e^{\boldsymbol{\Lambda} t} \mathbf{U}^{-1} \mathbf{g}
$$




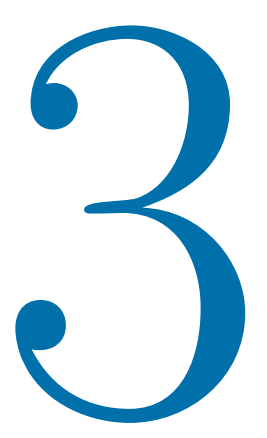

\section{Constraining 1D inner core attenuation using strongly coupled modes}

We measured inner core sensitive normal mode pair ${ }_{10} S_{2-11} S_{2}$, which cross-couples strongly for attenuation and is sensitive to shear wave velocity. We find that our measurements agree with a strongly attenuating inner core. In the past, this mode pair has been used to try to resolve the debate on whether the inner core is strongly or weakly attenuating. Its large spectral amplitude in observed data, possible through the apparent low attenuation of ${ }_{10} S_{2}$, has been explained as evidence of a weakly attenuating inner core. However, this contradicted body waves and other normal modes studies, which resulted in this pair of modes being excluded from inner core modelling. Modes ${ }_{10} S_{2}$ and ${ }_{11} S_{2}$ are difficult to measure and interpret because they depend strongly on the underlying 1D model used. This strong dependence makes these modes change both their oscillation characteristics and attenuation values under a small $1 \mathrm{D}$ perturbation to the inner core model. Here, we include this effect by allowing the pair of modes to cross-couple or resonate through attenuation and treat them as one hybrid mode. We find that, unlike previously thought, the source of ${ }_{10} S_{2}$ visibility is its strong cross-coupling to ${ }_{11} S_{2}$ for both $1 \mathrm{D}$ elastic and anelastic structure. We also observe that the required 1D perturbation is much smaller than the $2 \% v_{s}$ perturbation previously suggested (Andrews et al., 2006), because we simultaneously measure $3 \mathrm{D}$ structure in addition to $1 \mathrm{D}$ structure. Only a $0.5 \%$ increase in inner core $v_{s}$ or a $0.5 \%$ decrease in inner core radius is required to explain ${ }_{10} S_{2-11} S_{2}$ observations and a weakly attenuating inner core is not needed. In addition, the $3 \mathrm{D}$ structure measurements of mode ${ }_{10} S_{2}$ and its cross-coupling to ${ }_{11} S_{2}$ show the typical strong zonal splitting pattern attributed to inner core cylindrical anisotropy, allowing us to add further constrains to deeper regions of the inner core.

The content of this chapter was published as: S. Talavera-Soza and A. Deuss (2020). Constraining 1$\mathrm{D}$ inner core attenuation through measurements of strongly coupled normal mode pairs. Geophysical Journal International 223.1, pp. 612-621. In order to avoid confusion, in this thesis we will refer to the $1 D Q$ cross-coupling explained in Talavera-Soza and Deuss (2020) as attenuation cross-coupling, as it was defined in Andrews et al. (2006). However, both cross-coupling definitions refer to and are calculated in the same manner in both papers. 


\subsection{Introduction}

Inner core solidity was first observed using whole Earth oscillations or normal modes by Dziewonski and Gilbert (1971), although it had already been predicted by a number of authors (i.e. Lehmann, 1936; Birch, 1940; Bullen, 1946) before any direct observations were available. Additional evidence came some three decades later from inner core shear wave measurements, or PKJKP (Deuss et al., 2000; Cao et al., 2005; Wookey and Helffrich, 2008; Tkalčić and Pham, 2018). Currently, inner core solidity together with its shear wave velocity of $v_{s} \sim 3.6 \mathrm{~km} / \mathrm{s}$, are widely accepted.

However, inner core intrinsic attenuation, or absorption of energy as waves travel through Earth, still remains an outstanding problem. The main issue is the incompatibility between body waves studies of compressional wave attenuation $Q_{\alpha}$ and normal mode based studies of shear attenuation $Q_{\mu}$. Body wave studies generally assume the relationship (Anderson and Hart, 1978)

$$
Q_{\alpha}^{-1}=\frac{4}{3}\left(\frac{v_{s}}{v_{p}}\right)^{2} Q_{\mu}^{-1}+\left[1-\frac{4}{3}\left(\frac{v_{s}}{v_{p}}\right)^{2}\right] Q_{\kappa}^{-1}
$$

Using this equation and assuming negligible bulk attenuation $Q_{\kappa}$ together with a Poisson $\operatorname{solid}^{1}\left(v_{s}=\sqrt{3} v_{p}\right)$, we obtain the commonly used conversion relationship $Q_{\alpha}=\frac{9}{4} Q_{\mu}$ (see Anderson and Hart, 1978; Romanowicz and Mitchell, 2015, for details). Using this relationship, compressional body waves show a preference for an even more strongly attenuating inner core in shear than normal modes (e.g. Li and Cormier, 2002).

Early normal modes studies proposed a weakly attenuating inner core with $Q_{\mu}>1000$, based on modes sensitive to $v_{s}$ in the whole inner core, while body waves studies inferred a strongly attenuating inner core with $Q_{\mu} \sim 40$, by assuming no significant bulk attenuation or frequency dependence and mainly constraining the top $500 \mathrm{~km}$ of the inner core (Romanowicz and Mitchell, 2015). The Preliminary Reference Earth Model (PREM, Dziewonski and Anderson, 1981), which was created using a combination of both normal mode and body wave data, has a strongly attenuating inner core with $Q_{\mu}=84$ and has non-zero bulk attenuation with $Q_{\kappa}=1328$ mostly constrained using radial modes. More recent normal modes studies, based on modes sensitive to $v_{p}$ in the shallower and deeper inner core, are also in favour of a strongly attenuating inner core (Widmer-Schnidrig et al., 1991; Durek and Ekström, 1995; Resovsky et al., 2005; de Wit et al., 2014), with $Q_{\mu}=90-110$. Two possibilities have been suggested to overcome the disagreement between body waves and normal modes either (i) non-zero inner core bulk attenuation $Q_{\kappa}$ in order to explain strong compressional wave attenuation, or (ii) frequency dependent $Q_{\mu}$ in order to explain stronger attenuation at high body wave frequencies than at low normal mode frequencies. Unfortunately, no consensus has yet been reached, as even the widely used assumption of shear attenuation dominating over bulk attenuation

${ }^{1} \mathrm{~A}$ Poisson solid is an isotropic elastic material for which the Lamé elastic constants, which arise from the strain-stress relationship, are equal. It has a positive Poisson's ratio. 
a) 1D Coupling synthetics $M_{w} 8.3$ Okhotsk, 2013 PAYG station, $12-60 \mathrm{~h}$

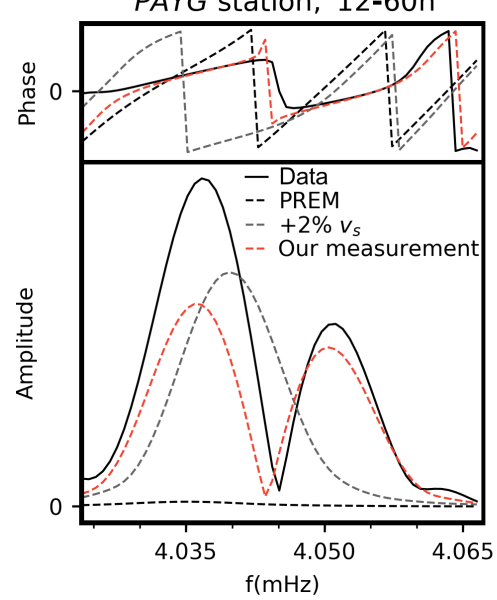

b) 1D Coupling synthetics $M_{w} 8.3$ Okhotsk, 2013 KBS station, $12-60 \mathrm{~h}$

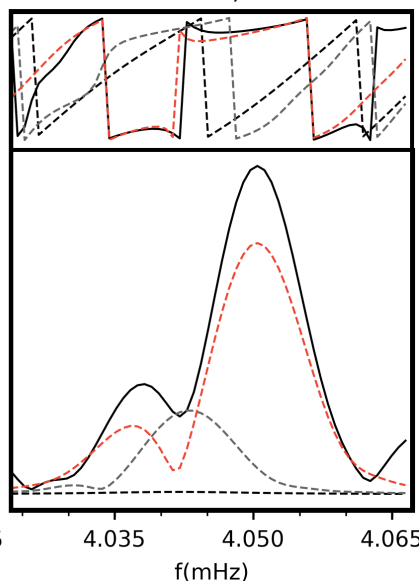

c) Real Spectra data

$M_{w} 8.2$ Bolivia, 1994 All stations, 12-60h

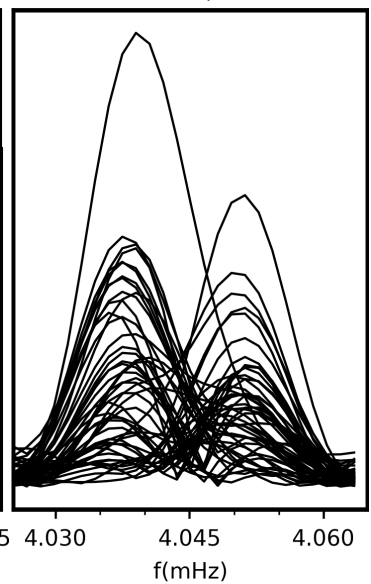

Figure 3.1: Observed data (solid black line) and synthetics for ${ }_{10} S_{2-11} S_{2}$ : (a) station PAYG located in the Galapagos Islands, and (b) station KBS located in Svalbard, Norway. Both stations are from the 2013 Okhotsk event. Synthetics are calculated for PREM (black dashed line), a $2 \%$ inner core $v_{s}$ increase (grey dashed line) and using our measurements of their splitting function coefficients (red dashed line). Also shown are (c) all observed spectra used for the 1994 Bolivia event. Although not shown, a $2 \%$ inner core radius decrease has a similar behaviour on the spectra as a $2 \% v_{s}$ increase.

has now been questioned (Morozov, 2015), since it leads to auxetic elastic materials, which have a negative Poisson's ratio ${ }^{2}$ and are extremely rare in nature.

In the past, the mode pair ${ }_{10} S_{2-11} S_{2}$ has been used to try to resolve the debate on inner core attenuation. In PREM, both modes are mainly inner core confined and have such a low $Q$ (i.e. strong attenuation) that their synthetic spectral amplitudes are more than 100 times smaller than observed earthquake data, rendering them invisible in synthetic normal mode spectra. This does not agree with the real data, where a large peak is visible (see Fig. 3.1). Modes ${ }_{10} S_{2}$ and ${ }_{11} S_{2}$ depend strongly on the underlying 1D model used, changing their oscillation characteristics under small changes to inner core 1D structure (Andrews et al., 2006). Because of this, the discussion about these modes concentrates on what their large amplitudes and their corresponding $Q$ values indicate for inner core attenuation structure.

The centre frequencies $f_{c}$ and $Q$ values, which describe attenuation, of ${ }_{10} S_{2}$ and ${ }_{11} S_{2}$ were first measured by Dziewonski and Gilbert (1973). They identified two peaks in the data and proposed two possible explanations for them: (i) either only ${ }_{10} S_{2}$ is visible and strongly split, or (ii) both modes have visible peaks in the data without significant splitting. They preferred the latter explanation, as at the time

${ }^{2}$ A positive Poisson's ratio signals a material that tends to expand perpendicularly to the direction of compression and vice-versa. 

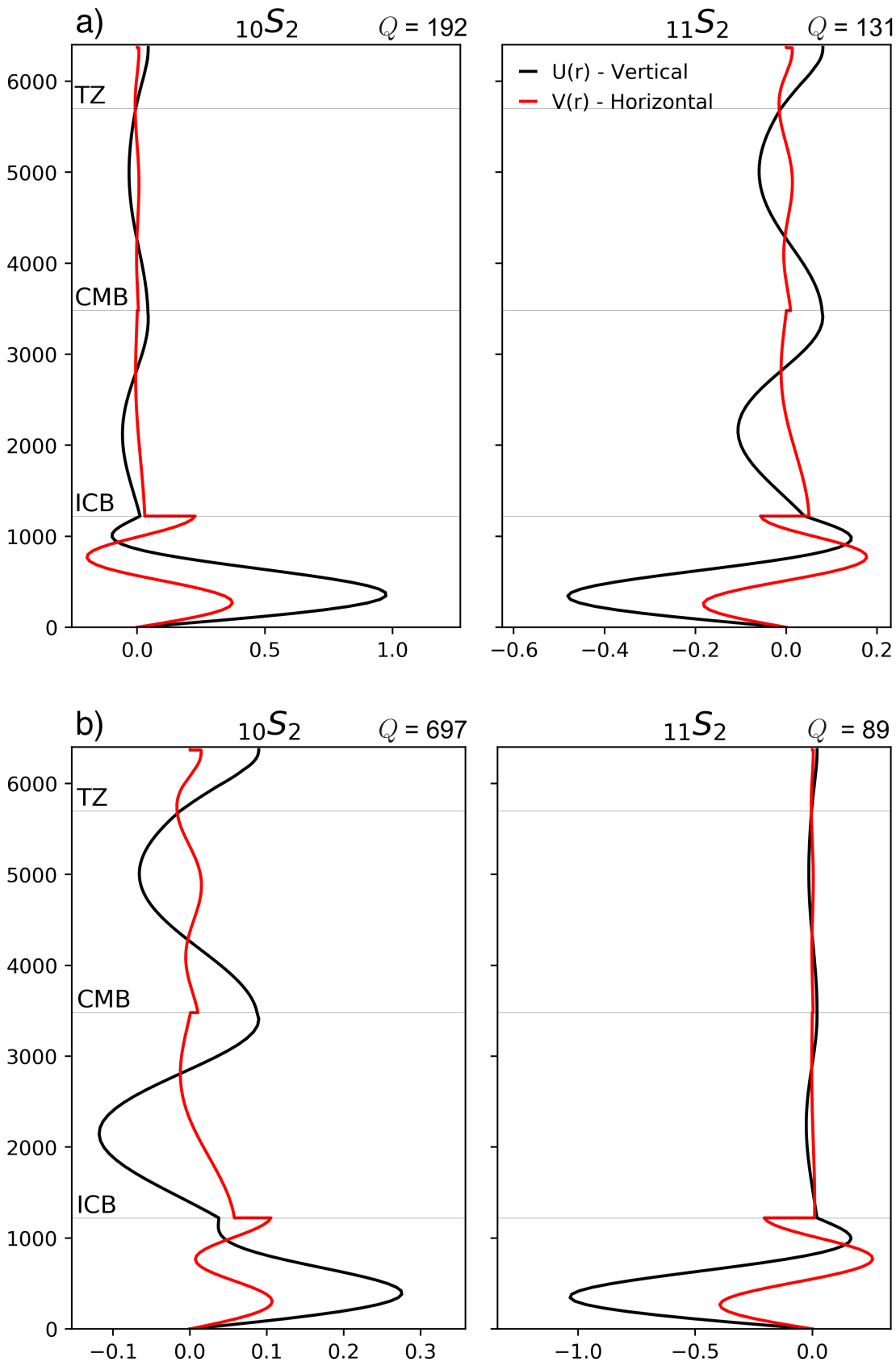

Figure 3.2: Horizontal $V(r)$ and vertical $U(r)$ eigenfunctions and $Q$ values of modes ${ }_{10} S_{2}$ and ${ }_{11} S_{2}$ for (a) PREM and (b) PREM with a $2 \%$ increase in inner core $v_{s}$. Although not shown, a $2 \%$ inner core radius decrease has a similar behaviour on the eigenfunctions and $Q$ values as a $2 \% v_{s}$ increase. 
no other strongly split inner core sensitive modes had been measured. They also concluded that to make ${ }_{11} S_{2}$ visible, the inner core had to be weakly attenuating.

Later, after numerous strongly split inner core sensitive modes were identified, Masters and Gilbert (1981) proposed instead that the source of both peaks was the strong splitting of ${ }_{10} S_{2}$, and interpreted the large amplitude peak of ${ }_{10} S_{2}$ again as proof of a weakly attenuating inner core $\left(Q=1079\right.$ for ${ }_{10} S_{2}$ according to Masters and Gilbert, 1983). This contradicted short period body wave observations, suggesting a frequency dependent $Q_{\mu}$ (Cormier, 1981; Doornbos, 1983; Li and Cormier, 2002). Ritzwoller et al. (1986) confirmed that ${ }_{10} S_{2}$ is strongly split, by comparing nonpolar stations, where they observed two peaks, and polar stations, where they only identified one peak corresponding to ${ }_{10} S_{2}$. Shortly after, Giardini et al. (1988) measured a $Q$ value of 800 for ${ }_{10} S_{2}$, and highlighted the importance of its attenuation cross-coupling to ${ }_{11} S_{2}$ when measuring its splitting, in order to include the strong 1D model dependence of ${ }_{10} S_{2}$ and ${ }_{11} S_{2}$ together with the effects of rotation, ellipticity and 3D structure. Later papers questioned again the observation of ${ }_{11} S_{2}$ and other inner core confined modes (Masters and Shearer, 1990), which prompted the exclusion of these modes in further $Q_{\mu}$ modelling (i.e. Widmer-Schnidrig et al., 1991). As a consequence, modes ${ }_{10} S_{2^{-} 11} S_{2}$ have been excluded in the last few decades from other comprehensive normal mode measurements (i.e. He and Tromp, 1996; Resovsky and Ritzwoller, 1998; Deuss et al., 2013).

More recently, synthetic tests performed by Andrews et al. (2006) confirmed that, in order to take into account the strong $1 \mathrm{D}$ model dependence of ${ }_{10} S_{2-11} S_{2}$, attenuation cross-coupling must be included when using perturbation theory to calculate synthetic spectra. They found that indeed the source of ${ }_{10} S_{2}$ high $Q$ is its strong cross-coupling to ${ }_{11} S_{2}$ for attenuation. Just like Giardini et al. (1988), they point out that in PREM both modes are a mix of PKIKP equivalent mode and inner core confined oscillation (Fig. 3.2a), but that a small perturbation to the $1 \mathrm{D}$ model will change ${ }_{10} S_{2}$ and ${ }_{11} S_{2}$ mode identities (Fig. 3.2b). To include this effect in their synthetics, they combined a $2 \% v_{s}$ perturbation to the inner core with attenuation cross-coupling. Their results had the same effect as recalculating the mode catalogue with a $2 \% v_{s}$ change in the 1D model: one of the modes increased its $Q$ value more than five times to become PKIKP equivalent, while the other mode decreased its $Q$ to become almost completely inner core confined. As a consequence, their synthetic spectra of ${ }_{10} S_{2-11} S_{2}$ now had amplitudes comparable to the real data. The $Q$ values changes in these modes are entirely due to the variations in inner core $v_{s}$, while keeping the inner core strongly attenuating $\left(Q_{\mu}=84\right)$. They showed that their results for ${ }_{10} S_{2}$ and ${ }_{11} S_{2}$ can be obtained using either: (i) perturbation theory, including higher order attenuation cross-coupling together with a 1D perturbation to the 1D underlying model, which is introduced as a non-zero contribution from the $c_{00}$ splitting function coefficient; or (ii) by recalculating the mode catalogue (Woodhouse, 1988; Masters et al., 2011), with both methods giving identical results. While Andrews et al. (2006) only explored the effect of $v_{s}$ on modes ${ }_{10} S_{2-11} S_{2}, 1 \mathrm{D}$ variations to the inner core radius have also been proposed to have a similar effect (Widmer-Schnidrig, 2002). Here, we will explore the effect of both parameters. 
In this chapter, we measure ${ }_{10} S_{2^{-} 11} S_{2}$ and confirm that ${ }_{10} S_{2}$ indeed becomes visible through higher order attenuation cross-coupling. Like in previous studies, we also observe two peaks, which we confirm is due to strong splitting of ${ }_{10} S_{2}$ and is not ${ }_{11} S_{2}$, which we agree is invisible. In addition, we show that the source of the visibility of ${ }_{10} S_{2}$ in real data is indeed due a $1 \mathrm{D}$ perturbation in inner core $v_{s}$ or radius, and not a weakly attenuating inner core. We also apply the same analysis to mode pair ${ }_{8} S_{2-9} S_{2}$ (Fig. 3.A.1), which cross-couples for $v_{p}$ structure. Finally, we use our new measurements for the centre frequency and $Q$ values to determine the amount of change in inner core $v_{s}$ or radius required to explain our observations.

\subsection{Theory}

We concentrate on mode pairs ${ }_{n} S_{l^{-} n^{\prime}} S_{l^{\prime}}$ with $l=l^{\prime}$ and $n=n^{\prime} \pm 1$. These rare occurring pairs are sensitive to $1 \mathrm{D}$ structure (i.e. $s=0$ ), with modes ${ }_{n \pm 1} S_{2}$ ${ }_{n} S_{2}$ coupling for degrees $s=0,2,4$. For some of these mode pairs, degree-zero or attenuation cross-coupling is significantly strong, and by fully including it we obtain the same results using perturbation theory as recalculating the normal mode catalogue. Although counterintuitive, what this so-called attenuation cross-coupling allows us to do is to include the non-linear depth sensitivity of a particular mode to changes in the underlying 1D model, without actually changing the underlying $1 \mathrm{D}$ model while performing our measurements. Instead, the attenuation cross-coupling allows us to use the original eigenfunctions as the basis functions and recombine them into the sensitivity kernels of the changed 1D model. In the cases where the non-linear dependence of the depth sensitivity to the $1 \mathrm{D}$ changes is negligible the effect of including attenuation cross-coupling is also negligible. This means that attenuation cross-coupling acts as a proxy for the strong sensitivity of modes ${ }_{10} S_{2}$ and ${ }_{11} S_{2}$ to small perturbations to $1 \mathrm{D}$ structure, and here we will use the terms interchangeably. For details, see (Andrews et al., 2006).

Here, we exclusively study mode pairs ${ }_{n \pm 1} S_{2^{-}} S_{2}$ that are sensitive to the inner core and cross-couple strongly for attenuation structure (Andrews et al., 2006). The effect of the attenuation cross-coupling is visible when a 1D perturbation to the underlying model is introduced via a non-zero contribution from the $c_{00}$ coefficients. Together with the known effects of rotation and ellipticity, we will measure splitting function coefficients for these modes as a cross-coupled pair, resulting in self-coupled splitting functions for each mode and also their cross-coupled splitting function using the techniques explained in Chapter 2. These pairs become a hybrid multiplet, as their apparent $f_{c}$ and $Q$ values are greatly influenced by the degree-zero elastic and anelastic coefficients of their associated cross-coupled splitting function. In the case where modes cross-couple strongly for attenuation, this effect is excluded when $f_{c}$ and $Q$ are calculated using equations Eq. 2.6 and Eq. 2.7, as both equations were derived using only the self-coupling blocks of the splitting matrix (Giardini et al., 1988). Thus, we report two different $f_{c}$ and $Q$ values for these modes: (i) the $f_{c}$ and $Q$ with respect to PREM, calculated using equations Eq. 2.6 and Eq. 2.7, which can be interpreted with PREM's sensitivity kernels and employed to model inner core structure, and (ii) the hybrid multiplet $f_{c}^{h}$ and $Q^{h}$ values calculated directly from 
their splitting matrix with the diagonal sum rule (Gilbert, 1971), which should not be used to model inner core structure with PREM's sensitivity kernels.

\subsection{Method and Data}

As a large $1 \mathrm{D}$ perturbation is necessary to make modes ${ }_{10} S_{2^{-} 11} S_{2}$ visible in the spectra, we test a range of $c_{00}$ coefficients for the starting model of these modes and their cross-coupling, which encompass any potential 1D perturbation (i.e. $v_{s}$, $v_{p}, \rho$, radius) as seen in Eq. 2.2. The reported uncertainties of the $c_{00}$ coefficients of ${ }_{10} S_{2-11} S_{2}$ were calculated based on the range of starting models that generated similar results.

Modes ${ }_{10} S_{2^{-} 11} S_{2}$ and ${ }_{8} S_{2^{-9}} S_{2}$ are inner core sensitive modes, so we also need to take inner core anisotropy into account (e.g. Woodhouse et al., 1986). Inner core anisotropy with cylindrical symmetry leads to anomalously large values of the zonal coefficients, $c_{20}$ and $c_{40}$. Current inner core anisotropy models over-predict these values or have contradictory predictions for ${ }_{10} S_{2}$ and ${ }_{11} S_{2}$. We try a range of $c_{20}$ values to test the best starting models, as it is usually not possible for inner core sensitive modes to converge to a solution by just starting from PREM, while all our $c_{40}$ coefficients are started from PREM. We also include mantle predictions for comparison to our observations, which are computed using $v_{s}$ model S20RTS (Ritsema et al., 1999), with scaling of the form $\delta v_{p} / v_{p}=0.5 \delta v_{s} / v_{s}$ and $\delta \rho / \rho=0.3 \delta v_{s} / v_{s}$, together with crustal structure model CRUST5.1 (Mooney et al., 1998).

The starting catalogue contains 104 events, but we use a smaller subset which mainly includes selected data from deep earthquakes, because they are better at exciting inner core sensitive modes (Table 4.4).

\subsection{Results}

We have measured splitting function coefficients and corresponding $f_{c}$ and $Q$ values for mode pairs ${ }_{10} S_{2-11} S_{2}$ and ${ }_{8} S_{2-9} S_{2}$, taking all cross-coupling within each mode pair into account. Modes ${ }_{10} S_{2}$ and ${ }_{11} S_{2}$ are strongly dependent on the $1 \mathrm{D}$ model used and are mostly sensitive to $v_{s}$ in the inner core. Their frequencies are very similar in PREM, $4.032 \mathrm{mHz}$ for ${ }_{10} S_{2}$ and $4.058 \mathrm{mHz}$ for ${ }_{11} S_{2}$, making them appear almost on top of each other and therefore cross-coupling strongly for rotation, ellipticity, attenuation and 3D structure. They are isolated from nearby modes and thus, in

Table 3.1: $f_{c}(\mu \mathrm{Hz})$ and $Q$ measurements compared to PREM and apparent $f_{c}^{h}$ and $Q^{h}$ values of the hybrid multiplet, compared to PREM's. Misfits for: PREM $\left(m^{\mathrm{P}}\right)$, S20RTS with CRUST5.1 $\left(\mathrm{m}^{\mathrm{S} 20}\right)$, and our measurements $\left(m^{c_{s t}}\right)$. $N_{e v}$ number of events, $N_{s}$ number of stations used.

\begin{tabular}{rccccccccccc}
\hline & $f_{0}$ & $f_{c}$ & $f_{c}^{h}$ & $Q_{0}$ & $Q$ & $Q^{h}$ & $m^{\mathrm{P}}$ & $m^{\mathrm{S} 20}$ & $m^{c_{s t}}$ & $N_{e v}$ & $N_{s}$ \\
\hline${ }_{8} S_{2}$ & 3214.23 & $3213.83_{-0.02}^{+0.10}$ & 3214.46 & 339 & $270_{-3}^{+5}$ & 267 & \multirow{2}{*}{0.70} & \multirow{2}{*}{0.91} & \multirow{2}{*}{0.34} & \multirow{2}{*}{57} & \multirow{2}{*}{740} \\
${ }_{9} S_{2}$ & 3231.75 & $3230.72_{-0.08}^{+0.18}$ & 3230.78 & 407 & $432_{-5}^{+15}$ & 431 & & & & & \\
\hline${ }_{10} S_{2}$ & 4032.33 & $4043.62_{-2.82}^{+2.82}$ & 4040.09 & 192 & $211_{-1}^{+31}$ & 670 & \multirow{2}{*}{0.97} & \multirow{2}{*}{0.98} & \multirow{2}{*}{0.35} & \multirow{2}{*}{58} & \multirow{2}{*}{1320} \\
${ }_{11} S_{2}$ & 4058.47 & $4069.49_{-2.82}^{+2.82}$ & 4073.11 & 131 & $135_{-5}^{+1}$ & 95 & & & & & \\
\hline
\end{tabular}


this chapter, we will measure them as a pair ignoring further cross-coupling with other modes. PREM also predicts similar sensitivity kernels and $Q$ values, i.e. 192 for ${ }_{10} S_{2}$ and 131 for ${ }_{11} S_{2}$. In PREM, both modes are almost inner core confined and their extremely low $Q$ values make them nearly invisible in its calculated synthetic spectra. However, a small change to PREM's 1D inner core structure can change the modes' oscillation characteristics (Figs. 3.1a-3.1b).

Other mode pairs, of the type ${ }_{n \pm 1} S_{l^{-} n} S_{l}$, have very different $Q$ values, with one mode always being completely inner core confined and with a much lower $Q$ than the other mode. The modes in these other pairs are more separated in frequency and usually overlaid by additional modes, which makes it more complicated to measure their splitting functions. Only ${ }_{8} S_{2}$ and ${ }_{9} S_{2}$ are close enough in frequency to be also measured as a pair. Like ${ }_{10} S_{2^{-} 11} S_{2}$, the pair is sensitive to the inner core and cross-couples for attenuation. Contrary to ${ }_{10} S_{2-11} S_{2},{ }_{8} S_{2-9} S_{2}$ is also sensitive to the mantle and mostly to $v_{p}$ instead of $v_{s}$.

\subsection{1 ${ }_{10} S_{2^{-11}} S_{2}$}

The presence of ${ }_{10} S_{2^{-} 11} S_{2}$ in the real data (Fig. 3.1) provides constraints on inner core shear wave velocity, radius and $Q$. PREM predicts such low $Q$ values for both modes that they are invisible in synthetic spectra when compared to real data. Andrews et al. (2006) showed that mode pair ${ }_{10} S_{2-11} S_{2}$ is strongly dependent on small changes in the underlying 1D model used. Here, these small perturbations to the underlying 1D model are introduced as a non-zero contribution from the $c_{00}$ splitting function coefficient. A peak becomes visible and comparable to the real data only by changing PREM's inner core $v_{s}$ by $2 \%$ and keeping inner core $Q_{\mu}$ at PREM values of 84 (Figs. 3.1a-3.1b). Unlike mode pairs dominated by rotation cross-coupling, which result in the $Q$ values of the two modes to become more similar, a $2 \% v_{s}$ increase leads ${ }_{10} S_{2}$ to become a PKIKP equivalent mode with $Q=706$,

Table 3.2: Modes ${ }_{10} S_{2-11} S_{2}$ predicted splitting function coefficients $\operatorname{Re}\left(c_{00}\right), \operatorname{Im}\left(c_{00}\right), c_{20}$ and $c_{40}$ for inner core and mantle models compared to our measurements, all in $\mu \mathrm{Hz} .\left(^{*}\right)$ Indicates results are not well constrained. Numbers have been rounded-up for an easier comparison. The inner core model predictions shown are as follows: Woodhouse et al. (1986) (Wh), Tromp (1993) (Tr), Durek and Romanowicz (1999) (DR) and Beghein and Trampert (2003) (BT). The predictions of mantle model S20RTS (Ritsema et al., 1999) include the crustal corrections from CRUST5.1 (Mooney et al., 1998).

\begin{tabular}{|c|c|c|c|c|c|c|c|c|c|c|c|}
\hline \multirow[b]{2}{*}{ Predictions } & \multicolumn{3}{|c|}{${ }_{10} S_{2}$} & \multicolumn{4}{|c|}{${ }_{10} S_{2-11} S_{2}$} & \multicolumn{4}{|c|}{${ }_{11} S_{2}$} \\
\hline & $\overline{\operatorname{Re}\left(c_{00}\right) \operatorname{Im}\left(c_{00}\right)}$ & $c_{20}$ & $\overline{c_{40}}$ & $\overline{\operatorname{Re}\left(c_{00}\right) \mathrm{I}}$ & 00) & $c_{20}$ & $\overline{c_{40}}$ & $\operatorname{Re}\left(c_{00}\right) \mathrm{I}$ & $\left.c_{00}\right)$ & $c_{20}$ & $\overline{c_{40}}$ \\
\hline Wh & $-l_{-} \quad-$ & 71 & -44 & - & - & -83 & 53 & - & - & 125 & -67 \\
\hline $\operatorname{Tr}$ & - & 225 & 71 & - & - & -283 & -83 & - & - & 381 & 102 \\
\hline DR & - & 1 & -122 & - & - & -21 & 138 & - & - & 63 & -160 \\
\hline $\mathrm{BT}$ & - & -28 & 23 & - & - & -22 & -25 & - & - & 121 & 24 \\
\hline S20RTS & -3 & 0.3 & -0.2 & -2 & - & 0.2 & -0.2 & -2 & - & 0.2 & -0.1 \\
\hline$+2 \%$ IC $v_{s}$ & 112 & - & - & -142 & - & - & - & 183 & - & - & - \\
\hline$-2 \%$ IC $R$ & 122 & - & - & -148 & - & - & - & 175 & - & - & - \\
\hline Our obs. & 40 & 7 & 16 & -40 & -2 & 5 & 5 & $39^{*}$ & $-1^{*}$ & $8^{*}$ & $-2^{*}$ \\
\hline
\end{tabular}




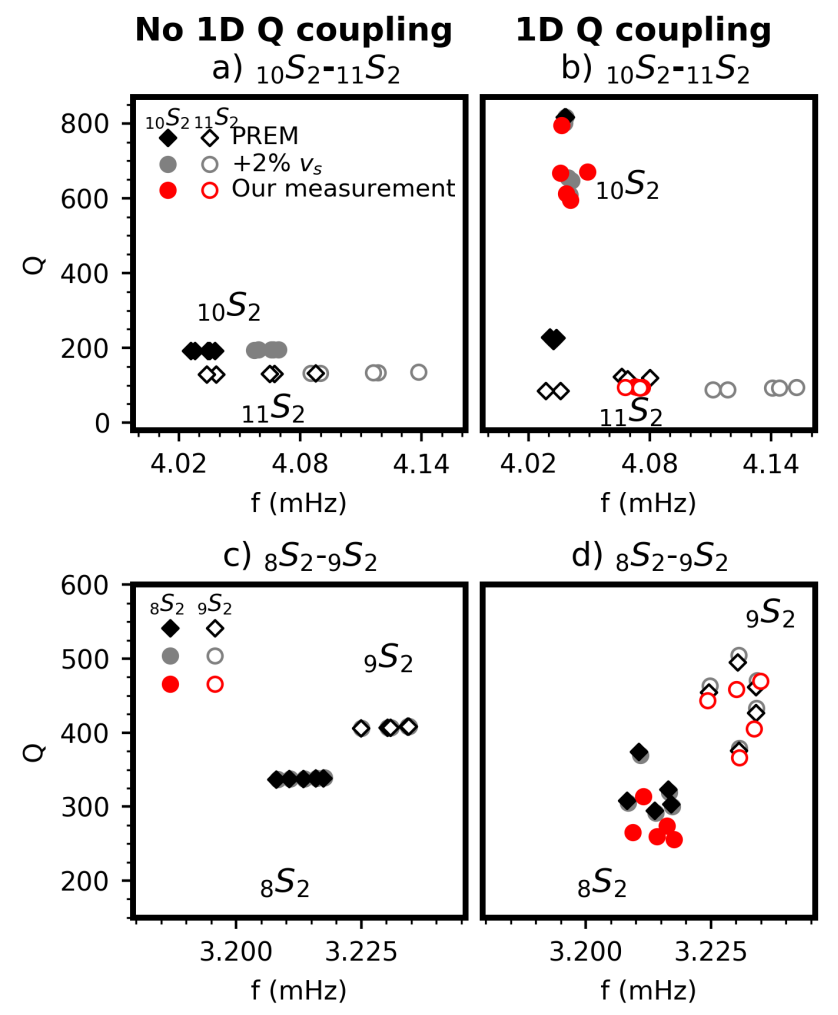

Figure 3.3: Normal mode singlet $Q$ values versus frequencies for: PREM, a $2 \%$ inner core $v_{s}$ increase, and our measurements. All include the effects of rotation and ellipticity, and are calculated using first order perturbation theory. Values are shown for modes ${ }_{10} S_{2-11} S_{2}$ and ${ }_{8} S_{2-9} S_{2}$ : (a, c) without attenuation cross-coupling and (b, d) with attenuation crosscoupling. Although not shown, a $2 \%$ inner core radius decrease has a similar behaviour on the modes singlets as a $2 \% v_{s}$ increase. The measured singlets of ${ }_{10} S_{2}$ and ${ }_{11} S_{2}$ are listed in Table 3.3. See Table 3.A.5 for a comparison between the results obtained using MINEOS (Woodhouse, 1988; Masters et al., 2011) and first order perturbation theory.

sensitive to both the mantle and the inner core, and ${ }_{11} S_{2}$ to become an inner core confined mode with $Q=91$ (Fig. 3.3). The opposite happens when $v_{s}$ decreases by $2 \%$. Similarly, decreasing the inner core radius $R$ by $2 \%$ has the same effect as a $2 \% v_{s}$ increase and vice-versa. The same outcome can be obtained by either recalculating the mode catalogue using MINEOS (Woodhouse, 1988; Masters et al., 2011), or by using perturbation theory including attenuation cross-coupling between these modes (Table 3.A.5). Excluding attenuation cross-coupling prompts the $Q$ of both modes to remain quite similar and close to PREM (Fig. 3.3a), even when including non-zero $c_{00}$ coefficients. For example, if no $v_{s}$ perturbation is present, and only cross-coupling due to rotation and ellipticity is included, then only one of 
Table 3.3: Singlet frequencies and $Q$ values for our measurements of modes ${ }_{10} S_{2}$ and ${ }_{11} S_{2}$ in Fig. 3.3b.

\begin{tabular}{|c|c|c|c|c|c|}
\hline & $f$ & $Q$ & & $f$ & $Q$ \\
\hline \multirow{5}{*}{${ }_{10} S_{2}$} & 4049.05 & 671 & \multirow{5}{*}{${ }_{11} S_{2}$} & 4067.79 & 94 \\
\hline & 4035.81 & 667 & & 4076.15 & 95 \\
\hline & 4036.37 & 794 & & 4074.96 & 93 \\
\hline & 4038.59 & 612 & & 4072.64 & 97 \\
\hline & 4040.56 & 596 & & 4073.98 & 95 \\
\hline
\end{tabular}

the singlets of ${ }_{10} S_{2}$ increases its $Q$ value to 800 (Fig. 3.3b), which is not enough to produce an amplitude peak comparable to the observed spectra (Figs. 3.1a-3.1b).

It is important to note that these changes are due to the $c_{00}$ values varying, which can also be due to changes in $v_{p}$ and density, instead of $v_{s}$ or inner core radius. Thus, we measured ${ }_{10} S_{2^{-}{ }_{11}} S_{2}$ starting from a range of positive and a negative $c_{00}$ values and obtained stable results in both cases. Starting from a positive $c_{00}$ perturbation results in a lower misfit (0.35) and attenuation cross-coupling requires a much smaller deviation from PREM, with ${ }_{10} S_{2}$ becoming the observable mode. Alternatively, starting from a negative $c_{00}$ perturbation leads to a larger misfit (0.46), and the need for a much larger deviation from PREM to make ${ }_{11} S_{2}$ observable. This larger deviation from PREM stems from ${ }_{11} S_{2}$ being predicted to appear further away from the peak seen in the real data. Based on our measurements and synthetics calculations, we conclude that the best possible solution is that starting from a positive $c_{00}$ value for ${ }_{10} S_{2}$ and ${ }_{11} S_{2}$. Positive $c_{00}$ values could be due to either an increase in $v_{s}$ or a decrease in radius; Andrews et al. (2006) arrived to a similar conclusion by comparing their predictions of $f_{c}$ and $Q$ to real data. The added difficulty in measuring these modes stems from ${ }_{11} S_{2}$ becoming an inner core confined mode through its attenuation cross-coupling to ${ }_{10} S_{2}$, making it effectively unobservable and therefore providing insufficient data constraints to make a robust measurement of ${ }_{11} S_{2}$ (Fig. 3.3b). However, we do need to include ${ }_{11} S_{2}$ in our measurements to accurately measure ${ }_{10} S_{2}$ and their cross-coupling. So, mode ${ }_{11} S_{2}$ is measured indirectly by its influence on mode ${ }_{10} S_{2}$ and their cross-coupling. Table 4.4 shows our measured $f_{c}$ and $Q$ values calculated both with respect to PREM and the hybrid multiplet, together with the amount of data and misfits in the inversion.

Mode ${ }_{10} S_{2}$ and its cross-coupling to ${ }_{11} S_{2}$ exhibit anomalous zonal splitting, with strong zonal degree-two $\left(c_{20}\right)$ and degree-four $\left(c_{40}\right)$ values (Table 3.2 ). The typical zonal splitting is visible, with positive splitting anomalies located in the polar areas, and negative values around the equator (Fig. 3.4). The observed zonal splitting cannot be matched by mantle and crustal predictions, and is most easily explained by inner core cylindrical anisotropy with the fast axis aligned with the Earth's rotation axis (e.g. Woodhouse et al., 1986). However, none of the current inner core models can properly explain the observed anomalous splitting, they either over-predict the $c_{20}$ and $c_{40}$ values or even have the opposite expected sign (Table 3.2). ${ }_{10} S_{2}$ is sensitive to the innermost inner core, which is not very well constrained by current anisotropy models. In addition, the fact that we observe anomalous zonal splitting 
a) Our measurement S20+CRUST5.1+IC
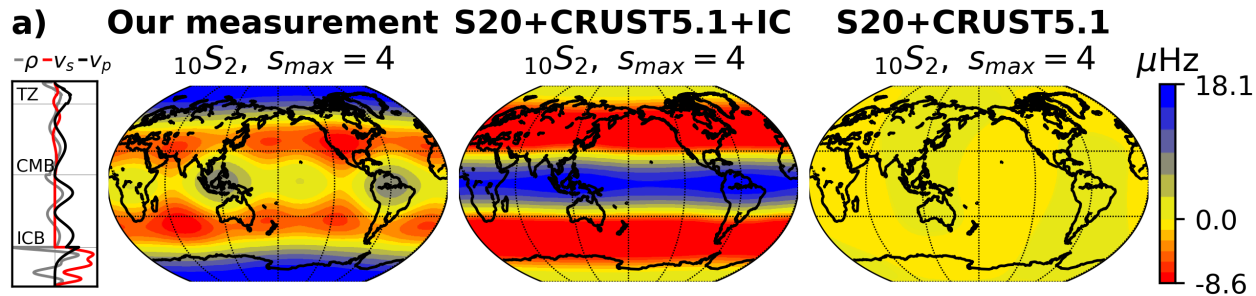

b) ${ }_{10} S_{2-11} S_{2}, \quad s=2-4$
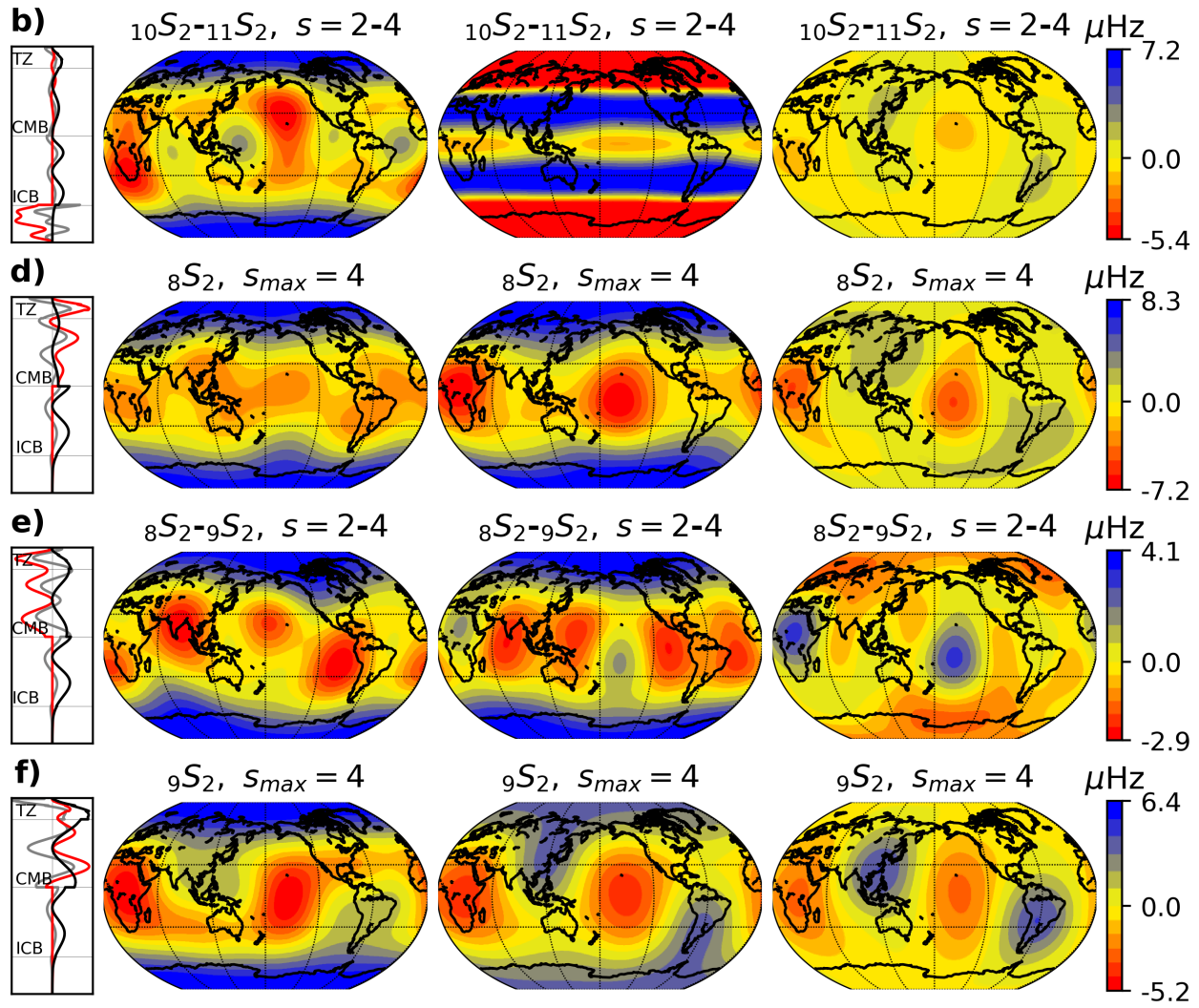

Figure 3.4: Self and cross-coupling splitting functions measurements (left) compared to a combined inner core, mantle and crustal model (middle) and a combined mantle and crustal model (right). Corresponding sensitivity kernels and structural degree $s$ are shown. Inner core model: Beghein and Trampert (2003), mantle model: S20RTS (Ritsema et al., 1999), crustal structure model: CRUST5.1 (Mooney et al., 1998).

in mode ${ }_{10} S_{2}$, and specially in its cross-coupling to ${ }_{11} S_{2}$, shows that cylindrical anisotropy needs to be present in the inner core, as these modes are not dominated by their outer core and mantle sensitivity, even when the $1 \mathrm{D}$ model is perturbed (Fig. 3.2) as we will show in Section 3.5.2. Our ${ }_{10} S_{2-11} S_{2}$ results contradict the suggestion that the origin of the anomalous zonal splitting is as likely to be present in the outer core as in the inner core (Romanowicz and Bréger, 2000). 
Table 3.4: Modes ${ }_{8} S_{2-9} S_{2}$ predicted splitting function coefficients $\operatorname{Re}\left(c_{00}\right), \operatorname{Im}\left(c_{00}\right), c_{20}$ and $c_{40}$ for inner core and mantle models compared to our measurements, all in $\mu \mathrm{Hz}$. Numbers have been rounded-up for an easier comparison. The inner core model predictions shown are as follows: Woodhouse et al. (1986) (Wh), Tromp (1993) (Tr), Durek and Romanowicz (1999) (DR) and Beghein and Trampert (2003) (BT). The predictions of mantle model S20RTS (Ritsema et al., 1999) include the crustal corrections from CRUST5.1 (Mooney et al., 1998).

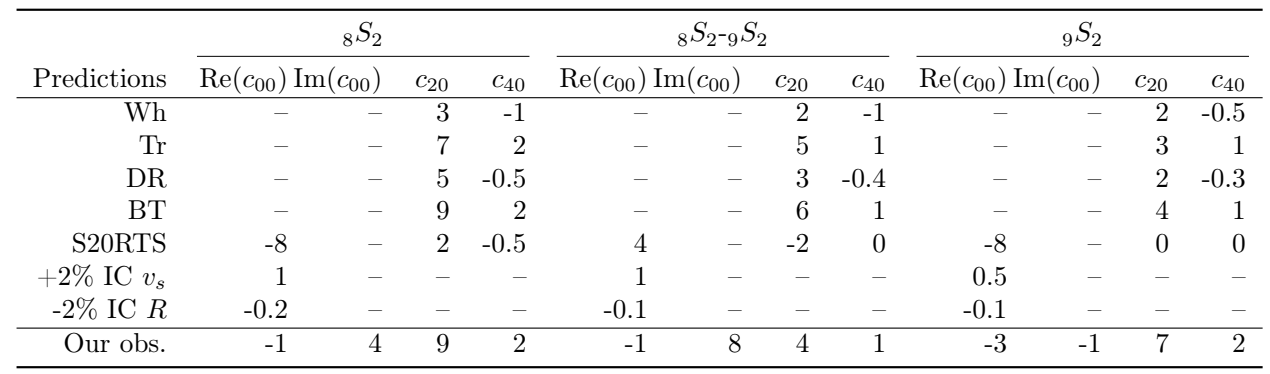

\subsection{2 ${ }_{8} S_{2^{-9}} S_{2}$}

We also measure the mode pair ${ }_{8} S_{2^{-9}} S_{2}$ (Table 4.4). The average $Q$ values of the mode pair ${ }_{8} S_{2-9} S_{2}$ are also affected by attenuation cross-coupling (Figs. 3.3c-3.3d). However, unlike ${ }_{10} S_{2-11} S_{2}$, this mode pair is not significantly affected by a small change in the $1 \mathrm{D}$ model; their radial oscillations are invariant to these changes. Modes ${ }_{8} S_{2-9} S_{2}$ are mostly sensitive to $v_{p}$ (Fig. 3.3d), and our $Q$ value measurements of ${ }_{8} S_{2}$ and ${ }_{9} S_{2}$ are much more similar to PREM predictions.

The splitting functions for modes ${ }_{8} S_{2}$ and ${ }_{9} S_{2}$ and their cross-coupling also show anomalous zonal splitting due to inner core cylindrical anisotropy (Figs. 3.4d-3.4f). These modes agree much better with current inner core model predictions than ${ }_{10} S_{2^{-}}$ ${ }_{11} S_{2}$ (Table 3.4), because they sample the upper part of the inner core (Fig. 3.A.1) and are mainly sensitive to $v_{p}$, which is much better constrained by current inner core anisotropy models.

\subsection{Discussion}

\subsubsection{Inner core radius and shear wave velocity}

We compare our measured $c_{00}$ splitting function coefficients for ${ }_{10} S_{2}$ and ${ }_{11} S_{2}$ with predictions for a range of inner core radius $R$ and shear wave velocity $v_{s}$ variations. We find that both a $2 \%$ decrease in inner core radius $R$ and a $2 \%$ increase in $v_{s}$, like the one suggested by Andrews et al. (2006), are too great (Fig. 3.5). Because we include $3 \mathrm{D}$ variations in our measurements together with $1 \mathrm{D}$ variations, we need a smaller $R$ decrease or $v_{s}$ increase of only around $0.5 \%$. Unlike in the synthetic case, where no $3 \mathrm{D}$ structure variations were included and $1 \mathrm{D}$ structure variations of $2 \%$ were needed to produce similar effects (Fig. 3.3). The $c_{00}$ coefficients of both ${ }_{10} S_{2}$ and the cross-coupling of ${ }_{10} S_{2-11} S_{2}$ fall within a range of $0.5 \%-0.75 \%$ inner core $R$ decrease or $v_{s}$ increase, while the measurement of the less well constrained ${ }_{11} S_{2}$, falls between $0.35 \%-0.5 \% R$ decrease or $v_{s}$ increase. 
To investigate if a small decrease in inner core radius or a small increase in inner core $v_{s}$ would indeed be feasible, we tested the influence of increasing and decreasing $R$ and $v_{s}$ on other inner core sensitive modes. We restrict our comparison to modes dominated by inner core $v_{s}$ using measurements of Deuss et al. (2013). Using perturbation theory, we generate $c_{00}$ predictions by varying PREM's inner core radius $R$ and shear wave velocity $v_{s}$ independently by up-to $1 \%$. We have also applied a mantle and crust correction to the coefficients, using mantle model S20RTS (Ritsema et al., 1999) and crustal model CRUST5.1 (Mooney et al., 1998), as well as rotation and ellipticity corrections. Fig. 3.6 shows that most other $v_{s}$ sensitive modes also agree with either a decrease in inner core radius or an increase in inner core $v_{s}$, in agreement with our observations for ${ }_{10} S_{2^{-} 11} S_{2}$. It is not possible to distinguish between either a change in radius or inner core $v_{s}$, both fit the normal mode frequencies equally well.

Although in theory, our measured $c_{00}$ splitting function coefficients can be due to a perturbation in any of the elastic parameters described in the $1 \mathrm{D}$ model used (Eq. 2.2), we consider the effect of $1 \mathrm{D}$ changes in compressional velocity $v_{p}$ and density $\rho$ negligible. We find that neither an increase nor a decrease in $v_{p}$ can explain mode ${ }_{10} S_{2}$ being visible, and only a disproportionate $40 \%$ increase in inner core density is able to explain it.

If we assume that all contributions to the $c_{00}$ coefficients come from an increase in inner core shear velocity, then our results do not agree with Tkalčić and Pham (2018), who measured an inner core $v_{s} 2.5 \%$ lower than PREM, using body wave observations of inner core shear waves travelling through the whole inner core. We would also disagree with Robson and Romanowicz (2019), who created a model that prefers an average inner core $v_{s} 1 \%$ lower than PREM. In their modelling they allowed $v_{s}, v_{p}$ and $\rho$ to change simultaneously and used 40 spheroidal modes mostly

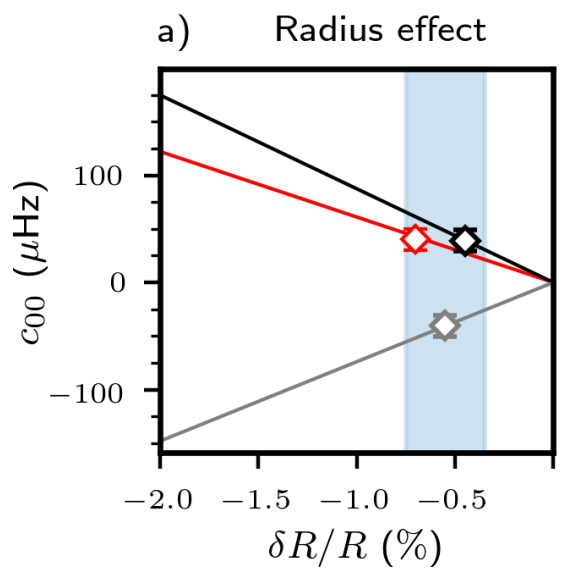

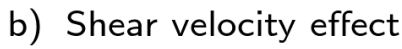

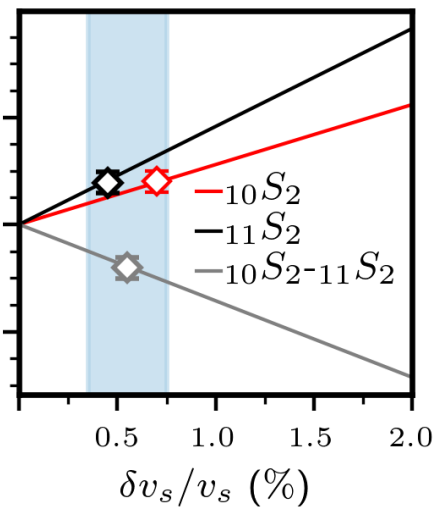

Figure 3.5: $c_{00}$ coefficients as a function of (a) inner core radius $\delta R / R$ and (b) shear wave velocity $\delta v_{s} / v_{s}$, together with our measurements for modes ${ }_{10} S_{2},{ }_{11} S_{2}$ and their crosscoupling. Blue area: (a) $-0.75 \%<\delta R / R<-0.35 \%$ and (b) $0.35 \%<\delta v_{s} / v_{s}<0.75 \%$. 
a) Varying 1D inner core radius $R$

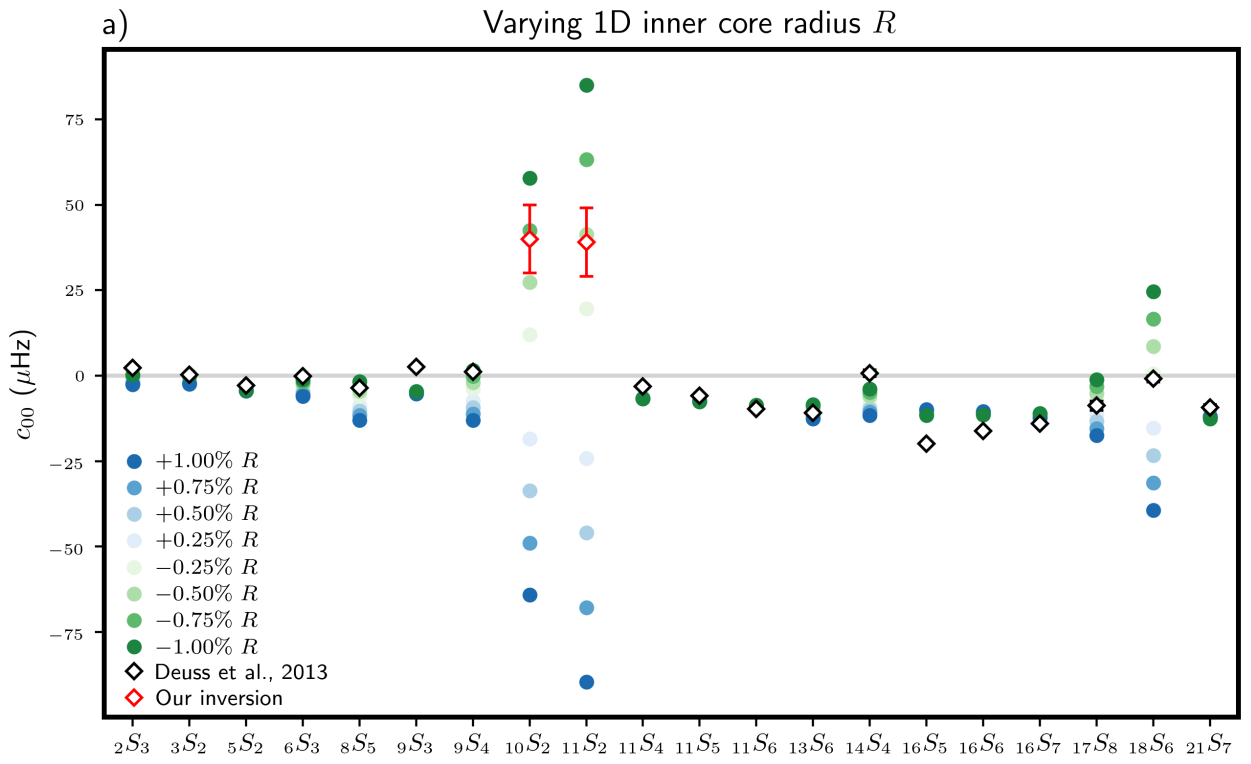

b)

Varying 1D inner core shear velocity $v_{s}$

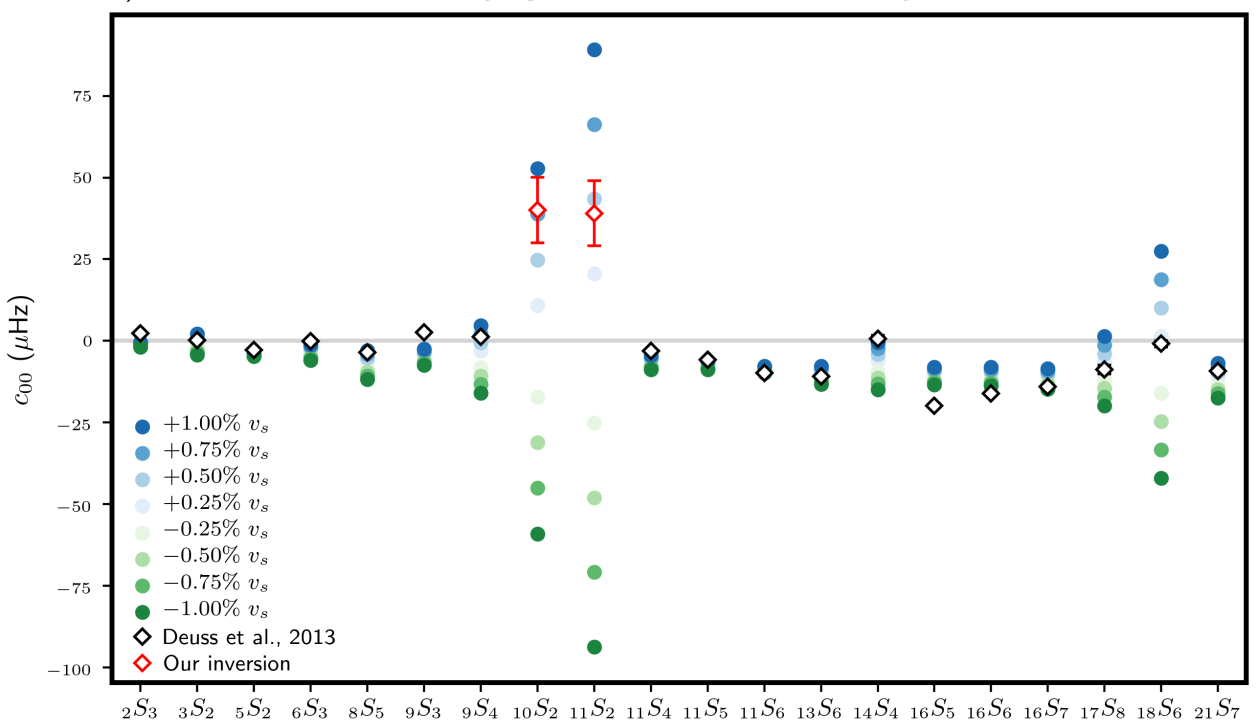

Figure 3.6: Predicted $c_{00}$ coefficients for increasing and decreasing (a) inner core radius $R$ and (b) shear velocity $v_{s}$, compared to Deuss et al. (2013) and our measurements. The coefficients are plotted with respect to PREM (grey line).

sensitive to the shallower inner core. However, we would agree with mineral physics studies of iron at high pressure and temperature, which usually find a $v_{s}$ value $10 \%$ to $20 \%$ larger than PREM (Vočadlo et al., 2003; Vočadlo, 2007). Nonetheless, our proposed increase in $v_{s}$ is still too small to explain the higher values from mineral 
physics. This effect was also explored by Deuss (2008), who showed through a synthetic normal mode study that the large $v_{s}$ found in mineral physics cannot be reconciled with observations of inner core sensitive modes.

On the other hand, if we assume all contributions to the $c_{00}$ coefficients come from a decrease in inner core radius, we would agree with de Wit et al. (2014), who found the inner core radius $0.8 \pm 0.4 \%$ smaller than PREM. Like the studies mentioned in the previous paragraph, they also found the $v_{s}$ of the shallow inner core $\sim 1 \%$ smaller than PREM, although much less constrained than other parameters to be significant. They used 184 spheroidal modes to explore the trade-offs between $v_{p}$, $v_{s}, \rho, Q_{\mu}, Q_{\kappa}$ and depth discontinuities along the whole Earth radius. Although, a $0.5 \%$ inner core radius reduction $(\sim 6 \mathrm{~km})$ seems to reconcile our measurements with previous studies, we should also keep in mind that a simultaneous decrease in inner core $v_{s}$ has also been observed (de Wit et al., 2014; Tkalčić and Pham, 2018; Robson and Romanowicz, 2019). These two effects counteract each other when contributing to the $c_{00}$ coefficients, and can only be properly differentiated in a separate inversion (e.g. de Wit et al., 2014).

Here, for simplicity, we assume that our measured $c_{00}$ values are affected either by inner core radius $R$ or shear wave velocity $v_{s}$ and not a combination. However, to be able to discern between the different contributions of these parameters, our ${ }_{10} S_{2-11} S_{2}$ measurements should be used together with other inner core observations to create a model that is able to explain all of them. This has not been done yet, because mode ${ }_{10} S_{2}$ has long been considered to be an outlier (Durek and Ekström, 1996; Widmer-Schnidrig et al., 1991; Li et al., 1991), both because of its high sensitivity to the underlying 1D model used and the past measurements of its $Q$ value. Here, just like Andrews et al. (2006), we show that the source of ${ }_{10} S_{2^{-1} 11} S_{2}$ behaviour is the result of a perturbation to the $1 \mathrm{D}$ elastic structure of the inner core, and not the $1 \mathrm{D}$ anelastic structure. Thus, the mode pair ${ }_{10} S_{2^{-}{ }_{11}} S_{2}$ can be included in future inner core models.

\subsubsection{Attenuation}

We have shown that the high $Q$ measured for ${ }_{10} S_{2}$ is purely due to its strong attenuation cross-coupling to ${ }_{11} S_{2}$, and not the result of a weakly attenuating inner core. The $Q$ of ${ }_{10} S_{2}$ is strongly dependent on changes to $1 \mathrm{D}$ inner core shear wave velocity $v_{s}$ and radius $R$, as well as cross-coupling due to inner core anisotropy and attenuation. Since attenuation cross-coupling transforms these modes into a hybrid multiplet, we can no longer analyse their $f_{c}$ and $Q$ values with respect to PREM, as their sensitivity kernels and eigenfunctions have also changed. The hybrid multiplet generated has its own apparent $f_{c}^{h}$ and $Q^{h}$, which we interpret as ${ }_{10} S_{2}$ becoming more PKIKP equivalent and ${ }_{11} S_{2}$ more inner core confined. However, these apparent values do not change the underlying $Q_{\mu}=84$ of PREM, and should not be interpreted as evidence of a weakly attenuating inner core. Thus, our ${ }_{10} S_{2^{-} 11} S_{2}$ measurements agree with the strongly attenuating inner core of PREM and suggest that strong inner core attenuation continues from body wave frequencies (e.g. Li and Cormier, 2002), to the lower normal mode frequencies. Moreover, they are now in 
agreement with the other tested $v_{s}$ sensitive inner core modes which also favour a strongly attenuating inner core.

Further tests were run using MINEOS (not shown) on the effect of the outer core's low attenuation on the $f_{c}$ and $Q$ of modes ${ }_{10} S_{2}$ and ${ }_{11} S_{2}$. We find that changing the $Q_{\kappa}$ of the outer core has no significant effect on these modes, which means the resulting $Q$ values must be attributed to the inner core. Unfortunately, we cannot specify a particular inner core depth range for our $Q$ measurements, because of the very nature of splitting functions, which are depth weighted averages of how a mode 'sees' the Earth. We also do not distinguish between the inner core $Q_{\mu}$ and $Q_{\kappa}$ observed by modes ${ }_{10} S_{2}$ and ${ }_{11} S_{2}$ because $Q$ depends on both; although, like previous studies, we do expect both modes to be mostly influenced by $Q_{\mu}$. In order to properly parameterise these variables, an inner core model inversion, that includes our ${ }_{10} S_{2^{-} 11} S_{2}$ measurements and other normal mode data for a much larger number of inner core sensitive modes, should be performed.

In addition, to the ${ }_{10} S_{2-11} S_{2}$ measurements reported in this study, we have also attempted to measure the $3 \mathrm{D}$ elastic and anelastic structure of mode ${ }_{10} S_{2}$ (Mäkinen and Deuss, 2013). For this we performed 4 types of inversions (not shown): (i) measuring elastic and anelastic structure for ${ }_{10} S_{2}$ on its own; (ii) measuring elastic and anelastic structure only for ${ }_{10} S_{2}$ with ${ }_{11} S_{2}$ as predicted by PREM, and including cross-coupling through rotation and ellipticity, but no attenuation cross-coupling; (iii) measuring elastic structure for ${ }_{10} S_{2}$ and ${ }_{11} S_{2}$ and all their cross-coupling, and measuring anelastic structure only for ${ }_{10} S_{2}$; (iv) measuring elastic structure for ${ }_{10} S_{2}$ and ${ }_{11} S_{2}$ and all their cross-coupling, and measuring anelastic structure for ${ }_{10} S_{2}$ and ${ }_{11} S_{2}$. Cases (i) and (ii) produced large misfits $(>0.9)$, even when using the measured elastic and anelastic splitting functions of Pachhai et al. (2020), who measured ${ }_{10} S_{2}$ on its own, as a starting model. Cases (iii) and (iv) give similar results for the center frequency and $Q$ to the ones we obtain without including 3D anelastic structure. Inversions (iii) and (iv) become unstable because of the larger number of parameters that are measured, and we don't consider them well constrained for publication. From these inversions we conclude that attenuation cross-coupling plays an essential role in fitting mode pair ${ }_{10} S_{2^{-} 11} S_{2}$ to the real data. In the future, with the inclusion of additional data, inversions for 3D anelastic inner core splitting functions should be performed in combination with attenuation cross-coupling.

\subsubsection{Innermost inner core anisotropy}

Current inner core models based on normal mode data (Woodhouse et al., 1986; Tromp, 1993; Durek and Romanowicz, 1999; Beghein and Trampert, 2003) predict contradictory $c_{20}$ and $c_{40}$ splitting function values for modes ${ }_{10} S_{2},{ }_{11} S_{2}$ and their cross-coupling (Table 3.2). These models were built using modes mostly sensitive to the uppermost inner core with few exceptions (e.g. ${ }_{3} S_{2}$ ). As a consequence, the seismic anisotropy parameters of these models differ significantly in the innermost $800 \mathrm{~km}$ of the inner core (Irving et al., 2008). Our measurements of ${ }_{10} S_{2}$ and its cross-coupling to ${ }_{11} S_{2}$ (Figs. 3.4a-3.4b) provide new constrains on anisotropy in the innermost inner core, which can be easily included in future inner core anisotropy modelling. 
The predicted $c_{20}$ and $c_{40}$ values of the models are all much larger than our observed values, suggesting that current models overestimate the amount of anisotropy in the innermost inner core. One of the models, Beghein and Trampert (2003), predicts a negative $c_{20}$ value for ${ }_{10} S_{2}$. We find that starting our inversions from negative $c_{20}$ coefficients always leads to higher misfits $(>0.42)$. This implies that our results contradict a flipping in the sign of inner core anisotropy in the lowermost inner core, as the one suggested by Beghein and Trampert (2003). In addition, although all inner core models predict a negative sign for the cross-coupling $c_{20}$ coefficient, we find that the data is best fitted by a positive $c_{20}$, matching the sign of the mantle prediction.

\subsection{Conclusions}

We have measured modes ${ }_{10} S_{2}$ and ${ }_{11} S_{2}$ using perturbation theory including attenuation cross-coupling. We found that ${ }_{10} S_{2}$ is the visible mode and that it is strongly split, while mode ${ }_{11} S_{2}$ becomes completely inner core confined. Our observations are explained by either a $0.5 \%$ increase in inner core $v_{s}$ or a $0.5 \%$ decrease in inner core radius with respect to PREM, and the presence of inner core anisotropy. No change in $Q_{\mu}$ is needed, and our ${ }_{10} S_{2-11} S_{2}$ observations are consistent with a strongly attenuating inner core with PREM values of $Q_{\mu}=84$. Thus, unlike previously thought, a weakly attenuating inner core is not needed to explain observations of ${ }_{10} S_{2^{-}{ }_{11}} S_{2}$, in agreement with past measurements of other inner core sensitive modes. 


\section{A Appendix}

\section{A.1 ${ }_{8} S_{2-9} S_{2}$}
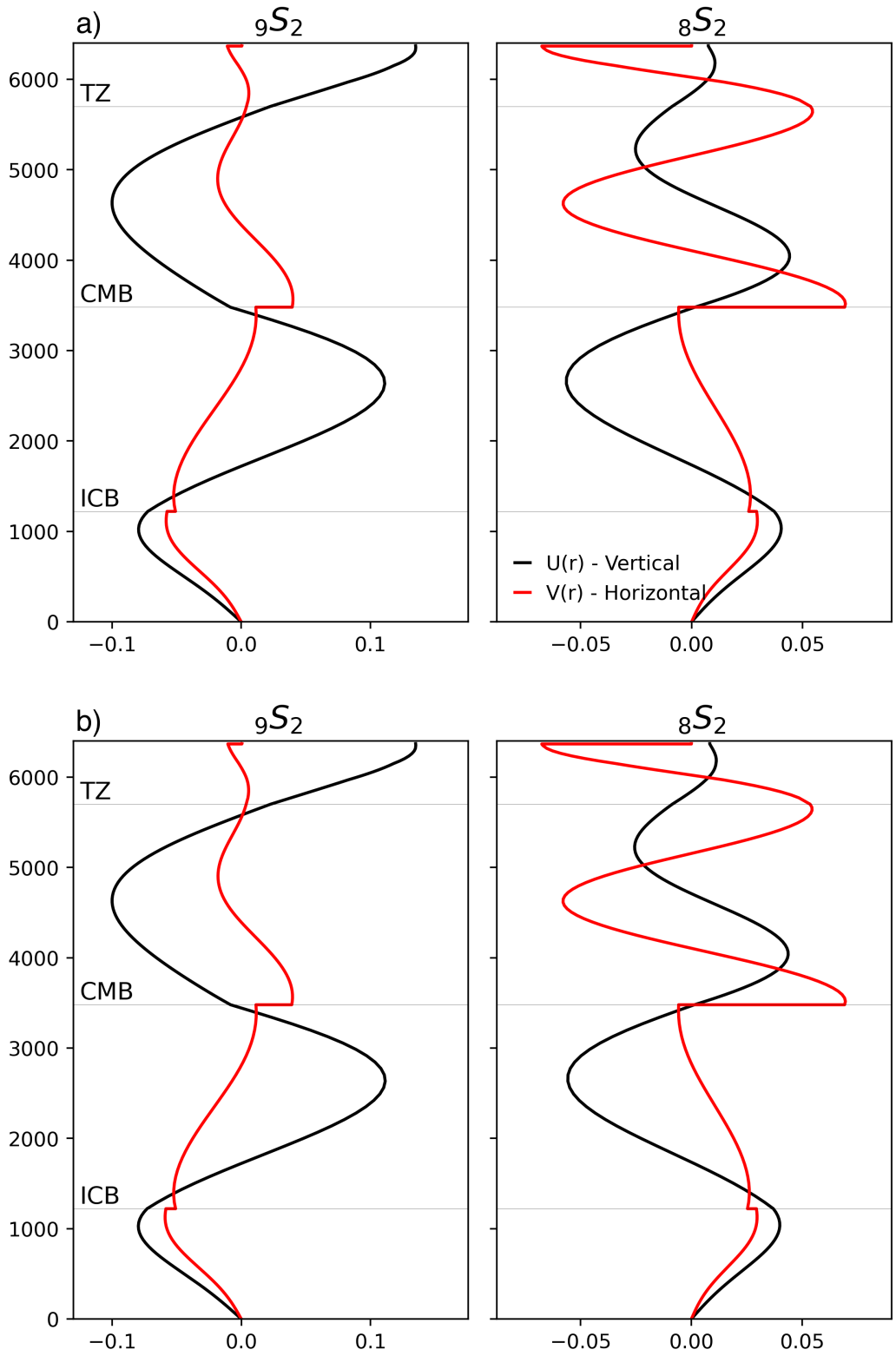

Figure 3.A.1: Horizontal $V(r)$ and vertical $U(r)$ eigenfunctions and $Q$ values of modes ${ }_{9} S_{2}$ and ${ }_{8} S_{2}$ for (a) PREM and (b) PREM with a $2 \%$ increase in inner core $v_{s}$. Although not shown, a $2 \%$ inner core radius decrease has a similar behaviour on the eigenfunctions and $Q$ values as a $2 \% v_{s}$ increase. 


\section{A.2 Comparison between MINEOS and first order perturbation theory}

Table 3.A.5: Center frequencies $f_{c}(\mu \mathrm{Hz})$ and $Q$ values for $2 \%$ perturbation to the inner core radius $R$ and inner core $v_{s}: f_{c}^{\text {MINEOS }}, Q^{\text {MINEOS }}$ obtained by recalculating the mode catalogue using MINEOS (Woodhouse, 1988; Masters et al., 2011); $f_{c}^{\text {per }}, Q^{\text {per }}$ obtained using first order perturbation theory (Chapter 2) together with rotation, ellipticity and attenuation crosscoupling and a small perturbation to the underlying 1D model introduced via a non-zero contribution from the $c_{00}$ coefficients. For comparison $f_{0}$ and $Q_{0}$ are included for PREM (Dziewonski and Anderson, 1981).

\begin{tabular}{|c|c|c|c|c|c|c|c|}
\hline & var. & $f_{0}$ & $f_{c}^{\text {per }}$ & $f_{c}^{\mathrm{MINEOS}}$ & $Q_{0}$ & $Q^{\text {per }}$ & $Q^{\text {MINEOS }}$ \\
\hline \multirow{4}{*}{${ }_{10} S_{2}$} & $+2 \% v_{s}$ & & 4040.70 & 4061.00 & \multirow{4}{*}{192} & 720 & 638 \\
\hline & $-2 \% v_{s}$ & 402232 & 3962.84 & 3967.20 & & 84 & 87 \\
\hline & $+2 \% R$ & 4032.33 & 3960.81 & 3963.82 & & 85 & 88 \\
\hline & $-2 \% R$ & & 4040.85 & 4061.63 & & 763 & 720 \\
\hline \multirow{4}{*}{${ }_{11} S_{2}$} & $+2 \% v_{s}$ & \multirow{4}{*}{4058.47} & 4132.58 & 4130.71 & \multirow{4}{*}{131} & 91 & 89 \\
\hline & $-2 \% v_{s}$ & & 4044.07 & 4064.72 & & 697 & 734 \\
\hline & $+2 \% R$ & & 4045.50 & 4065.85 & & 652 & 695 \\
\hline & $-2 \% R$ & & 4132.99 & 4135.89 & & 90 & 87 \\
\hline
\end{tabular}





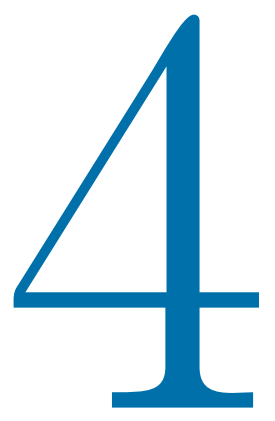

\section{Observing long-period radial modes}

Radial modes, ${ }_{n} S_{0}$, are long-period oscillations that describe the radial expansion and contraction of the whole Earth. They are characterised only by their centre frequency and quality factor $Q$, and provide crucial information about the 1D structure of the Earth. Radial modes were last measured more than a decade ago using only one or two earthquakes. Here, we measure radial modes using 16 of the strongest and deepest earthquakes of the last two decades. By introducing more earthquake data into our measurements, we improve our knowledge of 1D attenuation, as we remove potential earthquake bias from our results. For mode ${ }_{0} S_{0}$, which is dominated by compressional energy, we measure a $Q$ value of 5982 , much higher than previously measured, and requiring less bulk attenuation in the Earth than previously thought. We also show that radial modes cross-couple (resonate) strongly to their nearest spheroidal mode due to ellipticity and inner core cylindrical anisotropy. Cross-coupling improves the fit between data and synthetics, and gives better estimates of the centre frequency and attenuation value of the radial modes. Including cross-coupling in our measurements results in a systematic shift of the centre frequencies of radial modes towards the Preliminary Reference Earth Model (PREM) frequencies. This shift in centre frequencies, has implications for the strength of the radial anisotropy present in the uppermost inner core, with our cross-coupling results agreeing with lower values of anisotropy than the ones inferred from just measuring the modes in self-coupling (isolation). Furthermore, cross-coupling between radial modes and inner core sensitive modes provides constraints on cylindrical inner core anisotropy, that will help us improve our knowledge of the 3D structure of the inner core.

The content of this chapter was published as: S. Talavera-Soza and A. Deuss (2021). New measurements of long period radial modes using large earthquakes. Geophysical Journal International 224.2, pp. $1211-1224$ 


\subsection{Introduction}

Radial modes are long-period oscillations involving the radial expansion and contraction of the whole Earth. They provide crucial information about the 1D structure of the Earth, because they are only described by their centre frequency $f_{c}$ and quality factor $Q$. Radial modes are characterised by their low attenuation and corresponding high $Q$ values (Dziewonski and Gilbert, 1972) and are visible for many days and sometimes even weeks in the spectra of the largest earthquakes. They are dominated by compressional energy, which is dissipated much more slowly than shear energy. Thus, radial modes are especially important in modelling and constraining $1 \mathrm{D}$ bulk attenuation.

Radial mode $Q$ measurements are often combined with other normal modes to make 1D models of attenuation (Sailor and Dziewonski, 1978; Durek and Ekström, 1995; Durek and Ekström, 1996; Widmer-Schnidrig et al., 1991), as well as with surface and body waves (Anderson and Hart, 1978; Dziewonski and Anderson, 1981; Durek and Ekström, 1995; Masters and Widmer-Schnidrig, 1995; Durek and Ekström, 1996; Romanowicz and Durek, 2000; Resovsky et al., 2005). These models mainly constrain shear-wave attenuation, but in order to fit the radial modes the models also require a region with bulk attenuation $Q_{\kappa}$ somewhere in the Earth. Some studies place the region of finite $Q_{\kappa}$ in the upper mantle (Sailor and Dziewonski, 1978) or the lithosphere (Durek and Ekström, 1995), while other studies prefer the inner core (Anderson and Hart, 1978; Dziewonski and Anderson, 1981) or even the outer core or lower mantle (Resovsky et al., 2005). These studies show little agreement on the location of a finite $Q_{\kappa}$ in the Earth and new constraints on radial mode $Q$ values are essential to answer this question.

In the past, only one earthquake, and sometimes even only one station, was used to measure radial modes. All studies published before 1995, used one of the same four large events, and performed stacking and weighting due to the lack of available data (Dratler et al., 1971; Dziewonski and Gilbert, 1972; Dziewonski and Gilbert, 1973; Sailor and Dziewonski, 1978; Buland et al., 1979; Riedesel et al., 1980; Masters and Gilbert, 1983; Park, 1990; Widmer-Schnidrig et al., 1991; Durek and Ekström, 1995; Okal, 1996). He and Tromp (1996) were the first to measure radial modes with more than one event, using the great 1994 Bolivia and Kuril Islands earthquakes. More recently, Okal and Stein (2009), Roult et al. (2010), and Laske and Widmer-Schnidrig (2007) measured the longest period radial modes using the great Sumatra earthquake of 2004. Here, our aim is to improve normal mode measurements by significantly increasing the number of events and include all events of the last 25 years that are large enough to show radial mode observations.

Moreover, we are improving some of the theoretical assumptions that have been made in the past when measuring radial modes. Previous studies measured radial modes using the self-coupling approximation, i.e. in isolation from other modes, which relies on the assumption that radial modes do not resonate with other modes. However, it is well-known that modes, including radial modes, do cross-couple (i.e. resonate) with other modes, in particular when the two modes have similar frequency (e.g. Deuss and Woodhouse, 2001; Irving et al., 2008). Here, we will take cross- 
coupling between radial and nearby modes into account. Prior to our study, Laske et al. (2001) published a brief abstract where they also allow cross-coupling in radial mode measurements. However, they only reported the effect on their centre frequency measurements. They found that when radial modes are allowed to couple, their centre frequencies shift towards values closer to PREM (Dziewonski and Anderson, 1981). They speculated that previous studies may have mistakenly reported measurements of modes coupling to radial modes, as the actual radial mode.

We will show that radial modes may provide us with improved constraints on inner core anisotropy. Radial modes most strongly cross-couple to their closest inner core sensitive spheroidal mode (PKIKP equivalent modes), both through ellipticity and inner core anisotropy (Irving et al., 2008). Cylindrical anisotropy is a wellknown property of the inner core, with the fast axis roughly aligned to the axis of rotation (Woodhouse et al., 1986; Tromp, 1993; He and Tromp, 1996; Durek and Romanowicz, 1999; Beghein and Trampert, 2003; Deuss et al., 2010; Deuss et al., 2013; Mäkinen and Deuss, 2013; Brett and Deuss, 2020). Much less is known about radial anisotropy ${ }^{1}$ in the inner core; only one recent study has shown that the centre frequencies of radial modes may be able to constrain radial inner core anisotropy (Lythgoe and Deuss, 2015).

Here, we will significantly increase the amount of data used to observe radial modes and take cross-coupling to nearby modes into account. We will show that including cross-coupling in our normal mode measurements improves the fit between data and synthetics, and gives better centre frequency and attenuation estimates of radial modes, as well as provides us with new constraints on inner core radial and cylindrical anisotropy.

\subsection{Method}

Radial modes ${ }_{n} S_{0}$, are a special subset of spheroidal modes. These modes have $l=0$ and no nodal lines over the Earth's surface. They describe the radial expansion and contraction of the whole Earth. Here, we will concentrate on measuring radial modes and their coupling to $l=2$ spheroidal modes, ${ }_{n} S_{2}$.

Radial modes with $l=0$ consist of only one singlet and therefore have been considered not to be sensitive to 3D structure. However, this is only true when radial modes are studied using the self-coupling approximation. In self-coupling (SC), a mode is sensitive to structure of degree $s=2 l$, which means radial modes are only sensitive to $s=0$ structure. Radial modes do become sensitive to 3D structure when they are cross-coupled to other modes. In group-coupling (GC), two spheroidal modes with angular orders $l$ and $l^{\prime}$ are sensitive to $\left|l-l^{\prime}\right| \leq s \leq\left|l+l^{\prime}\right|$ structure. Thus, a radial mode ${ }_{n} S_{0}$ will couple with a spheroidal mode ${ }_{n^{\prime}} S_{l^{\prime}}$ for structure with $s=l^{\prime}$. Radial modes most strongly couple with other spheroidal modes with $l^{\prime}=2$, and thus $s=2$ structure, which includes inner core anisotropy, degree-two structure in the mantle and ellipticity. Although these $l^{\prime}=2$ modes can be up-to $0.1 \mathrm{mHz}$ away

${ }^{1}$ Radial anisotropy results in seismic waves travelling at different velocities along the direction of the Earth's radius and perpendicular to it. 
from the radial modes, the strong interactions between them produce wide band crosscoupling, meaning strong coupling between modes that are not so close in frequency.

Here, we make measurements of radial modes in SC and GC, and consider FC a second order effect. We will measure the center frequency and $Q$ values for the radial modes in SC and GC, and the elastic splitting functions for their $l=2$ inner core sensitive coupling pair in SC and GC, using the techniques described in Chapter 2. We include additional $Q$ value uncertainties to radial modes, based on the $Q$ value deviations from the input structure in GC synthetic tests.

\subsection{Data}

Radial modes are most strongly exited by the largest and deepest events and are only slowly attenuating, thus requiring week-long data. Here, we will use 16 of the largest events available in the last 25 years (given in Table 4.1) to invert for radial modes ${ }_{0} S_{0}$ up-to ${ }_{11} S_{0}$, which is a subset of the 104 events described in Section 2.4 and given in Table 2.1. We are including two new events compared to the events used in Deuss et al. (2013) and Koelemeijer et al. (2013), and excluding the 2004 great Sumatra earthquake due to its complicated moment tensor solution. The influence of this earthquake was most notorious for mode ${ }_{0} S_{0}$, with this event requiring a different solution for $Q$ than other earthquakes. Although the effect was minimum for modes with $n>0$, we decided to exclude this earthquake from our final measurements. Our new data set was treated using the same steps as Deuss et al. (2013), and includes

Table 4.1: Earthquakes catalogue for radial modes. The date format is day/month/year, depth is in $\mathrm{km}, M_{w}$ is the moment magnitude as listed in the CMT catalogue and $N_{s}$ is the number of stations available per event.

\begin{tabular}{clrrr}
\hline Date & Location & Depth & $M_{w}$ & $N_{s}$ \\
\hline $30 / 05 / 15$ & Bonin Islands, Japan & 681.0 & 7.9 & 76 \\
$24 / 05 / 13$ & Sea of Okhotsk & 611.0 & 8.3 & 105 \\
$11 / 03 / 11$ & Tohoku, Japan & 29.0 & 9.1 & 139 \\
$27 / 02 / 10$ & Chile & 23.2 & 8.8 & 129 \\
$05 / 07 / 08$ & Sea of Okhotsk & 615.2 & 7.7 & 64 \\
$13 / 01 / 07$ & Kuril Islands & 12.0 & 8.1 & 58 \\
$15 / 11 / 06$ & Kuril Islands & 13.5 & 8.3 & 69 \\
$03 / 05 / 06$ & Tonga Islands & 67.8 & 8.0 & 43 \\
$28 / 03 / 05$ & Northern Sumatra & 25.8 & 8.6 & 91 \\
$23 / 06 / 01$ & Coast of Peru & 29.6 & 8.4 & 88 \\
$25 / 03 / 98$ & Balleny Islands & 28.8 & 8.1 & 66 \\
$17 / 06 / 96$ & Flores Sea & 584.2 & 7.8 & 53 \\
$30 / 07 / 95$ & Northern Chile & 28.7 & 8.0 & 50 \\
$04 / 10 / 94$ & Kuril Islands & 68.2 & 8.3 & 46 \\
$09 / 06 / 94$ & Bolivia & 647.1 & 8.2 & 56 \\
$09 / 03 / 94$ & Fiji Islands & 567.8 & 7.6 & 54 \\
\hline
\end{tabular}


only vertical component data because no high signal-to-noise horizontal component data is available for the radial and spheroidal modes measured in this study.

To select the data's time windows we used each mode's $Q$-cycle as a reference (Dahlen, 1982). The $Q$-cycle is defined as the time necessary for a wave to decay to $e^{-\pi}$ of its original amplitude. A $Q$-cycle of 1.1 was determined as optimal for frequency and $Q$ measurements of modes by Dahlen (1982). This is especially important for radial modes, because if not enough hours are included, we cannot find a solution that satisfies the data at all time windows. A time window end-time significantly lower than the $Q$-cycle will affect the measured center frequency and specially the $Q$ value. Here, we aim to use a $Q$-cycle of 1.1 , however in some cases this was not possible because of noise in the data, and we were limited to a smaller time window. The exact time windows used for every mode are listed in Table 4.2.

In order to invert for the lowest radial order and most weakly attenuating radial modes, the time window was expanded to 83 days for ${ }_{0} S_{0}$ and to 13 days for ${ }_{1} S_{0}$ and ${ }_{2} S_{0}$.

The selection of the radial modes segments was conducted through visual inspection, keeping segments with a high signal-to-noise ratio (ideally above 2 ). We have taken the spectral segments for the $l=2$ spheroidal modes from the extended event catalogue of Deuss et al. (2013) and added spectra for two more recent events. For our GC inversions we used two separate time windows and frequency windows for the spectral segments, one for the radial mode and one for the $l=2$ mode, with the exception of the mode pairs ${ }_{2} S_{0^{-} 7} S_{2}$ and ${ }_{11} S_{0^{-} 27} S_{2}$. The time windows used for the radial modes are listed in Table 4.2, the time windows used for the $l=2$ modes are the ones selected by Deuss et al. (2013). The reasons for using different spectral segments for radial and $l=2$ modes are: (i) the large difference in frequency between the two types of modes (Table 4.2), which are so far away from each other

Table 4.2: Selected time window compared to theoretical $1.1 Q$ cycle for all inverted radial modes, both in hours. Also listed: the $l=2$ mode pair of each radial mode and its sensitivity type, and distance in $\mathrm{mHz}$ between them.

\begin{tabular}{llllll}
\hline $\begin{array}{l}\text { Radial } \\
\text { mode }\end{array}$ & $\begin{array}{l}1.1 Q \\
\text { cycle }\end{array}$ & $\begin{array}{l}\text { time } \\
\text { window }\end{array}$ & $\begin{array}{l}l=2 \\
\text { mode }\end{array}$ & $\begin{array}{l}l=2 \\
\text { type }\end{array}$ & $\begin{array}{l}\text { Distance } \\
\mathrm{mHz}\end{array}$ \\
\hline${ }_{0} S_{0}$ & 1996 & $5-2000$ & ${ }_{2} S_{2}$ & IC & 0.124 \\
${ }_{1} S_{0}$ & 281 & $5-280$ & ${ }_{4} S_{2}$ & $v_{p}$ & 0.091 \\
${ }_{2} S_{0}$ & 151 & $5-150$ & ${ }_{7} S_{2}$ & IC & 0.007 \\
${ }_{3} S_{0}$ & 101 & $35-105$ & ${ }_{8} S_{2},{ }_{9} S_{2}$ & IC & $0.057,0.039$ \\
${ }_{4} S_{0}$ & 72 & $25-100$ & ${ }_{10} S_{2},{ }_{11} S_{2}$ & IC & $0.073,0.047$ \\
${ }_{5} S_{0}$ & 58 & $35-90$ & ${ }_{13} S_{2}$ & IC & 0.039 \\
${ }_{6} S_{0}$ & 49 & $20-75$ & ${ }_{16} S_{2}$ & IC & 0.043 \\
${ }_{7} S_{0}$ & 41 & $10-65$ & ${ }_{18} S_{2}$ & IC & 0.035 \\
${ }_{8} S_{0}$ & 35 & $10-55$ & ${ }_{20} S_{2}$ & IC & 0.067 \\
${ }_{9} S_{0}$ & 31 & $10-50$ & ${ }_{22} S_{2}$ & IC & 0.056 \\
${ }_{11} S_{0}$ & 26 & $8-50$ & ${ }_{27} S_{2}$ & IC & 0.023 \\
\hline
\end{tabular}


in frequency that many other modes lie in between them; (ii) radial modes and $l=2$ modes have different $Q$ cycles, (iii) $l=2$ modes usually lie in the same frequency window as other strongly attenuating modes. Furthermore, using synthetic spectra, we verified that the influence of other modes in between the spectral segments of the radial modes and their closest $l=2$ modes is not significant, and can be disregarded for the purpose of this chapter.

Overall, our new data set has a factor of 10 increase in data size when compared to previous studies (Widmer-Schnidrig et al., 1991; Durek and Ekström, 1995; He and Tromp, 1996). We include between 3 and 14 earthquakes in each of our radial mode measurements (see Table 4.3), whereas studies before only used one or two events.

\subsection{Results}

We first use our new data set to measure radial modes using the self-coupling (SC) approximation, which allows us to compare our measurements to previous studies (see Section 4.4.1). Next, we include group-coupling (GC) to nearby $l=2$ modes to investigate the influence of coupling on the measured centre frequency $f_{c}$ and quality factor $Q$ (see Section 4.4.2). Finally, we interpret our $f_{c}$ measurements to obtain new constraints on inner core anisotropy and 1D bulk attenuation (see Section 4.5).

\subsubsection{Self-coupling approach (SC)}

We first measured radial modes ${ }_{0} S_{0}$ up-to ${ }_{9} S_{0}$ in the self-coupling (SC) approach. ${ }_{10} S_{0}$ was not measured because of its low spectral amplitude and the presence of too many overriding nearby modes. ${ }_{11} S_{0}$ could not be measured using SC, because of its close proximity to mode ${ }_{27} S_{2}$ and its significant amplitude difference with this

Table 4.3: Misfit comparison of the SC and GC approach to previous measurements. Misfits for: PREM $\left(m^{\mathrm{P}}\right)$; REM website $\left(m^{\mathrm{REM}}\right.$, Laske et al., 2005); He and Tromp (1996) $\left(m^{\mathrm{HT}}\right)$; and our measurements $\left(m^{\mathrm{SC}}, m^{\mathrm{GC}}, m^{\mathrm{GC}}\right) \cdot m^{\mathrm{GC}^{e}}$ represents our measurements including cross-coupling only due to ellipticity between radial modes and the $l=2$ modes, while $m^{\mathrm{GC}}$ includes all cross-coupling. The F-test status is shown as the confidence level at which the misfit reduction is significant between $m^{\mathrm{GC}}{ }^{e}$ and $m^{\mathrm{GC}}$. For the cases where $m^{\mathrm{GC}}$ and $m^{\mathrm{GC}^{e}}$ were calculated using the same number of inverted parameters no F-test was calculated. Number of events $N_{e v}$ and number of stations $N_{s}$ are listed both for the radial modes and $l=2$ modes spectral data. All misfit calculations include the effects of rotation and ellipticity.

\begin{tabular}{|c|c|c|c|c|c|c|c|c|c|c|c|c|c|}
\hline & $N_{s}$ & $N_{e v}$ & $m^{\mathrm{P}}$ & $m^{\text {REM }}$ & $m^{\mathrm{HT}}$ & $m^{\mathrm{SC}}$ & $m^{\mathrm{GC}^{e}}$ & $m^{\mathrm{GC}}$ & F-test & & $N_{s}$ & $N_{e v}$ & $m^{\mathrm{GC}}$ \\
\hline${ }_{0} S_{0}$ & 25 & 5 & 1.59 & 2.58 & - & 0.04 & 0.04 & - & - & ${ }_{2} S_{2}$ & - & - & - \\
\hline${ }_{1} S_{0}$ & 224 & 9 & 0.32 & 0.29 & 0.14 & 0.082 & 0.11 & 0.081 & $99 \%$ & ${ }_{4} S_{2}$ & 1537 & 90 & 0.31 \\
\hline${ }_{2} S_{0^{-7} 7} S_{2}$ & 300 & 9 & 2.46 & 0.82 & 2.74 & 0.38 & 0.15 & 0.13 & $99 \%$ & & & & \\
\hline${ }_{3} S_{0}$ & 139 & 5 & 1.59 & 0.45 & 0.45 & 0.44 & 0.45 & 0.37 & $99 \%$ & ${ }_{8} S_{2-9} S_{2}$ & 740 & 57 & 0.34 \\
\hline${ }_{4} S_{0}$ & 428 & 11 & 0.62 & 0.54 & 0.55 & 0.55 & 0.53 & 0.51 & $99 \%$ & ${ }_{10} S_{2-11} S_{2}$ & 1320 & 58 & 0.33 \\
\hline${ }_{5} S_{0}$ & 146 & 5 & 0.93 & 0.32 & 0.46 & 0.23 & 0.208 & 0.205 & $95 \%$ & ${ }_{13} S_{2}$ & 700 & 37 & 0.14 \\
\hline${ }_{6} S_{0}$ & 134 & 5 & 1.65 & 0.17 & 0.23 & 0.16 & 0.16 & - & - & ${ }_{16} S_{2}$ & - & - & - \\
\hline${ }_{7} S_{0}$ & 68 & 3 & 1.90 & 0.56 & - & 0.37 & 0.24 & - & - & ${ }_{18} S_{2}$ & - & - & - \\
\hline${ }_{8} S_{0}$ & 58 & 9 & 1.11 & 0.78 & 0.73 & 0.67 & 0.64 & - & - & ${ }_{20} S_{2}$ & - & - & - \\
\hline${ }_{9} S_{0}$ & 376 & 14 & 1.99 & 1.15 & 0.99 & 0.39 & 0.36 & - & - & ${ }_{22} S_{2}$ & - & - & - \\
\hline${ }_{11} S_{0-27} S_{2}$ & 355 & 23 & 1.72 & - & - & - & - & 0.29 & - & & & & \\
\hline
\end{tabular}




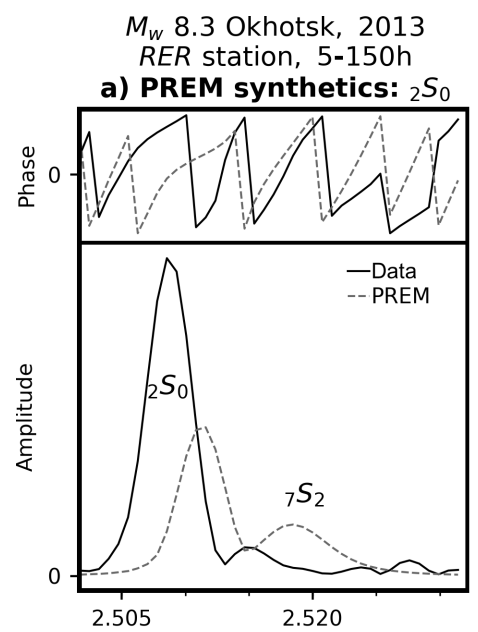

$M_{w} 8.2$ Bolivia, 1994

KEV station, 35-90h

b) PREM synthetics: ${ }_{5} S_{0}$

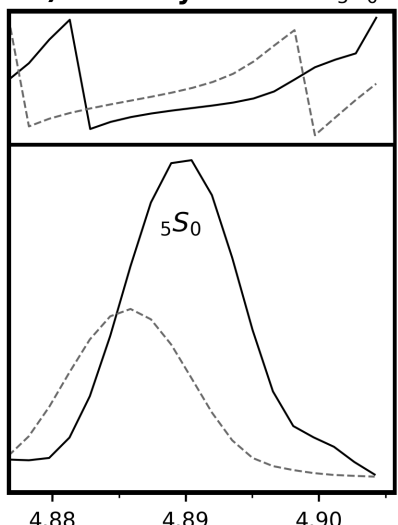

$M_{w} 7.9$ Bonin Islands, 2015

INCN station, 5-50h

c) PREM synthetics: ${ }_{11} S_{0}$

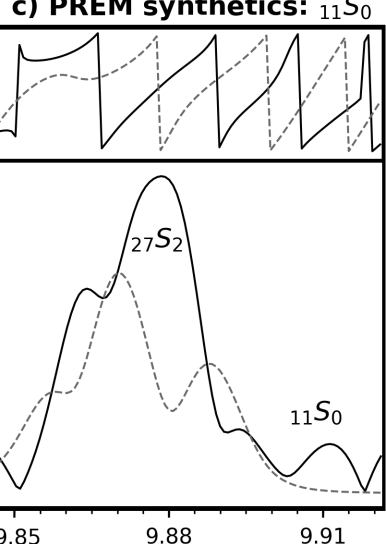

f) Our measurement: ${ }_{11} S_{0}$
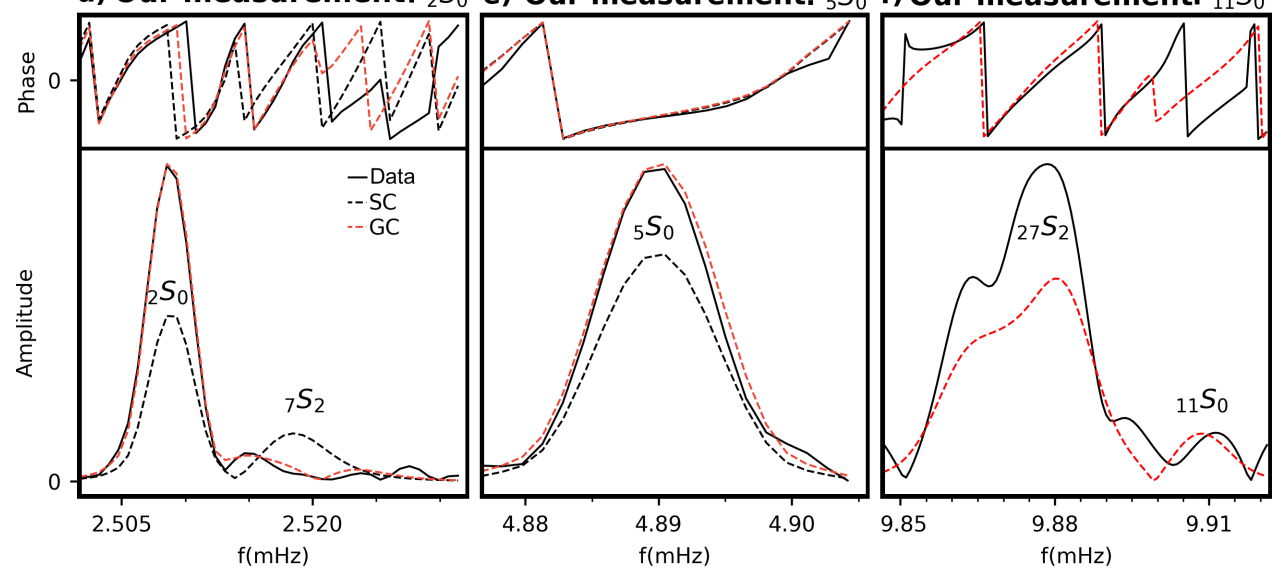

Figure 4.1: Amplitude and phase spectra for radial modes ${ }_{2} S_{0^{-}-7} S_{2},{ }_{5} S_{0}$ and ${ }_{11} S_{0^{-}-27} S_{2}$. Data compared to $(\mathrm{a}, \mathrm{b}, \mathrm{c})$ 1D PREM synthetics together with rotation and ellipticity and no cross-coupling, and (d, e, f) our self-coupling (SC) and group-coupling (GC) measurements. The frequency window showed for ${ }_{2} S_{0-7} S_{2}$ and ${ }_{11} S_{0-27} S_{2}$ is extended beyond our selected frequency window for the inversion. Mode ${ }_{13} S_{2}$ is not shown as it has both a different selected time and frequency window as ${ }_{5} S_{0}$.

mode. For modes ${ }_{0} S_{0},{ }_{2} S_{0},{ }_{5} S_{0},{ }_{7} S_{0},{ }_{8} S_{0},{ }_{9} S_{0}$ and ${ }_{11} S_{0}$, it was necessary to start from a given centre frequency shift, which we determined by visually inspecting the data. This step, which was also necessary when conducting synthetics tests, is needed when PREM's predictions lie too far away from the data (Figs. 4.1a-4.1c), and given that we use only small steps in our iterated non-linear inversion, we need to use a starting model that moves in the right direction. For all other radial modes, inversions were started from PREM. 
Our measured centre frequency and quality factor values are shown in Table 4.4. Fig. 4.1 displays normal mode spectra for radial modes ${ }_{2} S_{0}$ and ${ }_{5} S_{0}$, showing the fit between the observed data, PREM synthetics and synthetics calculated using our $f_{c}^{S C}$ and $Q^{S C}$ values measured in the SC approximation. In these individual spectra, we see that our SC measurements improve the data fit compared to the PREM synthetics. Table 4.3 shows that for our whole data set, the SC misfit is better than the PREM misfit. For comparison, the misfit using centre frequency and quality factor values from previous studies are also included (He and Tromp (1996) and the Reference Earth Model webpage (REM, Laske et al., 2005)). Again, we see that our values have the lowest misfit.

We compare our $f_{c}$ and $Q$ measurements and their uncertainties to PREM (Fig. 4.2), and against the measurements from previous studies (Widmer-Schnidrig et al., 1991; Durek and Ekström, 1995; He and Tromp, 1996; Laske et al., 2005). Our SC results agree with these studies, which only used the SC approach, especially the $f_{c}$ values are very similar. This implies that the $f_{c}$ measurements are very robust, and can be derived even with the limited data used in previous studies. On the other hand, much larger variation is seen in the quality factor $Q$ between the different studies. For modes ${ }_{5} S_{0}$ and ${ }_{9} S_{0}$ we obtain lower $Q$ values. The other radial modes are within the $Q$ error bars of previous measurements. Although the $Q$ measurement of mode ${ }_{0} S_{0}$ falls within the error bars given by previous measurements, we do measure a significantly higher $Q$ value than before.

\subsubsection{Group-coupling approach (GC)}

\subsubsection{Radial Modes}

Next, we include group-coupling (GC) in our measurements. Radial modes have their strongest cross-coupling with $l=2$ inner core sensitive spheroidal modes, with differences in frequency between the cross-coupled modes of up-to $0.124 \mathrm{mHz}$ (Table 4.2). Fig. 4.3 shows the sensitivity kernels as a function of depth for the radial and $l=2$ spheroidal modes. Radial modes (Fig. 4.3a) are mostly sensitive

Table 4.4: Radial modes measurements of centre frequencies $f_{c}(\mu \mathrm{Hz})$ and $Q$ in SC and GC. PREM centre frequency $f_{0}(\mu \mathrm{Hz})$ and quality factor $Q_{0}$ are also given.

\begin{tabular}{rcccccc}
\hline & $f_{0}$ & $f_{c}^{S C}$ & $f_{c}^{G C}$ & $Q_{0}$ & $Q^{S C}$ & $Q^{G C}$ \\
\hline${ }_{0} S_{0}$ & 814.31 & $814.6010_{-0.0014}^{+0.0007}$ & $814.6010_{-0.0014}^{+0.0007}$ & 5327 & $5982_{-174}^{+211}$ & $5982_{-174}^{+211}$ \\
${ }_{1} S_{0}$ & 1631.34 & $1631.55_{-0.01}^{+0.004}$ & $1631.56_{-0.12}^{+0.02}$ & 1499 & $1849_{-20}^{+7}$ & $1856_{-143}^{+144}$ \\
${ }_{2} S_{0}$ & 2510.48 & $2508.45_{-0.08}^{+0.06}$ & $2509.30_{-0.22}^{+0.14}$ & 1242 & $1241_{-68}^{+97}$ & $1788_{-122}^{+117}$ \\
${ }_{3} S_{0}$ & 3271.18 & $3272.40_{-0.04}^{+0.06}$ & $3271.98_{-0.04}^{+0.06}$ & 1083 & $1222_{-22}^{+75}$ & $1242_{-46}^{+80}$ \\
${ }_{4} S_{0}$ & 4105.76 & $4106.51_{-0.12}^{+0.05}$ & $4105.99_{-0.43}^{+0.03}$ & 969 & $1157_{-31}^{+86}$ & $1176_{-33}^{+109}$ \\
${ }_{5} S_{0}$ & 4884.17 & $4888.34_{-0.03}^{+0.04}$ & $4886.72_{-0.16}^{+0.19}$ & 921 & $1016_{-11}^{+31}$ & $1008_{-13}^{+11}$ \\
${ }_{6} S_{0}$ & 5740.25 & $5742.24_{-0.05}^{+0.09}$ & $5742.11_{-0.57}^{+0.007}$ & 913 & $1086_{-32}^{+52}$ & $1077_{-34}^{+111}$ \\
${ }_{7} S_{0}$ & 6580.71 & $6586.58_{-0.54}^{+0.29}$ & $6583.56_{-0.13}^{+0.55}$ & 881 & $1035_{-47}^{+132}$ & $883_{-66}^{+87}$ \\
${ }_{8} S_{0}$ & 7424.13 & $7430.30_{-0.40}^{+0.16}$ & $7429.66_{-0.40}^{+0.14}$ & 852 & $1305_{-223}^{+1}$ & $1198_{-45}^{+139}$ \\
${ }_{9} S_{0}$ & 8262.64 & $8270.01_{-0.11}^{+0.11}$ & $8268.27_{-0.21}^{+0.25}$ & 840 & $933_{-28}^{+51}$ & $965_{-46}^{+63}$ \\
${ }_{11} S_{0}$ & 9865.33 & - & $9893.37_{-0.35}^{+0.004}$ & 832 & - & $1053_{-1}^{+93}$ \\
\hline
\end{tabular}




\section{Radial Modes}

a) Center frequency

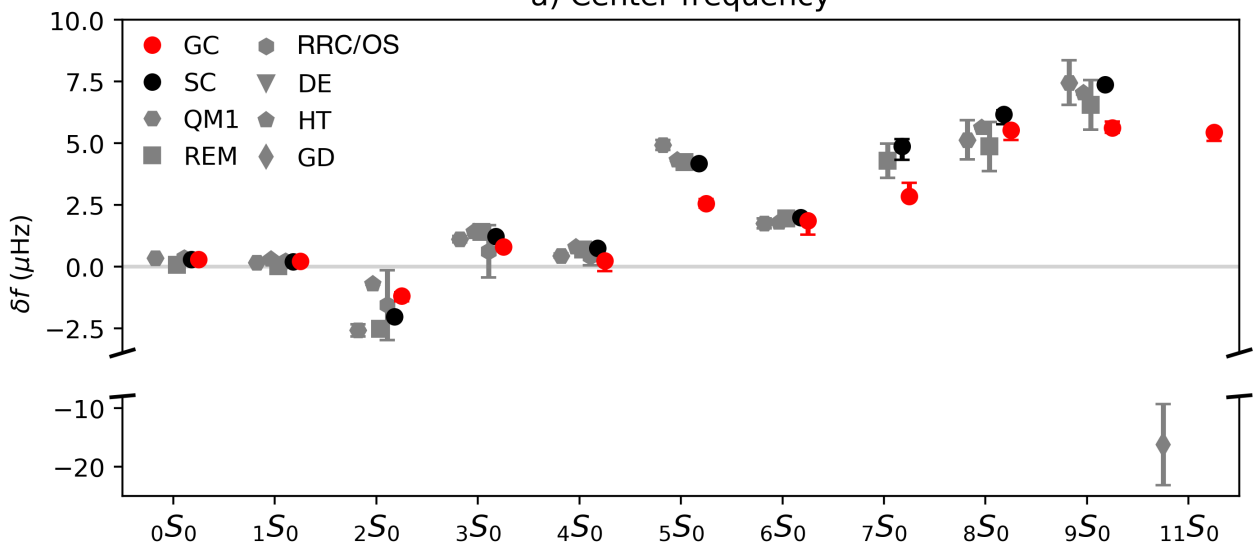

b) Quality factor

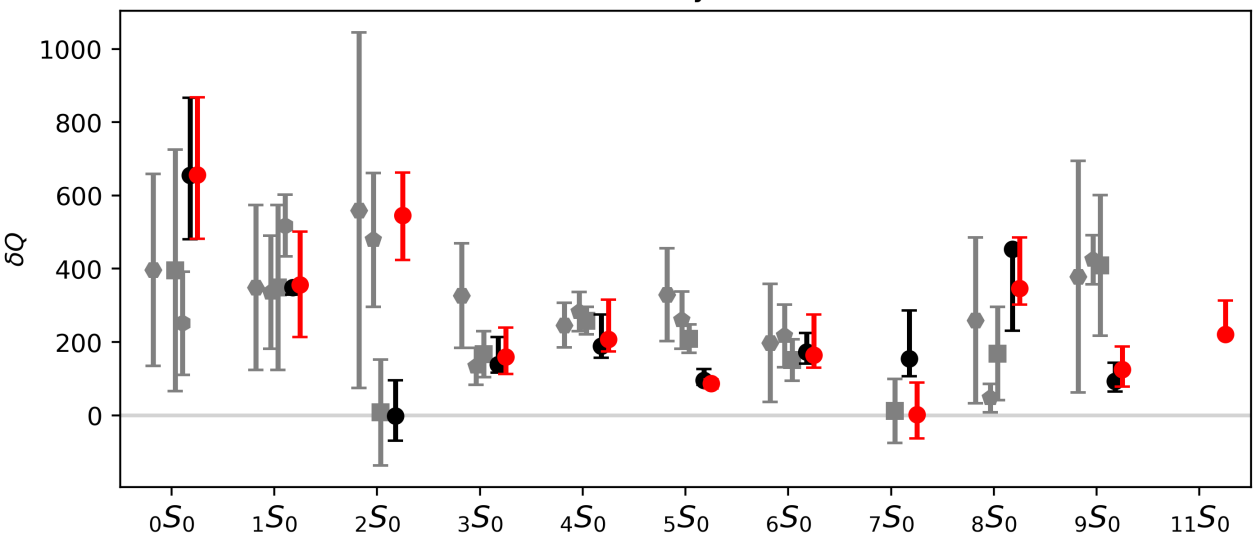

Figure 4.2: Radial modes measurements with respect to PREM. $f_{c}$ and $Q$ shifts are compared to previous observations in grey. SC: our self-coupling measurements (black), GC: our group-coupling measurements (red), GD: Gilbert and Dziewonski (1975), HT: He and Tromp (1996), QM1: observations used in Widmer-Schnidrig et al. (1991), REM: Reference Earth Model website, DE: observations used in Durek and Ekström (1995), RRC: Roult et al. (2010), which only measured $\delta f_{c}$ in (a), and OS: Okal and Stein (2009), which only measured $\delta Q$ in (b).

to compressional velocity $\left(v_{p}\right)$, while about half of the corresponding $l=2$ modes (Fig. 4.3b), are more strongly sensitive to shear velocity $\left(v_{s}\right)$ in the mantle and the inner core. The $l=2$ modes with the highest overtone numbers $\left({ }_{18} S_{2}\right.$ up-to $\left.{ }_{27} S_{2}\right)$ do have dominant sensitivity to compressional velocity $v_{p}$ and have sensitivity kernels which are much more similar to the radial modes. The majority of the $l=2$ spheroidal modes are sensitive to the inner core, and they couple with radial modes for ellipticity and inner core cylindrical anisotropy, which are both degree $s=2$ structure. 


\section{Sensitivity Kernels}
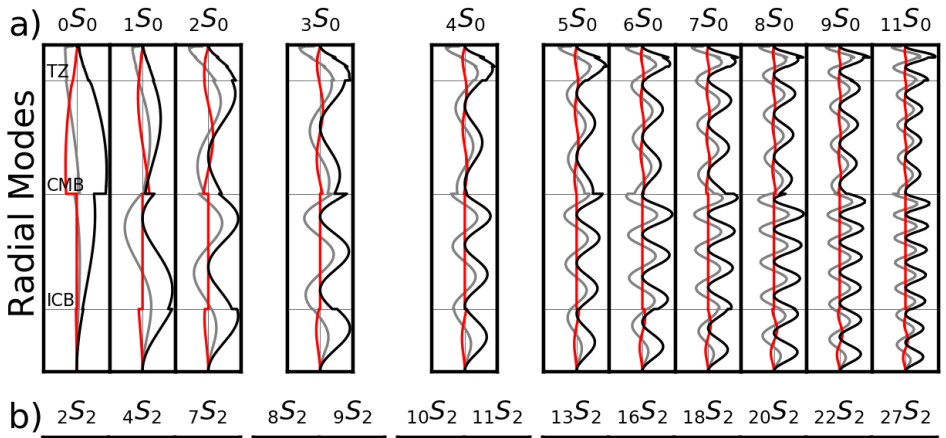

${ }_{10} S_{2}{ }_{11} S_{2}$

${ }_{13} S_{2}{ }_{16} S_{2}{ }_{18} S_{2}{ }_{20} S_{2} \quad{ }_{22} S_{2} \quad{ }_{27} S_{2}$
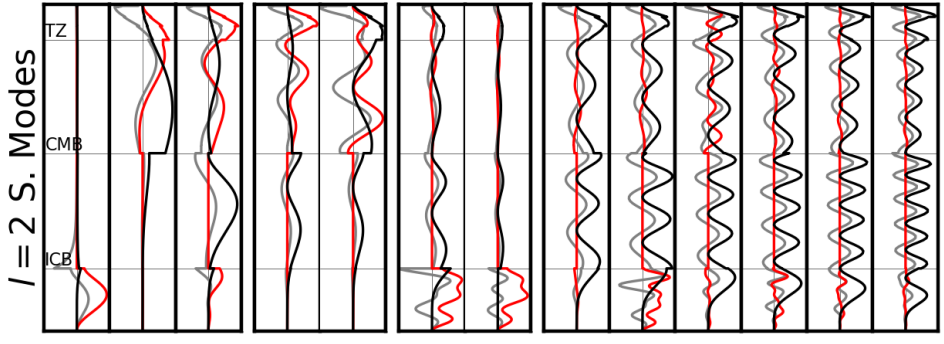

Figure 4.3: Depth sensitivity kernels of density in grey $(\rho)$, shear velocity in red $\left(v_{s}\right)$ and compressional velocity in black $\left(v_{p}\right)$, calculated from PREM. For (a) radial modes and (b) $l=2$ modes coupling to radial modes. Transition Zone (TZ), Core Mantle Boundary $(\mathrm{CMB})$ and Inner Core Boundary (ICB) marked in figure.

In order to validate our GC results, we conducted tests in which we measure radial and $l=2$ mode pairs both in SC and GC using synthetic data (see Figs. 4.4-4.5). The synthetic data are calculated including cross-coupling for ellipticity between the radial modes and their corresponding $l=2$ modes (Table 4.2) in addition to center frequency shifts for the radial modes, they do not include noise effects. In these tests, we are able to recover the synthetic cross-coupling structure between the mode pairs. Similarly, when cross-coupling is not present in the synthetic data, but is included in the splitting function measurement, we do not recover cross-coupling structure. ${ }_{0} S_{0}$ is the only mode where it was not possible to recover synthetic cross-coupling structure, which we also observe using our real data. Following the same strategy used for all other mode pairs and described in Section 4.2, we attempted to measure mode ${ }_{0} S_{0}$ in GC with modes ${ }_{2} S_{2},{ }_{1} S_{2}$ and ${ }_{0} S_{5}$, which Rosat et al. (2007) showed cross-couple strongly with ${ }_{0} S_{0}$. However, in all cases we were unable to measure their cross-coupling using our technique. This is especially evident in tests where cross-coupling was included in the synthetics, but still we were not able to retrieve it in the splitting function measurement (Fig. 4.5). In addition to the large frequency distance between these mode pairs $(>0.124 \mathrm{mHz})$, another reason for this is most likely that modes ${ }_{2} S_{2},{ }_{1} S_{2}$ and ${ }_{0} S_{5}$ have a much smaller $Q$ than ${ }_{0} S_{0}$, and therefore decay much faster and are not visible any more after one or two days, while 83 


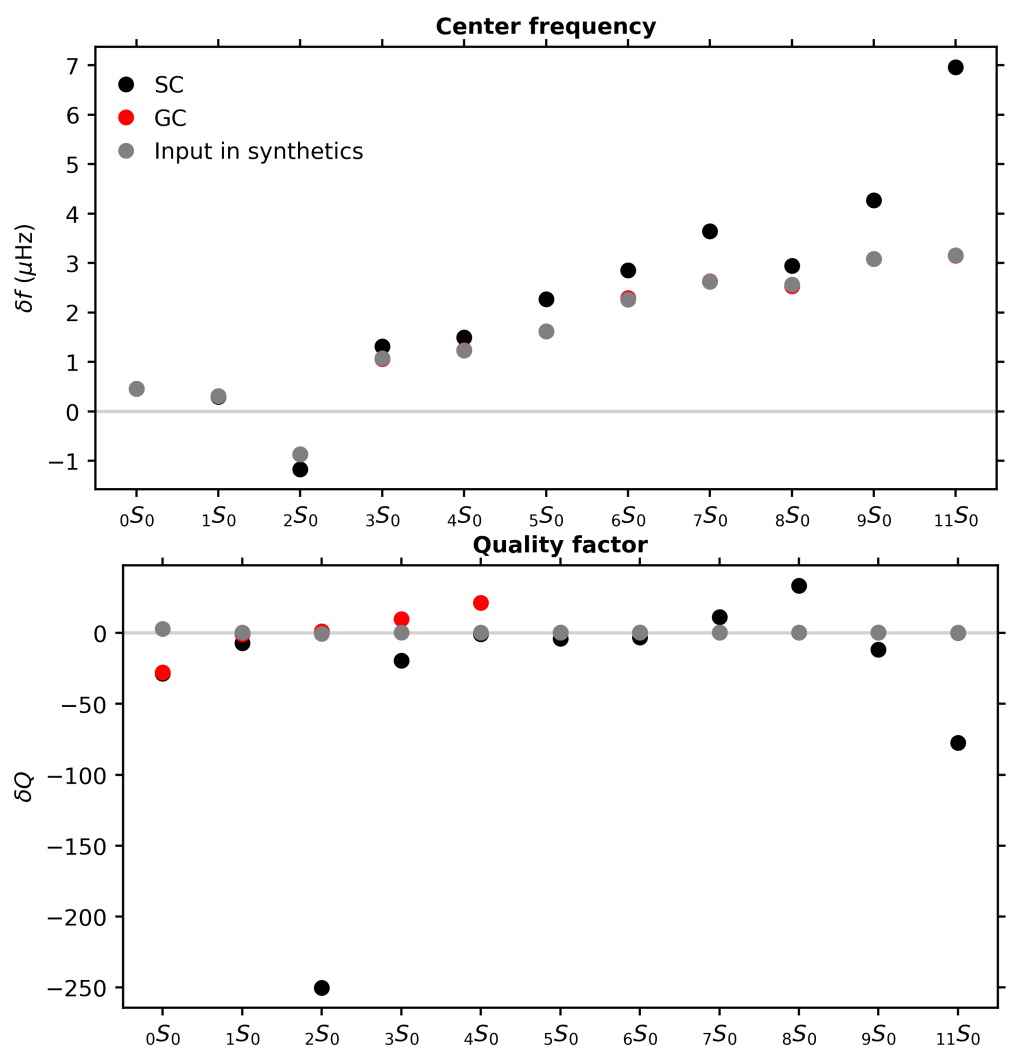

Figure 4.4: Recovery tests where we use synthetic data to measure $f_{c}$ and $Q$ in selfcoupling (SC, black) and group-coupling (GC, red) plotted with respect to PREM. The synthetic data are calculated including cross-coupling due to ellipticity between the radial modes and their corresponding $l=2$ modes (Table 4.2) in addition to center frequency shifts for the radial modes. The centre frequencies measured from the synthetic data using GC (red) match the input center frequency shifts in the synthetic data (grey), while all measurements made using only SC find center frequency shifts from PREM which are too large, just like in our real data measurements. The $Q$ values measured using the synthetic data do not show a systematic difference between SC and GC recovered values. The biggest difference between SC and GC recovered $Q$ values is visible for ${ }_{2} S_{0^{-} 7} S_{2}$ and ${ }_{11} S_{0-27} S_{2}$, which are both strongly cross-coupled modes pairs and are close in frequency. We are not able to fully recover the $Q$ values of ${ }_{0} S_{0},{ }_{3} S_{0}$ and ${ }_{4} S_{0}$, even when we are using GC, which we believe is because of insufficient data. We include the GC $Q$ value deviations from the synthetic test in our uncertainties for these modes.

days are required to properly measure ${ }_{0} S_{0}$. For modes with $n>0$ (Fig. 4.4), we observe a strong trade-off between the cross-coupling $c_{20}$ coefficient (i.e. degree-two structure) and the radial modes' splitting function coefficient $\operatorname{Re}\left(c_{00}\right)$ (i.e. the center frequency), but not with $\operatorname{Im}\left(c_{00}\right)$ (i.e. the quality factor $Q$ ). We see the same effect in our measurements using real data, which we will explain in detail in this section. 


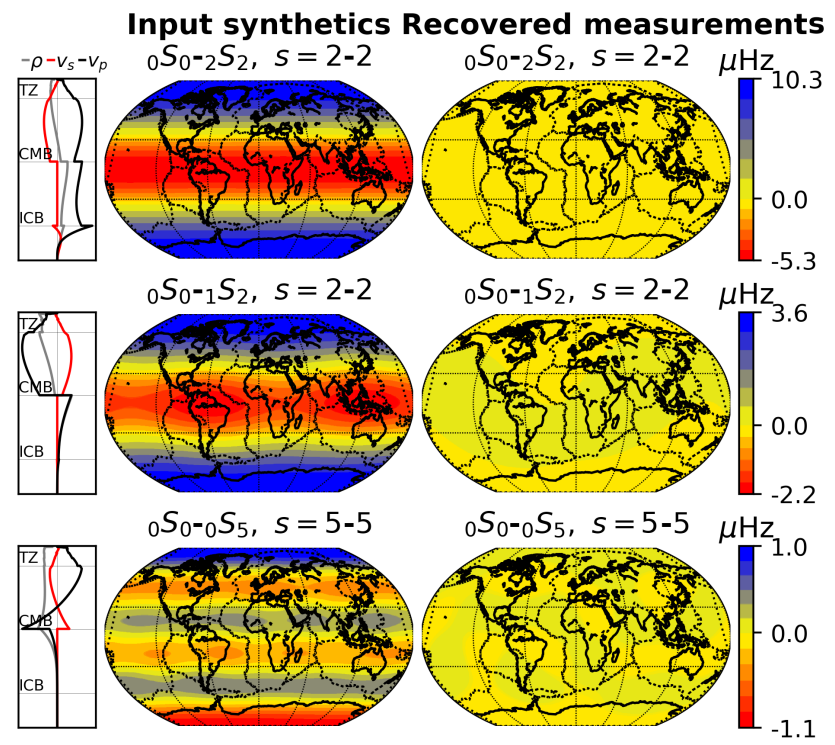

Figure 4.5: Recovery tests using synthetic data which include cross-coupled splitting functions. The synthetic data are calculated including cross-coupling due to ellipticity between the radial modes and their corresponding $l=2$ modes (Table 4.2) in addition to center frequency shifts for the radial modes. The left column shows the cross-coupled splitting functions used to calculate the synthetic spectra. The right column shows the cross-coupled splitting functions measured from these synthetic spectra. Even though crosscoupled structure was included in the synthetic spectra calculation, it was not recovered in the measured splitting functions. In the test, we used the same set-up as what we use to measure cross-coupling in real data, including different frequency windows for both modes. Both in the real data and in the synthetic test shown here, we were not able to recover any cross-coupling between radial mode ${ }_{0} S_{0}$ and ${ }_{2} S_{2},{ }_{1} S_{2}$ or ${ }_{0} S_{5}$.

All the modes measured in SC (Section 4.4.1), are here measured again in GC, with the addition of ${ }_{11} S_{0-27} S_{2}$ (Figs. 4.1c, 4.1f). Radial mode ${ }_{3} S_{0}$ cross-couples to ${ }_{8} S_{2-9} S_{2}$, and ${ }_{4} S_{0}$ cross-couples to ${ }_{10} S_{2-11} S_{2}$. These $l=2$ modes are pairs of inner core sensitive modes, which have almost identical frequency and are very strongly coupled for structure up-to degree 4 . They also cross-couple to their respective radial mode for degree-two structure. Andrews et al. (2006), performed synthetic tests on these mode pairs which are of the type ${ }_{n \pm 1} S_{2^{-}}{ }_{n} S_{2}$, and found that they are strongly cross-coupled by attenuation. In the case of ${ }_{10} S_{2-11} S_{2}$, which strongly depends on the underlying 1D model used, the attenuation cross-coupling causes ${ }_{10} S_{2}$ to increase its $Q$ value significantly, which allows it to be visible in the spectra. The ${ }_{n \pm 1} S_{2^{-}{ }_{n}} S_{2}$ modes used here were first measured in pairs (see Chapter 3), and those results have been used as a starting model here in our GC measurements.

All radial modes and their $l=2$ spheroidal modes have been cross-coupled for rotation and ellipticity as predicted by PREM. In the case of modes ${ }_{0} S_{0},{ }_{6} S_{0},{ }_{7} S_{0}$, ${ }_{8} S_{0},{ }_{9} S_{0}$, we are unable to robustly constrain additional cross-coupling for $s=2$ 
structure to their corresponding $l=2$ modes (see Table 4.3 for misfit $m^{\mathrm{GC}^{e}}$ ). These radial modes couple to ${ }_{2} S_{2},{ }_{16} S_{2},{ }_{18} S_{2},{ }_{20} S_{2},{ }_{22} S_{2}$ respectively, none of which can be measured, either because they are not visible in the data (inner core confined modes) or their visibility is so poor that not enough normal-mode spectra were available to obtain a stable measurement. Radial modes ${ }_{1} S_{0},{ }_{2} S_{0},{ }_{3} S_{0},{ }_{4} S_{0},{ }_{5} S_{0}$ and ${ }_{11} S_{0}$ were all cross-coupled for $s=2$ structure because enough spectra were available for their corresponding $l=2$ modes.

Fig. 4.2a shows our $f_{c}$ and $Q$ measurements along the radial mode branch as a function of overtone number $n$. We observe smoother variations in centre frequency $f_{c}$ in our GC than in our SC measurements; modes ${ }_{2} S_{0}$ and ${ }_{5} S_{0}$ are outliers in the SC measurements and move towards a smoother variation along the branch in the GC measurements. Thus, including GC results in a much-improved branch consistency between all radial mode $f_{c}$ measurements. The cross-coupling effect on the modes becomes larger as we move to higher frequencies along the radial branch, which is evidenced when comparing the SC and GC misfits (Table 4.3). For example, the measured cross-coupling of ${ }_{1} S_{0^{-}} S_{2}$ is not as strong as the one exhibited by other mode pairs at higher frequencies. This is reflected both in the amplitude of the $f_{c}$ shift and, as we will see later, also in the size of the cross-coupled splitting function anomalies.

In the past, Laske et al. (2001) explored the effect of cross-coupling on radial modes, and reported that it produces an overall $f_{c}$ shift towards values closer to PREM. We observe a similar behaviour, but not as pronounced as the one outlined in the Laske et al. (2001) abstract. Unfortunately, we are not able to directly compare Laske et al. (2001) abstract results to our measurements. But we do distinguish a systematic shift of the $f_{c}$ towards PREM, as we allow the radial modes to couple with their closest $l=2$ modes. This is noticeable even when just ellipticity is included in $\mathrm{GC}$, but is more evident when we allow cross-coupling for all degree-two structure, as the $f_{c}$ shift is affected by both, as is the case of ${ }_{2} S_{0},{ }_{5} S_{0}$ and ${ }_{7} S_{0}$ (Fig. 4.2a).

As seen in the synthetic tests, the quality factor $Q$, on the other hand, shows no clear pattern for all modes, and sometimes is not affected at all by the cross-coupling (Fig. 4.2b). The effect of cross-coupling on $Q$, is only evident when the GC modes are very close in frequency, as is the case for modes ${ }_{2} S_{0^{-7}} S_{2}$ which cannot be disentangled. We expect that the strong GC between ${ }_{2} S_{0^{-}} S_{2}$, is the reason of the contradicting results for ${ }_{2} S_{0}$ in previous studies (Fig. 4.2b).

Measuring GC in our inversions reduces the misfit to the data, specially as radial modes and their corresponding $l=2$ modes get closer in frequency making their crosscoupling stronger (Fig. 4.1 and Table 4.3). In the cases where all cross-coupling $s=2$ structure could be measured, we confirmed that the misfit reduction is statistically significant using F-statistics (Section 2.3.2 and Table 4.3). However, in some of the cases where cross-coupling could not be robustly measured we see that the misfit remains the same as in the $\mathrm{SC}$ case, e.g. ${ }_{0} S_{0},{ }_{6} S_{0}$. This is to be expected as we attempt to measure cross-coupling between $l=2$ and radial modes only using data for the radial modes.

\subsubsection{2 $l=2$ Modes}

The cross-coupling also allows us to measure splitting functions for the $l=2$ modes and their cross-coupled splitting functions with the radial modes. We measured 
Our measurement Inner core model S20RTS+CRUST5.1

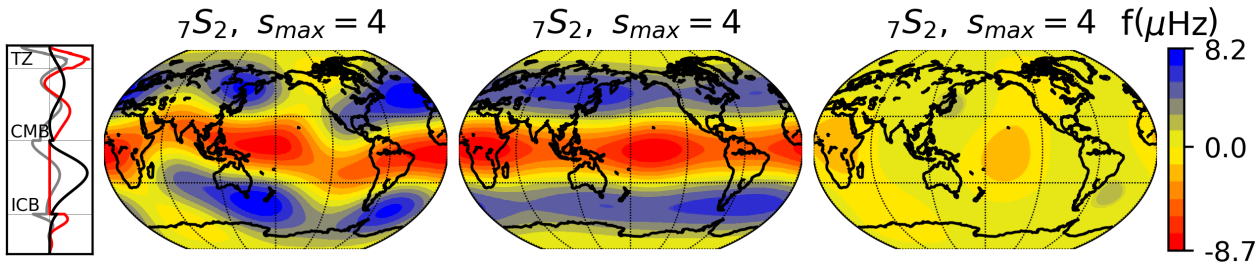

Figure 4.6: Splitting function measurement of mode ${ }_{7} S_{2}$, measured in GC with ${ }_{2} S_{0}$, compared to a combined mantle and inner core prediction (middle) and a mantle prediction (right). Inner core model: Woodhouse et al. (1986).

the splitting function coefficients of modes ${ }_{7} S_{2},{ }_{8} S_{2},{ }_{9} S_{2},{ }_{10} S_{2},{ }_{11} S_{2},{ }_{13} S_{2}$ and ${ }_{27} S_{2}$. Fig. 4.6 shows our splitting function map for mode ${ }_{7} S_{2}$ which has not been reported before. It shows the typical zonal splitting of an inner core sensitive mode, with strong anomalies along the poles and the equator. Masters et al. (2000b) observed the same anomalous splitting for mode ${ }_{7} S_{2}$, as well as its strong cross-coupling to ${ }_{2} S_{0}$, using the singlet frequencies of the mode pair. The splitting functions of the other $l=2$ modes show a similar pattern, see Fig. 4.7 where we compare our $l=2$ observations to Deuss et al. (2013), who measured the modes in SC using the same method, and Pachhai et al. (2020), who also measured the modes in SC but using the matrix autoregressive analysis method. The anomalous zonal splitting is due to strong zonal degree-two $\left(c_{20}\right)$ and degree-four $\left(c_{40}\right)$ values (Figs. 4.8a-4.8b). This typical zonal splitting cannot be satisfied by mantle and crustal predictions, and requires inner core cylindrical anisotropy with the fast axis aligned with the rotation axis of the Earth (i.e. Woodhouse et al., 1986). Overall, when comparing our GC results for the inner core sensitive modes to previous measurements in SC (Deuss et al., 2013; Giardini et al., 1988), we find that coefficients $c_{20}$ and $c_{40}$ have higher values when we include cross-coupling.

\subsubsection{Cross-coupling}

The interpretation of our cross-coupling splitting function maps is not as straight forward as in the self-coupling case. The reason for this is the more complicated nature of their cross-coupling kernels (Fig. 4.9), which cause the signature of inner core anisotropy in a splitting function to change. There are still strong anomalies near the poles and along the equator (so called 'zonal splitting'), but the anomalies near the poles are now negative in some cases, which depends on the specific mode pair. This is not only evident in the observations (left column of Fig. 4.9), but also in the predictions for an inner core anisotropy model with north-south aligned cylindrical inner core anisotropy (middle column of Fig. 4.9). The model predictions show that for ${ }_{1} S_{0^{-} 4} S_{2},{ }_{2} S_{0^{-} 7} S_{2},{ }_{4} S_{0^{-} 10} S_{2},{ }_{4} S_{0^{-} 11} S_{2}$ the polar anomalies are expected to be positive. For ${ }_{3} S_{0^{-} 8} S_{2},{ }_{3} S_{0^{-} 9} S_{2},{ }_{5} S_{0^{-} 13} S_{2}$ and ${ }_{11} S_{0^{-}-27} S_{2}$ the polar anomalies are negative in the model predictions. Also shown for comparison are the models predictions using only a mantle model. Here, no polar anomalies are visible, but again the sign of the anomalies is sometimes opposite to what we usually see for 

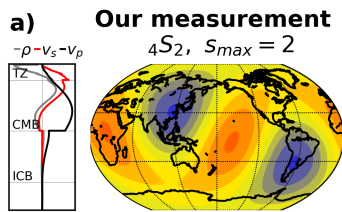

b)
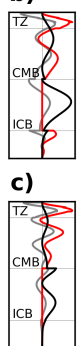

d)

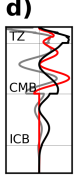

e)
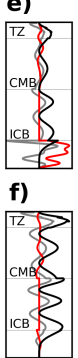

g)

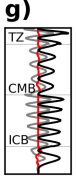

${ }_{7} S_{2}, S_{\max }=4$

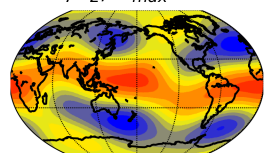

${ }_{8} S_{2}, S_{\max }=4$

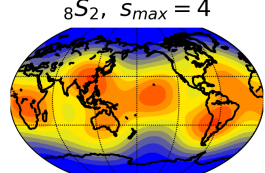

${ }_{9} S_{2}, S_{\max }=4$

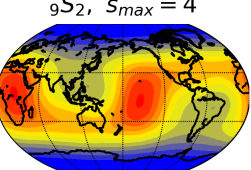

${ }_{10} S_{2}, S_{\max }=4$

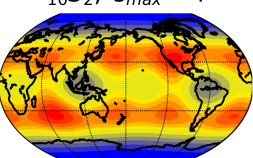

${ }_{13} S_{2}, S_{\max }=4$

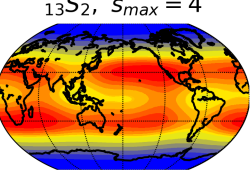

${ }_{27} S_{2}, S_{\max }=4$

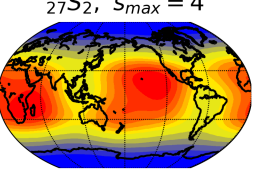

S20+CRUST5.1+IC

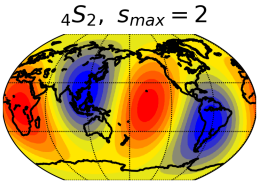

${ }_{7} S_{2}, S_{\max }=4$

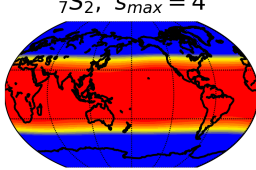

${ }_{8} S_{2}, S_{\max }=4$

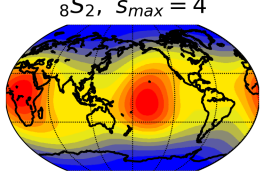

${ }_{9} S_{2}, S_{\max }=4$
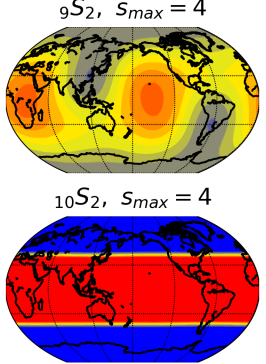

${ }_{13} S_{2}, S_{\max }=4$
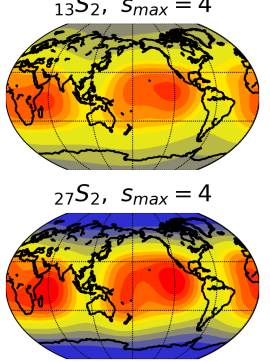

Deuss et al. 2013 Pachhai et al. 2020
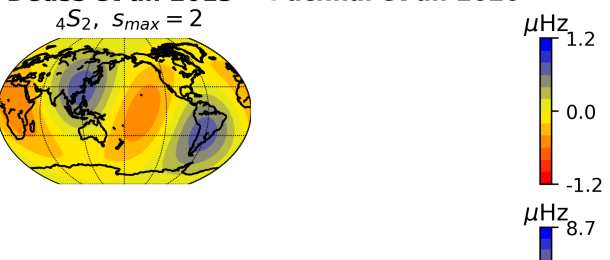

0.0
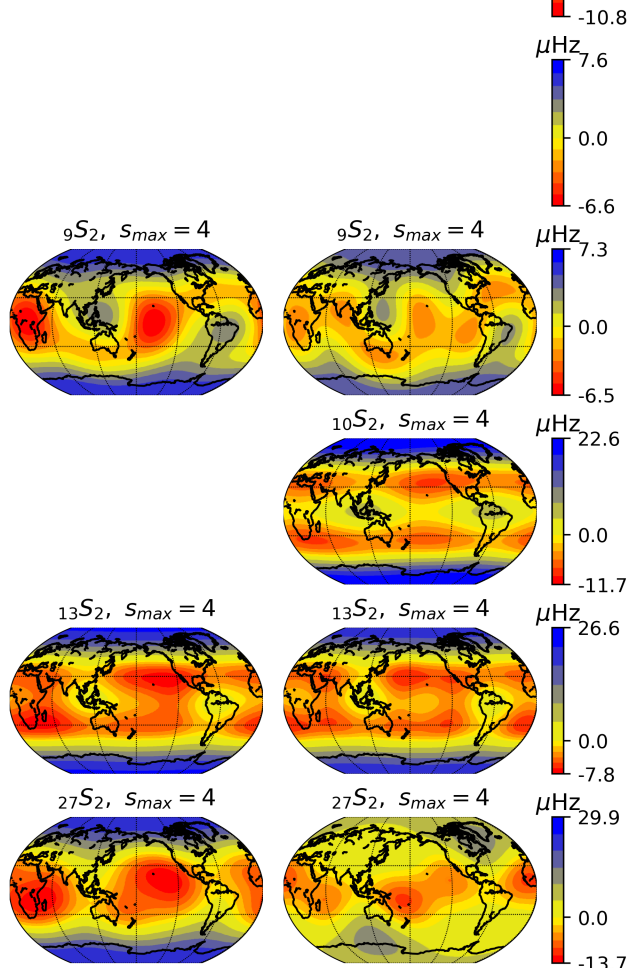

Figure 4.7: Comparison between our self-coupling splitting functions measurements of $l=2$ modes, measured in GC with their respective radial modes, with predictions for a combined mantle and inner core anisotropy model using Tromp (1993) (second column), and previous SC measurement by Deuss et al. (2013) (third column) and Pachhai et al. (2020) (fourth column).

mantle structure (right column of Fig. 4.9). By comparing the model predictions (right and middle columns) with the real observations (left column), we interpret our observations as being due to inner core or mantle structure and if it has the expected sign for such structure. The fact that our observations do not always agree with the model predictions, means that our observations provide new information which may help to improve future models. 
a)

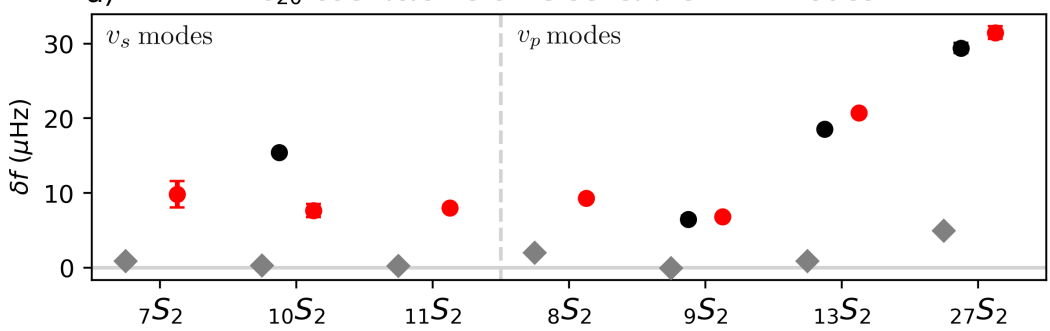

b)

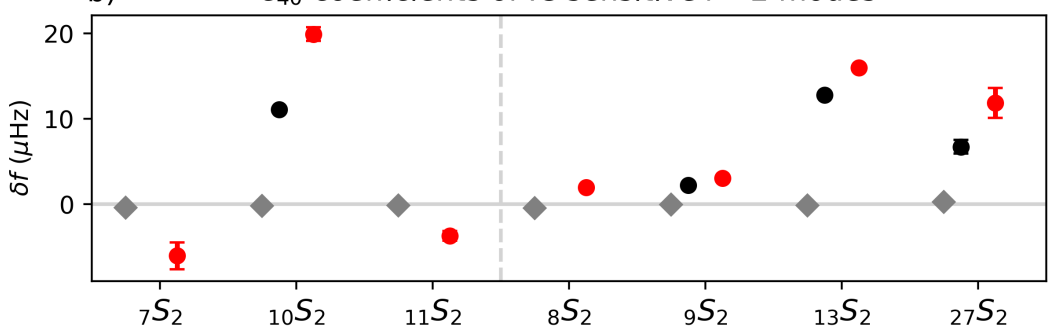

c) $\quad C_{20}$ coefficients of IC sensitive cross-coupling modes

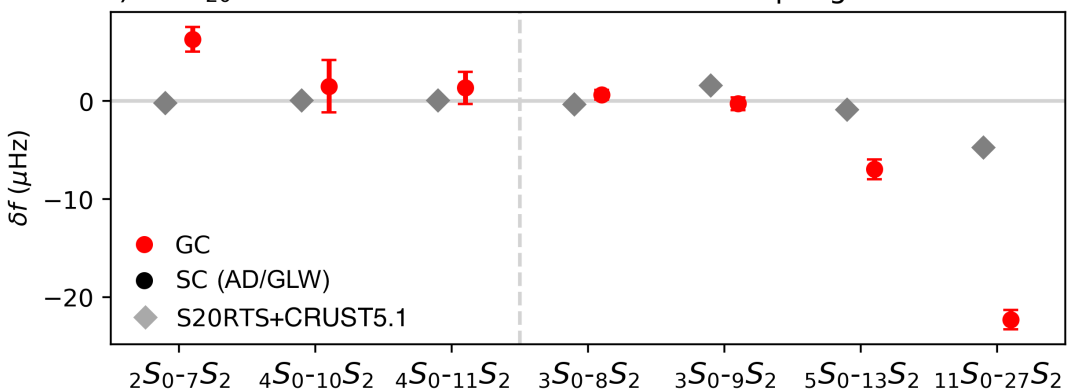

Figure 4.8: $c_{20}$ and $c_{40}$ splitting function coefficients with respect to PREM for (a, b) $l=2$ modes sensitive to the inner core and (c) their corresponding cross-coupling to radial modes. Our GC measurements (red circles) are compare to model predictions and previous measurements.S20RTS: mantle model with crustal corrections (grey diamonds); measurements in self-coupling (black circles) by Deuss et al. (2013) (AD) and Giardini et al. (1988) for ${ }_{10} S_{2}$ (GLW).

In Fig. 4.9, we see that just as for our $l=2$ modes, the cross-coupled splitting function coefficients of ${ }_{2} S_{0^{-} 7} S_{2},{ }_{5} S_{0^{-} 13} S_{2}$ and ${ }_{11} S_{0^{-}}{ }_{27} S_{2}$ also show anomalous splitting due to inner core cylindrical anisotropy, as further seen in the $c_{20}$ cross-coupling coefficients in Fig. 4.8c. Inner core model predictions of $c_{20}$ cross-coupling coefficients follow the same trend as our observations, with mode pairs ${ }_{2} S_{0^{-} 7} S_{2},{ }_{5} S_{0^{-} 13} S_{2}$ and ${ }_{11} S_{0^{-} 27} S_{2}$ predicted to be strongly sensitive to inner core anisotropy. In spite of also being sensitive to the inner core, the cross-coupled splitting functions of ${ }_{1} S_{0^{-}} S_{2}$, ${ }_{3} S_{0^{-} 8} S_{2},{ }_{3} S_{0^{-} 9} S_{2},{ }_{4} S_{0^{-} 10} S_{2},{ }_{4} S_{0^{-} 11} S_{2}$ are dominated by mantle structure, and do not show anomalous zonal splitting near the poles. 
a) Our measurement S20+CRUST5.1+IC S20+CRUST5.1
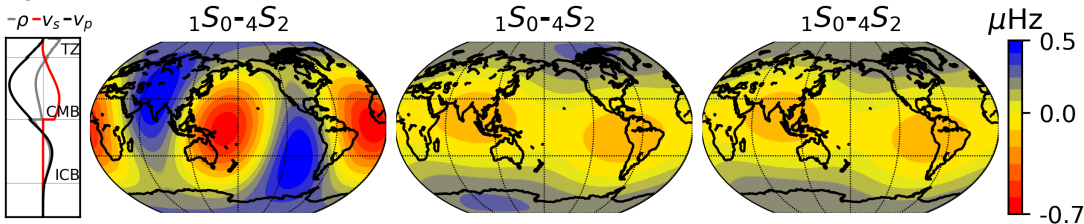

b)
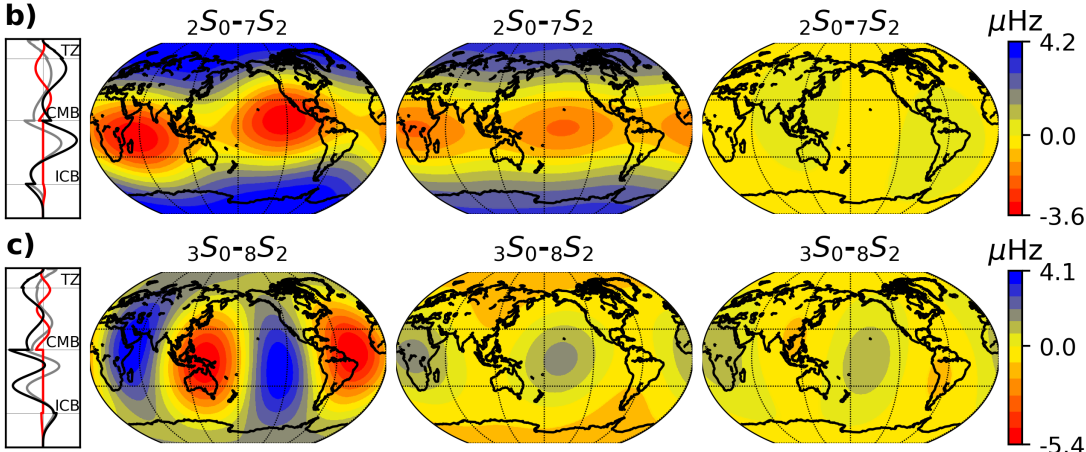

d)

${ }_{3} S_{0-9} S_{2}$

${ }_{3} S_{0-9} S_{2}$
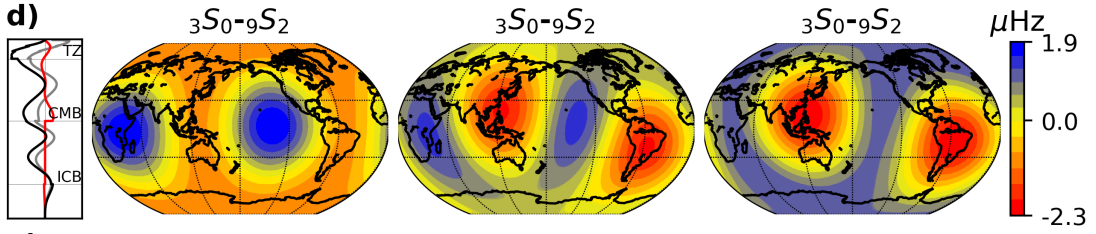

e)

${ }_{4} S_{0}-10 S_{2}$
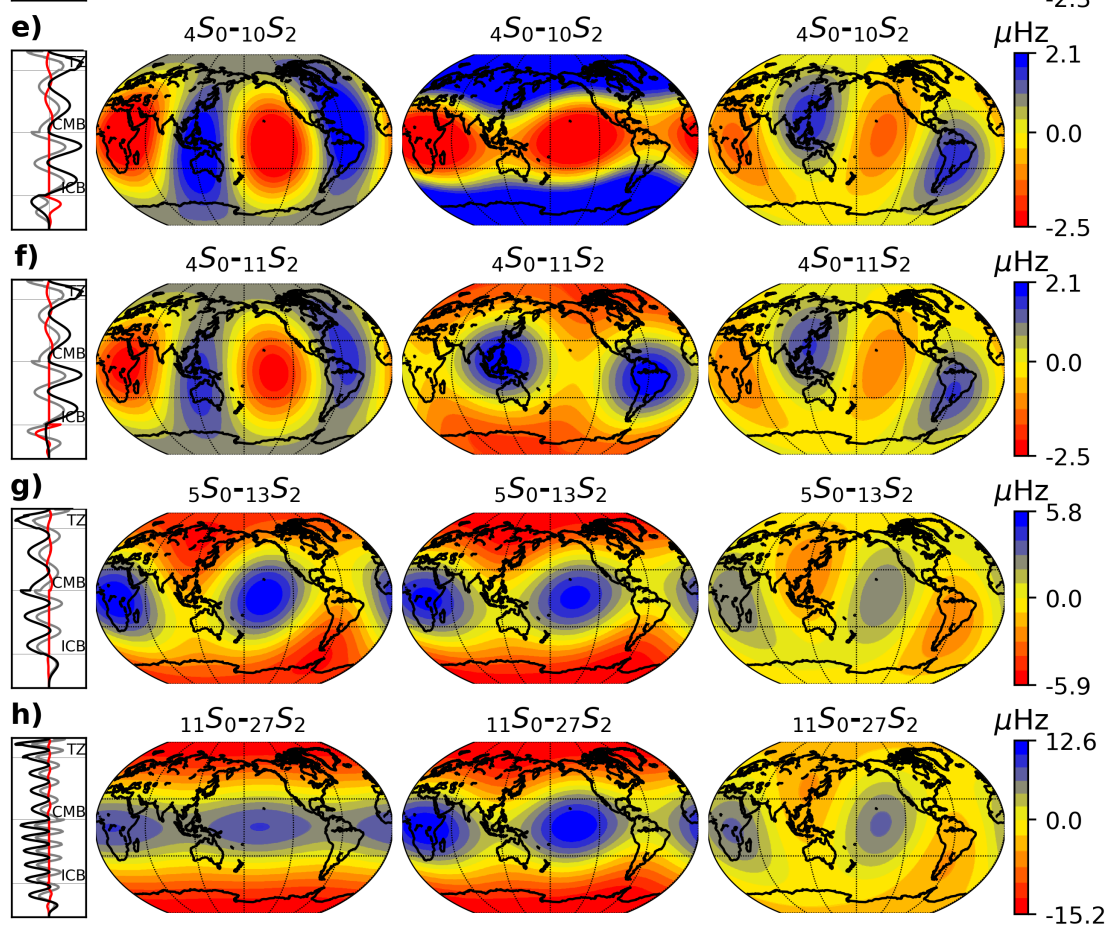

Figure 4.9: Cross-coupling splitting functions measurements between radial modes and $l=2$ modes for $s=2$, compared to a combined mantle and inner core anisotropy model using Tromp (1993) (middle) and only a mantle prediction (right). 


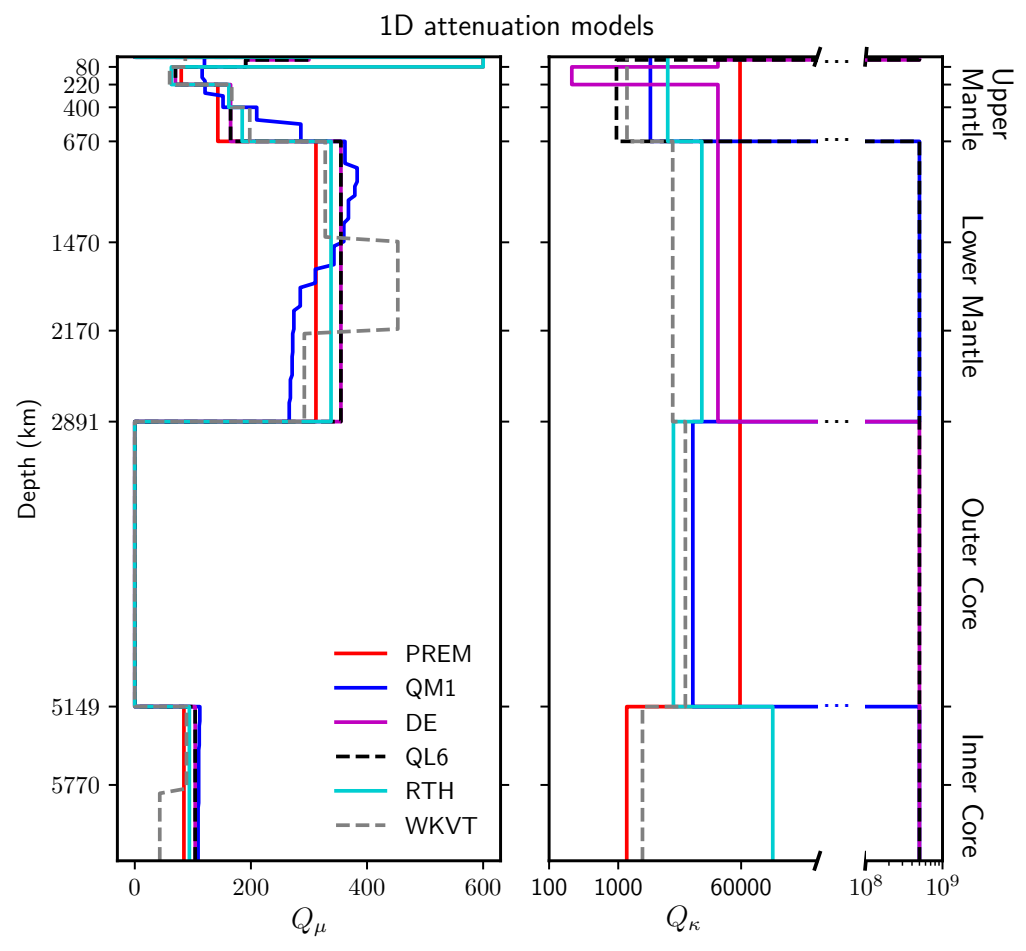

Figure 4.10: 1D attenuation models based on normal modes, for shear attenuation $Q_{\mu}$ (left) and bulk attenuation $Q_{\kappa}$ (right). Models PREM (Dziewonski and Anderson, 1981); QM1 (Widmer-Schnidrig et al., 1991); DE (Durek and Ekström, 1995); QL6 (Durek and Ekström, 1996); RTH (Resovsky et al., 2005); and WKVT (de Wit et al., 2014).

\subsection{Interpretation}

\subsubsection{Bulk attenuation}

The location and strength of bulk attenuation $Q_{\kappa}$ inside the Earth has been highly debated, because the observed $Q$ values of radial modes cannot be explained without the presence of finite $Q_{\kappa}$ somewhere in the Earth. In the past (see Fig. 4.10), finite $Q_{\kappa}$ has been placed in the upper mantle (Sailor and Dziewonski, 1978; Widmer-Schnidrig et al., 1991; Durek and Ekström, 1996), the lithosphere (Durek and Ekström, 1995), the inner core (Anderson and Hart, 1978; Dziewonski and Anderson, 1981), and even the outer core or lower mantle (Resovsky et al., 2005; de Wit et al., 2014).

Now that our radial mode observations have been measured considering the effects of cross-coupling and thus are corrected for these effects, we can directly compare their independent observations to 1D Earth profiles generated without cross-coupling. We calculate $Q$ value predictions for radial modes using a range of $1 \mathrm{D} Q_{\kappa}$ profiles and compare these with our new $Q$ measurements. We do this by recalculating the mode catalogue for the different $1 \mathrm{D} Q_{\kappa}$ profiles using MINEOS 


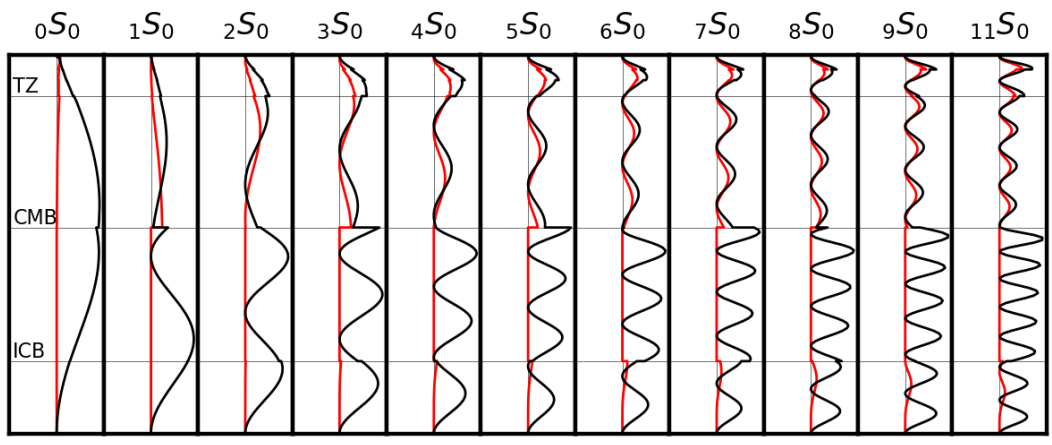

Figure 4.11: Radial mode sensitivity kernels as a function of depth for shear attenuation $q_{\mu}$ in red $\left(\mu_{0} K_{\mu}\right)$ and bulk attenuation $q_{\kappa}$ in black $\left(\kappa_{0} K_{\kappa}\right)$, calculated for PREM. The transition Zone (TZ), Core Mantle Boundary (CMB) and Inner Core Boundary (ICB) are marked in figure.

(Woodhouse, 1988; Masters et al., 2011), while keeping the values of $v_{s}, v_{p}, \rho$ and $Q_{\mu}$ as in PREM. Mode ${ }_{0} S_{0}$ is the most sensitive to variations in $Q_{\kappa}$ (see Fig. 4.11), because it is dominated by compressional energy. Other modes exhibit smaller $Q$ changes as a result of varying $Q_{\kappa}$, specially as we move towards higher frequencies.

We build synthetic $1 \mathrm{D} Q_{\kappa}$ profiles by systematically varying the strength of $Q_{\kappa}$ in the lithosphere, upper mantle, lower mantle, outer core and inner core, one region at a time, while assuming negligible bulk attenuation elsewhere $\left(Q_{\kappa}=5^{8}\right)$ and using PREM's 1D shear attenuation $Q_{\mu}$ profile (Fig. 4.12). We confirm that, as seen in previous probabilistic studies (Resovsky et al., 2005; de Wit et al., 2014), $Q_{\kappa}$ can be placed in different regions of the Earth and yield similar results (Fig. 4.12). Setting $Q_{\kappa}$ to 1200 in the inner core cannot explain the observations of mode ${ }_{0} S_{0}$ and all other radial modes simultaneously (Fig. 4.12a). PREM does have a comparable value in the inner core $\left(Q_{\kappa}=1328\right)$, but it is balanced by setting $Q_{\kappa}=57,822$ elsewhere in the Earth. Placing a $Q_{\kappa}$ value of 18,000 either in the outer core or lower mantle, gives predictions that agree with all radial modes (Figs. 4.12b-4.12c). This is also the case if we set $Q_{\kappa}$ at 1100 in the upper mantle (220-660 km) (Fig. 4.12d). On the other hand, setting $Q_{\kappa}$ to 140 in the lower lithosphere $(80-220 \mathrm{~km})$ only matches the radial mode branch up-to mode ${ }_{3} S_{0}$, while all other radial mode $Q$ values are under-predicted (Fig. 4.12e). Finally, setting $Q_{\kappa}$ to 50 in upper part of the lithosphere $(24-80 \mathrm{~km})$ gives the best fit to the whole radial mode branch (Fig. 4.12f). It is interesting to note that placing finite $Q_{\kappa}$ in the lithosphere, will only produce significant changes if the $Q_{\kappa}$ values are around or below 100 and will mainly affect ${ }_{0} S_{0}$. All of the synthetic $Q_{\kappa}$ profiles have a poor fit with mode ${ }_{2} S_{0}$, possibly because of its strong cross-coupling with mode ${ }_{7} S_{2}$. Even though finite $Q_{\kappa}$ in either the lower mantle or the outer core alone can explain the radial modes observations, both Rayleigh waves and PKP-type modes, which also have significant sensitivity to bulk attenuation, prefer finite $Q_{\kappa}$ to be placed in the upper mantle (Durek and Ekström, 1995). Based on this, we propose that our best possible synthetic scenario is setting $Q_{\kappa}=1300$ in 
the upper mantle and lithosphere (Fig. 4.12g), following the depth parametrisation of the 1D $Q$ model QL6 (Durek and Ekström, 1996). The selected 1D $Q_{\mu}$ profile also has an effect in the $Q$ value predictions of radial modes, however, it is beyond the scope of this chapter to find the best fitting $1 \mathrm{D} Q_{\mu}$ model for the radial modes and other types of data.

We also compare our radial mode observations to previous $1 \mathrm{D} Q_{\kappa}$ and $Q_{\mu}$ models based on normal modes (Widmer-Schnidrig et al., 1991; Durek and Ekström, 1995; Durek and Ekström, 1996; Resovsky et al., 2005). We exclude the most recent model by de Wit et al. (2014), as it did not include any radial mode measurements in its construction. Previous models agree rather well on the $1 \mathrm{D} Q_{\mu}$ profile, but vary significantly for $Q_{\kappa}$, ranging in location and several orders in magnitude for the finite $Q_{\kappa}$ (Fig. 4.10). All models under-predict the $Q$ value of ${ }_{0} S_{0}$ (Fig. 4.12h), even when compared to previous observations used to build model QM1 (Widmer-Schnidrig et al., 1991). In particular, RTH, the most likely model of Resovsky et al. (2005)'s probabilistic study, predicts a $Q$ value for mode ${ }_{0} S_{0}$ of just 3700, much lower than any measurement of ${ }_{0} S_{0}$. Our observation of mode ${ }_{0} S_{0}$ indicates that less bulk attenuation (higher $Q_{\kappa}$ ) is needed to explain the radial mode $Q$ value observations. This matches our synthetic tests, where our proposed best scenario has $Q_{\kappa}=1300$ in the upper mantle and lithosphere, much higher than $Q_{\kappa}=943$ in model QL6.

The fact that mode ${ }_{0} S_{0}$ is completely dominated by bulk attenuation, makes it a particularly interesting and important mode to analyse. We used 25 stations (Table 4.3), containing 2000 hours of uninterrupted data, and measured a $Q$ value of $5982_{-174}^{+211}$, which is higher than other recent measurements (Widmer-Schnidrig et al., 1991; Laske et al., 2005; Okal and Stein, 2009). However, our measurement is, within error bars, as large as older measurements of Riedesel et al. (1980) with $5700 \pm 285$, and Zürn et al. (1980) and Knopoff et al. (1979) with 6687 \pm 869 . Given the mode's importance when modelling $Q_{\kappa}$, we confirmed our $Q$ value observation of ${ }_{0} S_{0}$ by re-measuring it in the time domain using 23 out of our 25 available stations following the method of Okal and Stein (2009), who only included data from the great 2004 Sumatra earthquake. We obtain a value of $Q=6018_{-42}^{+72}$ by measuring the modes' decay rate in the time domain, which agrees with our measured value in the frequency domain. Special attention should be given to mode ${ }_{0} S_{0}$ when modelling $1 \mathrm{D} Q_{\kappa}$ and calculating its uncertainties, as this mode is particularly sensitive to $Q_{\kappa}$ strength and location in the Earth.

\subsubsection{Radial anisotropy in the inner core}

Centre frequencies of radial modes have previously been used to constrain inner core radial anisotropy (Lythgoe and Deuss, 2015). Radial anisotropy results in seismic waves travelling at different velocities along the direction of the Earth's radius and perpendicular to it. This type of anisotropy might be present in the upper most $100 \mathrm{~km}$ of the inner core and caused by the alignment of crystals at the surface of the inner core, due to deformation induced by flow stresses and resulting in a fast velocity axis along the Earth's radius (Yoshida et al., 1996). 
a) Inner Core

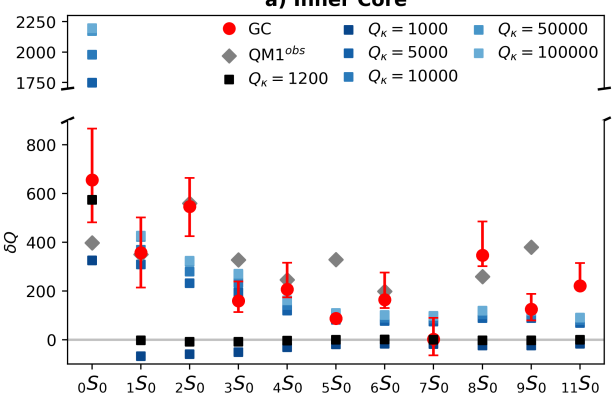

c) Lower Mantle

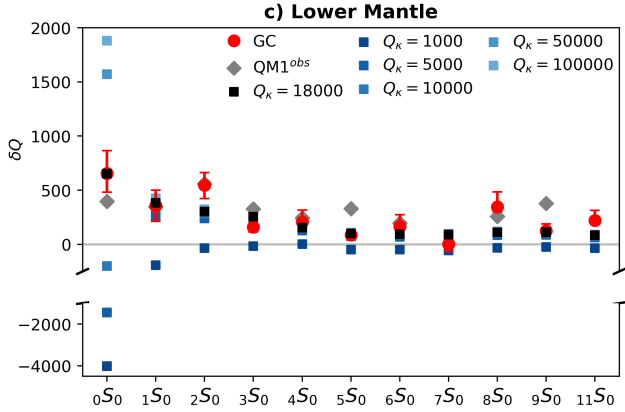

e) Lower Lithosphere (80-220 km)
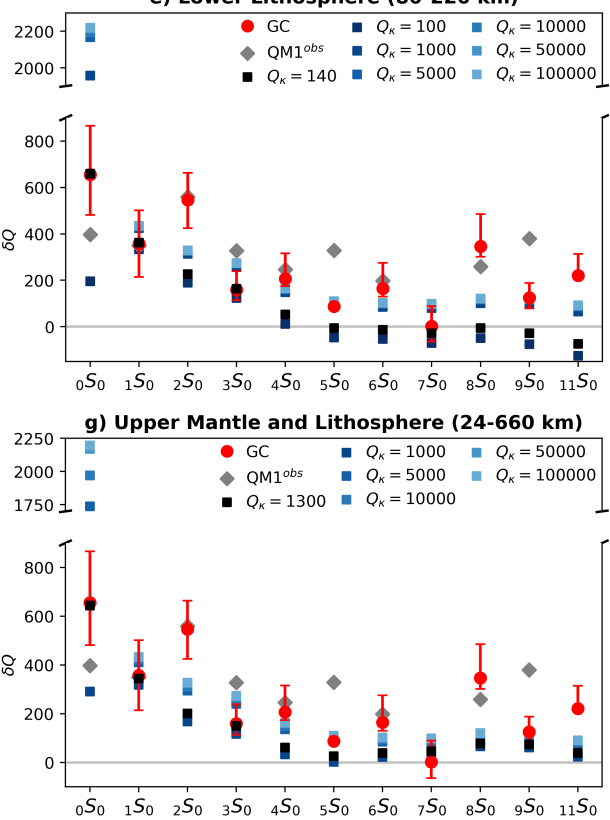

b) Outer Core

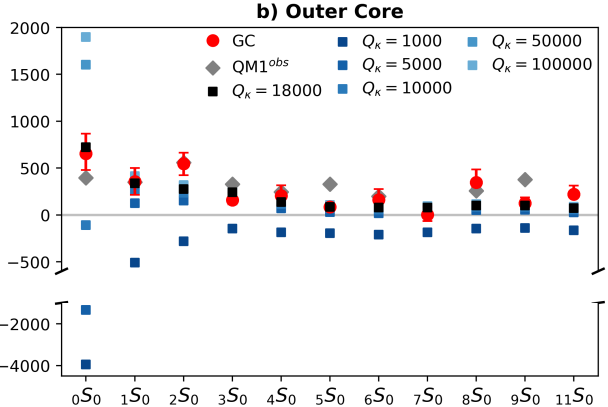

d) Upper Mantle (220-660 km)

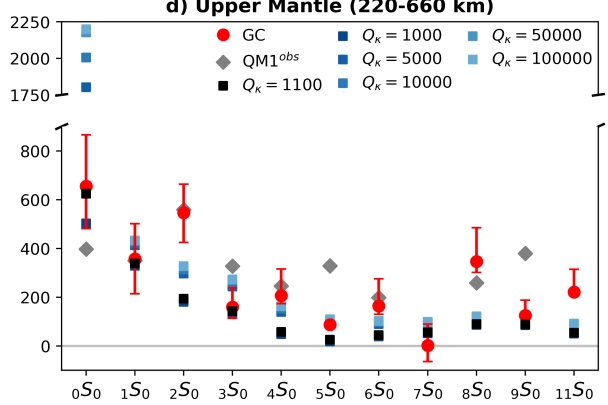

f) Upper Lithosphere (24-80 $\mathbf{~ k m})$
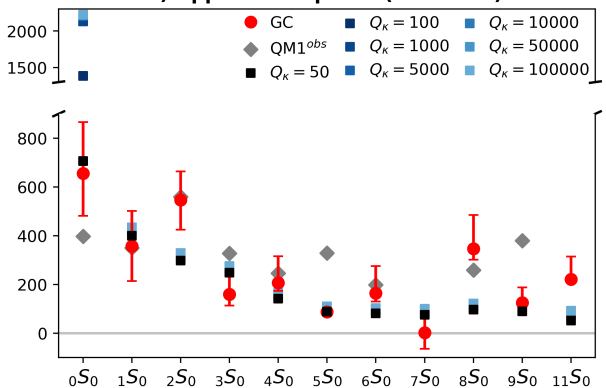

h) 1D Q models

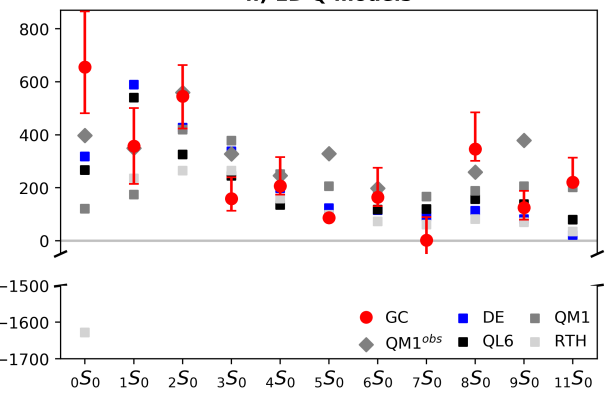

Figure 4.12: Comparison between $1 \mathrm{D} Q$ model predictions and our GC measurements (red circles), all with respect to PREM. In grey diamonds, the radial mode observations used in model QM1 ${ }^{\text {obs }}$ (Widmer-Schnidrig et al., 1991). In (a-g), $Q_{\kappa}$ is varied in each region, while maintaining $Q_{\kappa}$ at $5^{8}$ in all other regions. The blue squares represent the radial modes $Q$ value predictions for each 1D $Q_{\kappa}$ synthetic profile, and the black squares represent the best synthetic predictions in each region. In (h), the 1D $Q$ model predictions are plotted: DE in blue (Durek and Ekström, 1995), QL6 in black (Durek and Ekström, 1996), QM1 in dark grey and RTH in light grey (Resovsky et al., 2005). 


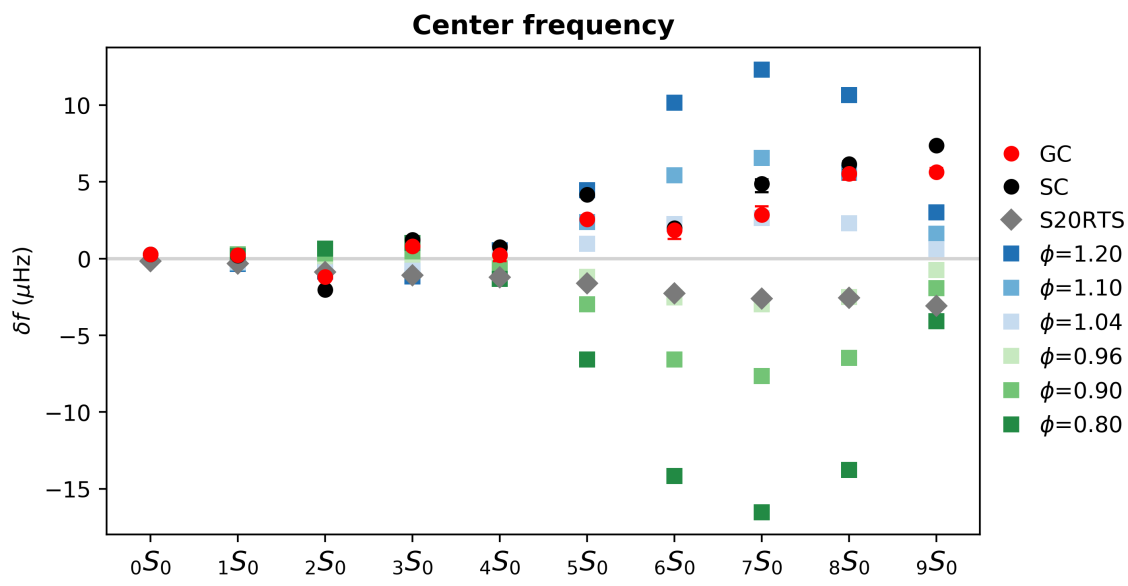

Figure 4.13: Comparison between $f_{c}(\mu \mathrm{Hz})$ predictions obtained by Lythgoe and Deuss (2015) and our SC and GC measurements with respect to PREM. The $f_{c}$ predictions are obtained by varying the radial anisotropy present in the upper most inner core through the parameter $\phi=v_{p v}^{2} / v_{p h}^{2}$, with $v_{p v}<v_{p h}$ in green, $v_{p v}>v_{p h}$ in blue. The PREM line in grey represents no anisotropy and the black markers mantle model S20RTS together with crustal model CRUST5.1.

In Fig. 4.13, we compare our SC and GC results to the $f_{c}$ predictions generated by Lythgoe and Deuss (2015). In their calculations, they vary radial anisotropy in the upper most $100 \mathrm{~km}$ of the inner core by changing the parameter $\phi=v_{p v}^{2} / v_{p h}^{2}$, which changes the proportion between the velocity of compressional waves travelling vertically $\left(v_{p v}\right)$ and horizontally $\left(v_{p h}\right)$ with respect to the inner core boundary. Both our SC and GC results agree with a fast axis along the Earth's radius. However, our results in GC are consistent with lower values of radial anisotropy than previously found by Lythgoe and Deuss (2015). For example, ${ }_{5} S_{0}$ now only requires around $4 \%$ radial anisotropy instead of $9 \%$. Thus, radial mode centre frequencies measured in $\mathrm{SC}$ will result in an overestimation of its radial anisotropy. It is important to note, however, that the isotropic mantle model S20RTS predicts opposite values for the $f_{c}$ shifts than the observations and radial anisotropy predictions. This difference indicates that the radial mode centre frequency observations are more likely due do radial inner core anisotropy than isotropic mantle structure. Moreover, if we take isotropic mantle structure into account even larger values of radial anisotropy would be required to fit our observations.

\subsubsection{Inner core cylindrical anisotropy}

In addition to radial inner core anisotropy, we can also relate our results to cylindrical inner core anisotropy. Previous studies of the Earth's inner core have shown cylindrical velocity anisotropy, with the fast axis roughly aligned to the axis of rotation. In normal modes this is evidenced by a strong zonal degree-two and degree-four structure, which means positive variations in the polar regions, and negative ones in 


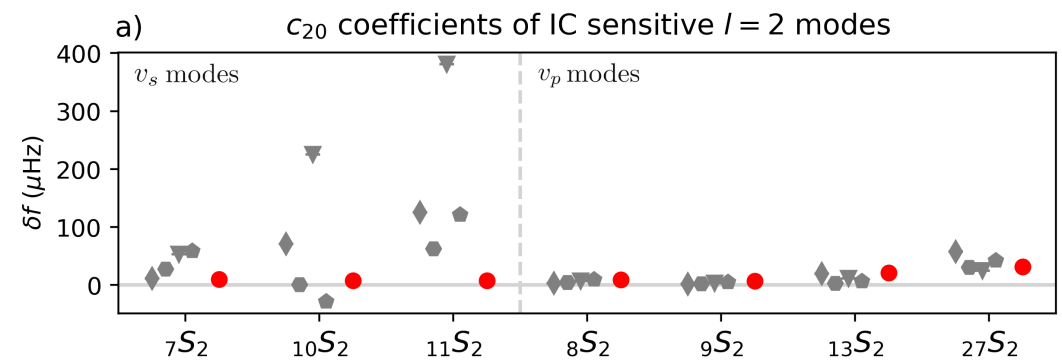

b) $\quad C_{40}$ coefficients of IC sensitive $I=2$ modes

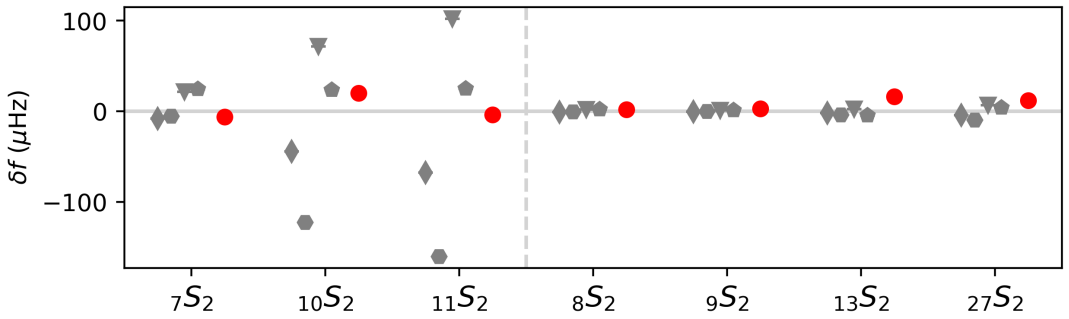

C) $\quad C_{20}$ coefficients of IC sensitive cross-coupling modes

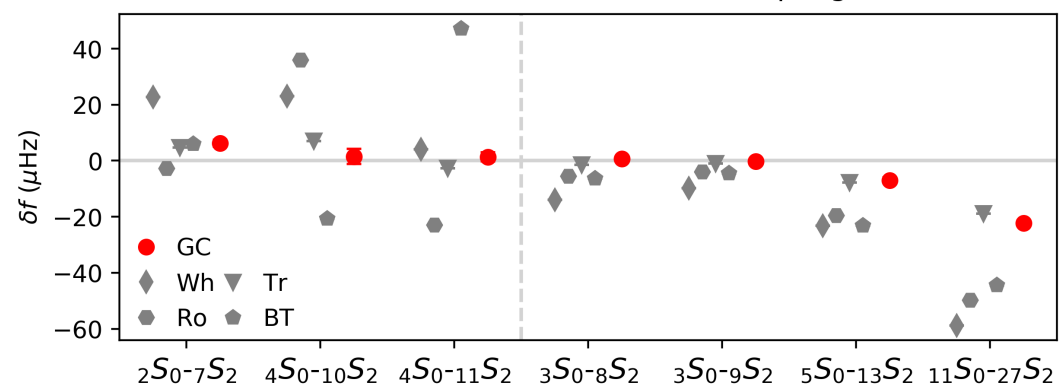

Figure 4.14: $c_{20}$ and $c_{40}$ splitting function coefficients with respect to PREM for $(\mathrm{a}, \mathrm{b})$ $l=2$ modes sensitive to the inner core and (c) their corresponding cross-coupling to radial modes. Our GC measurements (red circles) are compare to inner model predictions in grey: Woodhouse et al. (1986) as Wh; Durek and Romanowicz (1999) as DR; Tromp (1993) as Tr; and Beghein and Trampert (2003) as BT.

the equatorial area. Here, we explore this effect through our observations of crosscoupled splitting functions for radial modes and inner core sensitive $l=2$ modes (pairs ${ }_{n} S_{0^{-}} S_{2}$ ), as they couple both through ellipticity and degree-two structure inner core anisotropy. Some of these cross-coupled mode pairs exhibit the same cylindrical anisotropy behaviour as inner core sensitive modes. Most remarkably, by measuring the cross-coupling between the radial and $l=2$ modes, we observe inner core structure, which is consistent with previous inner core anisotropy models. This is especially the case for pairs where the $l=2$ mode is mostly sensitive to inner core compressional velocity $v_{p}$, such as ${ }_{5} S_{0^{-} 13} S_{2}$ and ${ }_{11} S_{0^{-}-27} S_{2}$ (Fig. 4.8c). On the other hand, $l=2$ modes, which are mostly sensitive to shear velocity $v_{s}$, are often largely over-predicted by current inner core anisotropy models (see modes ${ }_{7} S_{2},{ }_{10} S_{2}$, 
${ }_{11} S_{2}$ and their cross-coupling in Figs. 4.14a-4.14c). This comes as a result of current inner core models being mainly based on $v_{p}$ sensitive modes and/or modes sensitive to the shallow inner core, which makes them unable to provide robust predictions for $v_{s}$ modes sensitive to the innermost inner core. From the available inner core models, we find that all are consistent with all our cross-coupling measurements, with the exception of modes ${ }_{10} S_{2}$ and ${ }_{11} S_{2}$ and their cross-coupling that are not well constrained by any of the models (Fig. 4.14). Thus, important constraints on inner core cylindrical anisotropy can also be obtained from cross-coupling inner core sensitive modes with radial modes, such as mode pairs ${ }_{2} S_{0^{-} 7} S_{2},{ }_{5} S_{0^{-1}} S_{2}$ and ${ }_{11} S_{0-27} S_{2}$ (Figs. $4.8 \mathrm{c}, 4.9$ ).

\subsection{Conclusions}

Unlike previous studies, we measure radial modes not only in self-coupling, but also cross-coupled to their closest $l=2$ spheroidal modes for degree-two structure. We include multiple earthquakes in our measurements, whereas studies before were only able to include either one or two events. By introducing more earthquake data into our measurements, we improve our knowledge of bulk attenuation $Q_{\kappa}$, as we remove potential earthquake bias from our results. Mode ${ }_{0} S_{0}$, which is dominated by $Q_{\kappa}$, has a measured $Q$ value of $5982_{-174}^{+211}$, which is higher than in previous measurements. This indicates that less bulk attenuation, than previously thought, is required in the Earth to explain the observed $Q$ values of radial modes. Other radial modes are not as sensitive to changes in $1 \mathrm{D} Q_{\kappa}$, but are also consistent with less bulk attenuation.

Including cross-coupling in our radial mode measurements results in a systematic shift of the centre frequencies of the radial modes closer to those of PREM. These $f_{c}$ changes, that result from the cross-coupling of modes, have implications on the strength of the radial anisotropy present in the upper most inner core. Our results in group-coupling agree with lower values of radial anisotropy than the ones inferred from just self-coupling. In addition, cross-coupling between radial modes and $l=2$ inner core sensitive modes provide constraints on cylindrical anisotropy, that will help us improve our knowledge of the 3D structure of the inner core. 


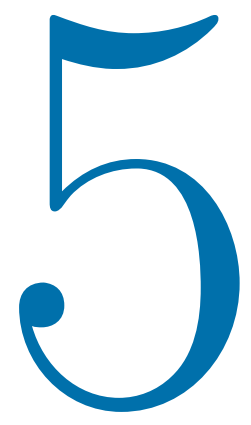

\section{Synthetic tests for constraining $3 \mathrm{D}$ variations in mantle attenuation}

Robust estimates of the Earth's temperature, composition and partial melt are difficult to obtain from seismic tomography models based only on wave velocities. Seismic attenuation can add crucial additional information and allow us to constrain $3 \mathrm{D}$ variations in temperature as well as answer fundamental questions regarding the presence of water and partial melt within the mantle. A major problem with measuring attenuation is that scattering and focusing effects need to be included in order to distinguish between intrinsic attenuation (transformation of energy to heat) and scattering (redistribution of energy). Here, we will use normal modes, exploiting the fact that small scale scattering becomes less important at longer periods. In addition, here, when simultaneously measuring the elastic and anelastic splitting function of a given normal mode, focussing is implicitly included. Normal mode data has rarely been used before to constrain $3 \mathrm{D}$ variations in attenuation, hence we first need to test the feasibility of using whole Earth oscillations to image 3D variations in attenuation in Earth's upper and lower mantle. Simple models are used in forward calculations to evaluate the sensitivity of normal modes to 3D attenuation variations. We also test if $3 \mathrm{D}$ attenuation variations can be recovered using splitting function measurements for synthetic normal mode spectra and investigate potential problems with unaccounted earthquake magnitude variations. 


\subsection{Introduction}

Regional studies of 3D variations in crust and upper mantle attenuation have been performed since the late 1960s (e.g. Molnar and Oliver, 1969; Canas and Mitchell, 1978; Sipkin and Jordan, 1980; Dziewonski and Steim, 1982), where the first evidence was found of a potential anti-correlation between 3D seismic velocity and attenuation variations for different geological provinces. These early studies found young oceanic regions near the spreading ridges with low seismic velocity to be much more strongly attenuating than stable continental regions with high seismic velocity. Later, a global uppermost mantle study using Rayleigh waves found that 3D attenuation in the transition zone is dominated by a well-resolved large degree-two structure (Romanowicz, 1990; Romanowicz, 1987; Roult et al., 1990). This was interpreted as evidence that $3 \mathrm{D}$ shear velocity variations in the upper mantle, which also show the same degree-two pattern, have a thermal origin. However, it would take more than twenty years until the first 3D global tomographic attenuation model was built by Romanowicz (1995). Using Rayleigh waves, they reported large 3D variations in shear attenuation in the upper mantle and linked these to structure found in global 3D shear velocity tomographic models. Just as in the earlier regional studies, Romanowicz (1995) found that regions associated with young oceanic spreading ridges exhibit both lower seismic velocity and stronger attenuation than the surrounding upper mantle, while stable continental shield regions exhibit higher seismic velocity and weaker attenuation.

Since the first study by Romanowicz (1995), eight more global models of upper mantle 3D attenuation have become available (see Fig. 5.1 for three recent models). These global upper mantle studies are most similar in the uppermost $200 \mathrm{~km}$ of the mantle and have been constructed using: fundamental-mode surface waves (Selby and Woodhouse, 2002; Dalton et al., 2008; Adenis et al., 2017); fundamentalmode and overtone surface waves (Gung and Romanowicz, 2004; Karaoğlu and Romanowicz, 2018), with overtones providing greater depth sensitivity; or body waves (Bhattacharyya et al., 1996; Reid et al., 2001; Warren and Shearer, 2002). All these studies require approximation methods to correct for focussing and scattering effects of 3D velocity variations on wave propagation, except when performed using Full-Waveform Inversion (FWI, e.g. Karaoğlu and Romanowicz, 2018). If not properly accounted for, focussing and scattering may map into 3D variations in attenuation, even though this 'apparent attenuation' is actually the result of velocity effects and not due to 'intrinsic attenuation'. In contrast to the upper mantle, there is much less information on lower mantle 3D attenuation, which has only been explored in two published regional body wave studies (Lawrence and Wysession, 2006b; Hwang and Ritsema, 2011), which both did not account for focussing or scattering.

Normal modes have only been employed in a few limited studies before to investigate upper mantle 3D attenuation variations (i.e. Romanowicz et al., 1987; Roult et al., 1990; Masters et al., 2000a; Masters et al., 2000b). Romanowicz et al. (1987) and Roult et al. (1990) used an 'amplitude decay with time' method, and due to data constraints, the attenuation variations of the observed fundamental modes were averaged over three to five consecutive angular orders. The measurements 
5.1 Introduction
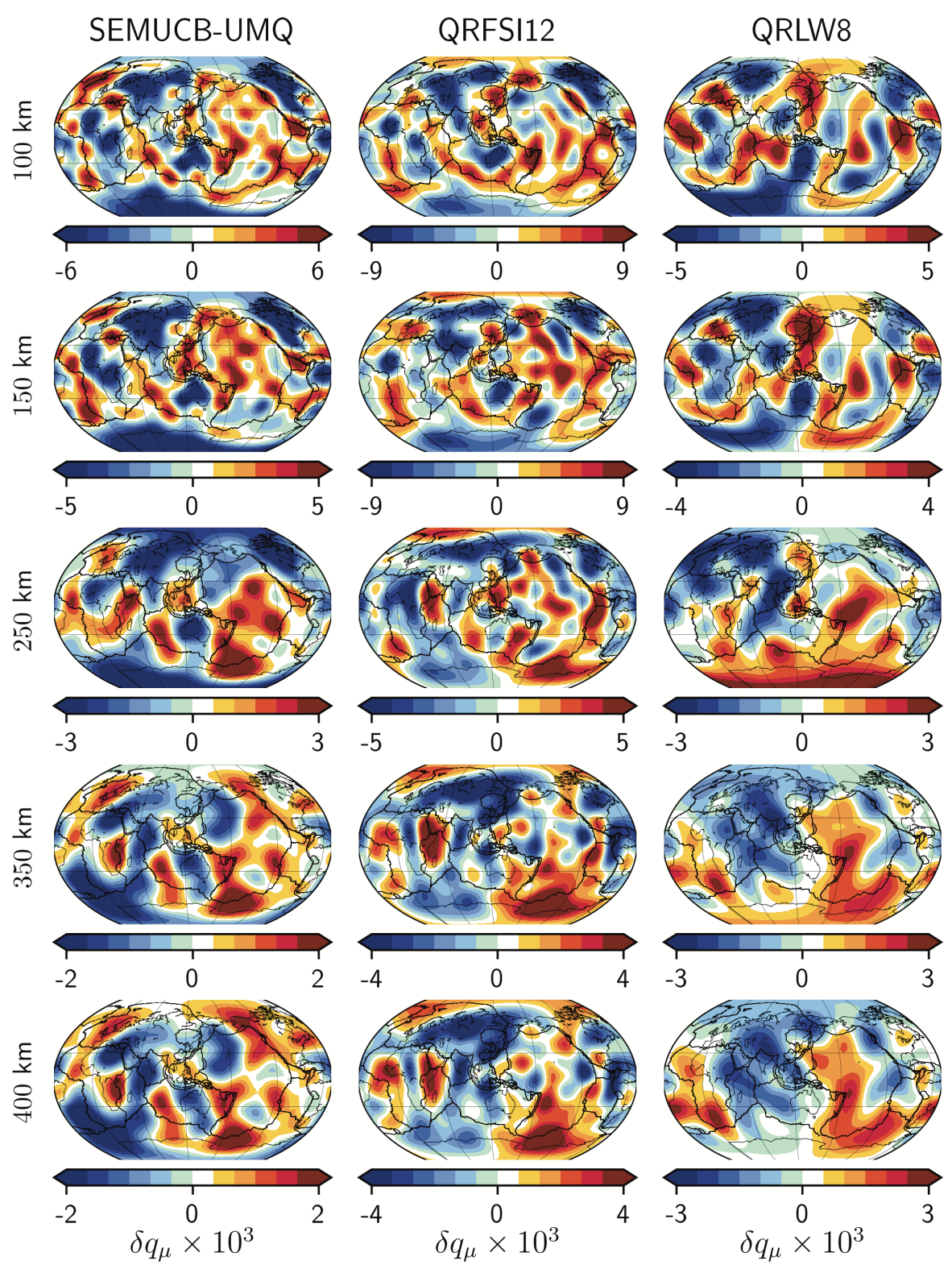

Figure 5.1: Comparison between three 3D global shear attenuation models: SEMUCB-UMQ (Karaoğlu and Romanowicz, 2018), QRFSI12 (Dalton et al., 2008) and QRLW8 (Gung and Romanowicz, 2004). All models are plotted in terms of $\delta q_{\mu} \times 10^{3}$ at five different depths: $100 \mathrm{~km}, 150 \mathrm{~km}, 250 \mathrm{~km}, 350 \mathrm{~km}$ and $400 \mathrm{~km} . \delta q_{\mu} \times 10^{3}$ is presented as perturbation value and in percentage. Tectonic plate boundaries (Bird, 2003) are included for comparison with the $3 \mathrm{D}$ global shear attenuation models. 
of Romanowicz et al. (1987) and Roult et al. (1990) agreed with what was later seen using surface waves studies (e.g. Romanowicz, 1995), and give us confidence that more extensive studies of 3D upper and lower mantle attenuation using normal mode data may be feasible. On the other hand, although Masters et al. (2000a) and Masters et al. (2000b) included 3D anelasticity in their splitting function study using the matrix autoregressive method, they only report and analyse anelastic measurements for the inner core but not the mantle.

Here, we test a different method in which we study $3 \mathrm{D}$ variations in mantle attenuation using the measurement of elastic and anelastic splitting functions (see Chapter 2 for a detailed explanation of our method) and apply it to both upper and lower mantle sensitive modes. First, simple 3D mantle attenuation models are used in forward calculations to test the sensitivity of synthetic normal mode spectra to $3 \mathrm{D}$ variations in attenuation in the whole mantle. Next, we use synthetic spectra, including $3 \mathrm{D}$ variations in attenuation, and test if we can recover these using anelastic splitting function measurements. Finally, we investigate the potential bias by unaccounted earthquake moment magnitude variations on measurements of anelastic splitting functions.

It is important to note, that as seen in Chapter 4 , bulk attenuation $q_{\kappa}$ is relatively small when compared to shear attenuation $q_{\mu}$. 1D $q_{\kappa}$ is difficult to measure even when using normal modes strongly sensitive to compressional energy. Given that $1 \mathrm{D} q_{\kappa}$ is already small when compared to $1 \mathrm{D} q_{\mu}$, we expect its effect to be negligible when investigating 3D attenuation variations in the mantle. For this reason, we will discuss $3 \mathrm{D}$ attenuation only in terms of $q_{\mu}$ in the remaining parts of this thesis.
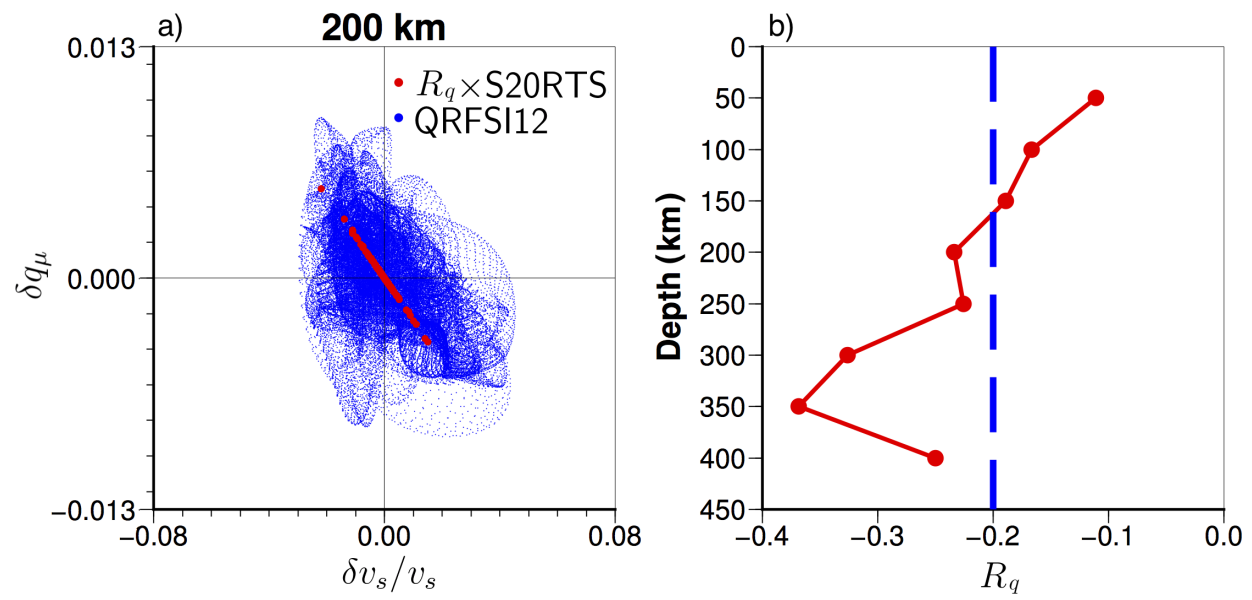

Figure 5.2: (b) Each blue point represents a latitude and longitude location on a grid with a spacing of $1^{\circ}$ in QRFSI12 $(\delta q)$ and S20RTS $\left(\delta v_{s} / v_{s}\right)$ at $200 \mathrm{~km}$ depth. The red points represent the $3 \mathrm{D}$ attenuation synthetic predictions of scaling the $3 \mathrm{D}$ shear velocity model S20RTS by $R_{q}=-0.2$. (b) $R_{q}$ ratio as a function of depth (red line), illustrating the relationship between QRFSI12 (Dalton et al., 2008) and S20RTS (Ritsema et al., 1999). The average value of $R_{q}=-0.2$ is marked as a dashed blue line at all depths. 


\subsection{Forward modelling}

Our aim is to use long-period normal mode data to constrain $3 \mathrm{D}$ variations in attenuation for the whole mantle. So far, only body waves and surface waves have been used to make 3D tomographic models of attenuation in the upper mantle. Thus, we first need to test the feasibility of imaging $3 \mathrm{D}$ variations in attenuation using normal mode data. We will start this by investigating if the effect of $3 \mathrm{D}$ variations in attenuation is large enough to be visible in normal mode spectra using forward modelling of synthetic normal mode spectra.

\subsubsection{Scaling relationship between $q_{\mu}$ and $v_{s}$}

We would like to calculate the signature of current upper mantle 3D attenuation models on synthetic normal mode spectra, and also extrapolate these results to include the lower mantle for which no global 3D attenuation models currently exist. In order to do so, we first explore the relationship between $3 \mathrm{D}$ shear velocity model S20RTS (Ritsema et al., 1999) and upper mantle 3D attenuation model QRSFI12 (Dalton et al., 2008). We define $R_{q}$, which is the ratio relating variations in shear velocity $\delta v_{s}$ and attenuation $\delta q_{\mu}$

$$
\delta q_{\mu}=R_{q} \frac{\delta v_{s}}{v_{s}}
$$

We determine the ratio $R_{q}$ by plotting $\delta v_{s} / v_{s}$ from S20RTS against $\delta q_{\mu}$ from QRFSI12 for points distributed on a regular latitude and longitude grid at different depths in the upper mantle (see Fig. 5.2a for a depth of $200 \mathrm{~km}$ ). We then apply a simple linear fit through the point cloud and use the slope of the fitted line as the $R_{q}$ factor.

We expect to find a negative value for $R_{q}$, in agreement with the anti-correlation between $v_{s}$ and $q_{\mu}$ seen in previous studies for the upper mantle (i.e. Dalton et al., 2009). We indeed find that for the upper mantle, $R_{q}$ is always negative and increases from -0.1 at $50 \mathrm{~km}$ depth, to almost -0.4 at $350 \mathrm{~km}$ depth. We select $R_{q}=-0.2$
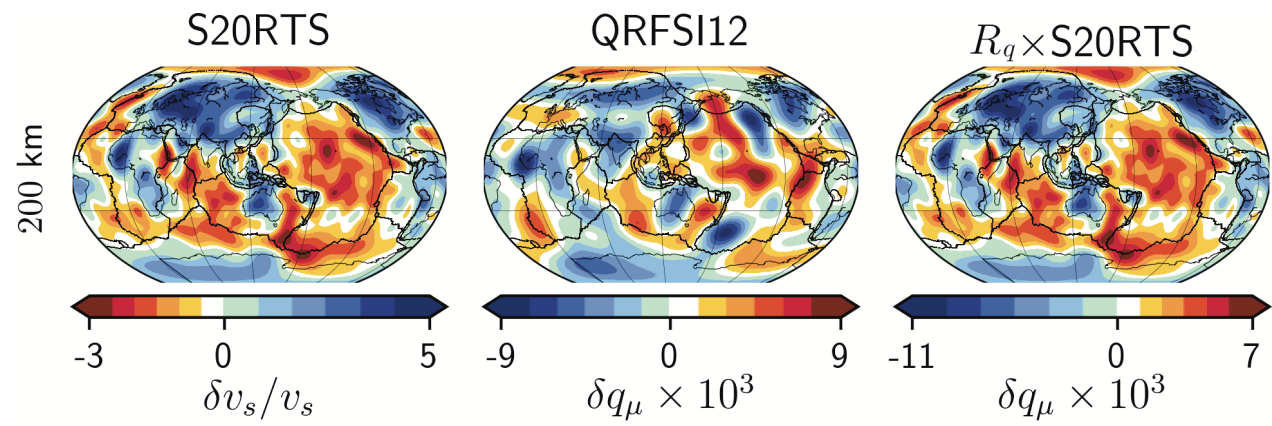

Figure 5.3: From left to right: 3D $v_{s}$ mantle model S20RTS, 3D $q_{\mu}$ upper mantle model QRFSI12, and 3D synthetic $q_{\mu}$ mantle model built by scaling S20RTS using $R_{q}=-0.2$. All models are plotted at $200 \mathrm{~km}$ depth. Tectonic plate boundaries (Bird, 2003) are included for comparison. 

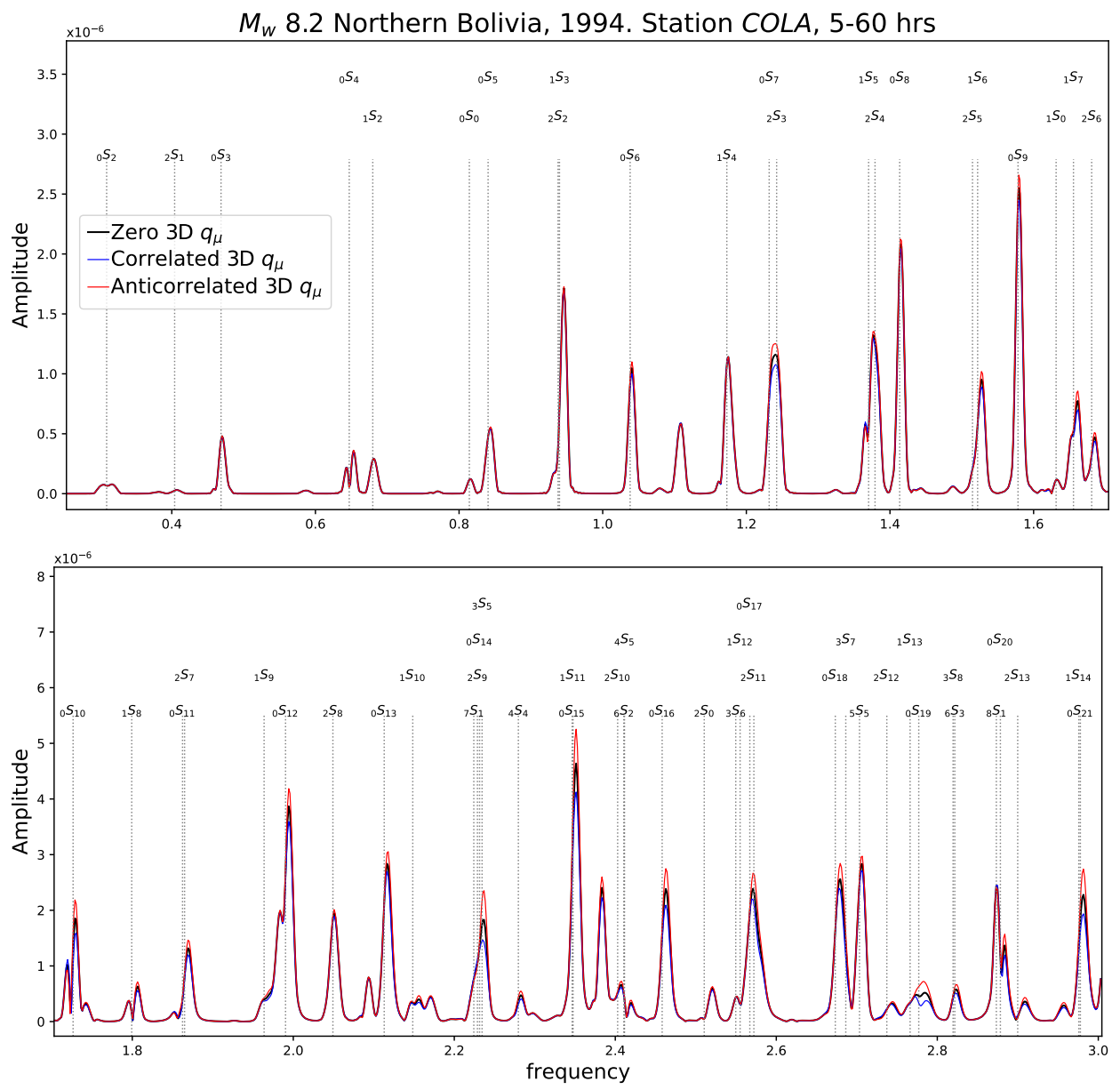

Figure 5.4: Vertical component of the $M_{w} 8.3$ Bolivia earthquake of 1994 for station COLA with a time window of 5-60 h. Full coupling synthetics $(0-3 \mathrm{mHz})$ using elastic model S20RTS (Ritsema et al., 1999) and 3D synthetic anelastic models: anti-correlated $\left(R_{q}=-0.2\right)$ and correlated $\left(R_{q}=0.2\right)$ to S20RTS; and zero 3D $q_{\mu}$ structure $\left(R_{q}=0\right)$.

as the average value (Fig. 5.2b), and extrapolate this value throughout the whole mantle in order to obtain a 3D attenuation model of $\delta q_{\mu}$ by scaling shear wave velocity model S20RTS. In Fig. 5.3, we plot S20RTS and QRFSI12 together with the $3 \mathrm{D}$ attenuation synthetic mantle model built by scaling S20RTS using $R_{q}=-0.2$ for a depth of $200 \mathrm{~km}$. The size of the anomalies in our scaled model is comparable in size to the anomalies in the QRFSI12 model, which justifies our use of the scaled model later in the thesis. 

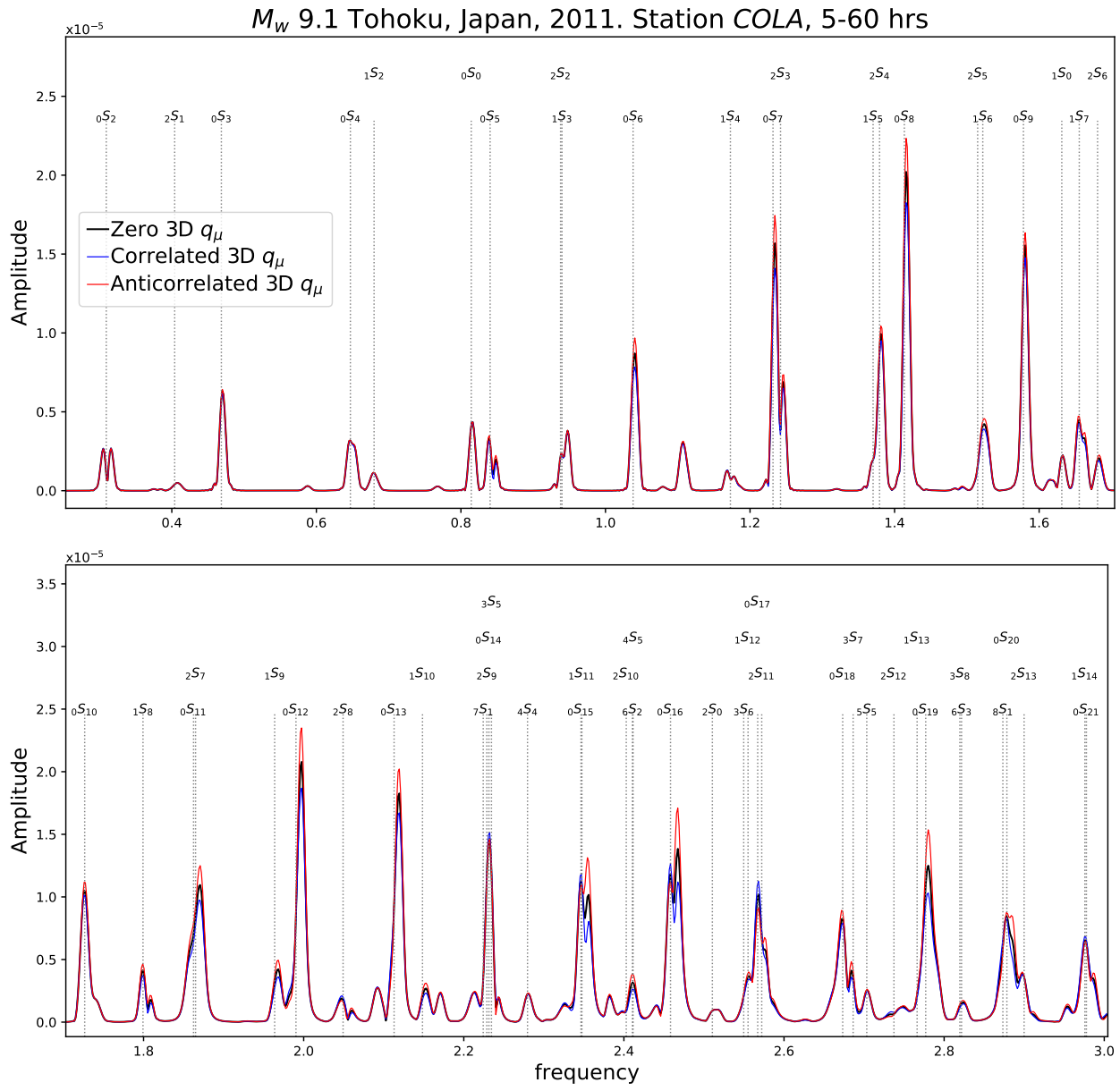

Figure 5.5: Vertical component of the $M_{w} 9.1$ Tohoku earthquake of 2011 for station COLA with a time window of 5-60 h. Full coupling synthetics $(0-3 \mathrm{mHz})$ using elastic model S20RTS (Ritsema et al., 1999) and 3D synthetic anelastic models: anti-correlated $\left(R_{q}=-0.2\right)$ and correlated $\left(R_{q}=0.2\right)$ to S20RTS; and zero 3D $q_{\mu}$ structure $\left(R_{q}=0\right)$.

\subsubsection{Full coupling synthetics}

Next, we analyse the overall sensitivity of normal mode spectra below $3 \mathrm{mHz}$ to attenuation. Our aim is to find out which modes show observable differences when $3 \mathrm{D}$ variations in attenuation are included. This is done by generating full coupling synthetics, including rotation and ellipticity, for the 3D shear wave velocity model S20RTS with (and without) the 3D synthetic anelastic $q_{\mu}$ model obtained by scaling S20RTS with $R_{q}=-0.2,0,0.2$. In Figs. 5.4-5.5, we show full coupling synthetics for the deep $1994 M_{w} 8.3$ Bolivia earthquake and for the shallow $2011 M_{w} 9.1$ Tohoku earthquake. From these full coupling synthetics, we make the following observations: 
(i) modes below $1 \mathrm{mHz}$ show negligible sensitivity to the presence of 3D anelastic structure, with their spectral amplitudes showing only minor changes; (ii) overtones $(n>0)$ above $1 \mathrm{mHz}$ show the largest sensitivity to the presence of $3 \mathrm{D} q_{\mu}$ structure and (iii) fundamental modes $(n=0)$ excited by deep earthquakes (e.g. $M_{w} 8.3$ Bolivia at $647 \mathrm{~km}$ ) do not show large amplitude variations as a result of $3 \mathrm{D} q_{\mu}$ perturbations, ( ${ }_{0} S_{7}$ might appear as an exception, but its amplitude variations are due to mode ${ }_{2} S_{3}$ ), while fundamental modes excited by shallow earthquakes (e.g. $M_{w}$ 9.1 Tohoku at $20 \mathrm{~km}$ ) do show larger amplitude variations to $3 \mathrm{D} q_{\mu}$ perturbations.

Fundamental modes $(n=0)$ with $l<7$ do not appear to be as sensitive to 3D $q_{\mu}$ structure as overtones modes $(n>0)$. However, fundamental modes should not be completely disregarded. Especially the low $l$ fundamentals $(l<7)$ appear isolated in the frequency spectrum, which means we can consider cross-coupling to other modes a secondary effect and measure them in self-coupling. In addition, fundamental modes have a large number of high-quality spectral data available, which makes them ideal to measure splitting functions. For example, mode ${ }_{0} S_{2}$ is a fundamental mode below $1 \mathrm{mHz}$, so we expect negligible sensitivity to 3D anelastic structure from it. On the other hand, the distinctive lower mantle sensitivity of mode ${ }_{0} S_{2}$ makes it ideal to observe lower mantle structure. Because of these reasons, we will still attempt to measure $3 \mathrm{D}$ anelastic structure from fundamental modes in this and the following chapter.

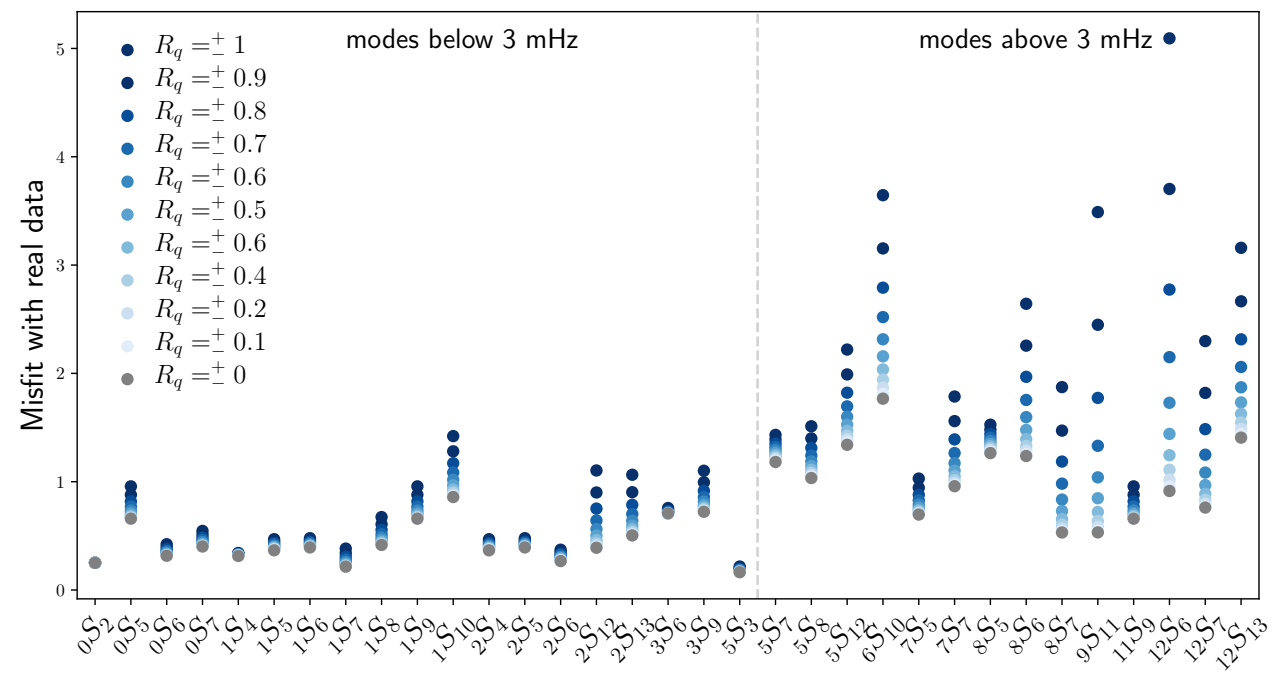

Figure 5.6: Misfit between observed spectral data and synthetic spectra calculated for model S20RTS combined with the 3D $q_{\mu}$ predictions of S20RTS scaled by the ratio $R_{q}$ $\left(-1 \leq R_{q} \leq 1\right)$. To calculate the misfit, we use the expanded vertical component dataset of Deuss et al. (2013). 


\subsubsection{Individual mode sensitivity to $3 \mathrm{D}$ mantle $q_{\mu}$}

In order to determine in more detail how sensitive normal modes are to $3 \mathrm{D}$ attenuation, we selected a subset of fundamental modes (e.g. ${ }_{0} S_{2},{ }_{0} S_{5},{ }_{0} S_{6},{ }_{0} S_{7}$ ), as well as first overtones (e.g. ${ }_{1} S_{6},{ }_{1} S_{7},{ }_{1} S_{8},{ }_{1} S_{9},{ }_{1} S_{10}$ ) and second overtones (e.g. ${ }_{2} S_{5},{ }_{2} S_{6},{ }_{2} S_{12}$, $\left.{ }_{2} S_{13}\right)$ to perform more detailed forward modelling of spectra and measure anelastic splitting functions from the synthetic spectra. With the exception of ${ }_{1} S_{6-}{ }_{2} S_{5}$ and ${ }_{1} S_{5-2} S_{4}$, we have selected these modes on the basis that they are isolated, so they can be measured in self-coupling, which simplifies the measurement procedure. In Fig. 5.6, we see that synthetic spectra generated by including elastic and anelastic splitting function coefficients in the matrix calculation, show the same qualitative behaviour for these modes as seen in the full-coupling synthetics discussed in Section 5.2.2. We have not compared the self-coupling and group-coupling approximation to the full-coupling approximation in a quantitatively manner, but we do not expect it to lead to a significant difference for the subset of modes we will focus on this and next chapter (Deuss and Woodhouse, 2001).

In order not to show too many individual figures for all these different modes, we concentrate on modes ${ }_{2} S_{12}$ and ${ }_{1} S_{10}$. We selected mode ${ }_{2} S_{12}$ because it is a typical upper mantle sensitive mode and mode ${ }_{1} S_{10}$ for its mainly lower mantle sensitivity. Although modes ${ }_{2} S_{12}$ and ${ }_{1} S_{10}$ are more weakly excited than other modes or groups of modes, there is a large amount of good quality data available for these modes,

S20RTS $c_{s t}$ predictions
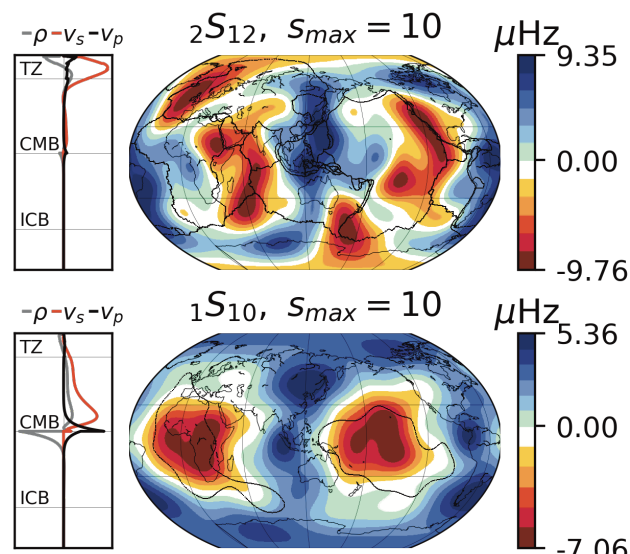

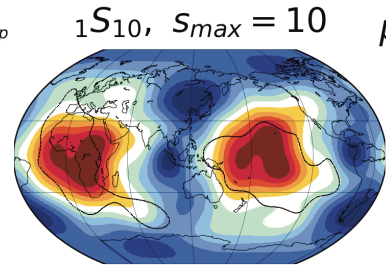

$$
{ }_{1} S_{10}, S_{\max }=10
$$
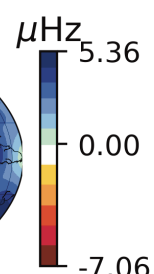

$-7.06$

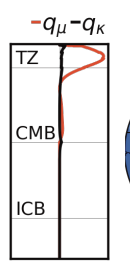

S20RTS $d_{s t}$ predictions $\delta q_{\mu}=-0.2 \delta v_{s} / v_{s}$
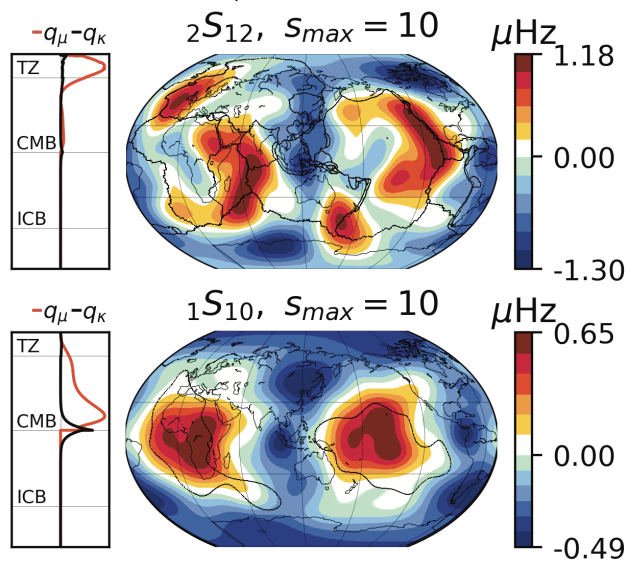

Figure 5.7: Elastic (real) splitting function predictions of S20RTS (left), together with the anelastic (imaginary) splitting function predictions of S20RTS scaled using $R_{q}=-0.2$ (right). Splitting functions are plotted together with their respective sensitivity kernels for $v_{s}$ and $q_{\mu}$ (red lines), $v_{p}$ and $q_{\kappa}$ (black lines) and $\rho$ (grey line). Top: ${ }_{2} S_{12}$, dominated by $v_{s}$ sensitivity to the upper mantle, together with the tectonic plate boundaries (Bird, 2003). Bottom: ${ }_{1} S_{10}$, dominated by $v_{s}$ sensitivity to the lower mantle, together with the $-0.1 \% v_{s}$ outline of the tomographic model SP12RTS at $2850 \mathrm{~km}$ (Koelemeijer et al., 2016), which contours the LLSVPs. 


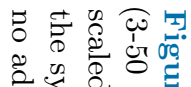

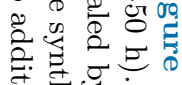
它 ज़

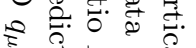

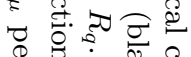
焉顿 导 क छ क्ष ठ 훙. ๙ิ

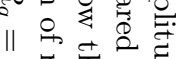

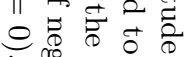

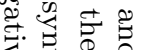

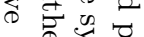
ऽָ

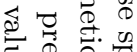

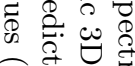
उ IV 1 ऊँ †守密. 붕용

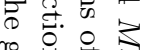
क्ष 4 话 ज. 팔. 능 ब

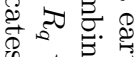
क串起 它志 此踏 总吉官 苛 $1 \wedge$ 记 $\tau=0$

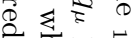
渮. 可 它吉节

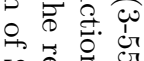
용 E용

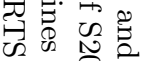

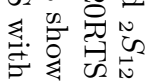
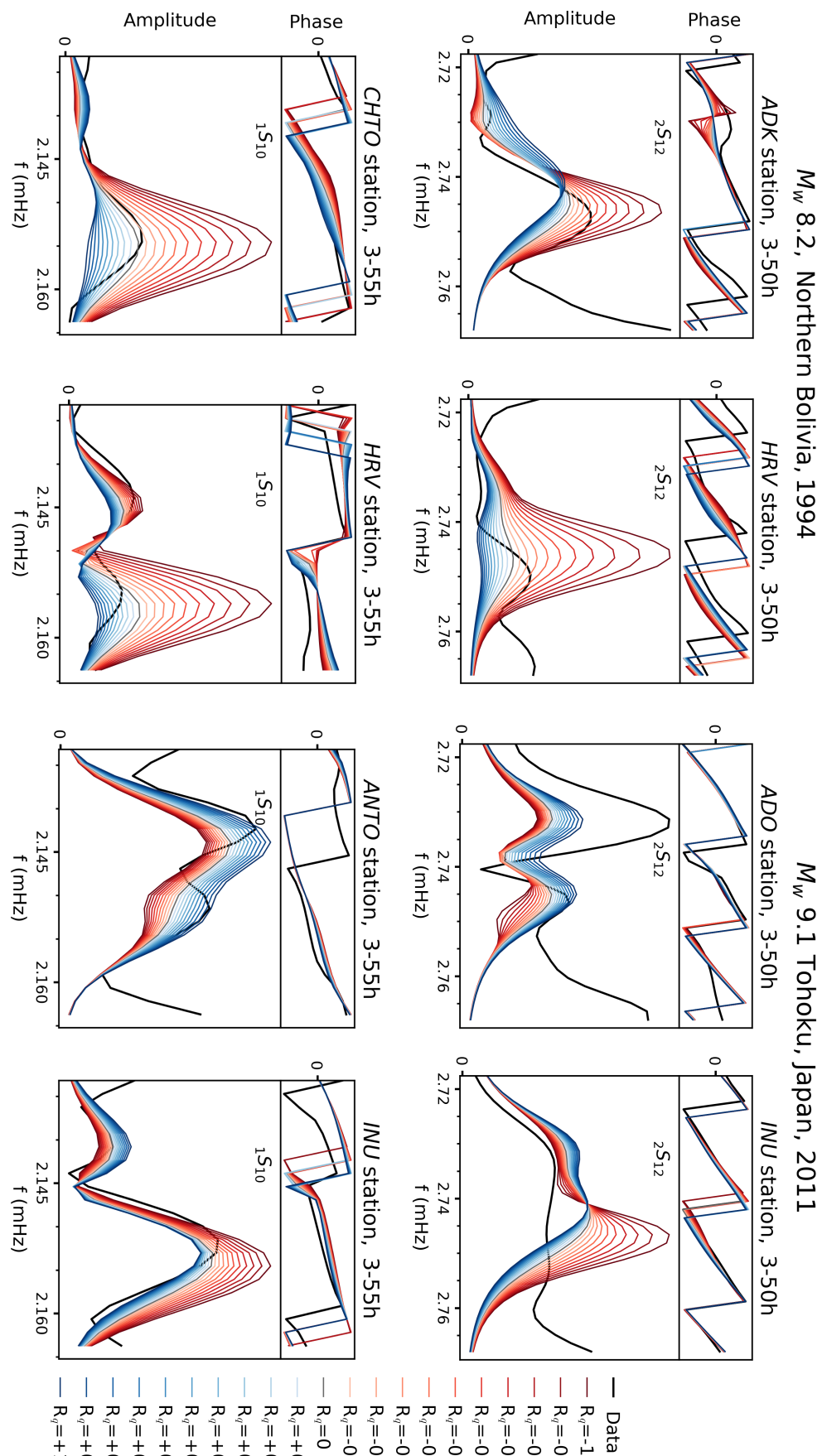

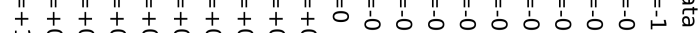




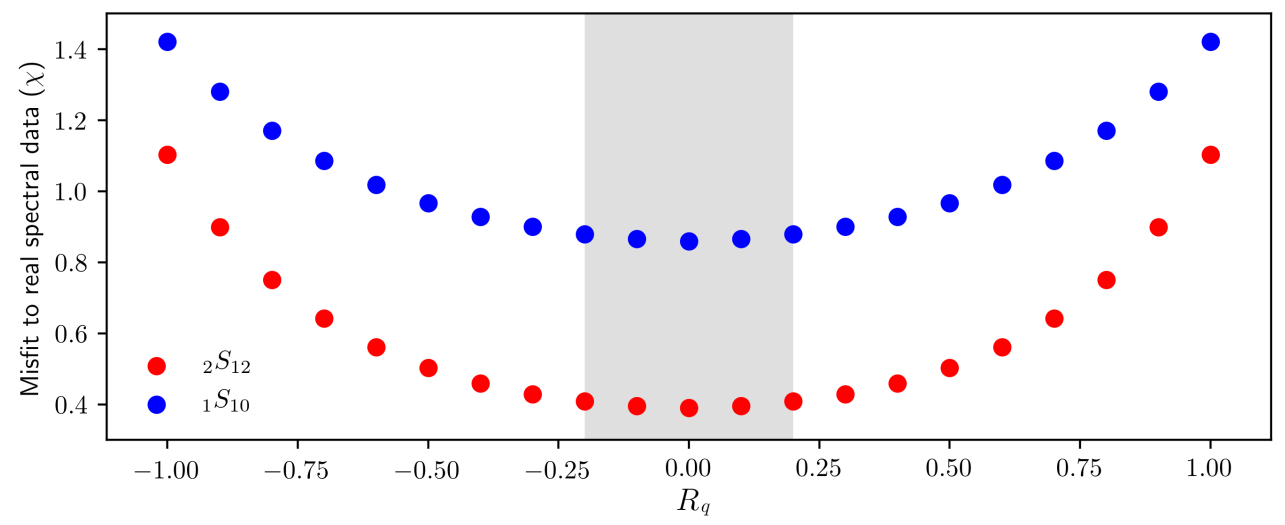

Figure 5.9: Misfit between observed spectral data and synthetic spectra calculated for model S20RTS combined with the 3D $q_{\mu}$ predictions of S20RTS scaled by the ratio $R_{q}$ $\left(-1 \leq R_{q} \leq 1\right)$. To calculate the misfit, we use the expanded dataset of Deuss et al. (2013): 2734 vertical component spectral segments from 101 events for ${ }_{2} S_{12}$; and 868 vertical component spectral segments from 78 events for ${ }_{1} S_{10}$.

which makes them well behaved when measuring elastic structure (e.g. Deuss et al., 2013). We expect that this behaviour will translate into our anelastic structure measurements.

In Fig. 5.7, we present the elastic splitting function predictions of S20RTS, and the anelastic splitting function predictions of our $q_{\mu}$ synthetic model obtained by scaling S20RTS using $R_{q}=-0.2$, for modes ${ }_{2} S_{12}$ and ${ }_{1} S_{10}$. When we use scaling $R_{q}=-0.2$, the predicted frequency variations of the anelastic splitting functions are on average 10 times smaller than those of the elastic splitting functions. This order of magnitude difference between our elastic and anelastic synthetic splitting function predictions gives us a sense of how large we might expect real 3D anelastic variations to be, once we perform measurements using real data. The fact that the anelastic splitting functions are much smaller than the elastic splitting functions, means that it might be challenging to measure anelastic splitting functions and that we have to develop a specific workflow to make it possible to measure the small anelastic splitting function coefficients in conjunction with the much larger elastic splitting function coefficients.

To further analyse the sensitivity of modes ${ }_{2} S_{12}$ and ${ }_{1} S_{10}$ to $3 \mathrm{D}$ attenuation perturbations, we create a range of different $3 \mathrm{D}$ synthetic attenuation models by varying $R_{q}$ from 1 to -1 , just as in Fig. 5.6. The synthetic $q_{\mu}$ model using $R_{q}=-1$ represents a one-to-one anti-correlation to model S20RTS, while the $q_{\mu}$ model for $R_{q}=1$ is completely correlated to S20RTS. $R_{q}=0$ represents a model with no 3D attenuation variations. These scaled $3 \mathrm{D}$ anelastic models are used in combination with the 3D elastic velocity variations of S20RTS. Fig. 5.8 shows the synthetic spectra calculated for these different $R_{q}$ values for modes ${ }_{2} S_{12}$ and ${ }_{1} S_{10}$. The presence of $3 \mathrm{D}$ attenuation variations mainly affects the spectral amplitude of the modes, while no significant changes can be appreciated in the spectral phase. The phase depends 
on the elastic structure only to first order, so we do not expect to see as strong an effect of $Q$ as in the amplitudes, where it is a leading order effect. Additionally, the phase is mostly affected by the shape of the anomalies; by scaling the shear wave velocity anomalies to obtain the anelastic anomalies we keep the shape the same, and therefore we indeed do not expect to see a large effect on the phase.

In order to quantify the overall effect, we calculate the misfit between the observed spectral data for real earthquakes and the synthetic spectra calculated using model S20RTS in combination with the 3D anelastic synthetic perturbations for a range of values for $R_{q}$. In Fig. 5.9, we observe that although the lowest misfit value is found at $R_{q}=0$ (only $3 \mathrm{D}$ elastic perturbations), $R_{q}$ values varying between -0.2 and 0.2 produce similar misfits. A similar behaviour was also seen in other modes we analysed. In particular, the misfit of ${ }_{2} S_{12}$ varies $4 \%$, from $R_{q}=0.2$ to $R_{q}=-0.2$, while the misfit of ${ }_{1} S_{10}$ varies $1.5 \%$. These small variations in misfit give us an indication that normal modes are dominated by elastic 3D perturbations, and that $3 \mathrm{D}$ anelastic perturbations, comparable in size to those measured in previous $3 \mathrm{D} q_{\mu}$ models (e.g. Dalton et al., 2008), will not alter the misfit to the real data massively. It is interesting to note that, although here we only show two modes, the misfit is lowest for $R_{q}=0$. Assuming that $3 \mathrm{D}$ attenuation would be the dominant effect necessary to fit the model predictions to the data, implies that there is no simple anti-correlation relationship between $v_{s}$ and $q_{\mu}$ in the whole mantle but that $R_{q}$ must be varying regionally. It is important to realise that this forward modelling assumes that the elastic model is perfect and that the $v_{p}$ and density scalings are correct, as well as the source moment tensor solutions, and that noise is not a significant factor. If this is not the case, it would mean S20RTS and the source solutions used here are not precise enough to allow to us 'see' the weaker $Q$ signal.

\subsection{Synthetic anelastic splitting function tests}

Our aim is to constrain $3 \mathrm{D}$ variations in mantle attenuation by measuring anelastic splitting functions. We have seen in the previous section that the anelastic splitting function coefficients $d_{s t}$ may be an order of magnitude smaller than the elastic splitting function coefficients $c_{s t}$. So, the question is if we can resolve the $d_{s t}$ at all, or if they are too small in comparison to the commonly measured $c_{s t}$ coefficients. In order to assess the potential of measuring $d_{s t}$ and the robustness of $d_{s t}$ measurements, we designed a set of tests. These tests evaluate our ability to recover $3 \mathrm{D}$ anelasticity splitting functions from synthetic data, as well as the dependence of the $d_{s t}$ values on potential uncertainties or systematic bias in the earthquake magnitudes. These tests were applied to all modes in Fig. 5.10. However, because all these modes showed the same response, we again use ${ }_{2} S_{12}$ and ${ }_{1} S_{10}$ as examples of typical upper mantle and lower mantle sensitive mode behaviour. Only in Section 5.3.2, we show the results of other modes in order to analyse the branch consistency of our tests.

The inversion scheme used is the iterated least-squares inversion (Tarantola and Valette, 1982), as explained in Chapter 2. To generate the synthetic models, we use the elastic splitting function predictions of $v_{s}$ mantle model S20RTS (Ritsema et al., 1999), while the synthetic anelastic splitting functions are again built by applying the scaling $\delta q_{\mu}=R_{q} \delta v_{s} / v_{s}$. We test three relationships types between elastic and 


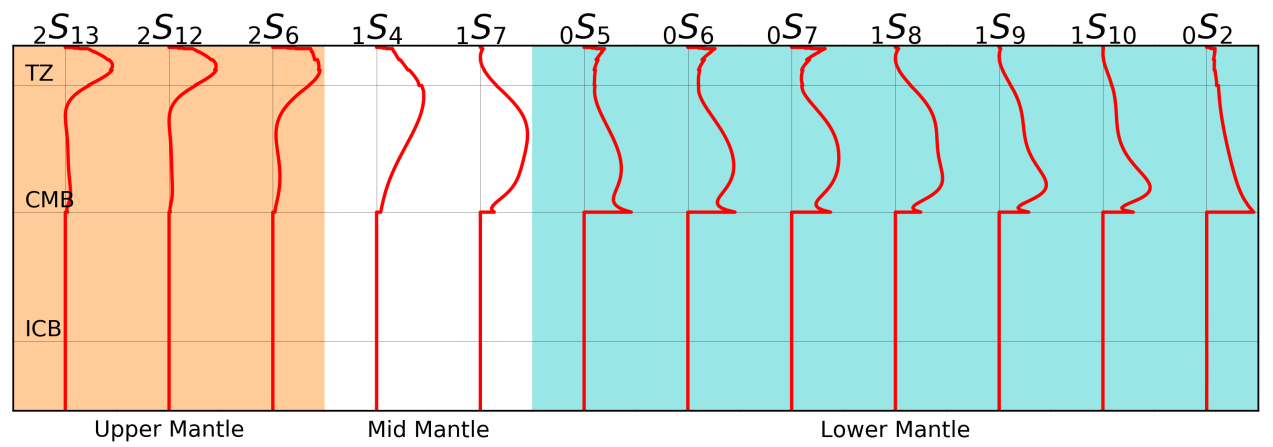

Figure 5.10: Sensitivity kernels as a function of depth for shear attenuation $q_{\mu}\left(\mu_{0} K_{\mu}\right)$, calculated for PREM (Dziewonski and Anderson, 1981). Modes are arranged and classified according to where they are most sensitive. The transition Zone (TZ), Core Mantle Boundary (CMB) and Inner Core Boundary (ICB) are marked in figure. All kernels are plotted for degree-zero. Higher structural degree kernels remain close to their respective degree-zero kernel, with the exception of mode ${ }_{0} S_{2}$, where the kernels flip sign for higher degree structure.

anelastic structure in the synthetic spectra: correlation $\left(R_{q}=0.2\right)$, anti-correlation $\left(R_{q}=-0.2\right)$ and zero $3 \mathrm{D}$ anelasticity $\left(R_{q}=0\right)$. As shown in Fig. 5.7, when the same $R_{q}$ value is applied to the whole mantle to generate a 3D $q_{\mu}$ model, the predicted anelastic splitting function frequency variations will be on average $40 \%$ larger for upper mantle sensitive modes (i.e. ${ }_{2} S_{12}$ ) than for lower mantle sensitive modes (i.e. $\left.{ }_{1} S_{10}\right)$.

When we use the scaling $R_{q}= \pm 0.2$ to obtain a $3 \mathrm{D} q_{\mu}$ model, the predicted synthetic anelastic splitting function coefficients $d_{s t}$ are on average 10 times smaller than the elastic splitting function coefficients $c_{s t}$ (Fig. 5.7). Because of this large difference in amplitude, we are not able to simultaneously measure the $c_{s t}$ and $d_{s t}$ splitting coefficients. To bypass this, we first measure the $c_{s t}$ coefficients on their own, using the same damping value for all elastic coefficients. In the second step, we use these $c_{s t}$ measurements as our starting model, and now measure the $c_{s t}$ and $d_{s t}$ coefficients simultaneously, allowing both to change in the inversion. All $d_{s t}$ measurements are started from PREM (Dziewonski and Anderson, 1981), which means zero values for the $d_{s t}$ coefficients in the starting model, and the same damping value is applied to all elastic and anelastic coefficients. In all tests using synthetic spectra, we are able to fully recover the input elastic splitting functions $c_{s t}$. Therefore, in the section below, we will focus on how well we can recover the input anelastic splitting function coefficients $d_{s t}$.

\subsubsection{Synthetic anelastic splitting function recovery}

In our first test, we evaluate the ability of our method to recover anelastic splitting functions from synthetic spectra, when the $d_{s t}$ amplitude is much smaller than the $c_{s t}$ amplitude. We use the elastic splitting function predictions of the $v_{s}$ mantle model S20RTS (Ritsema et al., 1999), together with three different 3D anelastic 


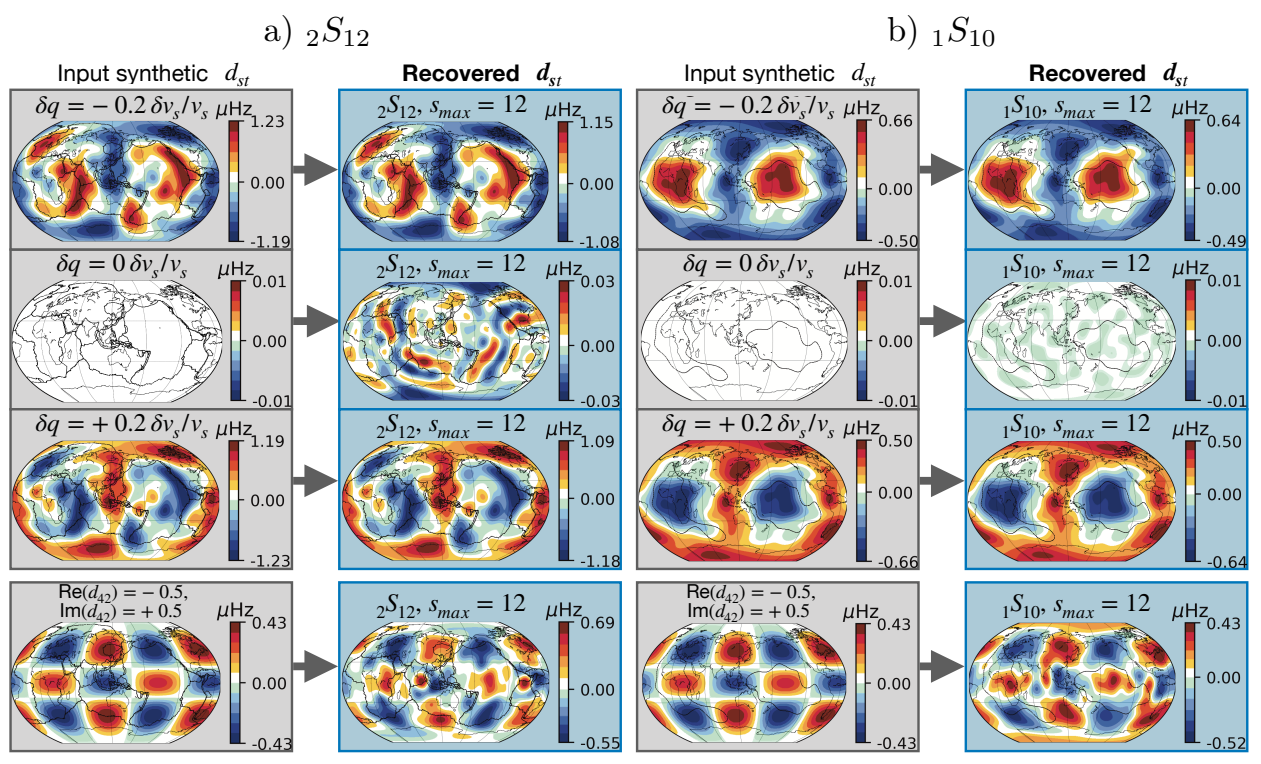

Figure 5.11: Anelastic splitting function $d_{s t}$ recovery tests using synthetic spectra for (a) upper mantle mode ${ }_{2} S_{12}$ (also shown are the tectonic plate boundaries of Bird, 2003), and (b) lower mantle mode ${ }_{1} S_{10}$ (also shown is the LLSVP outline of the tomographic model SP12RTS at $2850 \mathrm{~km}$ of Koelemeijer et al., 2016). The grey panels show the input synthetic $d_{s t}$, with the title of each sub-figure outlining the characteristics of each synthetic input. These synthetic input $d_{s t}$ were obtained by scaling the elastic model S20RTS (Ritsema et al., 1999) using $\delta q=R_{q} \delta v_{s} / v_{s}$ and are then used to calculate synthetic spectra from which the recovered $d_{s t}$ were measured. The blue panels show the recovered $d_{s t}$ together with $s_{\max }$. All $d_{s t}$ synthetic measurements are showed up-to the $s_{\max }$ used in their inversion.

splitting functions obtained by scaling $R_{q}=-0.2,0,0.2$. We follow the inversion steps previously outlined, by first measuring only the $c_{s t}$ values and then using these as the starting model in the joint inversion for both $c_{s t}$ and $d_{s t}$.

Fig. 5.11, shows both the input $d_{s t}$ used for calculating the synthetic spectra and the recovered $d_{s t}$ in the splitting function measurement for both the more strongly attenuating upper mantle sensitive mode ${ }_{2} S_{12}$ and the more weakly attenuating lower mantle sensitive mode ${ }_{1} S_{10}$. In all cases we are able to recover both the input elastic (not shown) and input anelastic splitting functions. In the particular case of the zero 3D anelastic input model (i.e. $R_{q}=0$ ), our recovered measured synthetic $d_{s t}$ coefficients are non-zero, but negligible (below $0.03 \mu \mathrm{Hz}$ for ${ }_{2} S_{12}$ and even smaller for ${ }_{1} S_{10}$ ). Furthermore, the amplitudes of the recovered anelastic splitting functions are only slightly smaller (less than 10\%) than the amplitudes of the input anelastic splitting functions. Thus, the effect of the damping in the inversion on reducing (and potentially underestimating) the anomalies of the recovered splitting functions is minimal.

In addition, we also tested a 3D input anelastic splitting function that holds no correlation to the elastic splitting function predictions of S20RTS. To create this input 3D anelastic splitting function, we set all $d_{s t}$ coefficients to zero, except for 
a) Location independent $M_{0}$ perturbations

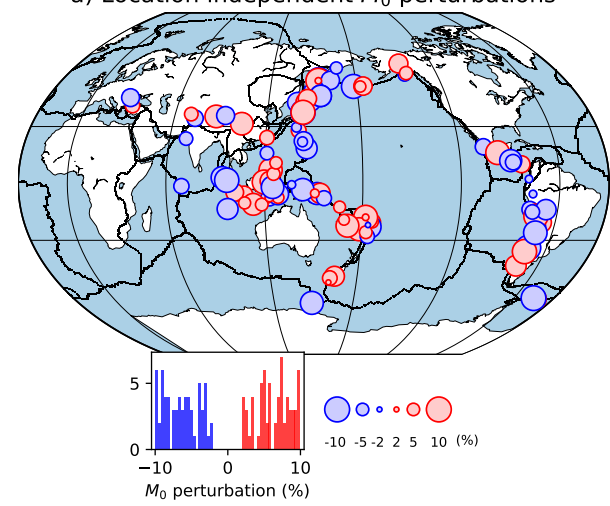

b) Location dependent $M_{0}$ perturbations

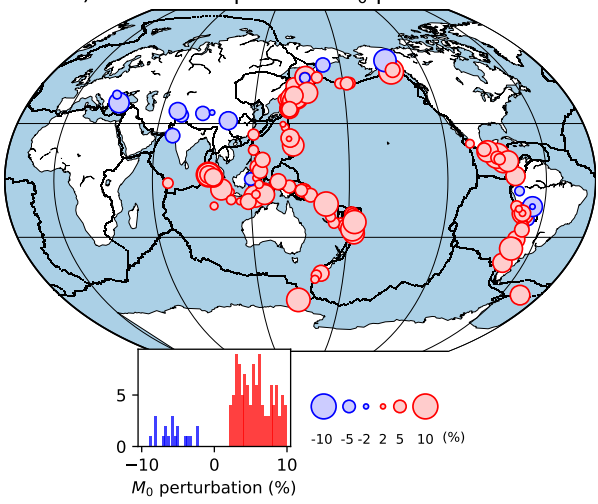

Figure 5.12: Map showing the scalar moment $M_{0}$ perturbations for the events used to measure the splitting functions. (a) Independent of earthquake location and (b) dependent of earthquake location, where events are classified by their location in oceanic ridges or trenches $\left(M_{0}\right.$ increase), continents $\left(M_{0}\right.$ decrease) or ocean floors $\left(M_{0}\right.$ increase or increase). The size of the circles indicates the size of the $M_{0}$ perturbation, which varies between $|2 \%| \leq M_{0} \leq|10 \%|$. Blue circles represent a decrease in $M_{0}$ and red circles an increase. We use a uniform distribution to randomly assign the $M_{0}$ variations (bottom left in each panel).

$\operatorname{Re}\left(d_{42}\right)=-0.5$ and $\operatorname{Im}\left(d_{42}\right)=0.5$. We again are able to recover both the elastic and anelastic input splitting functions (see Fig. 5.11 bottom panels).

\subsection{2 $M_{0}$ variation effect on anelastic splitting functions}

In our second test, we investigate the effect of the earthquake magnitude on the anelastic splitting function measurements. From previous studies (Hjörleifsdóttir and Ekström, 2010; Karaoğlu and Romanowicz, 2018), we know that unaccounted 3D mantle elastic heterogeneity may alter the scalar moment $M_{0}$ of the moment tensor solution by up-to $10 \%$, when compared to the solutions published in the Global Centroid Moment Tensor database (www.globalcmt.org). We will first introduce $M_{0}$ variations that are random and do not take into account the specific location of the earthquakes (Fig. 5.12a). Next, we will also explore earthquake location dependence (Fig. 5.12b), because we know that location plays a role in $M_{0}$ magnitude variations, with ridges and trenches experiencing an increase in magnitude and continents a decrease (Karaoğlu and Romanowicz, 2018). This may be especially important in our study, where the vast majority of the earthquakes are located in ridge and trench areas (Fig. 2.4). The 2004 Sumatra earthquake was excluded from our measurements and tests given its complicated source and associated uncertainties.

We vary $M_{0}$ of all earthquakes used in this study randomly between $\pm 2 \%$ and $\pm 10 \%$ for each earthquake when calculating the synthetic spectra, which is much larger than the reported uncertainties. We again include the previously mentioned zero 3D anelasticity model, as well as a correlated and an anti-correlated elastic and anelastic splitting functions, in calculating the synthetic spectra. We then use 

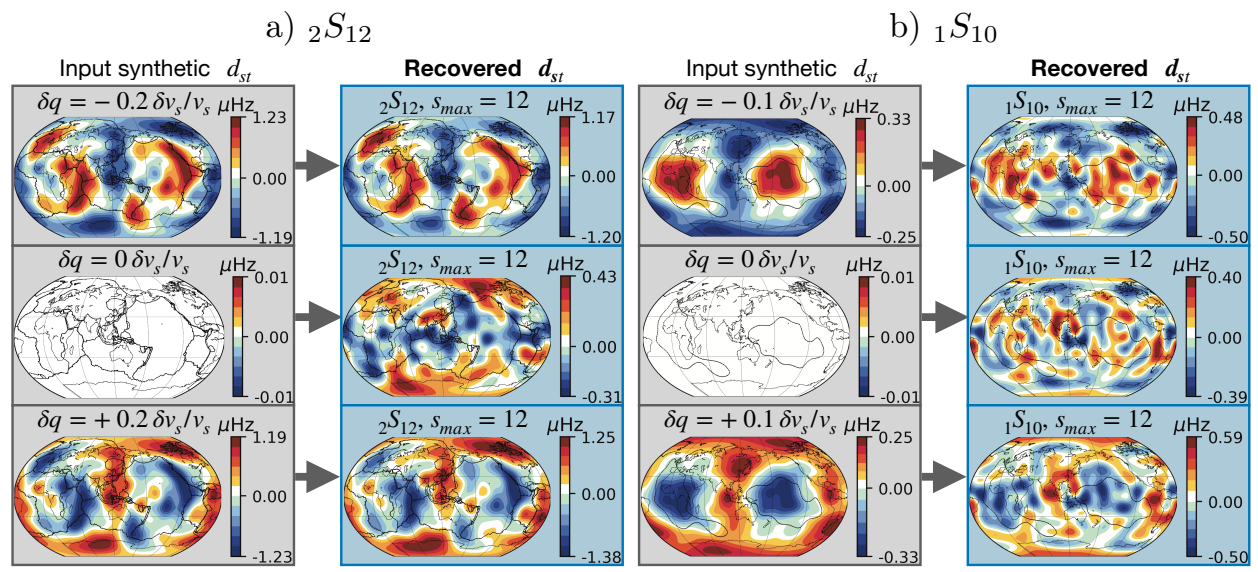

Figure 5.13: Similar to Fig. 5.11, but now showing anelastic splitting function $d_{s t}$ recovery tests including $M_{0}$ variations independent of earthquake location (e.g. Fig. 5.12a). Recovery tests shown for (a) upper mantle sensitive mode ${ }_{2} S_{12}$ and (b) lower mantle sensitive mode ${ }_{1} S_{10}$. The grey panels show the input synthetic $d_{s t}$; the blue panels show the recovered $d_{s t}$.

the unaltered $M_{0}$ values when measuring the elastic and anelastic splitting function coefficients. We find that when 3D anelastic structure $\left(R_{q} \geq|0.1|\right)$ is present, we are able to recover the input $d_{s t}$. This is especially noticeable for lower mantle mode ${ }_{1} S_{10}$ (Fig. 5.13b), for which the input $d_{s t}$ is recovered even when using a weaker $3 \mathrm{D} q_{\mu}$ model $\left(R_{q}= \pm 0.1\right)$ than upper mantle mode ${ }_{2} S_{12}\left(R_{q}= \pm 0.2\right.$, Fig. 5.13a). At the same time, it is important to note that in our tests with zero 3D anelasticity input $\left(R_{q}=0\right)$, we retrieve $d_{s t}$ structure that is non-zero, the shape of which depends on the particular combination of $M_{0}$ variations used in the test. These 'ghost' structures appear in a branch consistent manner (i.e. modes with the same $n$ show similar patterns), which we will explore in more detail using location dependent $M_{0}$ variations.

To evaluate location dependent $M_{0}$ variations, we include a random increase in $M_{0}$ magnitude for earthquakes located near oceanic ridges or trenches and a random $M_{0}$ decrease for continental earthquakes, while oceanic floor earthquakes are given either a random increase or decrease in $M_{0}$ magnitude (e.g. Fig. 5.12b). When taking into account location dependent $M_{0}$ perturbations in combination with a zero 3D $q_{\mu}$ input model, we again measure 'ghost' structures in a branch consistent manner (Figs. 5.14-5.15). The size of the $d_{s t}$ ghost patterns is small, in most cases less than $5 \%$ of the $c_{s t}$ values. All evaluated modes $(n=0,1,2)$ show $3 \mathrm{D}$ anelastic 'ghost' $d_{s t}$ structure which appears to be more correlated than anti-correlated to 3D elastic $c_{s t}$ structure (Figs. 5.16-5.18). For the fundamental and first overtones, the correlation is mainly visible along the equator, where positive frequency anomalies are seen below Indonesia and Central America in both $c_{s t}$ and $d_{s t}$ degree two structure $(s=2)$ (Figs. 5.16-5.17). In the second overtone branch the correlation becomes more obvious, with negative frequency anomalies visible in the Eastern part of the central Pacific and eastwards of Africa and positive frequency anomalies in Indonesia and west of Africa in both $c_{s t}$ and $d_{s t}$ structure (Fig. 5.18). This pattern changes 

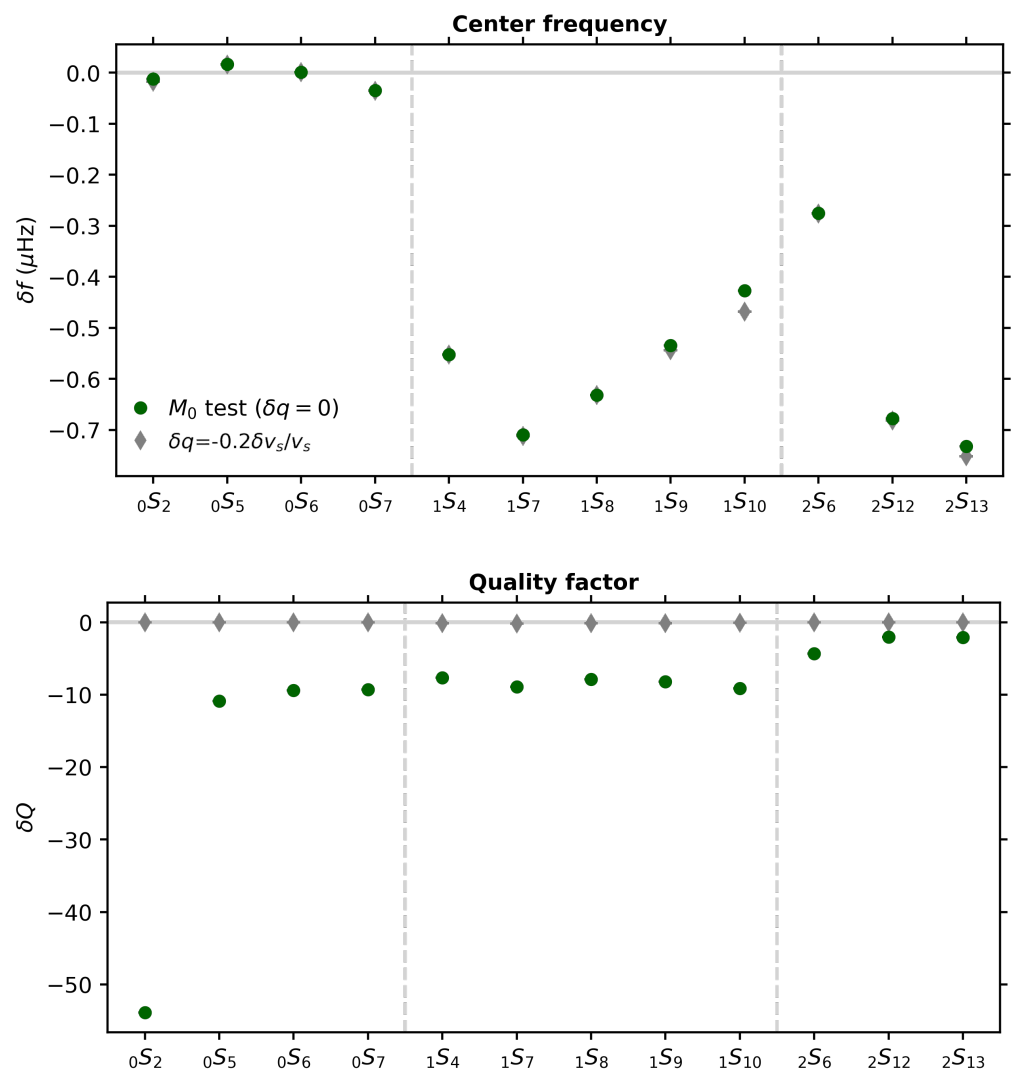

Figure 5.14: Branch plot comparison with respect to PREM (grey solid line) of centre frequencies $f_{c}(\mu \mathrm{Hz})$ and $Q$ values. These parameters are plotted for the recovered synthetic inversion (green), and the elastic predictions of S20RTS (Ritsema et al., 1999) combined with a 3D $q_{\mu}$ global model obtained by scaling S20RTS with $R_{q}=-0.2$ (grey). The input synthetic coefficients were calculated using the elastic 3D $v_{s}$ variations from S20RTS combined with zero $3 \mathrm{D} q_{\mu}$ variations and scalar moment $M_{0}$ perturbations dependent on earthquake location (Fig. 5.12b). This means all input synthetic anelastic coefficients are zero and all input elastic coefficients correspond to the predictions of S20RTS.

and shows more complexity at degree four and six, but stays branch consistent. In the case of $n=2$ modes sensitive to the upper mantle, correlation between 3D elastic $c_{s t}$ and anelastic $d_{s t}$ structure is the opposite of what we expect based on existing upper mantle 3D attenuation models (e.g. Dalton et al., 2008). This means that location dependent $M_{0}$ variations are expected to lower the measured upper mantle anelastic seismic signal in the $2^{\text {nd }}$ overtone modes.

Only mode ${ }_{0} S_{2}$ shows a different behaviour. It has a negative $v_{s}$ and $q_{\mu}$ sensitivity kernel for degree-two structure, so its frequency variations should be interpreted in the opposite manner as other modes, i.e. high frequencies relate to low attenuation. In our test, we find that ${ }_{0} S_{2}$ shows anti-correlation between elastic $c_{s t}$ and anelastic 


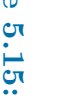

ఊ్ర

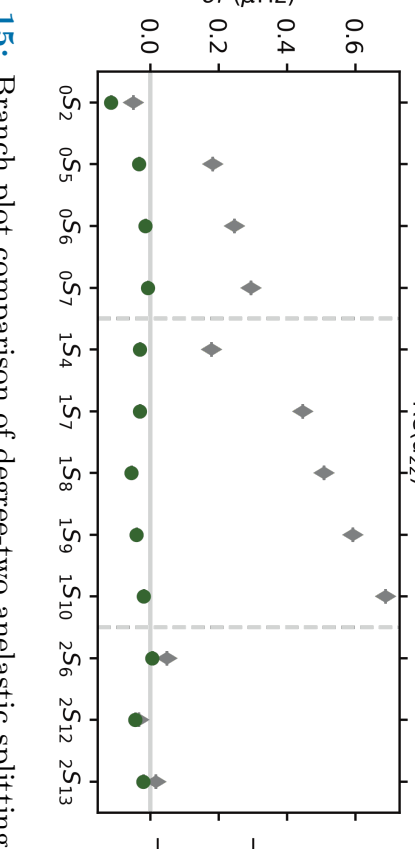

$(\mu \mathrm{Hz})$

$\delta f(\mu \mathrm{Hz})$

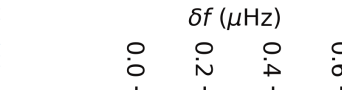
1
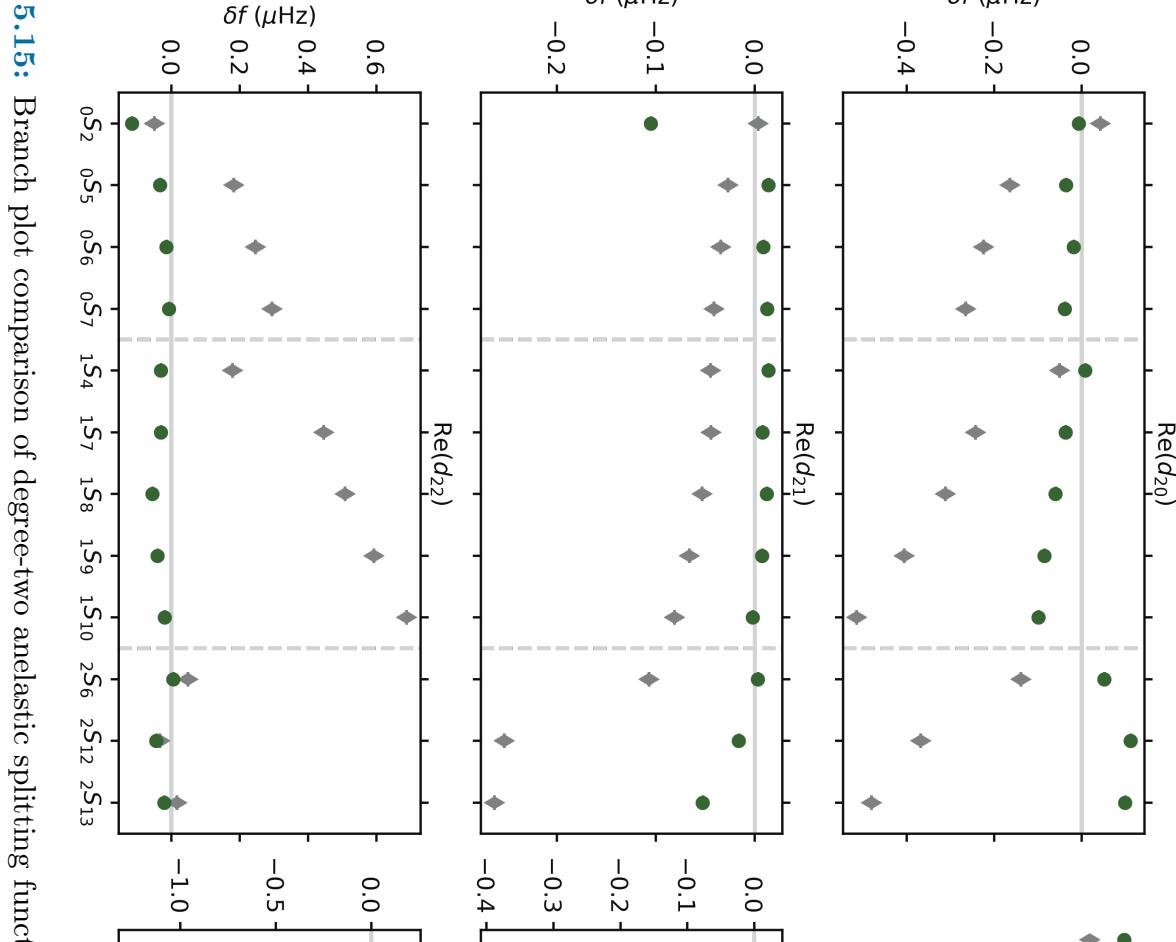

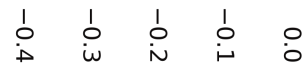
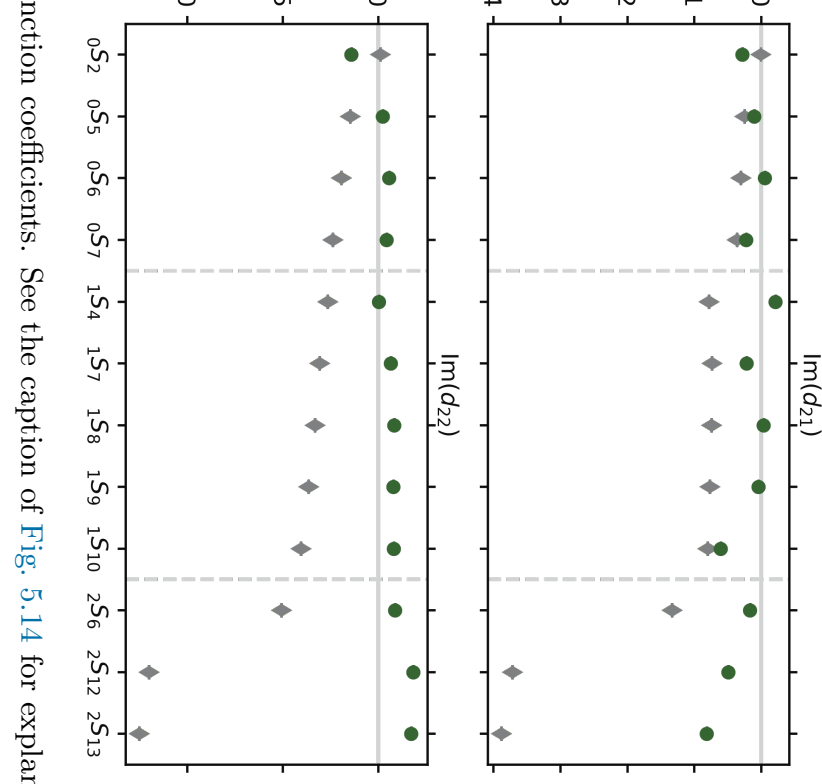

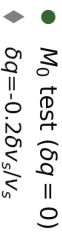

$\stackrel{\rightleftarrows}{\circ}$ 


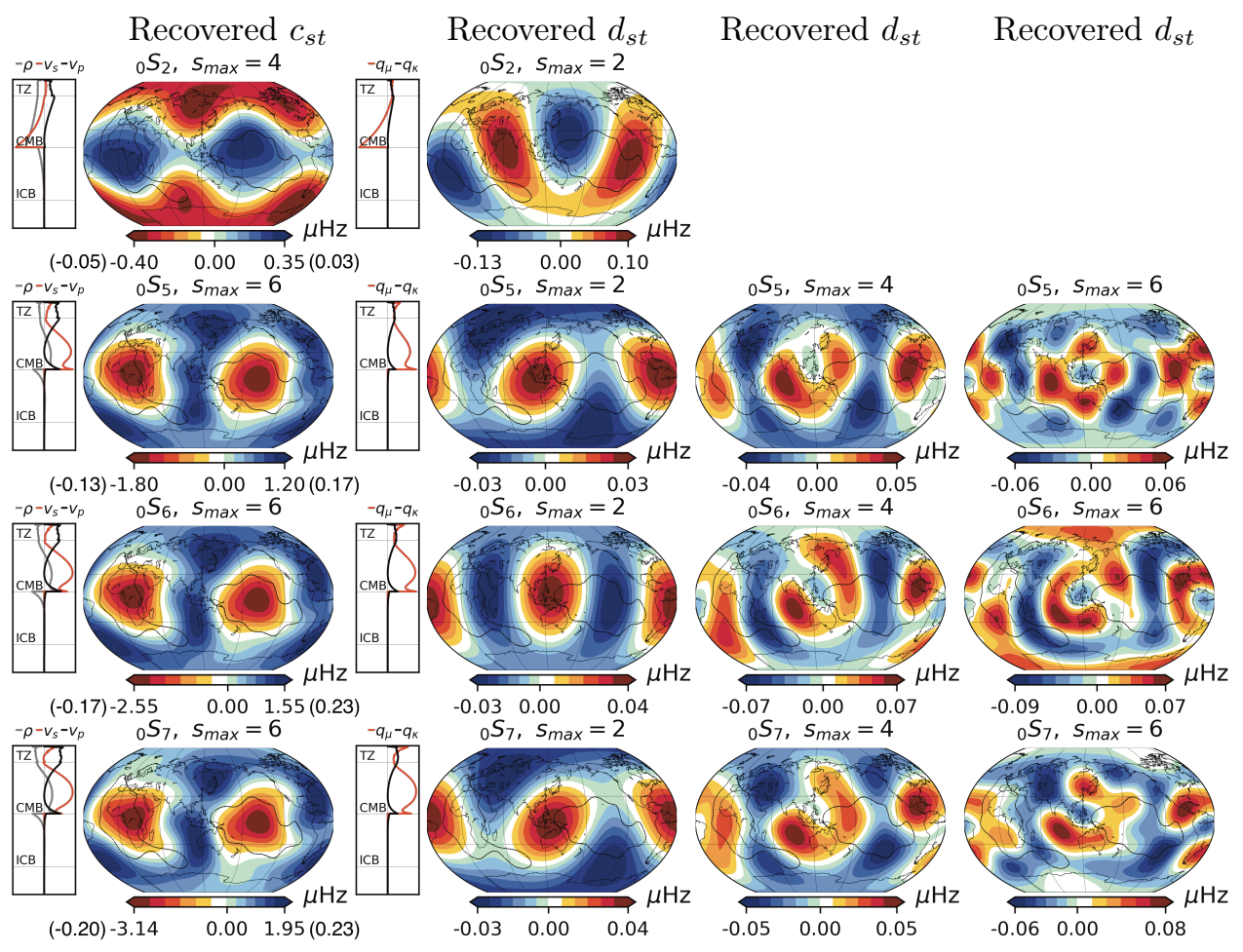

Figure 5.16: Recovered elastic and anelastic splitting functions for fundamental modes ${ }_{0} S_{l}$ measured from synthetic input spectra, which include scalar moment $M_{0}$ perturbations dependent on earthquake location (Fig. 5.12b). The input splitting functions have zero 3D anelastic variations and are calculated using the predictions of S20RTS. The input $c_{s t}$ (not shown) is fully recovered (left column). Even though the input $d_{s t}$ is zero, we recover 3D anelastic 'ghost' structure as a result of the $M_{0}$ perturbations. In parenthesis, next to the frequency range of the recovered $c_{s t}$, we have added the frequency range of the anelastic predictions of S20RTS scaled by $R_{q}=-0.2$.

'ghost' $d_{s t}$ structure, with positive frequency anomalies visible in the $c_{s t}$ and negative frequency anomalies in the $d_{s t}$ in the two LLSVPs regions. Thus, ${ }_{0} S_{2}$ is not consistent with other fundamental lower mantle sensitive modes. Furthermore, the measured 'ghost signal' of mode ${ }_{0} S_{2}$ is significantly larger than for other the fundamental modes, which is probably caused by the smaller data set available for ${ }_{0} S_{2}$ leading to a significant null space.

With the exception of mode ${ }_{0} S_{2}$, all other lower mantle sensitive fundamental modes $(n=0)$ show small 3D anelastic 'ghost' frequency variations of up-to 0.04 $\mu \mathrm{Hz}$ for degrees two to six (Fig. 5.16). Lower mantle sensitive modes with $n=1$ show slightly larger 3D anelastic 'ghost' frequency variations of up-to $0.08 \mu \mathrm{Hz}$ for degree-two (Fig. 5.17), and increasing systematically for degrees four and six (maximum $0.19 \mu \mathrm{Hz}$ ). The same behaviour can be seen in upper mantle sensitive modes with $n=2$, for which the 3D anelastic 'ghost' frequency variations increase to 


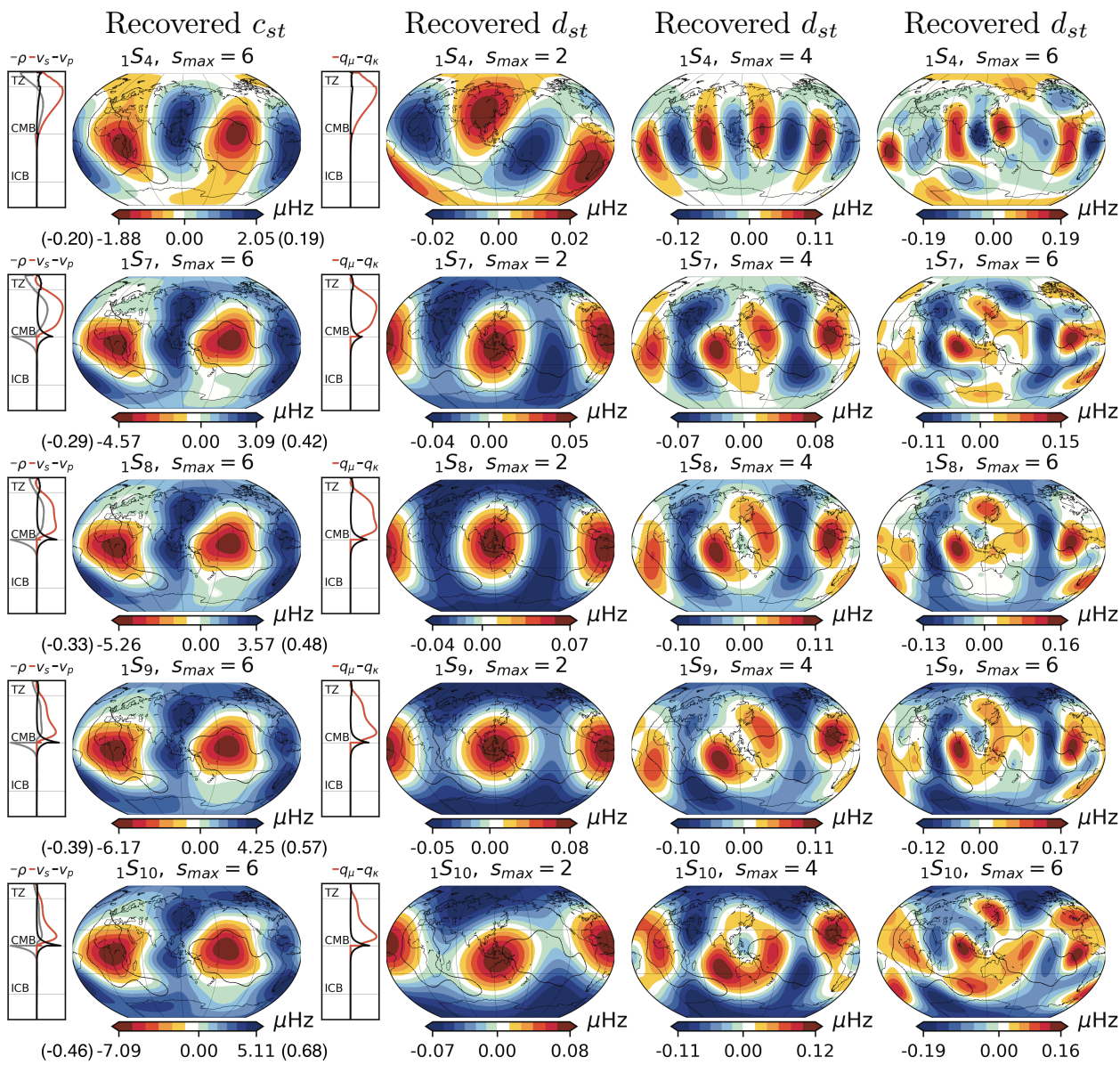

Figure 5.17: Recovered elastic and anelastic splitting functions for modes ${ }_{1} S_{l}$ measured from synthetic spectra, which include scalar moment $M_{0}$ perturbations dependent on earthquake location. See the caption of Fig. 5.16 for explanation.

up-to $0.34 \mu \mathrm{Hz}$ at degree six (Fig. 5.18). We will need to keep the size of these 'ghost' patterns, due to unaccounted $M_{0}$ variations, in mind when making $d_{s t}$ measurements using real data in the next chapter.

\subsection{Conclusion}

We have shown that spectra of mantle sensitive modes show observable differences when $3 \mathrm{D}$ variations in mantle attenuation are included. We also performed tests to evaluate the feasibility of jointly measuring elastic and anelastic splitting functions from normal mode spectra. We found that we were able to recover input synthetic anelastic splitting functions, even when they were an order of magnitude smaller than the elastic splitting functions. We also found that the 'ghost' anelastic splitting 


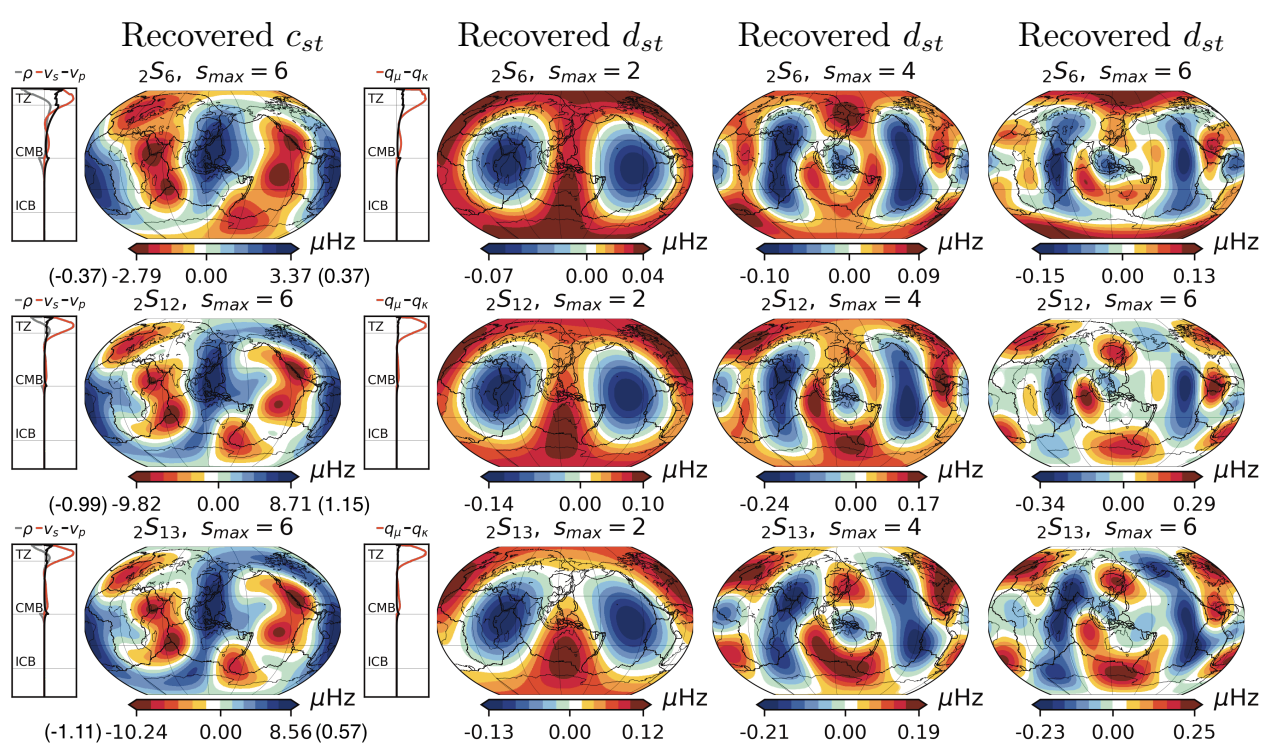

Figure 5.18: Recovered elastic and anelastic splitting functions for modes ${ }_{2} S_{l}$ measured from synthetic spectra, which include scalar moment $M_{0}$ perturbations dependent on earthquake location. Tectonic plate boundaries (Bird, 2003) are included for comparison. See the caption of Fig. 5.16 for explanation.

functions, which are the result of unaccounted moment magnitude variations when no $3 \mathrm{D}$ attenuation structure is present, might only be a problem for lower mantle modes. This is the case when the size of lower mantle anelastic splitting functions is $50 \%$ smaller than the scaled amplitude signal of expected upper mantle attenuation $\left(\delta q_{\mu}<|0.1| \delta v_{s} / v_{s}\right)$. So, we will be able to measure lower mantle anelastic splitting functions, even when taking scalar moment variations $M_{0}$ in the moment tensor solution into account, as long as the size of lower mantle anelastic splitting functions is at least $50 \%$ of the scaled amplitude signal of expected upper mantle attenuation. We will use these test results to validate the anelastic splitting function observations using real data outlined in the following chapter.

Additionally, because there is no available global 3D mantle attenuation model, we will use the scaled synthetic $3 \mathrm{D} q_{\mu}$ mantle model as a means to compare and validate our 3D anelastic observations. In order to obtain a scaled 3D $q_{\mu}$ mantle model anti-correlated to $3 \mathrm{D} v_{s}$, we will use the scaling $R_{q}=-0.2$, which we calculated based on the average relationship between the 3D $q_{\mu}$ in model QRFSI12 (Dalton et al., 2008) and the 3D $v_{s}$ in model S20RTS (Ritsema et al., 1999). 


\section{A Appendix: $\boldsymbol{R}_{q}$ ratio variations}
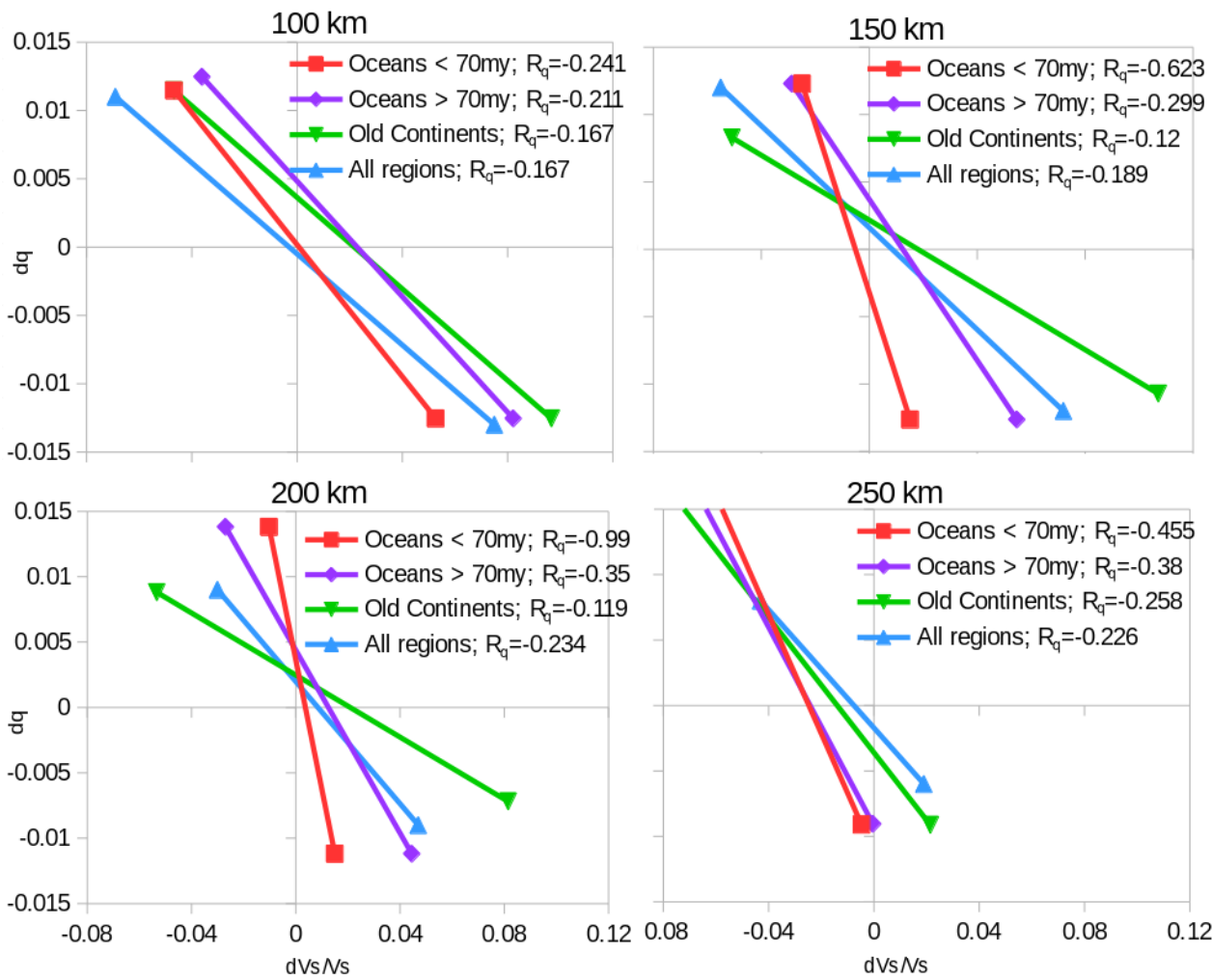

Figure 5.A.1: Modified from Fig. 7 of Dalton et al. (2009). Here, the absolute values of $q$ and $v_{s}$ were converted to $\delta q$ and $\delta v_{s} / v_{s}$ at different depths using PREM (Dziewonski and Anderson, 1981) as the 1D reference model. The figure shows the best linear fit obtained by Dalton et al. (2009), between $\delta q$ and $\delta v_{s} / v_{s}$ in each tectonic region of QRFSI12 (Dalton et al., 2008) and S362ANI (Kustowski et al., 2008). 
a)

QRFSI12 relationship to S20RTS
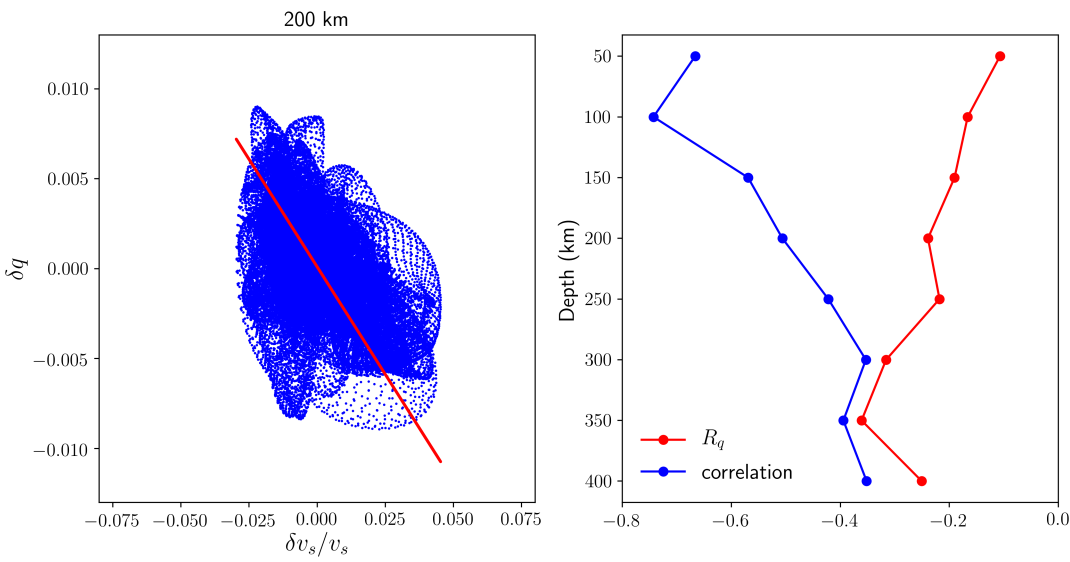

b)

QRFSI12 relationship to S362ANI
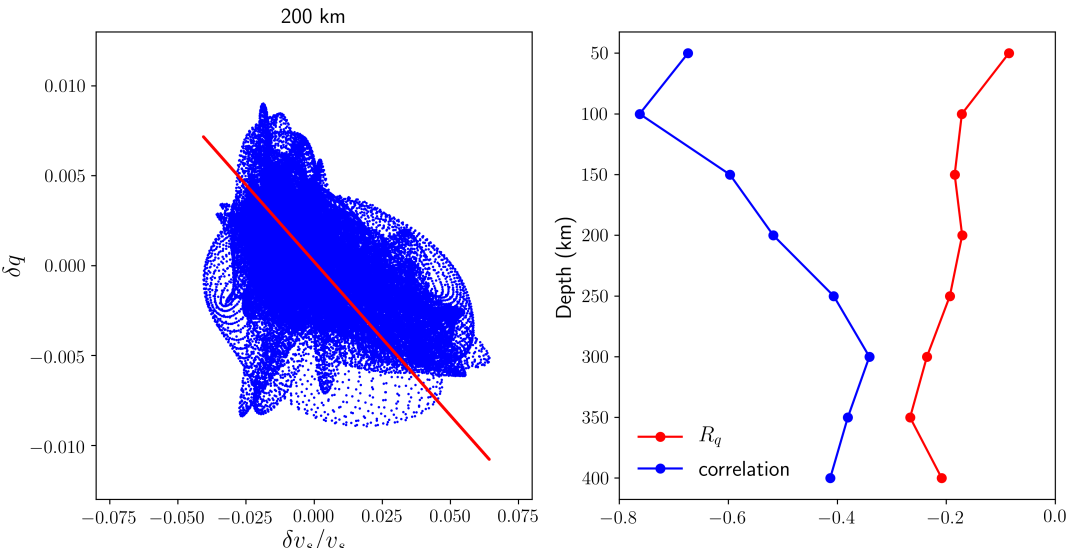

c)

QRFSI12 relationship to SEMum2
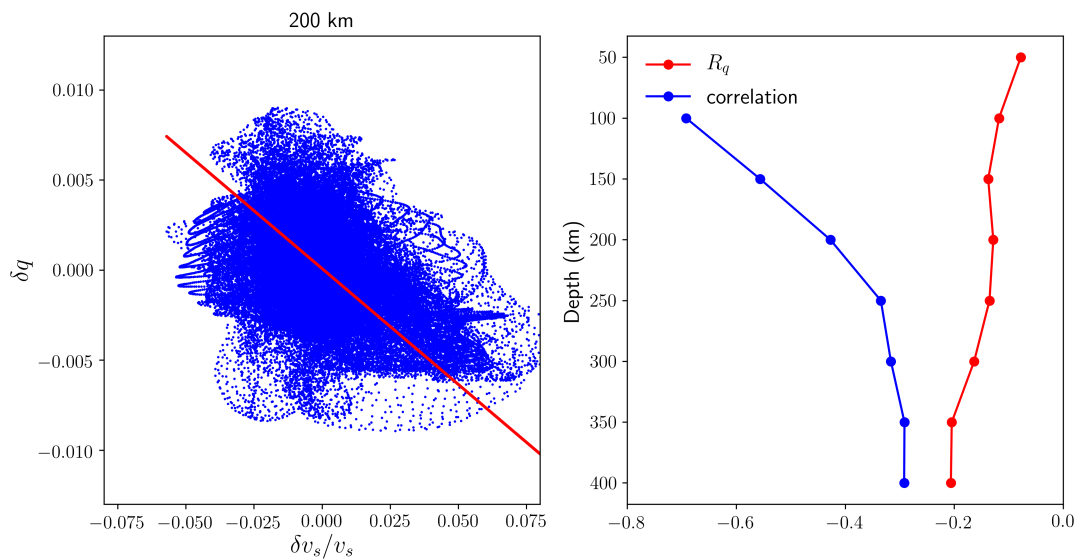

Figure 5.A.2: (left column) Each blue dot represents an equidistant latitude and longitude location in QRFSI12 $(\delta q)$ and three 3D mantle models $\left(\delta v_{s} / v_{s}\right)$ at $200 \mathrm{~km}$ depth. The red line represents the linear fit between $\delta q$ and $\delta v_{s} / v_{s}$. (right column) $R_{q}$ ratio (red line) and correlation (blue line) as a function of depth, illustrating the relationship between QRFSI12 and three 3D mantle models: (a) S20RTS (Ritsema et al., 1999); (b) S362ANI+M (Moulik and Ekström, 2014); and (c) SEMum2 (French et al., 2013) 

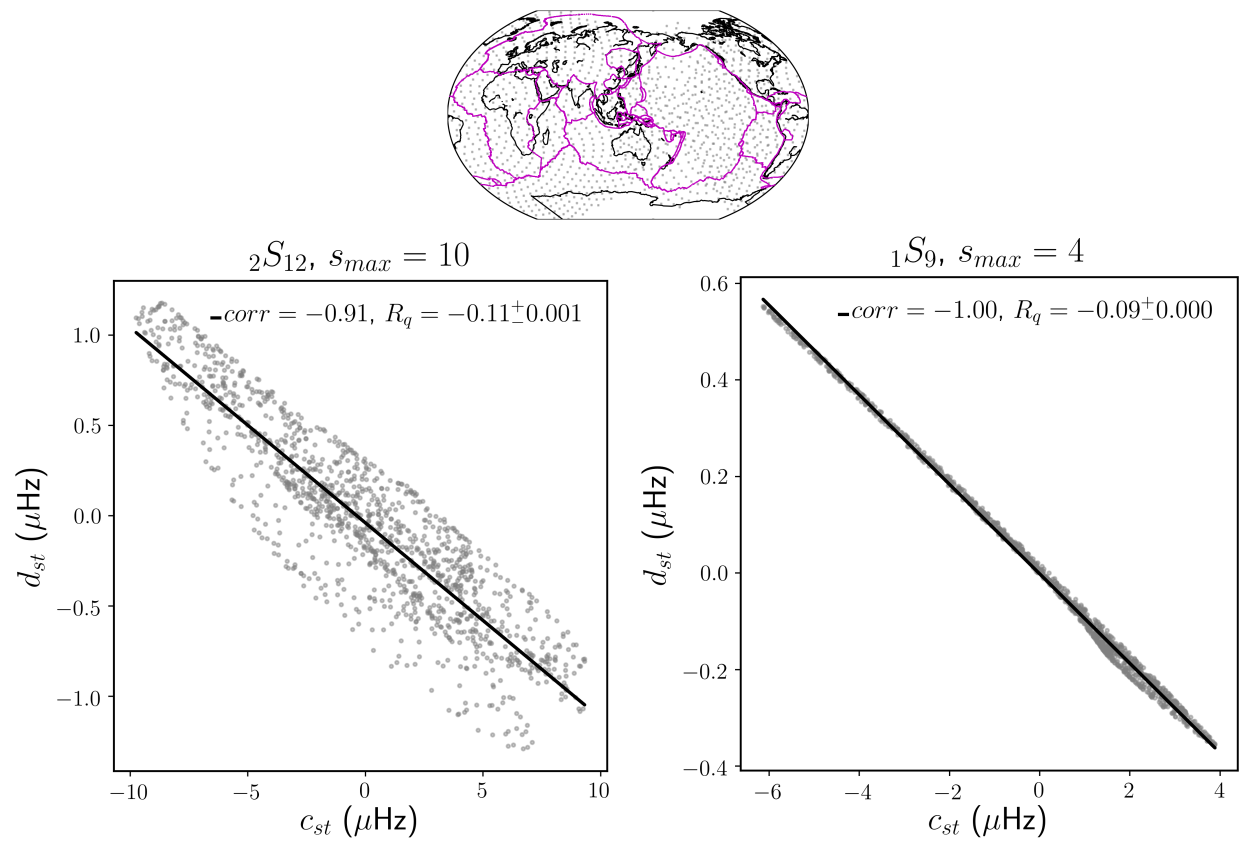

Figure 5.A.3: $c_{s t}$ versus $d_{s t}$ splitting function map values compared for upper mantle sensitive mode ${ }_{2} S_{12}$ and lower mantle sensitive mode ${ }_{1} S_{9}$. The splitting functions maps are obtained using the $c_{s t}$ predictions of S20RTS (Ritsema et al., 1999) and the $d_{s t}$ predictions of S20RTS using the scaling relationship $\delta q_{\mu}=-0.2 \delta v_{s} / v_{s}$ in the mantle. Each grey point (1300) represents an equidistant latitude and longitude location in the $c_{s t}$ and $d_{s t}$ splitting function maps. A simple linear regression is calculated, where corr is the correlation and $R_{q}$ is the slope. 
S20RTS $c_{s t}$ predictions
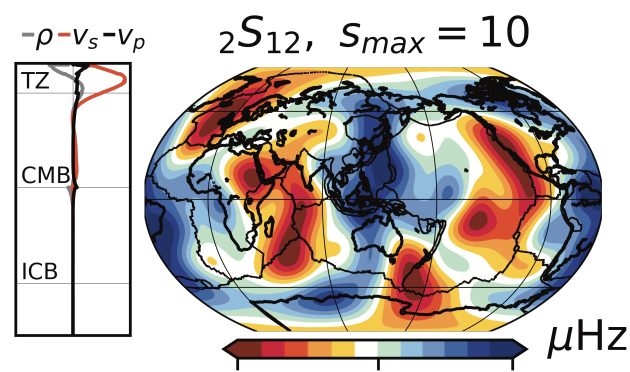

$\begin{array}{lll}-9.81 & 0.00 & 9.35\end{array}$

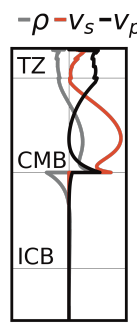

${ }_{0} S_{6}, S_{\max }=10$

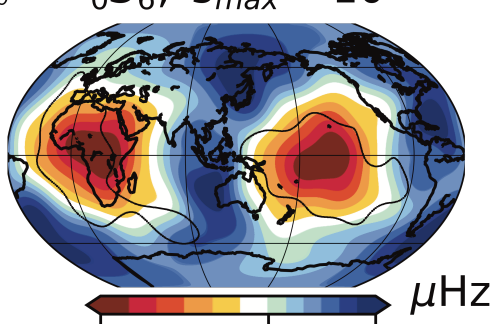

$-2.51$

0.001 .61
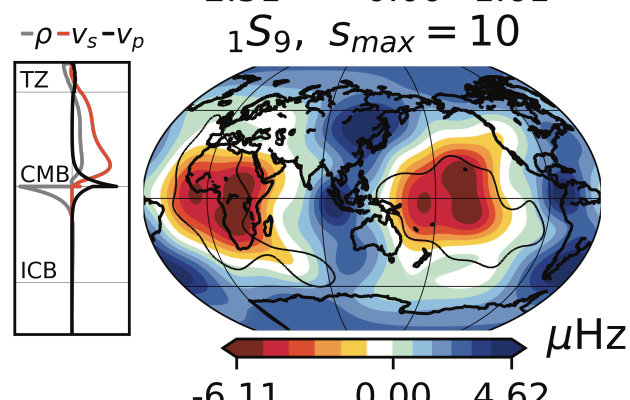

S20RTS $d_{s t}$ predictions

Upper mantle: $\delta q_{\mu}=-0.2 \delta v_{s} / v_{s}$

Lower mantle: $\delta q_{\mu}=+0.2 \delta v_{s} / v_{s}$

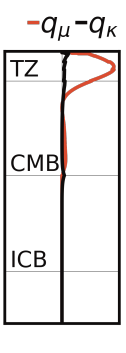

${ }_{2} S_{12}, S_{\max }=10$

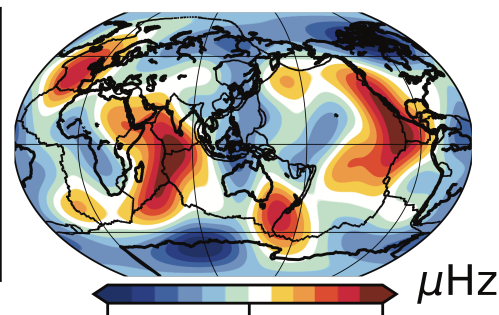

$\begin{array}{lll}-1.23 & 0.00 \quad 1.16\end{array}$

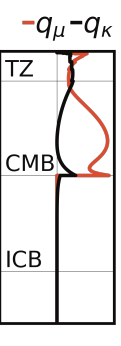

${ }_{0} S_{6}, S_{\max }=10$

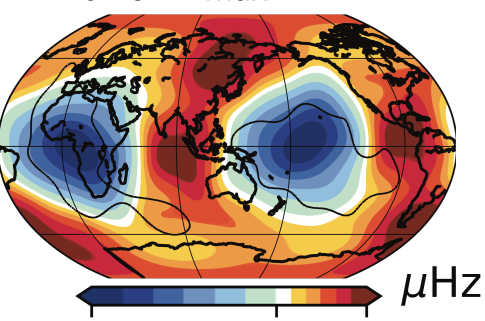

0.000 .13

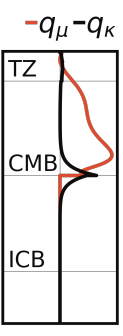

$-0.28$

${ }_{1} S_{9}, S_{\max }=10$

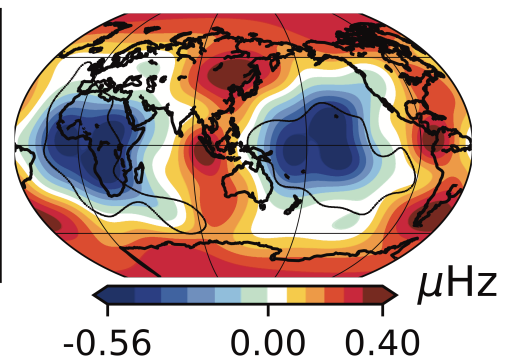

Figure 5.A.4: Elastic splitting function predictions of S20RTS (left), together with the anelastic splitting function predictions of S20RTS scaled using $R_{q}=-0.2$ in the upper mantle $(<660 \mathrm{~km})$ and $R_{q}=+0.2$ in the lower mantle (right). Splitting functions are plotted together with their respective sensitivity kernels for $v_{s}$ and $q_{\mu}$ (red lines), $v_{p}$ and $q_{\kappa}$ (black lines) and $\rho$ (grey line). Top: ${ }_{2} S_{12}$, dominated by $v_{s}$ sensitivity to the upper mantle. Middle: ${ }_{0} S_{6}$, dominated by $v_{s}$ sensitivity to the mid mantle. Bottom: ${ }_{1} S_{9}$, dominated by $v_{s}$ sensitivity to the lower mantle. 
S20RTS $c_{s t}$ predictions
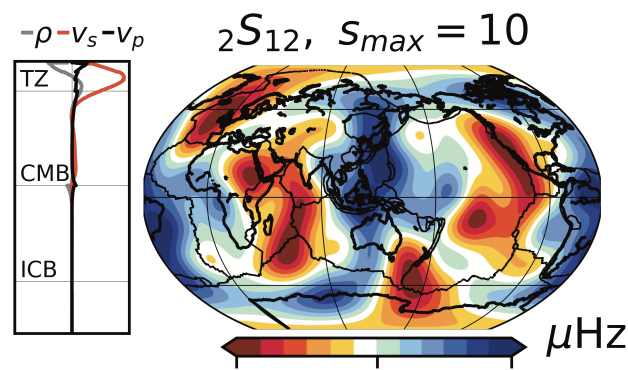

$\begin{array}{lll}-9.81 & 0.00 & 9.35\end{array}$

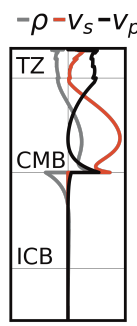

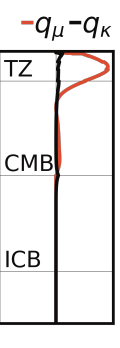

Upper mantle: $\delta q_{\mu}=-0.2 \delta v_{s} / v_{s}$

Lower mantle: $\delta q_{\mu}=+0.2 \delta v_{s} / v_{s}$

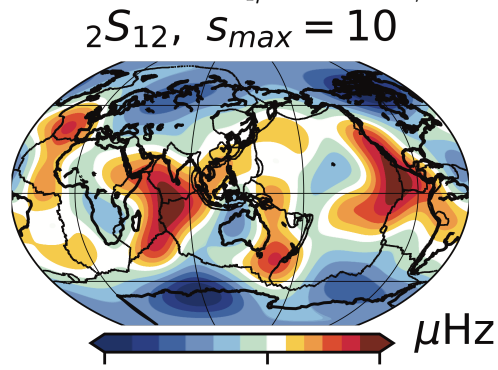

$\begin{array}{lll}-1.29 & 0.00 \quad 0.88\end{array}$

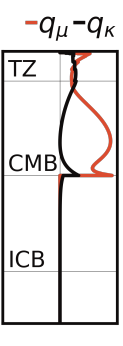

${ }_{0} S_{6}, S_{\max }=10$

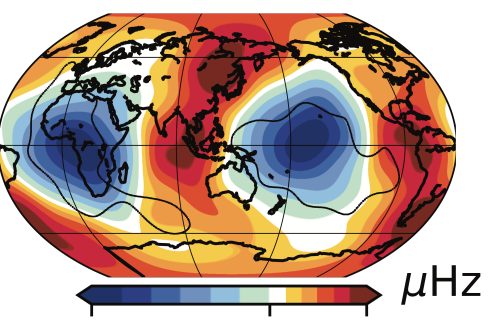

$\begin{array}{lll}-0.27 & 0.00 \quad 0.15\end{array}$

${ }_{1} S_{9}, S_{\max }=10$

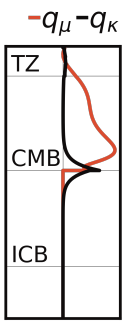

$\mu \mathrm{Hz}$

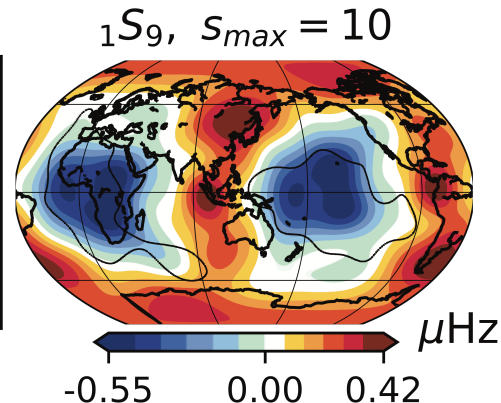

Figure 5.A.5: Elastic splitting function predictions of S20RTS (left), together with the anelastic splitting function predictions of S20RTS scaled using $R_{q}=-0.2$ in the upper mantle $(<450 \mathrm{~km})$ and $R_{q}=+0.2$ in the lower mantle (right). See the caption of Fig. 5.A.4 for explanation. 


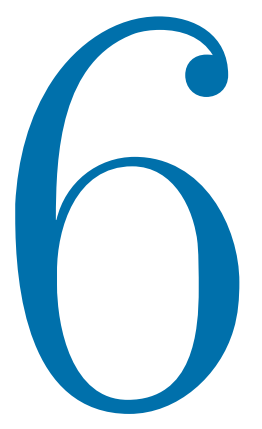

\section{Constraining 3D mantle attenuation}

Seismic tomographic models based solely on wave velocities have limited ability to distinguish between a temperature or compositional origin for Earth's 3D structure variations, such as the Large Low Shear Velocity Provinces (LLSVPs) beneath the lower mantle of Africa and the Pacific. Seismic attenuation or damping is able to provide additional information that may help to unravel the origin of the LLSVPs, which is fundamental to understand mantle convection evolution. For example, a thermal origin for the LLSVPs will point to them being short-lived anomalies, whereas a compositional origin will point to them being long-lived, forming mantle 'anchors' and influencing the pattern of mantle convection for a large part of Earth's history. Seismic attenuation is able to make that distinction, because it is directly sensitive to temperature variations. So far, global 3D attenuation models have only been available for the upper mantle, with only two regional body waves studies exploring the lower mantle (Lawrence and Wysession, 2006b; Hwang and Ritsema, 2011).

Here, we use normal mode data and first order perturbation theory to measure elastic splitting functions (dependent on velocity and density) and anelastic splitting functions (dependent on attenuation). The advantage of normal modes is that they are sensitive to the whole mantle and, just like surface waves (e.g. Karaoğlu and Romanowicz, 2018), they also allow us to include focussing and scattering due to the velocity structure without the need for further approximations, because we measure the elastic splitting function jointly with the anelastic splitting function. In our measurements of upper mantle sensitive modes, we find anti-correlation between the elastic and anelastic splitting functions, suggesting a thermal origin for low velocity spreading ridges, and agreeing with previous studies. On the other hand, for lower mantle sensitive modes, we find correlation. This suggests the averagely attenuating LLSVPs are warmer, large grain-size regions. While the 'ring around the Pacific' is a colder, small grain-size, strongly attenuating region, that could also contain potentially strongly attenuating post-perovskite. 


\subsection{Introduction}

Mantle convection is manifested at the Earth's surface through the observation of tectonic phenomena, like volcanic eruptions and earthquakes. However, to understand the driving forces behind mantle convection and if it is driven by only temperature or also compositional variation, we need to map the Earth's interior. Seismic tomography has been key in unravelling Earth's internal velocity structure. Unfortunately, seismic tomographic models based only on wave velocities are unable to distinguish between a temperature or compositional origin for Earth's 3D structure variations. The two most dominant features in tomographic shear wave velocity models are the Large Low Shear Velocity Provinces (LLSVPs) located in the lower mantle beneath Africa and the Pacific. The LLSVPs enfold roughly a quarter of the Core Mantle Boundary (CMB) region and vertically extend up-to 1,200 km above the CMB (Garnero et al., 2016). The main question regarding these large features is if they are only thermal, i.e. their lower velocity is due to a higher temperature, or if they also contain a different composition such as an increase in iron content. The origin of the LLSVPs is fundamental to understand mantle convection evolution, because a thermal anomaly will be short-lived (Zhang et al., 2010) and a significantly denser anomaly will be long-lived, forming mantle 'anchors' and influencing the pattern of mantle convection during long-periods of time (Torsvik et al., 2014).

In the past, studies aiming to determine the thermal versus the compositional heterogeneity of the mantle have mainly focused on studying density variations using whole Earth oscillations. The earliest studies found that LLSVPs have a larger density than the surrounding mantle (Ishii and Tromp, 1999; Trampert et al., 2004), which would imply that they have compositional heterogeneity, most likely due to enrichment in iron. However, studying density has proven challenging, with the latest studies finding either dense LLSVPs using tidal wave tomography (Lau et al., 2017), or neutrally buoyant or even light LLSVPs using CMB sensitive Stoneley ${ }^{1}$ modes (Koelemeijer et al., 2017). Some studies even report difficulties in robustly constraining density using inversion methods based on normal mode splitting functions (Masters et al., 2000c; Romanowicz, 2001; Akbarashrafi et al., 2017; Akbarashrafi, 2020). We need to add other constraints to solve this question and here we show that attenuation or damping, which is the intrinsic loss of energy as waves travel through the Earth, may provide the answer (e.g. Jackson and Faul, 2010a; Romanowicz and Mitchell, 2015).

\subsubsection{Attenuation from mineral physics}

Attenuation is sensitive to temperature, partial melt, water, grain-size and pressure, while it remains invariant to bulk composition (Faul and Jackson, 2005). Attenuation depends exponentially on temperature, which has been studied at length using both laboratory experiments and theoretical calculations (i.e. Faul and Jackson, 2015). The shear modulus, and consequently shear velocity, does not depend exponentially on temperature, instead it has a non-linear dependence which increases with increasing temperature (e.g. Faul and Jackson, 2005). Experiments using torsional forced

\footnotetext{
${ }^{1}$ Stoneley modes are a special class of modes confined to solid-liquid interfaces (Stoneley, 1924)
} 
oscillations on the upper mantle mineral olivine have shown that an increase in temperature lowers the shear modulus $\mu$, while it increases attenuation $q$ (Gribb and Cooper, 1998; Jackson and Faul, 2010a). This behaviour comes as a result of grain boundary sliding and depends strongly on the applied torsional frequency and grain size. As the grain size used in laboratory experiments is much smaller than the actual grain size in the mantle, extrapolations of the data are needed for its interpretation of seismic velocity and attenuation at mantle conditions (Faul and Jackson, 2005).

The relationship between attenuation and shear modulus becomes more complex when other variations are introduced to the olivine experiments. For example, the presence of a small melt fraction at the grain boundaries increases attenuation and lowers the shear modulus (Jackson et al., 2004; McCarthy and Takei, 2011). This means that regions with anomalously strong attenuation and slow velocities might be the result of partial melt in combination with high temperature. On the other hand, the presence of a small mass percentage of water seems to mainly increase attenuation as a result of a reduction in grain boundary viscosity, while it only slightly decreases the shear modulus as a result of dispersion (Karato, 2003; Shito et al., 2006; Aizawa et al., 2008). This means that regions showing strong attenuation together with a small velocity anomaly may be the result of the presence of water. In contrast, compositional variations are expected to have a strong effect on the shear velocity, but not on attenuation (Faul and Jackson, 2005). Thus, regions showing weak attenuation and a large shear velocity anomaly might be explained by compositional variations such as iron enrichment.

In Fig. 6.1, we reproduce a sketch based on Fig. 1 of Dalton et al. (2009), where the effects on olivine of the previously described parameters are illustrated in terms of attenuation and shear velocity, and as a result of lateral temperature variations at $150 \mathrm{~km}$ depth. Dalton et al. (2009) used the mineral physics model developed by Faul and Jackson (2005) in order to translate shear modulus, which was analysed in experiments, to seismic shear wave velocity. This model, which is derived from experiments and enables extrapolation to upper mantle conditions, is based on a modified Burgers rheological body and includes temperature and grain size dependence, as well as a distribution of anelastic relaxation times.

The relationship between attenuation and shear velocity for dry, melt-free olivine as a function of temperature is shown in Fig. 6.1a. We see strong attenuation and low velocities at high temperatures $\left(1400^{\circ} \mathrm{C}\right)$, and weak attenuation and high velocities at low temperatures $\left(400^{\circ} \mathrm{C}\right)$. The effect of additional variations on dry, melt-free olivine is shown in Fig. 6.1b. Increasing the magnesium content $(\mathrm{Mg})$, leads to an increase in shear velocity, but has minimal influence on attenuation (Lee, 2003). On the other hand, the presence of water, which is included in experiments as hydrogen $(\mathrm{H})$ related point defects in anhydrous minerals, increases attenuation, while it only influences shear modulus indirectly through dispersion (Karato and Jung, 1998; Karato, 2003). Finally, the presence of partial melt increases attenuation and decreases shear velocity. However, the nature of this effect depends on its mechanism. A melt-squirt mechanism seems to only lower the shear modulus, but has a negligible effect on attenuation (Hammond and Humphreys, 2000a; Hammond 


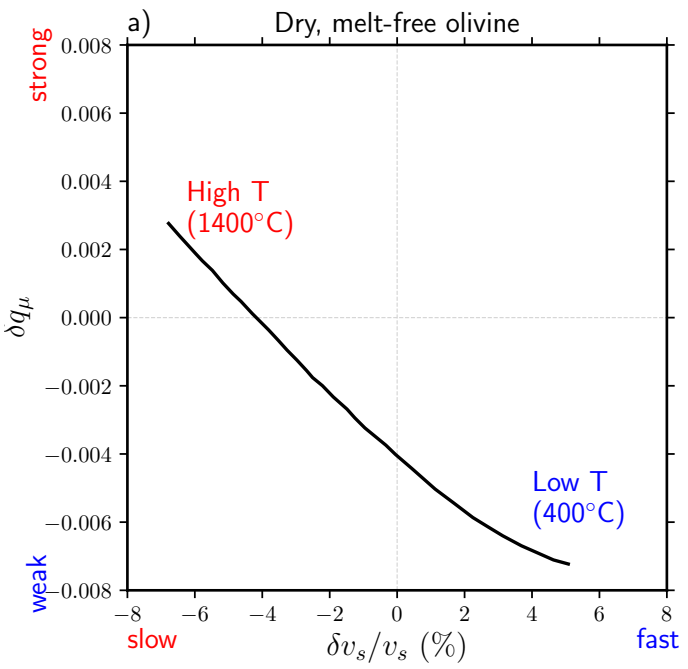

b) Olivine with additional parameter variations

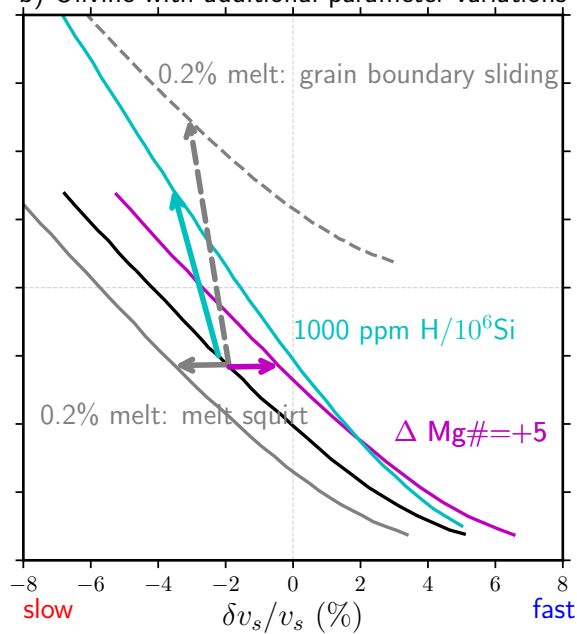

Figure 6.1: Sketch representing the relationship between attenuation $q_{\mu}$ and shear velocity $v_{s}$ for olivine, as a result of lateral temperature variations at $150 \mathrm{~km}$ depth, modified from Dalton et al. (2009). (a) The effect of temperature on dry, melt-free olivine $\left(400-1400^{\circ} \mathrm{C}\right)$ (solid black line); (b) showing additional effects, including a 5 unit increase in $\mathrm{Mg} \#$ i.e. $\Delta \mathrm{Mg} \#=+5$ or $\mathrm{Mg} \#=100 \times \frac{\mathrm{Mg}}{(\mathrm{Mg}+\mathrm{Fe})}=88-93$ (magenta line, Lee, 2003); a water content increase of 1000 ppm H/10 6 Si (cyan line, Karato, 2003; Yang et al., 2007; Harmon et al., 2009 ); the predicted effect of $0.2 \%$ melt assuming a melt-squirt mechanism (grey solid line, Hammond and Humphreys, 2000a; Hammond and Humphreys, 2000b); the predicted effect of $0.2 \%$ melt inferred from experimental grain boundary sliding data (grey dashed line, Jackson et al., 2004; Faul et al., 2004).

and Humphreys, 2000b), whereas grain boundary sliding enhanced by melt increases attenuation and lowers the shear modulus (Jackson et al., 2004; Faul et al., 2004).

Contrary to the upper mantle, there is only limited information on anelastic properties of lower mantle minerals. The torsional forced oscillation experiments required to study the temperature effect of shear modulus and attenuation are not possible at the high pressures required for lower mantle minerals. Thus, experiments have been performed on analogue minerals and minerals at lower pressures, such as titanate perovskites (Webb et al., 1999) and MgO (Webb and Jackson, 2003; Barnhoorn et al., 2016). The same effects as seen in olivine are also observed in both perovskite analogues and $\mathrm{MgO}$, with an increase in temperature decreasing the shear modulus and increasing attenuation. This means that if the LLSVPs have a thermal origin, then their low velocity will be accompanied by strong attenuation. If the LLSVPs are due to compositional heterogeneity instead, then no strong attenuation anomaly will be seen, because composition, and especially enrichment in iron with and without partial melt, is expected to have much less influence on attenuation than on shear wave velocity (e.g. Faul and Jackson, 2005). Even less is known on the attenuation properties of post-perovskite (Murakami et al., 2004; Oganov and Ono, 2004; Tsuchiya et al., 2004), which might be present in the deepest parts of the 
lower mantle. There is only one recent study using ab-initio molecular simulations which shows that post-perovskite is potentially very soft which means that it might be a strongly attenuating mineral (Goryaeva et al., 2016).

Attenuation also depends on grain-size, which in turn is pressure dependent (Faul and Jackson, 2005; Jackson and Faul, 2010b). Attenuation increases with smaller grain-sizes, while the shear modulus decreases (Faul and Jackson, 2005). However, small grain-sizes in combination with cold temperatures can lead to an increased attenuation and velocity, while large grain-sizes in combination with hot temperatures can lead to a decreased in attenuation and velocity (Dannberg et al., 2017). In particular, grain growth in post-perovskite is potentially much slower than perovskite, leading to smaller grains in regions containing post-perovskite (Yoshino and Yamazaki, 2007).

\subsubsection{Attenuation from seismology}

Few global 3D upper mantle attenuation models are currently available, and they have mainly been build using surface waves (e.g. Fig. 5.1). When making models of attenuation it is important to take into account focussing and scattering and correct for these effects, which are due to redistribution of energy and not due to intrinsic energy loss. Such corrections are difficult for body wave and surface wave studies and often involve approximations or extensive numerical calculations. As a consequence, global attenuation models have been difficult to make, and the different global attenuation models show less similarity than global shear wave velocity models.

The currently available upper mantle attenuation models show some similarity in the uppermost $200 \mathrm{~km}$ of the mantle, where strong attenuation has been found beneath spreading ridges and back-arcs, and weak attenuation is seen in shield regions (e.g. Romanowicz and Gung, 2002; Dalton et al., 2008; Zhu et al., 2013; Adenis et al., 2017; Karaoğlu and Romanowicz, 2018). Using Rayleigh waves, Romanowicz (1995) reported large global 3D attenuation variations in the upper mantle and linked them to structure found in global 3D shear velocity tomographic models. In particular, this first model confirmed that 3D attenuation in the transition zone is dominated by a well-resolved large degree-two structure (Romanowicz, 1990; Romanowicz, 1987; Roult et al., 1990), which was interpreted as evidence that 3D shear velocity variations in the upper mantle, which also show the same degree-two pattern, have a thermal origin. More recent attenuation models also agree on the dominant degreetwo structure in the upper mantle (e.g. Fig. 6.2). The agreement between the models is much smaller when including higher degrees (e.g. Fig. 5.1). But it is encouraging to see that models QRFSI12 (Dalton et al., 2008) and SEMUCB-UMQ (Karaoğlu and Romanowicz, 2018) do show higher correlation (above 0.6) in the upper $200 \mathrm{~km}$ of the mantle for higher degree structure, than other available $3 \mathrm{D} q_{\mu}$ models. At these depths, just like other earlier models, QRFSI12 and SEMUCB-UMQ show strong attenuation beneath spreading ridges and back-arcs, and weak attenuation in shield regions, which again suggests a thermal origin for low seismic velocity regions.

Constraints on lower mantle attenuation has been limited to three body wave studies (Romanowicz, 1998; Lawrence and Wysession, 2006b; Hwang and Ritsema, 2011). Lawrence and Wysession (2006b) identified a region in northeast Asia at the 

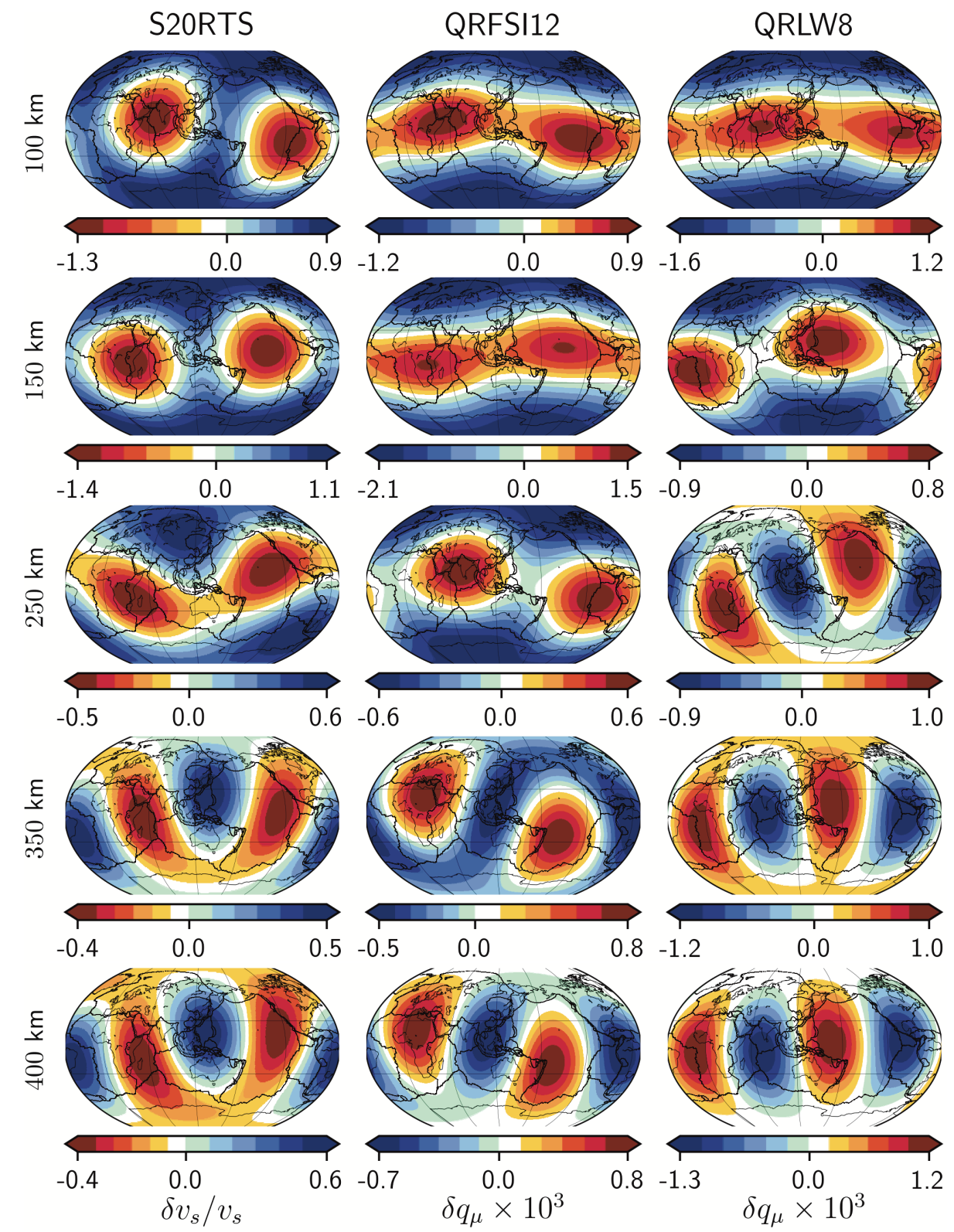

Figure 6.2: From left to right: 3D $v_{s}$ mantle model S20RTS, 3D $q_{\mu}$ upper mantle models QRFSI12 (Dalton et al., 2008) and QRLW8 (Gung and Romanowicz, 2004). All models are plotted for degree 2 in terms of $\delta q_{\mu} \times 10^{3}$ at five different depths: $100 \mathrm{~km}, 150 \mathrm{~km}$, $250 \mathrm{~km}, 350 \mathrm{~km}$ and $400 \mathrm{~km} . \delta q_{\mu} \times 10^{3}$ is presented as perturbation value and in percentage. Tectonic plate boundaries (Bird, 2003) are included for comparison. 
top of the lower mantle with anomalously strong attenuation and a slight decrease in velocity, and interpreted it as a subducting slab transporting water into the lower mantle. Hwang and Ritsema (2011) showed that the Pacific LLSVP was on average more strongly attenuating than its surrounding mantle for depths up-to $2470 \mathrm{~km}$. Romanowicz (1998) reported a stable degree-two pattern in the lowermost mantle, which showed strongly attenuating LLSVPs in a preliminary wave-form inversion for horizontally polarised shear-waves. Although the 3D attenuation model of Gung and Romanowicz (2004) did not extend into the lower mantle, they linked the strong attenuation in their models in the transition zone under Africa and the Pacific to hotspot locations, and used this as evidence for coherent upwellings rising from the LLSVPs at the base of the lowermost mantle all the way up-to the transition zone (Romanowicz and Gung, 2002).

Here, we use normal modes, or whole Earth oscillations to measure 3D variations in attenuation for both the upper and lower mantle using normal mode splitting functions. The advantage of using normal modes is that it allows us to include focussing and scattering, without the need for approximations. This is achieved because the $3 \mathrm{D}$ variations in velocity and attenuation are measured jointly. In addition, due to the large wavelengths of the standing waves, scattering off smallscale structure is less of a problem. We measure the attenuation of the normal modes using splitting functions, which are depth-averaged models of how one particular mode 'sees' the Earth. Most often only the elastic coefficients $c_{s t}$ of the splitting functions are measured, which provides information on 3D variations in velocity and density (e.g. Deuss et al., 2013). Here, we extend this to also include the anelastic coefficients $d_{s t}$ of the splitting function, which depends on 3D attenuation variations (e.g. Mäkinen and Deuss, 2013). By measuring the elastic and anelastic coefficients simultaneously, we include focussing effects (and implicitly correct for them) in the attenuation measurements.

\subsection{Method and Data}

We now focus on measuring elastic and anelastic splitting functions using observed normal mode spectra for real earthquakes, building on the experience gained in the tests on synthetic data performed in Chapter 5. The inversion scheme used is the iterated least-squares inversion (Tarantola and Valette, 1982), following the same methodology and misfit calculation as Deuss et al. (2013) and Mäkinen and Deuss (2013), as explained in Chapter 2. We use the earthquake catalogue published by Deuss et al. (2013) and Koelemeijer et al. (2013), and extended it to add 14 additional large earthquakes that occurred between 2012 and 2018 (Table 2.1). Horizontal data was also added to the catalogue (Schneider and Deuss, 2020). The extended catalogue now consists of 107 earthquakes with magnitude $M_{w}>7.4$ which occurred in the period from 1975 to 2018, providing us with an average increase of $24 \%$ in number of spectra per mode, compared to Deuss et al. (2013). Our observations will be compared to the ones obtained by Deuss et al. (2013) later in this chapter.

Based on our results in Chapter 5, where we inverted for synthetic anelastic splitting functions, we expect that the anelastic splitting function coefficients $d_{s t}$ measured from real data will be on average around 10 times smaller than the elastic 
splitting function coefficients $c_{s t}$. Because of this reason, we first measure the larger $c_{s t}$ coefficients on their own. In the second step of the measurement process, we use these $c_{s t}$ measurements as our starting model, and then measure $c_{s t}$ and $d_{s t}$ coefficients simultaneously, while starting all $d_{s t}$ measurements from PREM (i.e. having zero values, Dziewonski and Anderson, 1981). This approach was tested using synthetic data (see Section 5.3), where we confirmed that, even in the synthetic case, the $d_{s t}$ coefficients can only be recovered when the $c_{s t}$ coefficients were measured first and later used as a starting model in a joint $c_{s t}$ and $d_{s t}$ inversion.

Furthermore, to avoid aliasing, all $c_{s t}$ measurements for a given mode ${ }_{n} S_{l}$ were done up-to their highest possible structural degree $\left(s_{\max }=2 l\right)$. The $d_{s t}$ coefficients of modes with $2 l \leq 12$ were also measured up-to their highest possible structural degree $\left(s_{\max }=2 l\right)$. However, modes with $2 l>12$ were measured to a lower structural degree $\left(s_{\max }=12\right)$. For these modes not enough data is available to measure both $c_{s t}$ and $d_{s t}$ coefficients up-to the highest structural degree. For example, the $c_{s t}$ coefficients of mode ${ }_{2} S_{12}$ were measured up-to $s_{\max }=24$, while its $d_{s t}$ coefficients were measured up-to $s_{\max }=12$.

Mode pairs ${ }_{1} S_{6-2} S_{5}$ and ${ }_{1} S_{5-2} S_{4}$ were measured in group-coupling. For these modes, the self-coupling splitting functions are measured using the same procedure described above. The elastic cross-coupling was measured up to its highest possible structural degree, while the anelastic cross-coupling was measured up to $s_{\max }=5$.

\subsubsection{Damping}

As explained in the previous section, we first measure the larger $c_{s t}$ coefficients on their own and apply the same damping to all coefficients. In the second step, we use the $c_{s t}$ measurements as our starting model, and then measure $c_{s t}$ and $d_{s t}$ coefficients simultaneously, and again apply the same damping to all coefficients. We select the optimal damping value by evaluating the misfit, the squared model size and the effective number of independent model parameters as a function of iteration and damping (see Figs. 6.A.1-6.A.2).

\subsubsection{Influence of the starting model}

Before analysing our observations, we first investigate if our 3D anelastic measurements are not the result of a local minimum, and if there is enough information in the data to move away from the starting model and not stay too close to it in the inversion. In order to do this, we test the effect of different starting models on our anelastic splitting function measurements. We find that using an anelastic starting model either correlated $\left(R_{q}=0.2\right)$ or anti-correlated $\left(R_{q}=-0.2\right)$ to the $3 \mathrm{D}$ elastic model S20RTS or even a starting model that contains zeroes (PREM), does not influence our final $d_{s t}$ observations. We always arrive at the same $d_{s t}$ coefficients within the measurement's uncertainties (Fig. 6.3). This means that we do not need to include any a priori assumptions about the relationship between elastic and anelastic structure in order to measure 3D anelasticity, and therefore we always start our $d_{s t}$ measurement from PREM (i.e. zero values). For the modes where this was not possible, and the measured $d_{s t}$ coefficients were found to be dependent on the starting model, we decided not to report the $d_{s t}$ observations (Table 6.1). 
a) ${ }_{2} S_{12}$

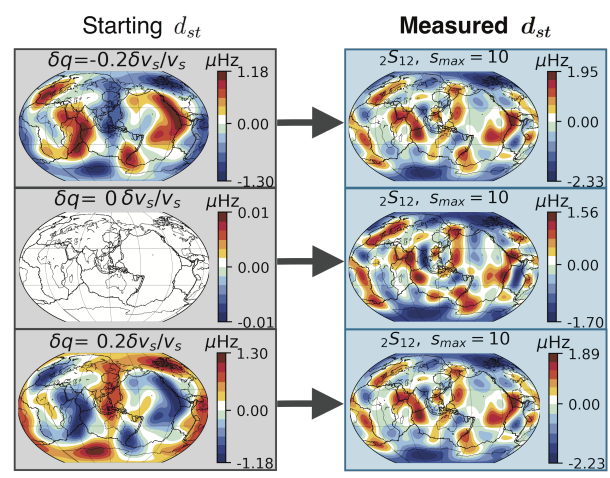

b) ${ }_{1} S_{10}$

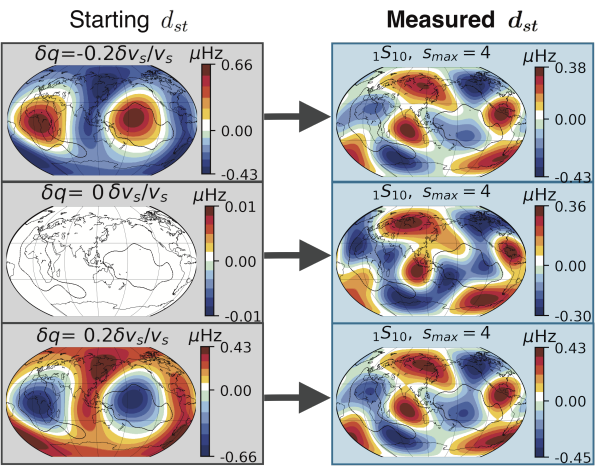

Figure 6.3: Anelastic splitting function $d_{s t}$ measurements for real data using different starting models in the inversion. Splitting functions maps are $d_{s t}$ are shown for (a) upper mantle sensitive mode ${ }_{2} S_{12}$ (also shown are the tectonic plate boundaries (Bird, 2003)), and (b) lower mantle sensitive mode ${ }_{1} S_{10}$ (with the $-0.1 \% v_{s}$ outline of the tomographic model SP12RTS at $2850 \mathrm{~km}$ (Koelemeijer et al., 2016), which contours the LLSVPs). The grey panels show the starting model $d_{s t}$ used in the inversion, with the title of each sub-figure outlining the characteristics of the starting model $d_{s t}$. These starting model $d_{s t}$ were obtained by scaling the elastic model S20RTS (Ritsema et al., 1999) using $\delta q=R \delta v_{s} / v_{s}$. The blue panels show the measured $d_{s t}$ together with the $s_{\max }$ plotted for each measured $d_{s t}$.

\subsubsection{Normal mode selection}

In order to determine the mantle sensitive modes for which we measure anelastic splitting functions, we applied the following selection criteria to the normal mode catalogue of elastic splitting functions published by Deuss et al. (2013):

(i) modes measured using the self-coupling approach, so excluding fundamental modes with strong Coriolis coupling to toroidal modes (e.g. $\left.{ }_{0} S_{9{ }^{-} 0} T_{10}\right)$;

(ii) modes with $l \leq 13$, in order to be able to parameterise all $c_{s t}$ coefficients to their $s_{\max }$, avoiding aliasing between $c_{s t}$ and $d_{s t}$ coefficients and keeping the number of inverted coefficients below 500;

(iii) modes for which the number of spectral segments is an order of magnitude larger than the number of inverted coefficients.

When we measure elastic and anelastic splitting functions simultaneously, we double the amount of unknown model parameters in the inversion (i.e. $c_{s t}$ and $d_{s t}$ coefficients), which becomes more significant for modes ${ }_{n} S_{l}$ with larger angular order $l$. Furthermore, the frequency separation between modes with larger $l$ decreases, which makes the task to isolate modes in self-coupling more difficult and higher degree cross-coupling between modes becomes more important. In this thesis, the only mode pairs measured in group-coupling are ${ }_{1} S_{5-2} S_{4}$ and ${ }_{1} S_{6-2} S_{5}$. They have 
Table 6.1: Modes for which elastic and anelastic splitting functions are measured in this thesis. Spheroidal modes were selected from the elastic splitting functions observations of Deuss et al. (2013), and toroidal modes from Schneider and Deuss (2020). Modes marked with $\dagger$ depend on the starting model, modes marked with $\ddagger$ are beyond $4 \mathrm{mHz}$ and cannot be measured starting from PREM, and modes marked with $\nmid$ have too large $d_{s t}$ uncertainties or change their $c_{s t}$ values significantly when measured together with $d_{s t}$.

\begin{tabular}{lll}
\hline & Fundamentals & ${ }_{0} S_{2},{ }_{0} S_{5},{ }_{0} S_{6},{ }_{0} S_{7}$ \\
& $1^{\text {st }}$ overtones & ${ }_{1} S_{4},{ }_{1} S_{5},{ }_{1} S_{6},{ }_{1} S_{7},{ }_{1} S_{8},{ }_{1} S_{9},{ }_{1} S_{10}$ \\
& $2^{\text {nd }}$ overtones & ${ }_{2} S_{4},{ }_{2} S_{5},{ }_{2} S_{6},{ }_{2} S_{12},{ }_{2} S_{13}$ \\
& $3^{\text {rd }}$ overtones & ${ }_{3} S_{6}^{\dagger},{ }_{3} S_{9}$ \\
\cline { 2 - 3 } Spheroidal modes & $5^{\text {th }}$ overtones & ${ }_{5} S_{3}^{\dagger},{ }_{5} S_{7}^{\dagger},{ }_{5} S_{8}^{\dagger},{ }_{5} S_{12}^{\ddagger}, \dagger$ \\
& $6^{\text {th }}$ overtones & ${ }_{6} S_{10}^{\ddagger}$ \\
& $7^{\text {th }}$ overtones & ${ }_{7} S_{7}^{\ddagger}$ \\
& $8^{\text {th }}$ overtones & ${ }_{8} S_{6}^{\ddagger},{ }_{8} S_{7}^{\ddagger}$ \\
& $9^{\text {th }}$ overtones & ${ }_{9} S_{11}^{\ddagger}$ \\
& $11^{\text {th }}$ overtones & ${ }_{11} S_{9}^{\ddagger}$ \\
& $12^{\text {th }}$ overtones & ${ }_{12} S_{6}^{\ddagger},{ }_{12} S_{7}^{\ddagger},{ }_{12} S_{13}^{\ddagger}$ \\
\hline Toroidal modes & $1^{\text {st }}$ overtones & ${ }_{1} T_{2}^{\dagger},{ }_{1} T_{3}^{\dagger},{ }_{1} T_{6}^{\dagger}$ \\
\hline
\end{tabular}

been selected given their low angular order $l$ and large number of data available, which allows these mode pairs to be modelled by fewer splitting function coefficients than other higher $l$ mode pairs. In addition to spheroidal modes, we also tried to measure anelastic splitting functions of some of the isolated toroidal modes reported in Schneider and Deuss (2020).

In Table 6.1, we list all modes from Deuss et al. (2013) and Schneider and Deuss (2020) that satisfied the initial selection criteria. After making the measurements for all modes listed in Table 6.1, we found out that only the anelastic splitting functions of spheroidal modes with $n=0,1,2,3$ are well constrained (Table 6.2). We had to discard all anelastic measurements of modes above $4 \mathrm{mHz}$, because these modes all required a starting anelastic model different from zero values for $d_{s t}$. Additionally, we also discarded all anelastic splitting functions measurements that depend on the starting model, or that had unstable inversion results for either the elastic or anelastic splitting function coefficients. Ultimately, this meant that we were only able to measure robust 3D anelastic splitting functions for normal modes below $3 \mathrm{mHz}$. Table 6.1 highlights per mode the reasons for which a mode measurement has been discarded. 


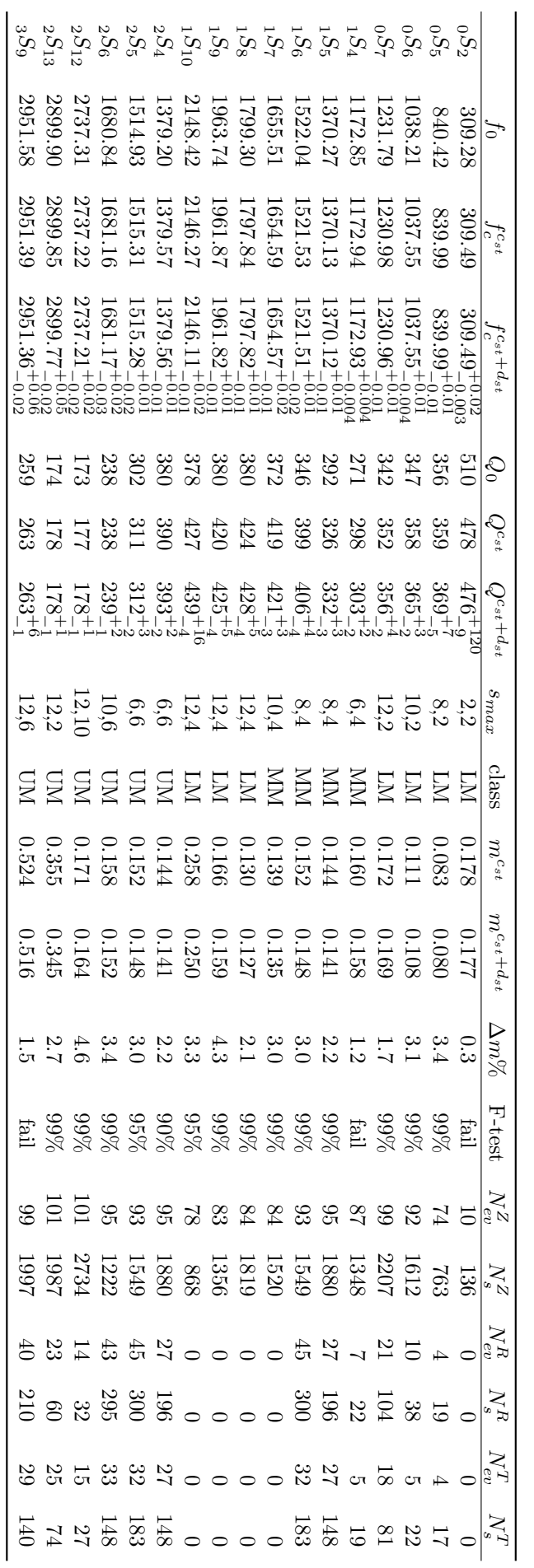

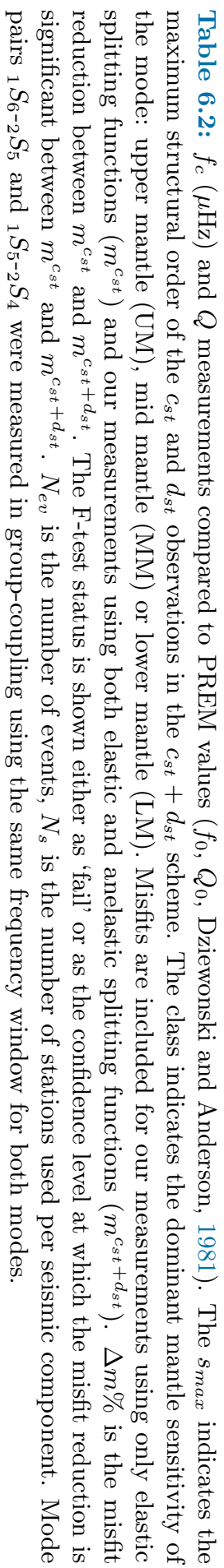


(1) पि

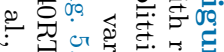

N

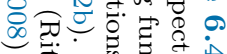

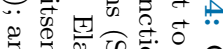

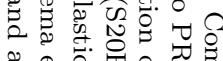

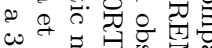

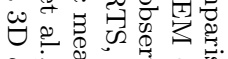

늘

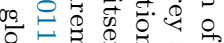

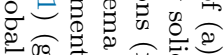

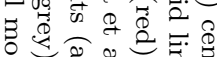

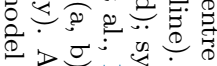

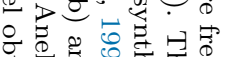

过.

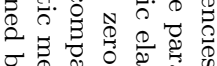

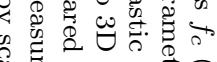

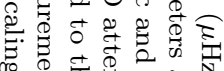

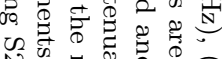

กิ่ง

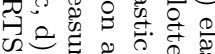

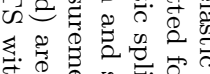

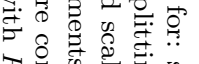

ऽ。
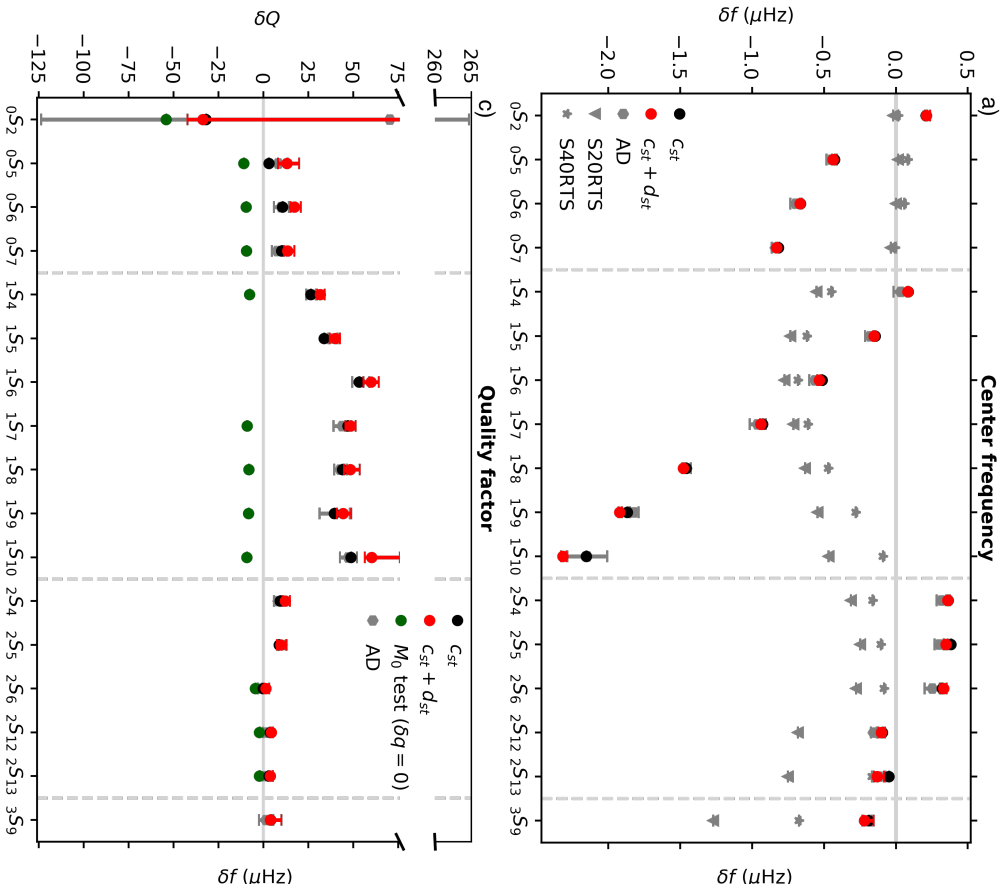

||

$1 \stackrel{0}{\circ} 0$

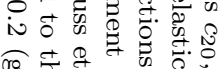

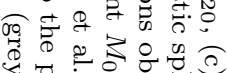

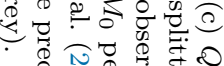

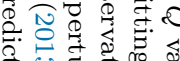

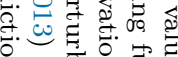

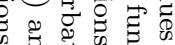

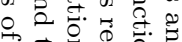

इ 㕝它

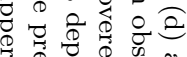

उ

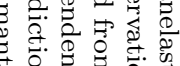

के

క

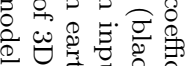

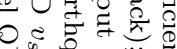

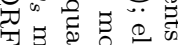

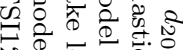

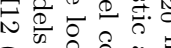

ठै

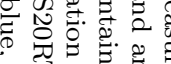

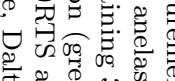

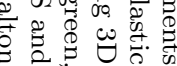

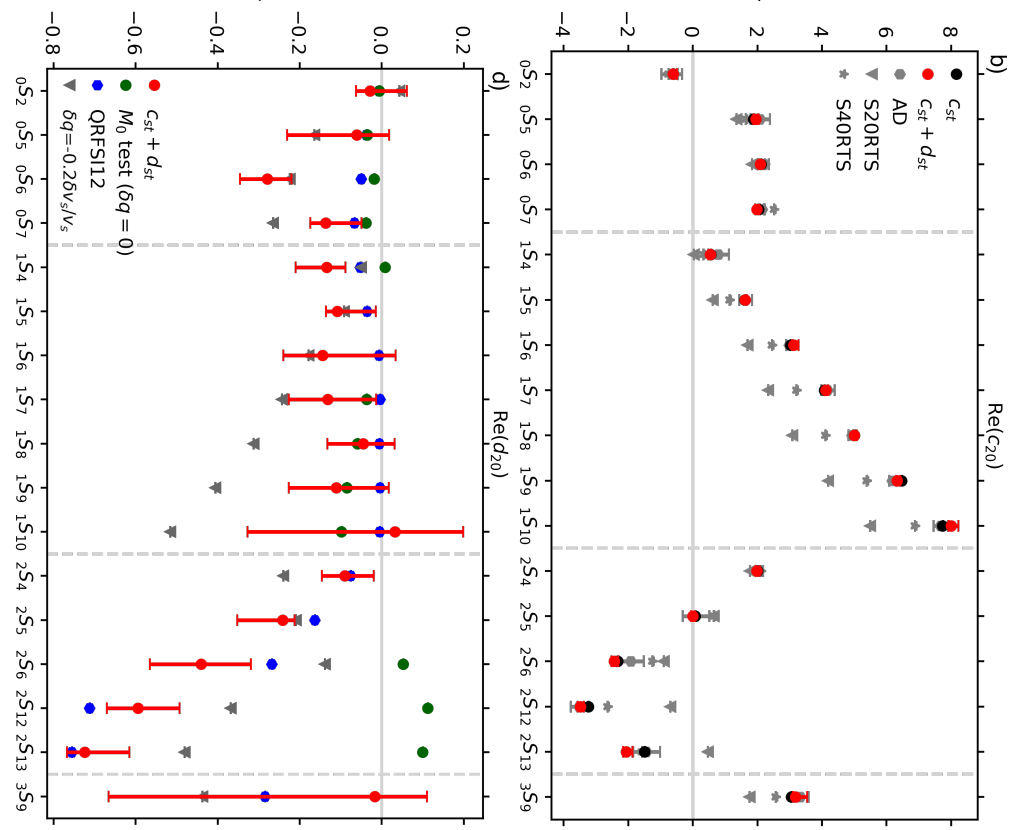




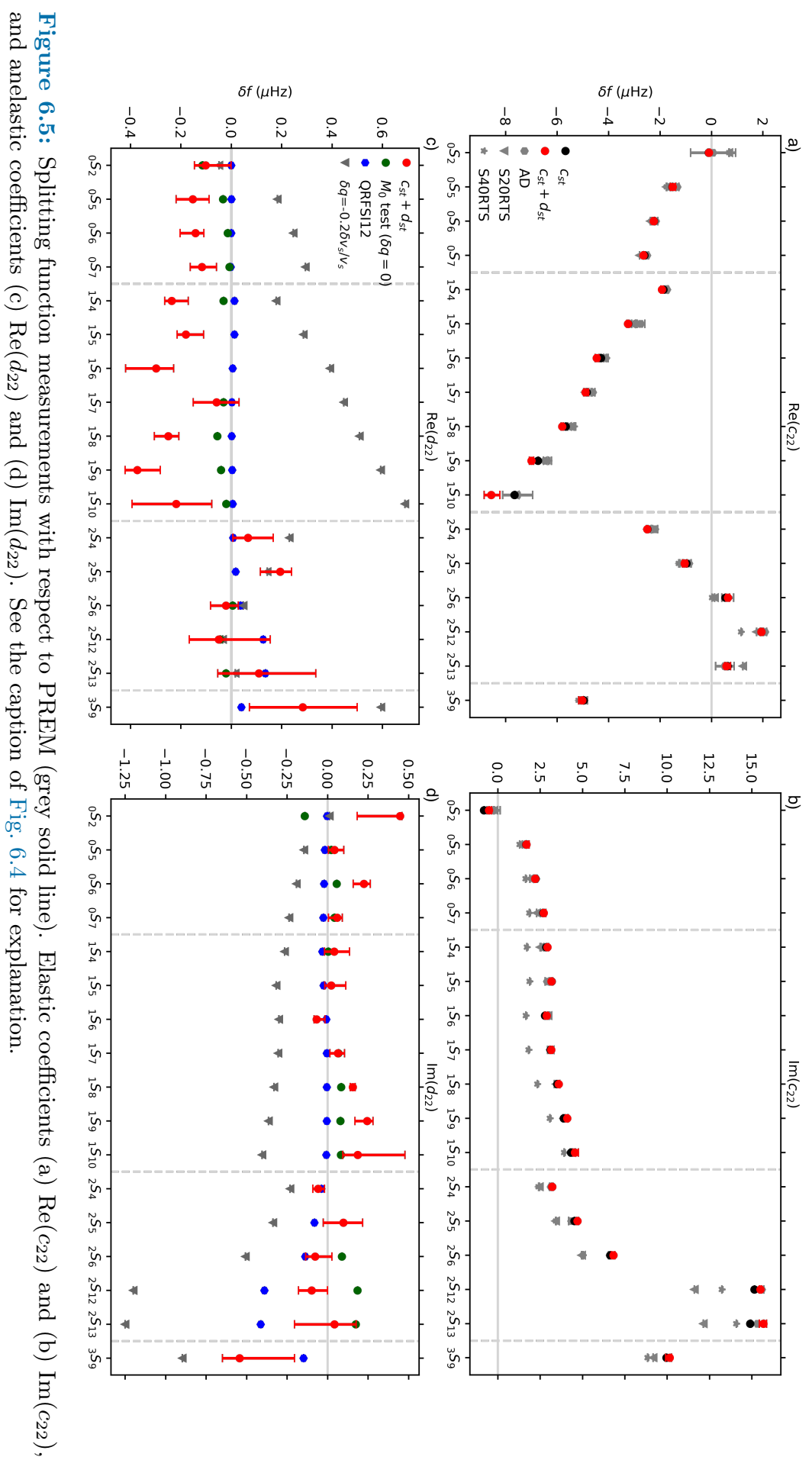




\subsection{Results}

We measured anelastic splitting function coefficients for 13 spheroidal modes in self-coupling, and two spheroidal mode pairs in group-coupling (Figs. 6.4, 6.5, 6.B.4). When comparing the misfit of our 3D elastic measurement inversion to the misfit of our combined 3D elastic and anelastic inversion (Table 6.2), we find a statistically significant but relatively small misfit reduction $(\sim 3 \%)$ when the anelastic splitting function is included compared to only including the elastic spitting function (Fig. 6.6). This is especially evident when comparing our results to the larger misfit reductions $(\sim 13 \%)$ found in similar measurements for inner core sensitive modes (Mäkinen and Deuss, 2013). However, from our forward modelling in Section 5.2.3, we know that including $3 \mathrm{D}$ anelastic mantle variations of comparable size to current $3 \mathrm{D} q_{\mu}$ upper mantle models (e.g. Dalton et al., 2008) in the calculation of synthetic normal
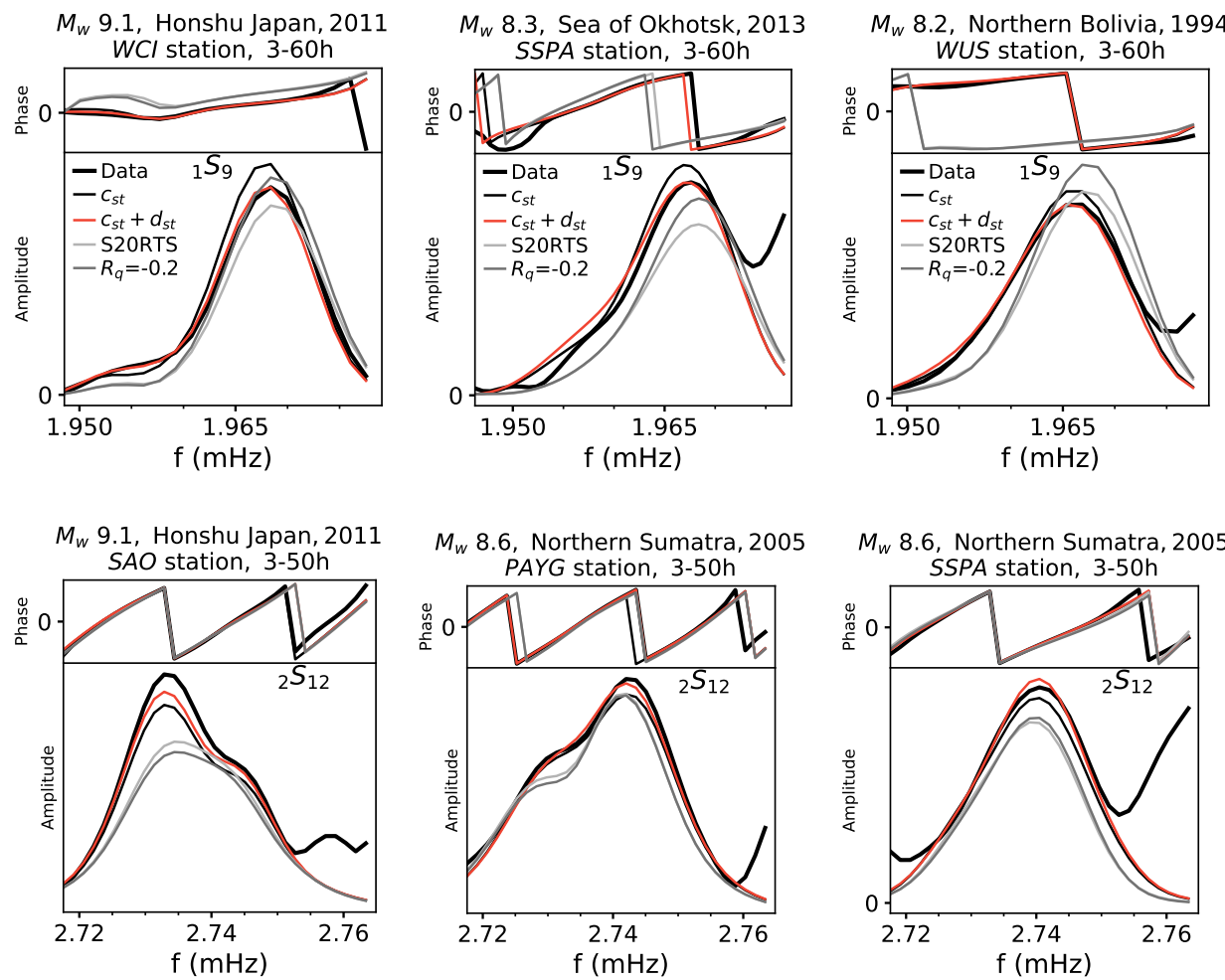

Figure 6.6: Observed data (thick black line) and synthetics for modes ${ }_{1} S_{9}$ (top) and ${ }_{2} S_{12}$ (bottom). Synthetics are calculated for: our observations including only elastic splitting functions (black thin line); our observations including elastic and anelastic splitting functions (red line); S20RTS (light grey line, Ritsema et al., 1999); and S20RTS together with a 3D $q_{\mu}$ global model obtained by scaling S20RTS with $R_{q}=-0.2$ (dark grey line). 
mode spectra, results in a difference in misfit to the spectral data of between $1.5 \%$ and $4 \%$, when compared to synthetic spectra containing only $3 \mathrm{D}$ elastic structure. Furthermore, in tests conducted in Section 5.3, we find that our measurements are well constrained and argue that the reason for our low misfit reduction, when compared to similar measurements of inner core sensitive modes (see Mäkinen and Deuss, 2013), is that 3D anelasticity has a much stronger effect in inner core sensitive modes than in the mantle sensitive modes.

It is also important to note that, 3D elastic anomalies, which perturb the frequency of a normal mode or the velocity of a wave as they travel through it, can be cancelled when travelling through fast and slow velocity anomalies. Anelastic anomalies, on the other hand, are cumulative and cannot be cancelled. This means that a wave which loses energy as it passes through an anelastic anomaly, will not be able to gain this energy back later. This implies that the amplitude of a normal mode will not only get smaller as a wave travels deeper into the mantle, but that this cumulative effect might make lower mantle sensitive modes also show upper mantle anelastic structure, in addition to lower mantle structure. More simply put, the lower mantle sensitive modes are also sensitive to upper mantle structure, both elastic and anelastic.

For all the spheroidal modes we measured here, the elastic splitting functions are dominated by $v_{s}$ mantle sensitivity, and the anelastic functions are dominated by $q_{\mu}$ mantle sensitivity. To better interpret our anelastic splitting function observations, we classify our measured modes (Table 6.2) by their $q_{\mu}$ sensitivity to either the upper, mid or lower mantle (Fig. 6.7), in the same manner as in Chapter 5. Modes showing $q_{\mu}$ sensitivity to both the upper and lower mantle, are classified based on their agreement with other modes sensitive solely to the upper or lower mantle (i.e. ${ }_{3} S_{9}$ ). As a result, the upper mantle sensitive $q_{\mu}$ modes are ${ }_{2} S_{4},{ }_{2} S_{5},{ }_{2} S_{6},{ }_{2} S_{12},{ }_{2} S_{13},{ }_{3} S_{9}$; the mid mantle sensitive $q_{\mu}$ modes are ${ }_{1} S_{4},{ }_{1} S_{5},{ }_{1} S_{6},{ }_{1} S_{7}$; and the lower mantle sensitive $q_{\mu}$ modes are ${ }_{0} S_{2},{ }_{0} S_{5},{ }_{0} S_{6},{ }_{0} S_{7},{ }_{1} S_{8},{ }_{1} S_{9},{ }_{1} S_{10}$. For the interpretations later in this chapter, it is important to note that all the $q_{\mu}$ sensitivity kernels are

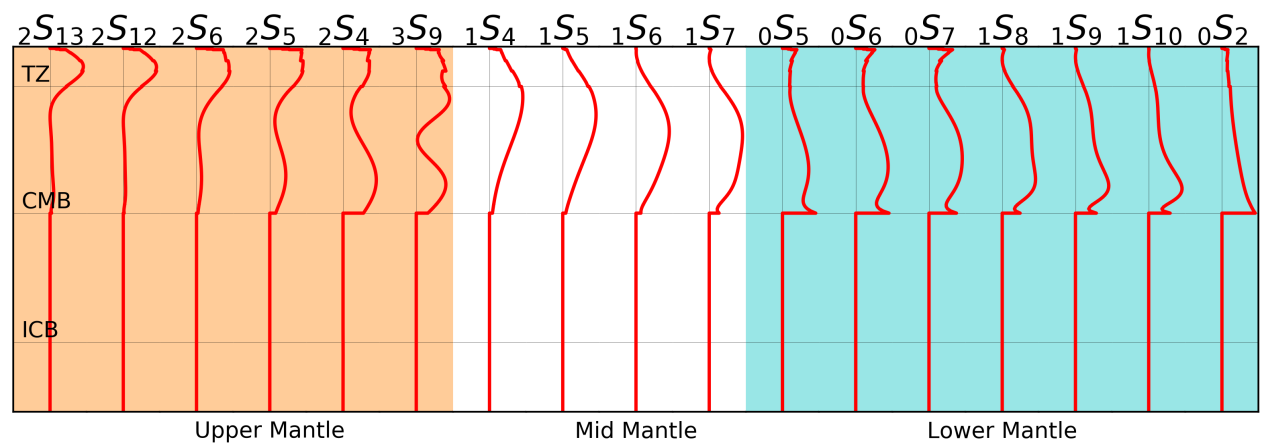

Figure 6.7: Sensitivity kernels as a function of depth for shear attenuation $q_{\mu}\left(\mu_{0} K_{\mu}\right)$, calculated for PREM. Modes are arranged and classified according to where they are most sensitive. The transition Zone (TZ), Core Mantle Boundary (CMB) and Inner Core Boundary (ICB) are marked in figure. All kernels are plotted for degree-zero. 
positive for all mantle depths, which means that positive frequency anomalies in the $d_{s t}$ splitting function maps correspond to locally stronger attenuation and negative anomalies to weaker attenuation.

No global whole mantle 3D attenuation model is currently available, so we will compare our anelastic splitting function measurements to predictions for both upper mantle 3D $q_{\mu}$ model QRFSI12 (Dalton et al., 2008), and our whole mantle synthetic 3D $q_{\mu}$ model, which is obtained by applying the scaling $\delta q_{\mu}=R_{q} \delta v_{s} / v_{s}$ to the $v_{s}$ model S20RTS (Ritsema et al., 1999). We use $R_{q}=-0.2$, which was estimated based on a comparison between the upper mantle 3D $q_{\mu}$ variations of model QRFSI12 and the 3D $v_{s}$ variations of model S20RTS in Section 5.2. This $R_{q}$ value was later extrapolated to the whole mantle. Thus, in our synthetic model, the $3 \mathrm{D} q_{\mu}$ variations are anti-correlated to the 3D $v_{s}$ variations of S20RTS both in the upper and lower mantle (Section 5.2).

\subsubsection{Fundamentals and $1^{\text {st }}$ overtone modes}

We find that our fundamental and first overtone splitting functions measurements show the smallest anelastic signal, when comparing all anelastic splitting functions measured in this chapter. This is to be expected, because our synthetic forward modelling using $R_{q}=-0.2$ also resulted in the smallest predicted anelastic signal for these modes (see Section 5.2.3). Our synthetic forward modelling also indicated that the $d_{s t}$ amplitude increases for fundamental and first overtone modes as they become more sensitive to the lower mantle, e.g. ${ }_{0} S_{2},{ }_{0} S_{5},{ }_{0} S_{6},{ }_{0} S_{7},{ }_{1} S_{8},{ }_{1} S_{9},{ }_{1} S_{10}$, and we will show below that the same effect is seen in our anelastic splitting function observations for real earthquake data. It is also important to point out that, as seen in Section 5.3.2, elastic effects might manifest themselves within our anelastic observations in a branch consistent manner. However, if this was the case, we would expect to obtain comparable anelastic signals along a mode branch with increasing angular order and depth sensitivity, which is not what we see (Figs. 6.4, 6.5, 6.B.4).

We measure all our elastic splitting functions up-to $s_{\max }=2 l$ and our anelastic splitting functions up-to $s_{\max } \leq 12$ as explained in Section 6.2. We found that our anelastic splitting function measurements are only robust for much lower $s_{\max }$ values, and we only want to interpret the robust part of the measurements. We find that the anelastic splitting functions of fundamental modes for $s_{\max } \geq 4$, as well as of first overtone modes for $s_{\max } \geq 6$ are not well constrained. For this reason, we use $s_{\max }=2$ as the maximum structural order for the anelastic splitting functions of fundamental modes, and $s_{\max }=4$ for the first overtone modes (Table 6.2), which we also validated by analysing the branch consistency of the measured $d_{s t}$. Therefore, fundamental mode splitting functions measurements are only shown for $s_{\max }=2$ in Fig. 6.8; the measurements of the first overtone modes are only shown for $s_{\max }=2,4$ in Figs. 6.9-6.10. Nevertheless, for completeness, we do include the observed splitting functions of the fundamental modes for $s_{\max }=4,6$ and of the first overtones for $s_{\max }=6$ in Section 6.B (see Figs. 6.B.1-6.B.3).

All our anelastic splitting function maps for $n=0,1$ are branch consistent and show positive and negative frequency anomalies, i.e. strong and weak attenuation, in the same places. When considering only degree-two structure, all our measurements 
Our $c_{s t}$ measurements

Our $d_{s t}$ measurements

S20RTS $d_{s t}$ predictions QRFSI12 $d_{\text {st }}$ predictions

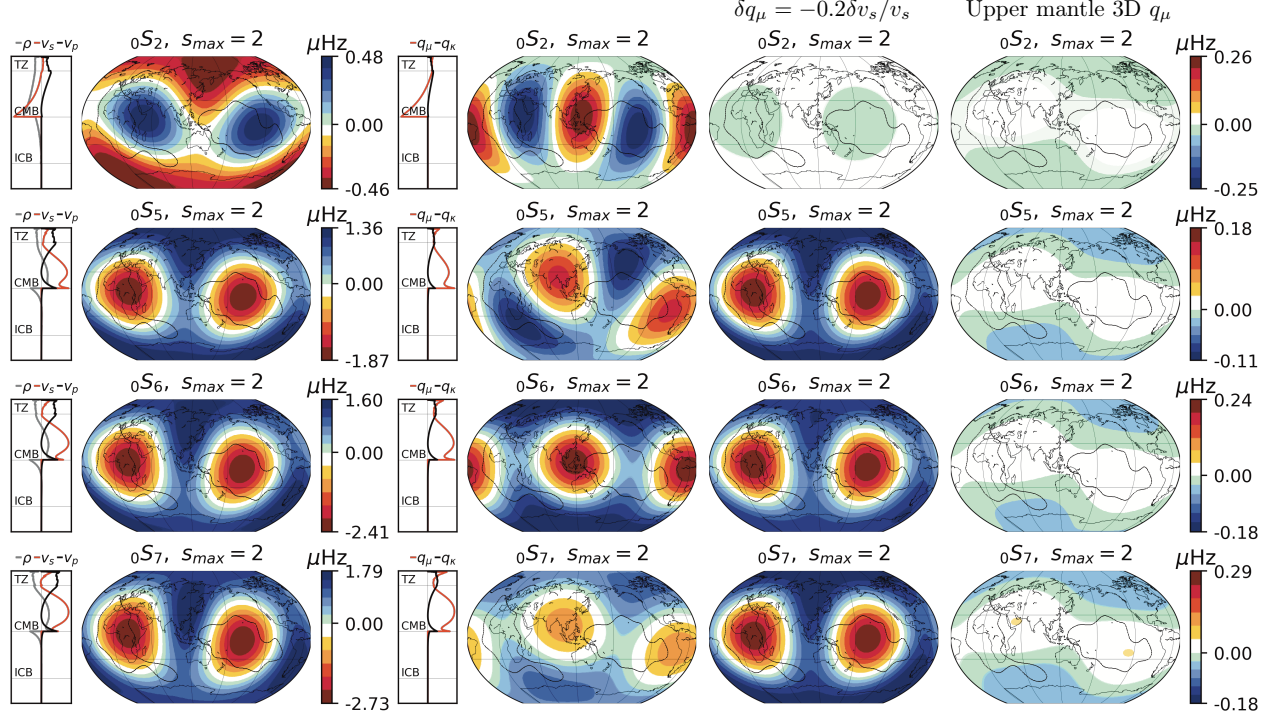

Figure 6.8: Splitting function $c_{s t}\left(1^{\text {st }}\right.$ column $)$ and $d_{s t}\left(2^{\text {nd }}\right.$ column $)$ measurements of fundamental modes ${ }_{0} S_{l}$, plotted together with their respective sensitivity kernels for $v_{s}$ and $q_{\mu}$ (red lines), $v_{p}$ and $q_{\kappa}$ (black lines) and $\rho$ (grey line). All kernels are calculated using PREM for degree-zero, with the exception of mode ${ }_{0} S_{2}$, which is shown for degree 2 . Measurements are compared to anelastic splitting function predictions for $s_{\max }=2$ for our 3D synthetic anelastic model ( $3^{\text {rd }}$ column) and 3D anelastic upper mantle model QRFSI12 (up-to $400 \mathrm{~km})\left(4^{\text {th }}\right.$ column, Dalton et al., 2008). The $-0.1 \% v_{s}$ outline of the tomographic model SP12RTS at $2850 \mathrm{~km}$ (Koelemeijer et al., 2016), which contours the LLSVPs, is included for comparison.

of fundamental (except ${ }_{0} S_{2}$ ) and first overtone modes show anelastic structure which appears to be correlated to the elastic structure (Figs. 6.8-6.9). When we also include degree-four structure, the correlation becomes less clear and more features become visible in the anelastic splitting function maps than in the elastic splitting function maps (Figs. 6.10, 6.B.1). With the exception of mode ${ }_{0} S_{2}$, all other fundamental and first overtone modes measurements for $s_{\max }=2$ show the most strongly attenuating structure not in the LLSVP regions, but in the 'ring around the Pacific' instead, with maxima below Indonesia and central America (Figs. 6.8-6.10). When comparing to the elastic structure, these two strongly attenuating regions appear to be shifted westwards of Indonesia and Central America for the first overtones which are sensitive to the mid-mantle $\left({ }_{1} S_{4},{ }_{1} S_{5},{ }_{1} S_{6}\right)$. These two regions then move eastwards towards Indonesia and Central America for the first overtones which are sensitive to the lower mantle $\left({ }_{1} S_{8},{ }_{1} S_{9},{ }_{1} S_{10}\right)$ (Fig. 6.9). However, this systematic pattern shift becomes less clear for $s_{\max }=4$ structure (Fig. 6.10).

When we compare our ${ }_{0} S_{l}$ and ${ }_{1} S_{l}$ measurements of degree-two and four to the predictions of the 3D $q_{\mu}$ upper mantle model QRFSI12 (Dalton et al., 2008), we 
Our $c_{s t}$ measurements

Our $d_{s t}$ measurements

S20RTS $d_{s t}$ predictions QRFSI12 $d_{s t}$ predictions

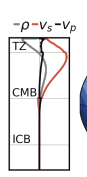

${ }_{1} S_{4}, S_{\max }=2$
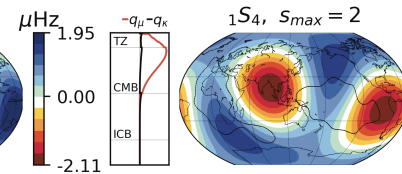

$\delta q_{\mu}=-0.2 \delta v_{s} / v$

Upper mantle 3D $q_{\mu}$
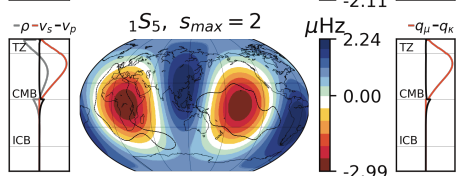

${ }_{1} S_{5}, S_{\max }=2$
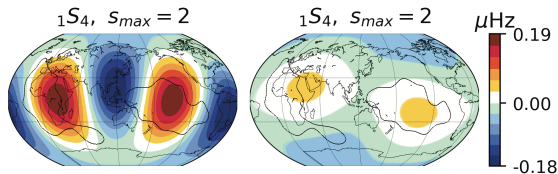

${ }_{1} S_{5}, S_{\max }=2$
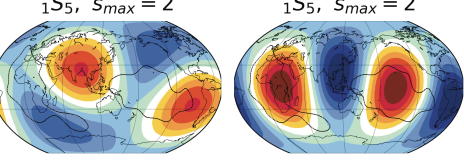

${ }_{1} S_{5}, S_{\max }=2 \quad \mu \mathrm{Hz}_{0.26}$

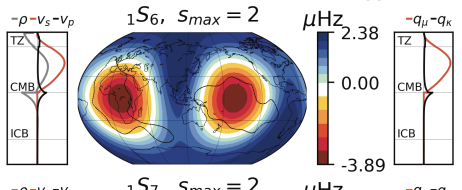

${ }_{1} S_{6}, S_{\max }=2$

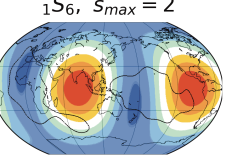

${ }_{1} S_{6}, S_{\max }=2$

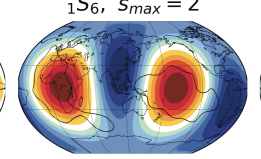

${ }_{1} S_{7}, S_{\max }=2$
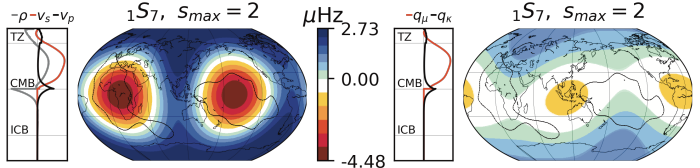

${ }_{1} S_{7}, S_{\max }=2$

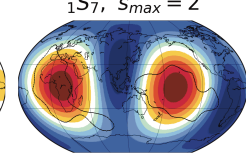

${ }_{1} S_{8}, S_{\max }=2$
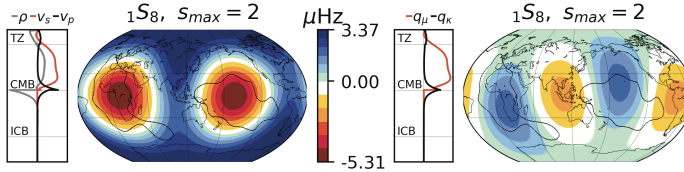

${ }_{1} S_{8}, S_{\max }=2$
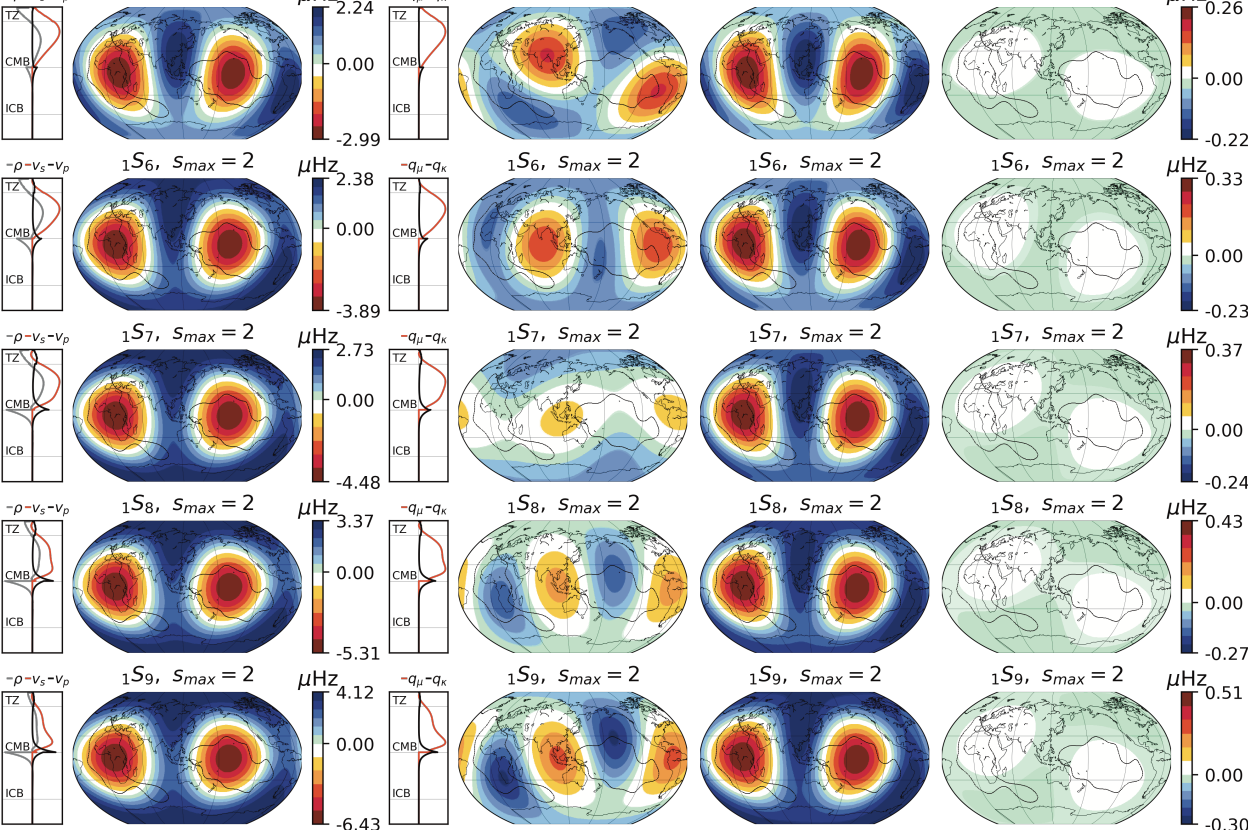

${ }_{1} S_{9}, S_{\max }=2$
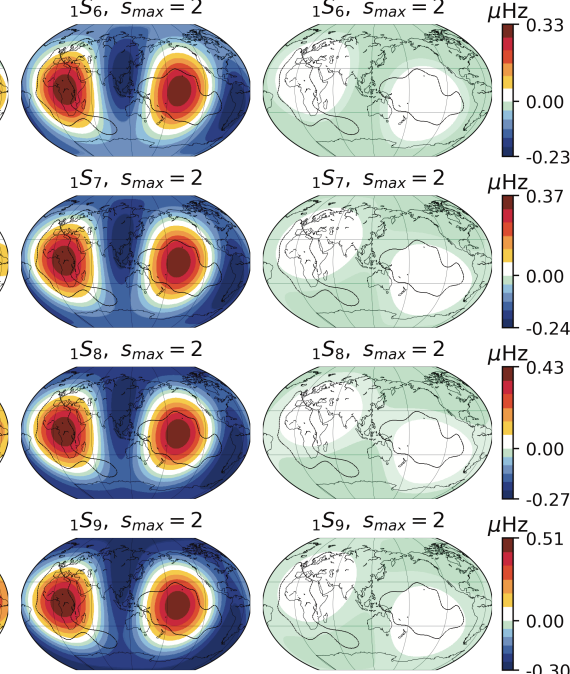

${ }_{1} \mathrm{~S}_{8}, S_{\max }=2 \quad \mu \mathrm{Hz}$
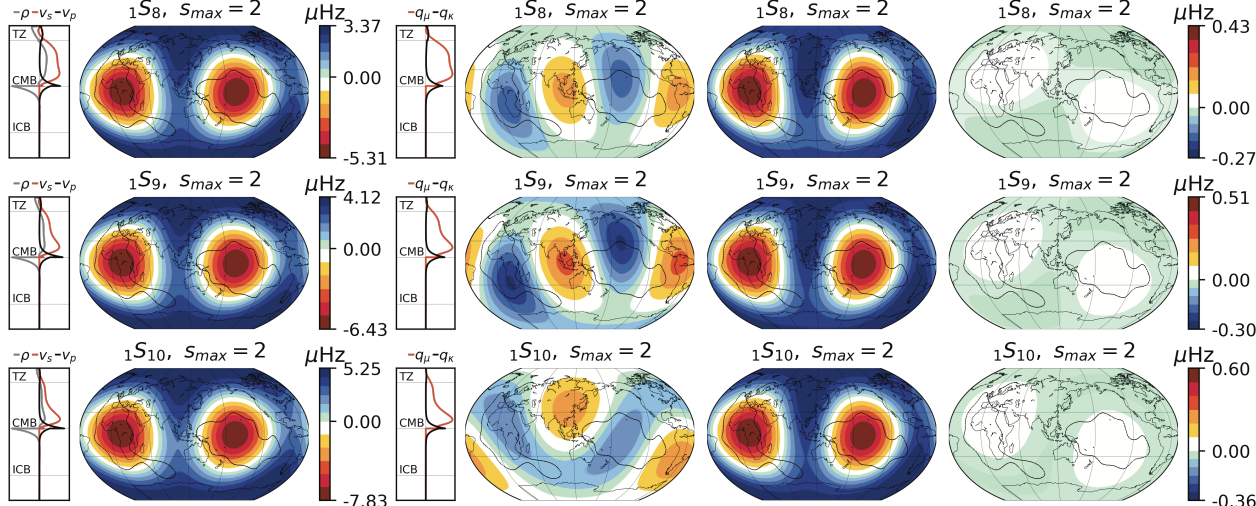

Figure 6.9: Splitting function measurements of first overtone modes ${ }_{1} S_{l}$ compared to mantle model predictions for $s_{\max }=2$, similar to Fig. 6.8 for $s_{\max }=2$.

find that these are significantly smaller than our $d_{s t}$ measurements. This is to be expected, because modes $n=0,1$ are mainly sensitive to the mid and lower mantle, while QRFSI12 only includes $q_{\mu}$ variations in the upper mantle. Nevertheless, the $d_{s t}$ predictions of QRFSI12 agree with the sign of the predictions of our global synthetic attenuation model anti-correlated to mantle $v_{s}$, and are opposite to our real data $d_{s t}$ measurements, which appear to be more correlated. The correlation between the $c_{s t}$ and $d_{s t}$ is also very apparent in the most dominant degree-two splitting functions coefficients. The real and imaginary parts of our measured $d_{22}$ coefficients for $n=0,1$ modes always have the same sign as our measured $c_{22}$ coefficients. They also 
Our $c_{s t}$ measurements

Our $d_{s t}$ measurements

S20RTS $d_{s t}$ predictions QRFSI12 $d_{\text {st }}$ predictions
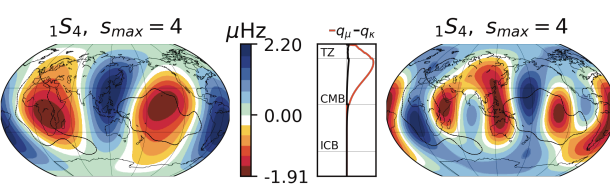

$\delta q_{\mu}=-0.2 \delta v_{s} / v$

Upper mantle 3D $q_{\mu}$

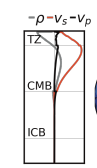

${ }_{1} S_{5}, S_{\max }=4$
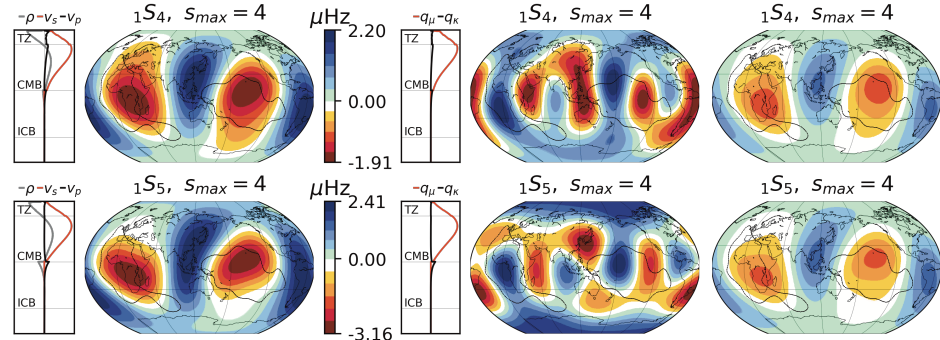

${ }_{1} S_{4}, S_{\max }=4$

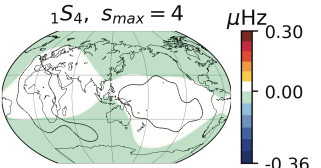

${ }_{1} S_{5}, S_{\text {max }}=4$
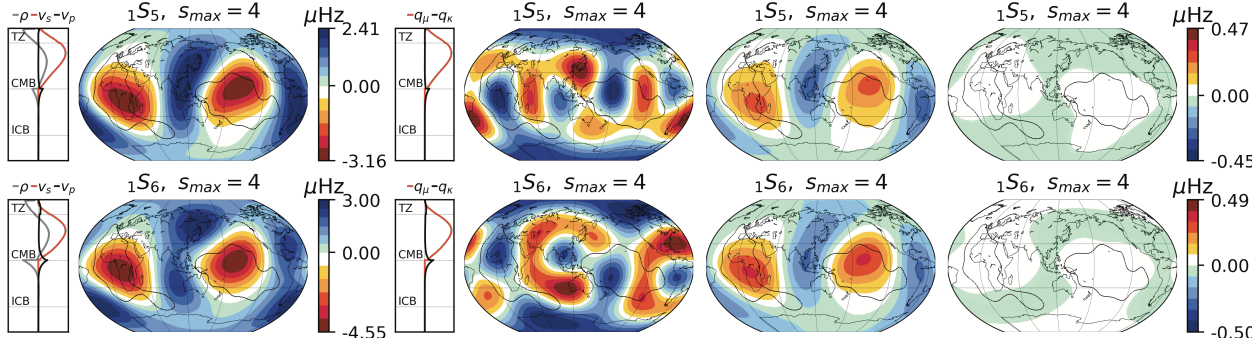

${ }_{1} S_{6}, S_{\max }=4$

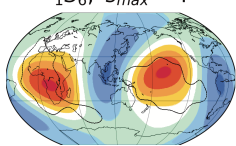

${ }_{1} S_{6}, S_{\max }=4 \quad \mu \mathrm{Hz}$
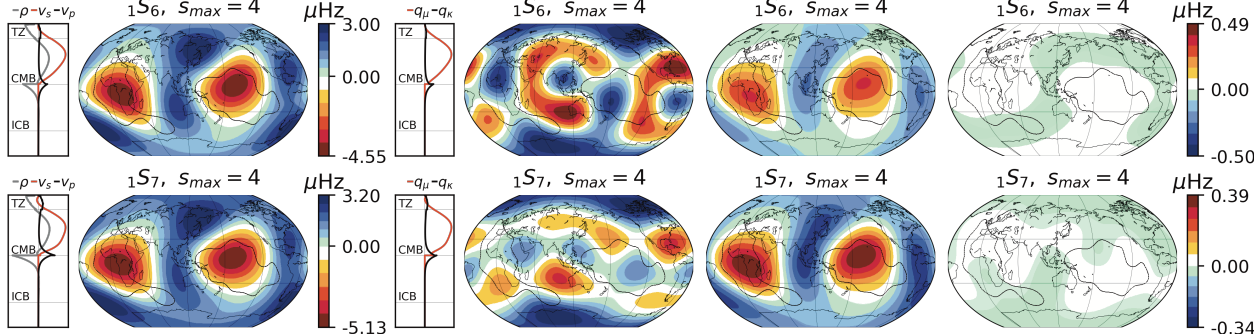

${ }_{1} S_{7}, S_{\max }=4$

${ }_{1} S_{7}, S_{\max }=4$
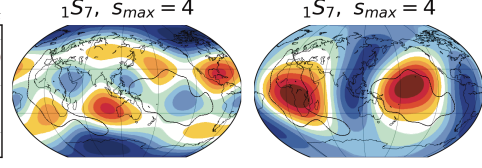

${ }_{1} \mathrm{~S}_{7}, S_{\max }=4 \quad \mu \mathrm{Hz}_{0.39}$
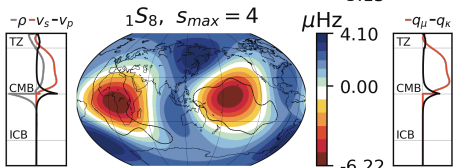

${ }_{1} S_{8}, S_{\max }=4$
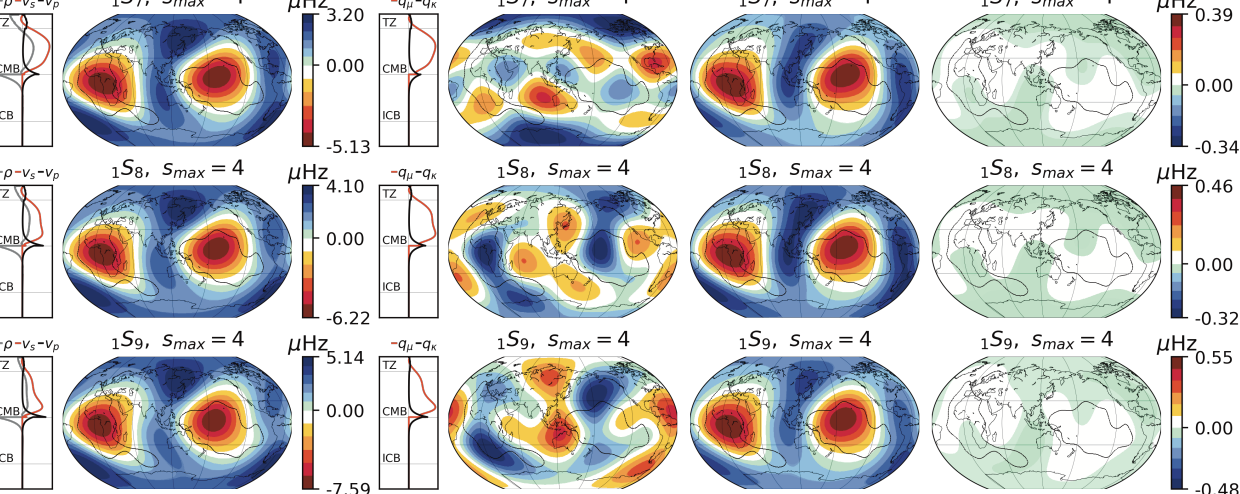

${ }_{1} \mathrm{~S}_{9}, \mathrm{~S}_{\max }=4 \quad \mu \mathrm{Hz}$
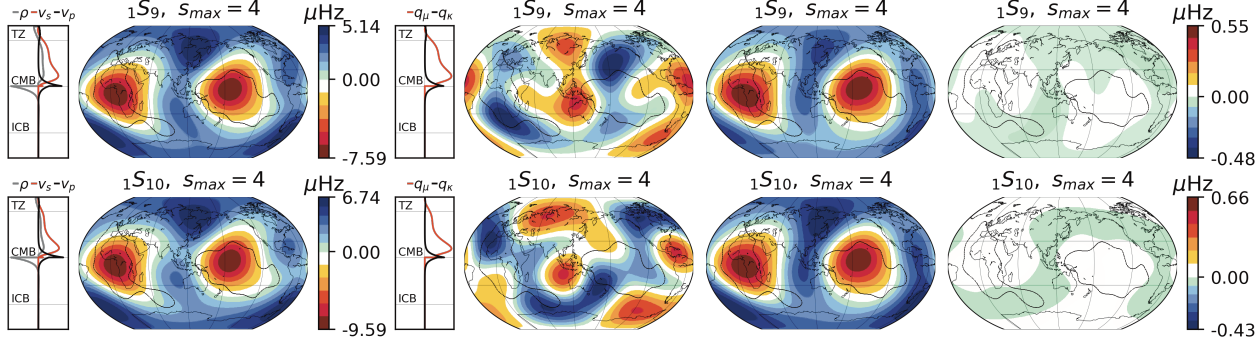

Figure 6.10: Splitting functions measurements of first overtone modes ${ }_{1} S_{l}$ compared to mantle model predictions for $s_{\max }=4$, similar to Fig. 6.8 for $s_{\max }=2$.

always have the opposite sign to the $d_{s t}$ coefficients predicted by our global synthetic attenuation model, in which variations in $q_{\mu}$ are anti-correlated to $v_{s}$ (Fig. 6.5).

As discussed in Section 5.3.2, mode branches ${ }_{0} S_{l}$ and ${ }_{1} S_{l}$ are also the most likely to potentially measure non-existent anelastic 'ghost' splitting function structure, as a result of trade-offs with errors in the scalar moment $M_{0}$ within the earthquake catalogue. With the exception of mode ${ }_{0} S_{2}$, the recovered 'ghost' signal in our tests for synthetic data is comparable in shape, but much smaller in size, than the $d_{s t}$ signal we measured using real data for the fundamental modes and first overtones modes sensitive to the mid mantle (e.g. ${ }_{1} S_{4},{ }_{1} S_{5},{ }_{1} S_{6},{ }_{1} S_{7}$ ). Both our $d_{s t}$ observations using real data, and the recovered synthetic $d_{s t}$, using a zero $3 \mathrm{D} q_{\mu}$ model combined 
with $M_{0}$ perturbations dependent on earthquake location, show what appears to be a correlation between anelastic and elastic splitting function coefficients, especially at degree-two (Figs. 6.4, 6.5, 6.B.4). However, the anelastic structure we observe for coefficients $\operatorname{Re}\left(d_{22}\right)$ and $\operatorname{Im}\left(d_{22}\right)$ using real data is 2 to 9 times larger than the 'ghost' structure in our tests (see Fig. 6.5). Furthermore, in our $M_{0}$ perturbation tests, the size of the recovered 'ghost' $d_{s t}$ structure stays roughly the same for all mid and lower mantle sensitive $n=0,1$ modes (see Figs. 6.4-6.5). In our $d_{s t}$ measurements, on the other hand, we see an increase in anelastic structure size as modes become more sensitive to the lower mantle (e.g. ${ }_{1} S_{8},{ }_{1} S_{9},{ }_{1} S_{10}$ ). For these reasons, we believe that our $d_{s t}$ measurements are robust, but their amplitudes might be affected due to trade-offs with errors in the earthquake moment magnitude.

\section{Mode ${ }_{0} S_{2}$}

The distinctive lower mantle sensitivity of mode ${ }_{0} S_{2}$ would in principle be extremely useful for studying lower mantle structure and is especially strongly sensitive to density (Koelemeijer et al., 2016; Moulik and Ekström, 2016; Akbarashrafi, 2020). However, unfortunately it has been notoriously difficult to measure ${ }_{0} S_{2}$ from normal mode spectra due to the lack of good quality data at the low frequency of this mode (Deuss et al., 2011). Here, we increased the amount of data used to measure mode ${ }_{0} S_{2}$ by including spectral segments for the $2011 M_{w} 9.1$ Tohoku earthquake. Even with the additional data, mode ${ }_{0} S_{2}$ still does not have enough data for its splitting functions to become well constrained. In our observations, the measured $Q$ value shift of the mode with respect to PREM flips sign with the inclusion of the Tohoku earthquake, becoming no longer branch consistent with the other fundamental modes (see Fig. 6.4 to compare our results to measurements of Deuss et al., 2013). Additionally, the measured anelastic signal of mode ${ }_{0} S_{2}$ is 10 times larger than the predicted anelastic signal for our global synthetic mantle attenuation model with $R_{q}=-0.2$ (Fig. 6.8). This is not what we see in the rest of our $d_{s t}$ observations for $n=0,1$ modes, which all are of similar size to the predicted anelastic splitting function coefficients for our synthetic 3D mantle attenuation model using the scaling $R_{q}=-0.2$.

Unlike other modes, which maintain a similar shape and sign for their sensitivity kernels for all their structural degrees, both the $v_{s}$ and $q_{\mu}$ sensitivity kernels of mode ${ }_{0} S_{2}$ flip sign, from positive to negative, between their degree-zero and degree-two sensitivity kernels (see Fig. 6.7 for the degree-zero $q_{\mu}$ kernel and Fig. 6.8 for the degree-two kernels). For this reason, we interpret the elastic splitting function of mode ${ }_{0} S_{2}$ using its degree-two elastic sensitivity kernels, instead of the degree-zero kernels, which are used for all other modes. This makes the interpretation of the elastic and anelastic splitting functions less straightforward than with other modes. As the degree-two $v_{s}$ sensitivity kernel of ${ }_{0} S_{2}$ is negative, we interpret the high frequency anomalies of its elastic splitting function as low velocity regions (Fig. 6.8). In the same manner, the degree-two $q_{\mu}$ sensitivity kernel of ${ }_{0} S_{2}$ is also negative, so we interpret the low frequencies anomalies of the anelastic splitting function as highly attenuating regions. This means mode ${ }_{0} S_{2}$ shows anti-correlation between elastic and anelastic structure, and disagrees with the $d_{s t}$ observations for the other fundamental 


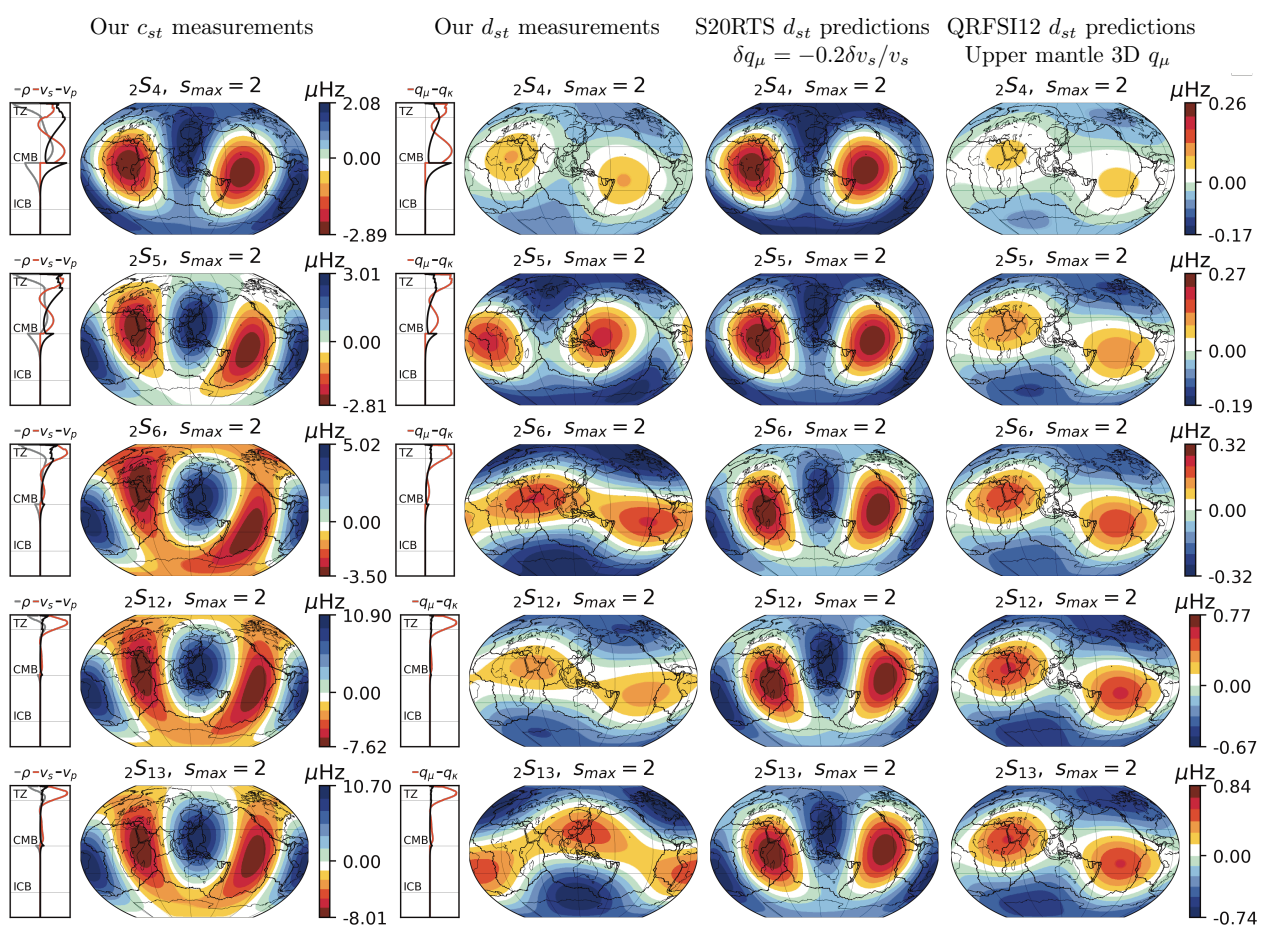

Figure 6.11: Splitting function measurements for second overtone ${ }_{2} S_{l}$ modes compared to mantle model predictions for $s_{\max }=2$. Tectonic plate boundaries (Bird, 2003) are included for comparison. See the caption of Fig. 6.8 for explanation.

modes (Fig. 6.8), as well as other lower mantle sensitive modes (e.g. ${ }_{1} S_{8},{ }_{1} S_{9},{ }_{1} S_{10}$ in Fig. 6.9). The same behaviour was seen in our $M_{0}$ tests (Section 5.3.2). In these tests, mode ${ }_{0} S_{2}$ showed larger frequency variations in the 'ghost structure' than other $n=0,1$ modes (Fig. 5.16). This is also what we see in our real data $d_{s t}$ measurements of ${ }_{0} S_{2}$, which are of the same order of magnitude as structure arising from errors in the scalar moment $M_{0}$. In addition, from full-coupling synthetics (Figs. 5.4-5.6) we know that modes below $1 \mathrm{mHz}$ are not very sensitive to the presence of $3 \mathrm{D}$ anelastic structure, which is especially the case for mode ${ }_{0} S_{2}$ at $0.3 \mathrm{mHz}$.

All of the above reasons, together with the low number of spectral segments available for this mode, leads us to believe that our anelastic splitting function measurement of ${ }_{0} S_{2}$ is not the result of actual mantle structure, but of the limited amount of spectral data available. Similar arguments have been used before to exclude this mode from lower mantle normal mode studies for $3 \mathrm{D}$ variations in density (Koelemeijer et al., 2017; Akbarashrafi, 2020). For these reasons, we will not include mode ${ }_{0} S_{2}$ in any further interpretation of our results (Section 6.4). 


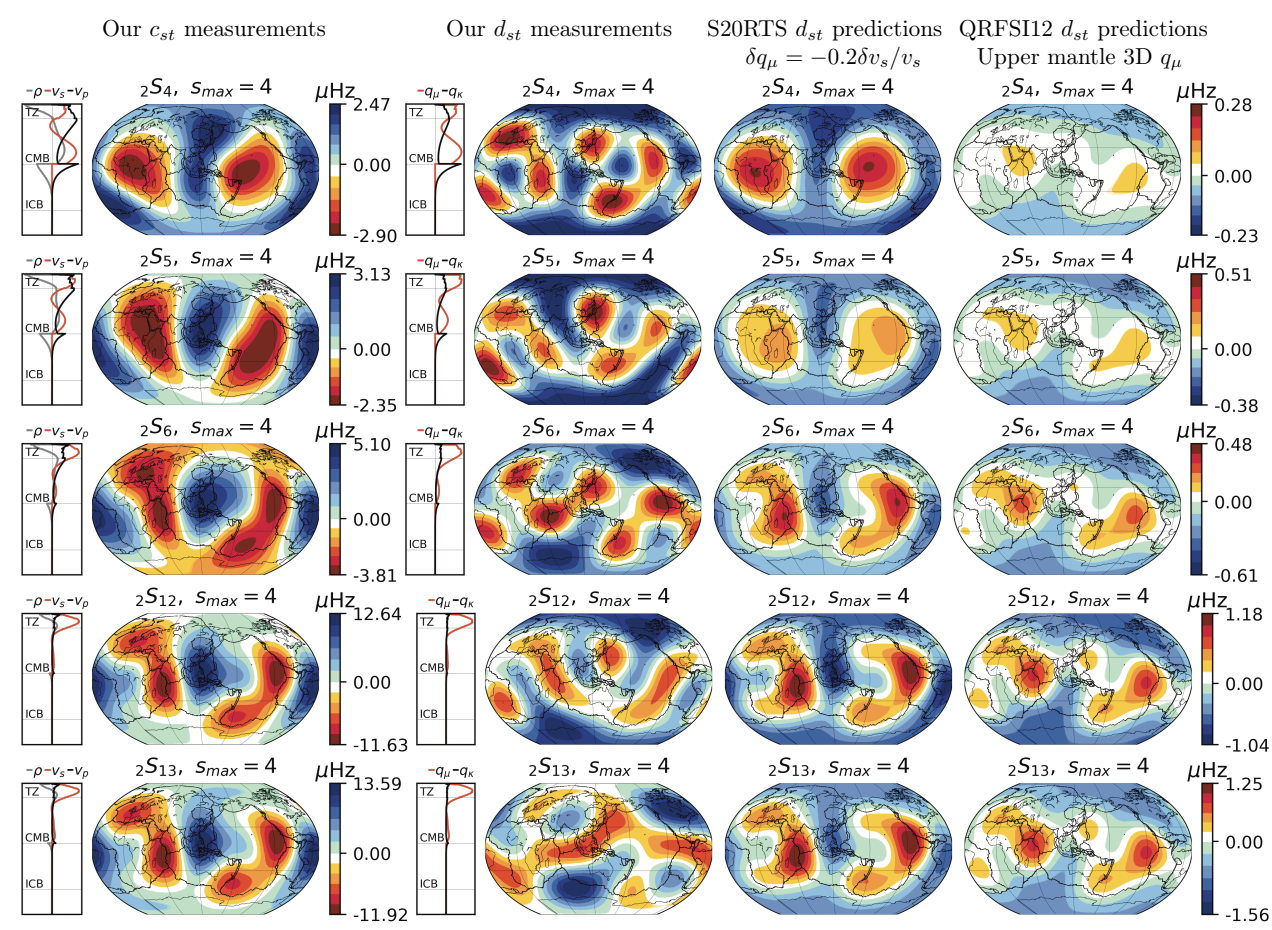

Figure 6.12: Splitting function measurements for second overtone ${ }_{2} S_{l}$ modes compared to mantle model predictions for $s_{\max }=4$, similar to Fig. 6.11 .

\subsection{2 $2^{\text {nd }}$ and $3^{\text {rd }}$ overtone modes}

Our anelastic splitting function measurements for the $n=2,3$ overtones which are mainly sensitive to the upper mantle, show the largest anelastic signal of all our modes. Again, this agrees with our synthetic forward modelling using $R_{q}=-0.2$ in Section 5.2.3, where modes mainly sensitive to the upper mantle showed the largest difference between synthetic full-coupling spectra with and without anelastic structure (Figs. 5.4-5.5). These modes also have the largest synthetic $d_{s t}$ values (i.e. ${ }_{2} S_{4},{ }_{2} S_{5},{ }_{2} S_{6},{ }_{2} S_{12},{ }_{2} S_{13},{ }_{3} S_{9}$ ). Splitting function measurements for the ${ }_{2} S_{l}$ and ${ }_{3} S_{9}$ modes for $s_{\max }=2,4,6$ are shown in Figs. 6.11-6.14. We use $s_{\max }=6$ as the maximum structural order for interpreting the $n=2,3$ overtones, which we validated by analysing the branch consistency of the measured $d_{s t}$. Mode ${ }_{2} S_{12}$ is the one exception; it is the only mode we were able to make stable measurement up-to degree $s_{\max }=10$ (Table 6.2).

All our ${ }_{2} S_{l},{ }_{3} S_{9}$ measurements are branch consistent and show strong and weak attenuation in the same regions (Figs. 6.11-6.14). Spheroidal $n=2,3$ modes, which are mainly sensitive to the upper mantle, show more anti-correlation between the elastic and anelastic splitting function measurements, unlike our measurements of $n=0,1$ modes. Especially at degree-two, the regions with the strongest attenuation 
Our $c_{s t}$ measurement

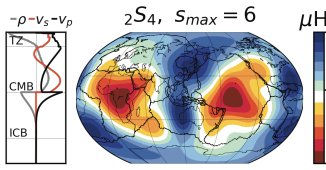

${ }_{2} S_{5}, S_{\max }=6$
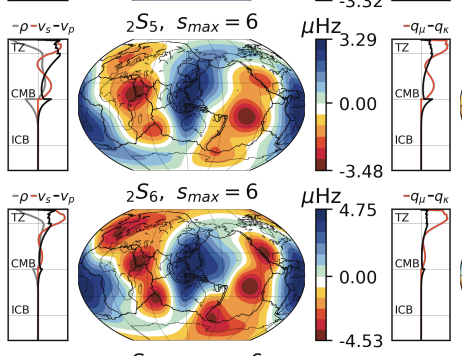

$-\rho-v_{5}-v_{p} \quad{ }_{2} S_{12}, S_{\max }=6 \quad \mu \mathrm{Hz}$

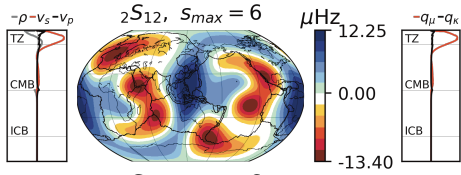

$-\rho-v_{s}-v_{0} \quad{ }_{2} S_{13}, S_{\max }=6 \quad \mu \mathrm{Hz}$

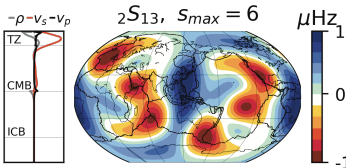

Our $d_{s t}$ measurements

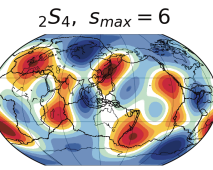

${ }_{2} S_{5}, S_{\max }=6$

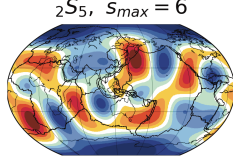

${ }_{2} S_{6}, S_{\max }=6$

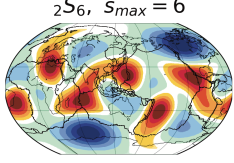

${ }_{2} S_{12}, S_{\max }=6$

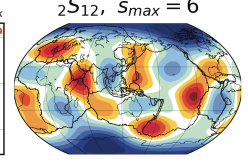

${ }_{2} S_{13}, S_{\max }=6$

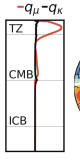

S20RTS $d_{s t}$ predictions QRFSI12 $d_{s t}$ predictions $\delta q_{\mu}=-0.2 \delta v_{s} / v_{s} \quad$ Upper mantle 3D $q_{\mu}$

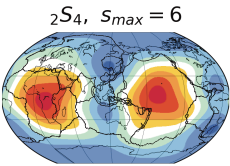

${ }_{2} S_{5}, S_{\max }=6$

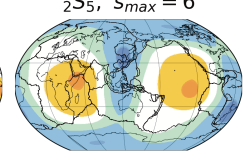

${ }_{2} S_{6}, S_{\max }=6$

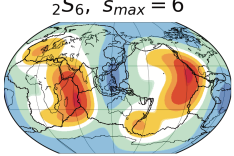

${ }_{2} S_{12}, S_{\max }=6$

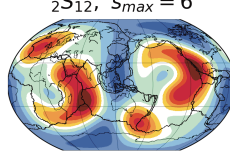

${ }_{2} S_{13}, S_{\max }=6$

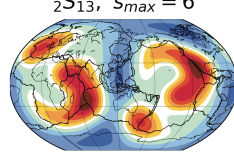

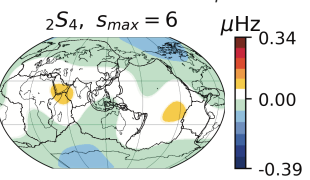

${ }_{2} S_{5}, S_{\text {max }}=6 \quad \mu \mathrm{Hz}$

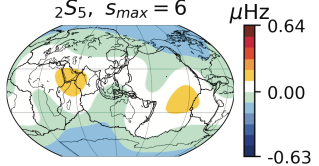

${ }_{2} S_{6}, S_{\max }=6 \quad \mu \mathrm{Hz}$

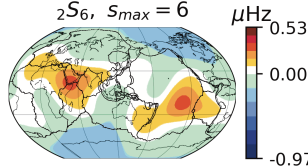

${ }_{2} S_{12}, S_{\max }=6 \quad$

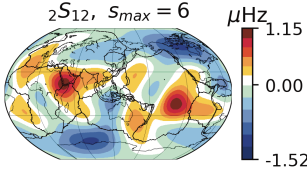

${ }_{2} S_{13}, S_{\max }=6 \quad \mu \mathrm{Hz}$

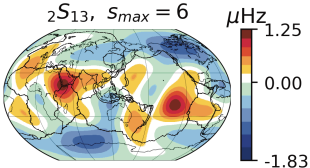

Figure 6.13: Splitting function measurements for the ${ }_{2} S_{l}$ branch compared to mantle model predictions for $s_{\max }=6$, similar to Fig. 6.11.

Our $c_{s t}$ measurements
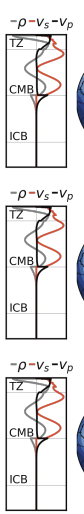

${ }_{3} S_{9}, S_{\max }=2$

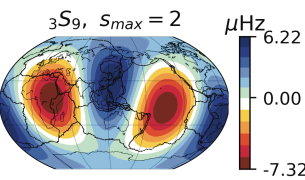

${ }_{3} S_{9}, S_{\max }=4$

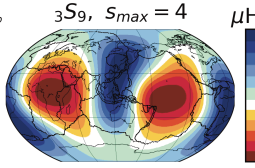

$S_{9}, s_{\max }=6$

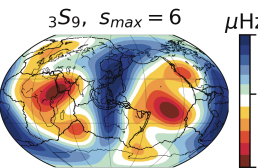

Our $d_{s t}$ measurements
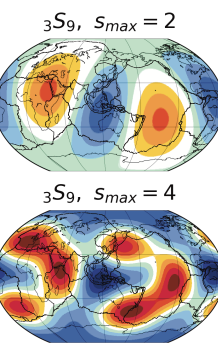

${ }_{3} S_{9}, S_{\max }=6$

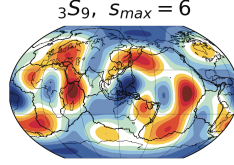

S20RTS $d_{s t}$ predictions QRFSI12 $d_{s t}$ predictions $\delta q_{\mu}=-0.2 \delta v_{s} / v_{s} \quad$ Upper mantle 3D $q_{\mu}$
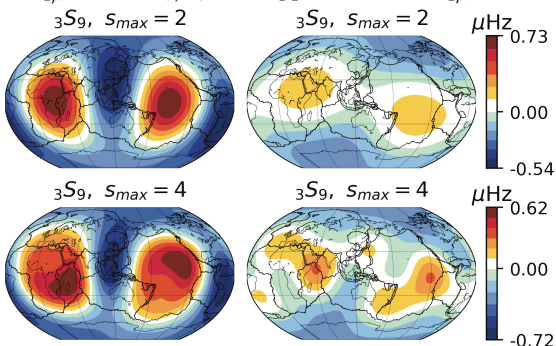

${ }_{3} S_{9}, S_{\max }=6$

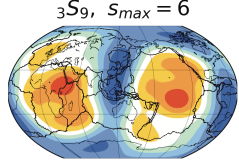

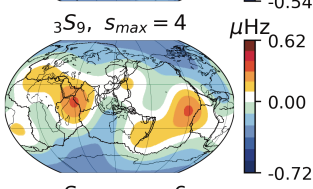

${ }_{3} S_{9}, S_{\max }=6 \quad \mu \mathrm{Hz}$

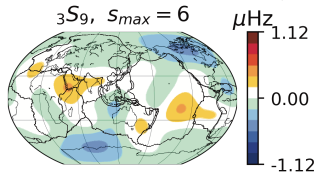

Figure 6.14: Splitting functions measurement for mode ${ }_{3} S_{9}$ compared to mantle model predictions for $s_{\max }=2,4,6$, similar to Fig. 6.11. 
in the anelastic splitting functions, which are visible in the east of Africa and the eastern Pacific, anti-correlate with low frequency anomalies in the elastic splitting functions (Figs. 6.11, 6.14). The anti-correlation between $c_{s t}$ and $d_{s t}$ structure for $n=2,3$ modes can also be seen in the most dominant splitting function coefficients $d_{20}$ and $\operatorname{Im}\left(d_{22}\right)$ (Figs. 6.4-6.5) The only exceptions are mode ${ }_{2} S_{5}$, which is also sensitive to the lower mantle, and mode ${ }_{2} S_{13}$, which is not as well constrained as our other $n=2,3$ modes. At higher degrees more details become visible and at $s_{\max }=6$ we see the most strongly attenuating regions close to mid-oceanic spreading ridges, including the southern part of the mid-Atlantic spreading ridge, the Indian Ocean ridge and the East Pacific Rise.

We also compare our measurements against the anelastic splitting function predictions for upper mantle attenuation model QRFSI12 (Dalton et al., 2008), as well as our global synthetic attenuation model in which the $q_{\mu}$ anomalies are anti-correlated to the $v_{s}$ anomalies of mantle model S20RTS by $R_{q}=-0.2$ (Figs. 6.11 6.14). QRFSI12 is an upper mantle model which decreases with depth and contains $q_{\mu}$ structure until $400 \mathrm{~km}$ depth. As a result, the QRFSI12 anelastic splitting function predictions have smaller amplitudes than our measurements, because even the most upper mantle sensitive modes analysed here are still sensitive to depths beyond $400 \mathrm{~km}$. The $d_{s t}$ predictions of both QRFSI12 and our synthetic 3D attenuation model are similar to our anelastic measurements for all ${ }_{2} S_{l}$ modes and ${ }_{3} S_{9}$, specially for degree-two. Our measured $d_{20}$ and $d_{22}$ coefficients also show in general the same sign and trend as predicted by both QRFSI12 and our synthetic attenuation model (Figs. 6.4-6.5).

Unlike the $n=0,1$ modes, which are significantly affected by $M_{0}$ perturbations dependent on earthquake location (Section 5.3.2), the $n=2$ modes show a recovered synthetic 'ghost' signal 10 times smaller than our actual anelastic splitting functions measurements using real data (Fig. 5.18). In addition, the recovered synthetic 'ghost' signal for $n=2$ modes in the $M_{0}$ test shows anelastic structure correlated to elastic structure, which is the opposite of what we see in our anelastic splitting functions measurements for real data and also in the QRFSI12 predictions (Dalton et al., 2008). For these reasons, we do not think that unaccounted $M_{0}$ perturbations have a significant effect on our measurements for the $n=2,3$ modes, and we think our anelastic splitting functions are robust.

\subsubsection{Cross-coupled splitting functions}

We also attempted to measure cross-coupled splitting functions for mode pairs ${ }_{1} S_{6^{-}}$ ${ }_{2} S_{5}$ and ${ }_{1} S_{5-2} S_{4}$. These mode pairs are cross-coupled for odd-degree structure and are sensitive to both the upper and lower mantle (Fig. 6.15). We made measurements for both mode pairs for degrees $s=1,3,5$. Our $d_{s t}$ measurements are branch consistent, and for mode pair ${ }_{1} S_{5-2} S_{4}$ agree with the shape of $d_{s t}$ prediction for QRFSI12 (Dalton et al., 2008). For mode pair ${ }_{1} S_{6-2} S_{5}$ the agreement with the QRFSI12 predictions is much less clear, which is surprising because the two modes pairs have such similar sensitivity kernels. So, both mode pairs are branch consistent in our real data measurements but not in the $d_{s t}$ predictions for QRFSI12 or our synthetic 3D attenuation model. We also note that degree-one structure dominates 
a) Our $c_{s t}$ measurements

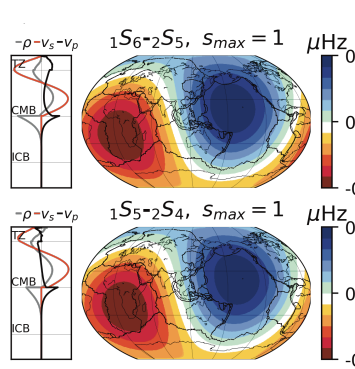

b) Our $c_{s t}$ measurements
Our $d_{s t}$ measurements

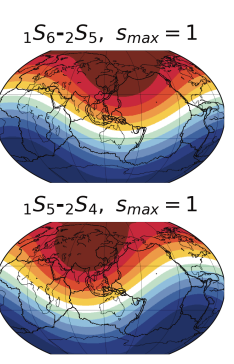

Our $d_{s t}$ measurements

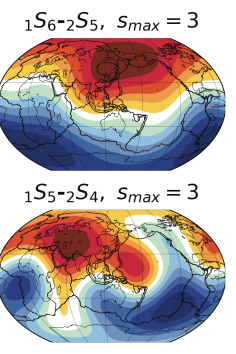

Our $d_{s t}$ measurements
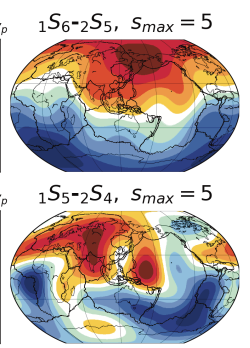

S20RTS $d_{s t}$ predictions QRFSI12 $d_{s t}$ predictions $\delta q_{\mu}=-0.2 \delta v_{s} / v_{s} \quad$ Upper mantle $3 \mathrm{D} q_{\mu}$
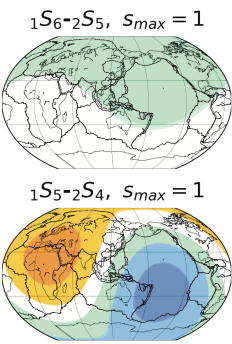

S20RTS $d_{s t}$ predictions $\delta q_{\mu}=-0.2 \delta v_{s} / v_{s}$ ${ }_{1} S_{6}-2 S_{5}, S_{\max }=3$

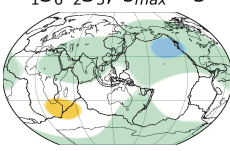

${ }_{1} S_{5-2} S_{4}, S_{\max }=3$

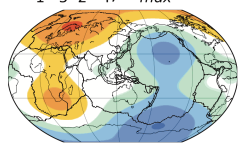

S20RTS $d_{s t}$ predictions $\delta q_{\mu}=-0.2 \delta v_{s} / v_{s}$ ${ }_{1} S_{6}-2 S_{5}, S_{\max }=5$
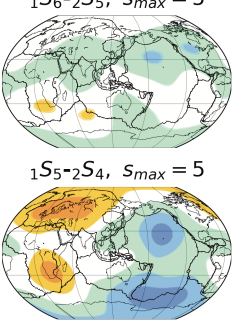

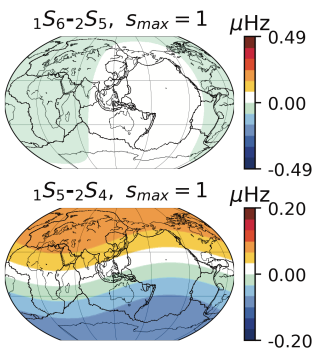

QRFSI12 $d_{s t}$ predictions Upper mantle 3D $q_{\mu}$

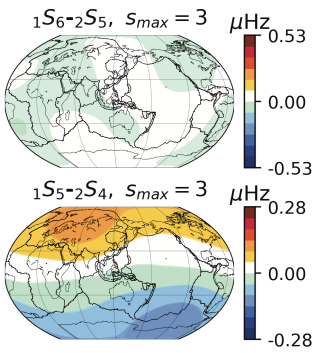

QRFSI12 $d_{s t}$ predictions Upper mantle 3D $q_{\mu}$

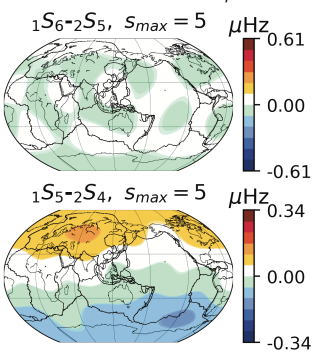

Figure 6.15: Splitting function measurements of ${ }_{1} S_{l^{-2}} S_{l-1}$ compared to mantle model predictions up-to degrees 1, 3, 5. See the caption of Fig. 6.11 for explanation.

over degrees 3 and 5 in the $d_{s t}$ for both mode pairs, while the $c_{s t}$ observations also show significant structure at degree 3. Finally, there is no clear correlation or anti-correlation relationship between the elastic and anelastic splitting functions.

Taking into consideration the large sensitivity of these cross-coupled splitting functions to the upper mantle, we assume that the upper mantle signal will dominate over any lower mantle signal reflected in these measurements. If this assumption is indeed correct, then the changes in frequency in these measurements should be explained in the opposite way as our self-coupling measurements, given that their sensitivity kernels are opposite in sign, i.e. high frequency anomalies relate to low velocity or weak attenuation. However, given the complicated cross-coupling sensitivity kernels, which are sensitive to both the upper and lower mantle and 
flip sign in the transition zone, interpreting these measurements in terms of 3D attenuation variations is not possible without constructing a $3 \mathrm{D}$ attenuation model using tomographic inversion. Still, it is encouraging to see that our anelastic splitting function observations agree in shape and sign with the strong $s=1$ structure that is also present in the QRFSI12 splitting function prediction (Dalton et al., 2008).

\subsection{Interpretation}

We would like to interpret our elastic and anelastic splitting functions observations in terms of mantle thermal and compositional structure. But without performing an actual tomographic inversion, we will need to make some simplifying assumptions in order to interpret our observed elastic and anelastic splitting functions, and to compare them to currently existing tomographic velocity and attenuation models. Based on the sensitivity kernels of our measured modes, we assume that all elastic anomalies $c_{s t}$ are mainly due to shear velocity $v_{s}$ and not compressional velocity $v_{p}$ or density $\rho$, and that all anelastic anomalies $d_{s t}$ are mainly due to shear attenuation $q_{\mu}$ and not bulk attenuation $q_{\kappa}$. In this way, we interpret our elastic and anelastic splitting function observations as showing either correlation or anti-correlation between $v_{s}$ and $q_{\mu}$ structure. We realise that it would be much better to perform an actual tomographic inversion of the $d_{s t}$ and make a $q_{\mu}$ model, which will be the subject of future research. In order to compare our $d_{s t}$ observations to upper mantle $q_{\mu}$ model QRFSI12 (Dalton et al., 2008), we plot QRFSI12 for even-degree structure up-to degree $s_{\max }=6$ in Fig. 6.16 .

In this section, instead of interpreting our normal mode measurements as a function of their overtone number $n$, we will interpret them as a function of their $q_{\mu}$ sensitivity to the upper, mid or lower mantle as classified in Fig. 6.7.

\subsubsection{Upper mantle}

\subsubsection{Comparison with existing $v_{s}$ and $q_{\mu}$ mantle models}

Spheroidal modes ${ }_{2} S_{6},{ }_{2} S_{12}$ and ${ }_{2} S_{13}$ are mainly sensitive to the upper mantle; modes ${ }_{2} S_{4},{ }_{2} S_{5}$ and ${ }_{3} S_{9}$ are also predominantly sensitive to the upper mantle but also have some lower mantle sensitivity. All these modes show an anti-correlation between the elastic and anelastic splitting function measurements (see Section 6.3.2). Our most robust observation is for mode ${ }_{2} S_{12}$, which will be the main focus of the following interpretation.

Our elastic splitting function observation of mode ${ }_{2} S_{12}$, shows positive frequency anomalies in shield areas and subduction zone regions and negative frequency anomalies along mid-oceanic spreading ridges (Fig. 6.17a). Our observations are in agreement with predictions for mantle shear wave velocity model S40RTS (Ritsema et al., 2011) confirming that the low frequency anomalies in the ridges are indeed due to low velocity (Fig. 6.17b). Our anelastic splitting function of ${ }_{2} S_{12}$ shows positive frequency anomalies along mid-oceanic spreading ridges (e.g. the mid-Atlantic, central Indian, and Pacific-Antarctic ridge and the East Pacific Rise), which are due to strong $q_{\mu}$ attenuation (Fig. 6.17c). Some of these ridges also show strong attenuation in the upper mantle 3D attenuation model of Dalton et al., 2008 (Fig. 6.17d). Thus, for the 

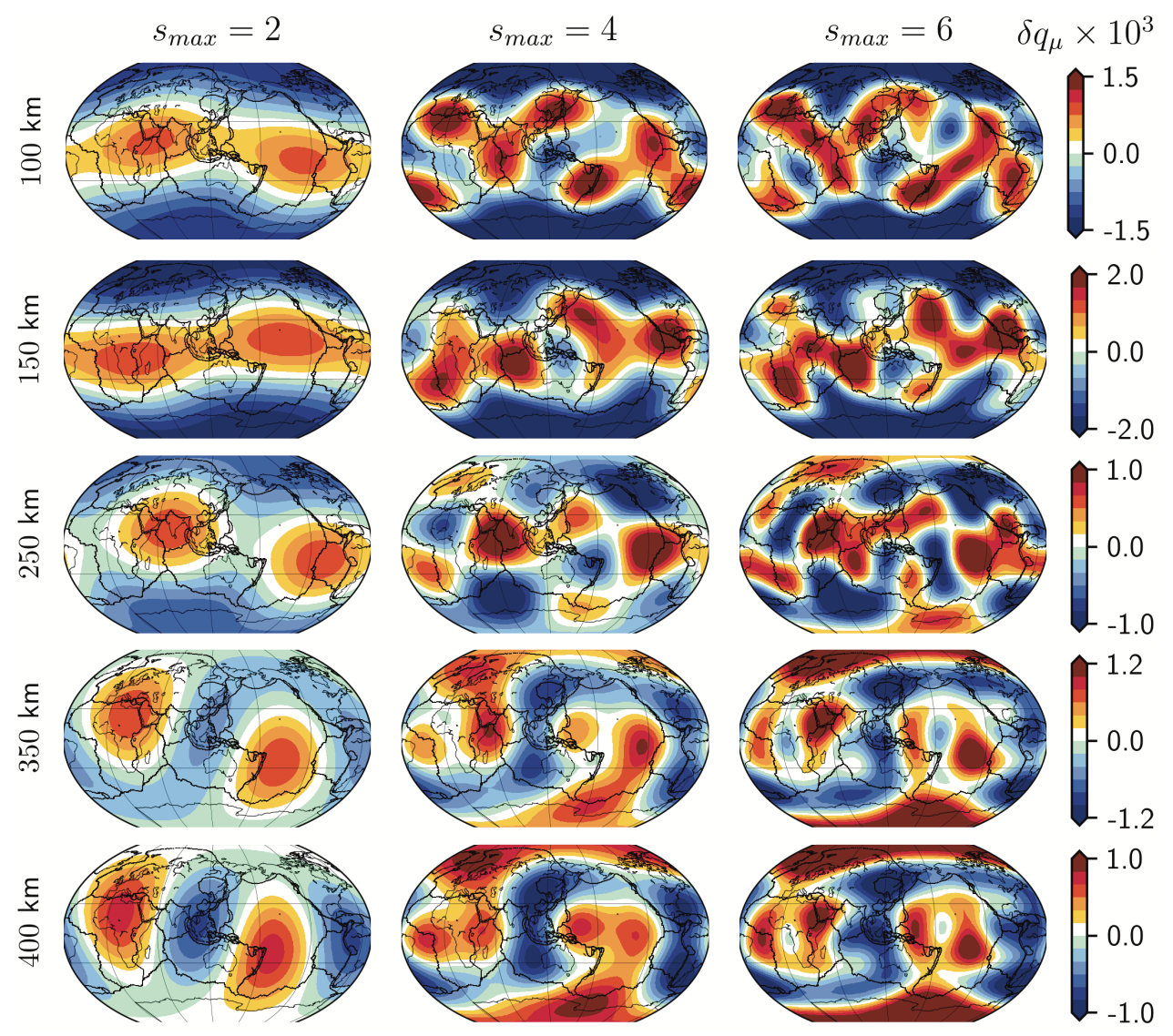

Figure 6.16: 3D $q_{\mu}$ upper mantle models QRFSI12 (Dalton et al., 2008) plotted for evendegree structure for $s_{\max }=2,4,6$ at $100 \mathrm{~km}, 150 \mathrm{~km} 250 \mathrm{~km}, 350 \mathrm{~km}$ and $400 \mathrm{~km}$ depth. For comparison with self-coupling splitting functions, that can only measure even-degree structure. Tectonic plate boundaries (Bird, 2003) are included.

upper mantle we agree with previous studies (Dalton et al., 2008; Zhu et al., 2013; Karaoğlu and Romanowicz, 2018) and find anti-correlation between shear velocity $v_{s}$ and attenuation $q_{\mu}$. This means that regions with high $q_{\mu}$, or strong attenuation, are found in places where we have low $v_{s}$ anomalies.

The anelastic splitting functions of our upper mantle sensitive $n=2,3$ modes show a dominant degree-two structure (Figs. 6.11, 6.14), which is also seen in the upper mantle $q_{\mu}$ models shown in Fig. 6.2. It is interesting to note that our upper mantle sensitive modes show a larger than expected negative $d_{20}$ coefficients, especially when compared to our global synthetic attenuation model obtained by anti-correlating $q_{\mu}$ with $v_{s}$ mantle model S20RTS by $R_{q}=-0.2$ (Fig. 6.4). This behaviour was also seen by Romanowicz et al. (1987) and Roult et al. (1990), who measured upper mantle sensitive spheroidal fundamental modes of angular order 
a) Our $c_{s t}$ measurements
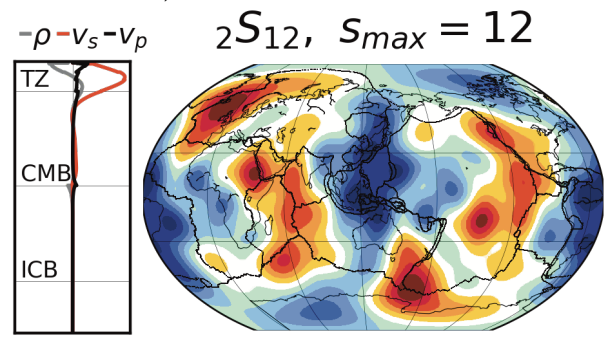

c) Our $d_{s t}$ measurements

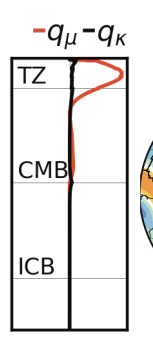

b) S40RTS $c_{s t}$ predictions

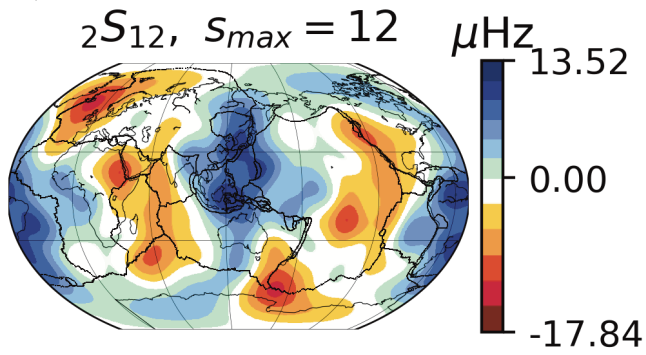

d) QRFSI12 $d_{s t}$ predictions

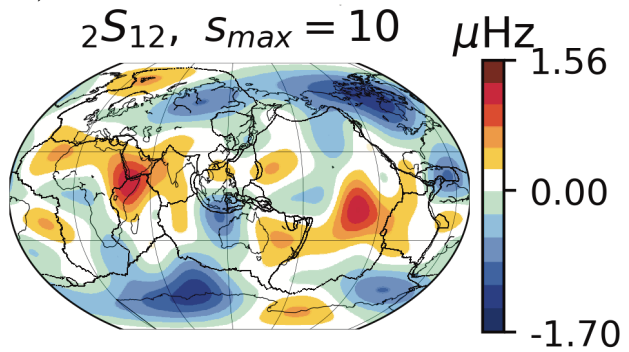

Figure 6.17: Splitting function maps for upper mantle sensitive mode ${ }_{2} S_{12}$ plotted together with its respective sensitivity kernels for $v_{s}$ and $q_{\mu}$ (red lines), $v_{p}$ and $q_{\kappa}$ (black lines) and $\rho$ (grey line). (a) Elastic $c_{s t}$ measurements compared to (b) the $c_{s t}$ predictions for $v_{s}$ mantle model S40RTS (Ritsema et al., 2011) together with crustal model CRUST5.1 (Mooney et al., 1998); and (c) anelastic $d_{s t}$ measurements compared to (d) the $d_{s t}$ predictions for the upper mantle model QRFSI12 (up-to $400 \mathrm{~km}$, Dalton et al., 2008). Tectonic plate boundaries (Bird, 2003) are also included for comparison.

$l=10-65(2.8-6.5 \mathrm{mHz})$, and found that coefficients $d_{20}$ and $\operatorname{Im}\left(d_{22}\right)$ dominate the anelastic structure in the upper mantle. They linked the anomalously large and negative $d_{20}$ to potentially unaccounted excess ellipticity. It is important to highlight that in these studies, due to data constrains (maximum 130 vertical seismic records), the anelasticity is measured by averaging the $q$ of modes over three to five angular orders. Nevertheless, we do agree that excess ellipticity may also be visible in our anelastic splitting function observations.

Our anelastic splitting functions at degree 4 and 6 (Figs. 6.12, 6.14) of ${ }_{2} S_{l}$ modes and ${ }_{3} S_{9}$, show similar high attenuation regions in the Pacific and central Indian ocean as seen at $100 \mathrm{~km}$ depth in model QRFSI12 (Dalton et al., 2008) for the same degrees (Fig. 6.16). Our anelastic measurements also agree with our global synthetic attenuation model anti-correlated to the $v_{s}$ mantle model S20RTS with $R_{q}=-0.2$, which assumes all attenuation variations are a result of temperature variations. It is important to note, however, that in our measurements we also see tectonically complex areas, such as back-arc spreading centers, which show correlation between strong attenuation and fast velocities (such as near the Japan, Mariana, Fiji and Manus trenches). 

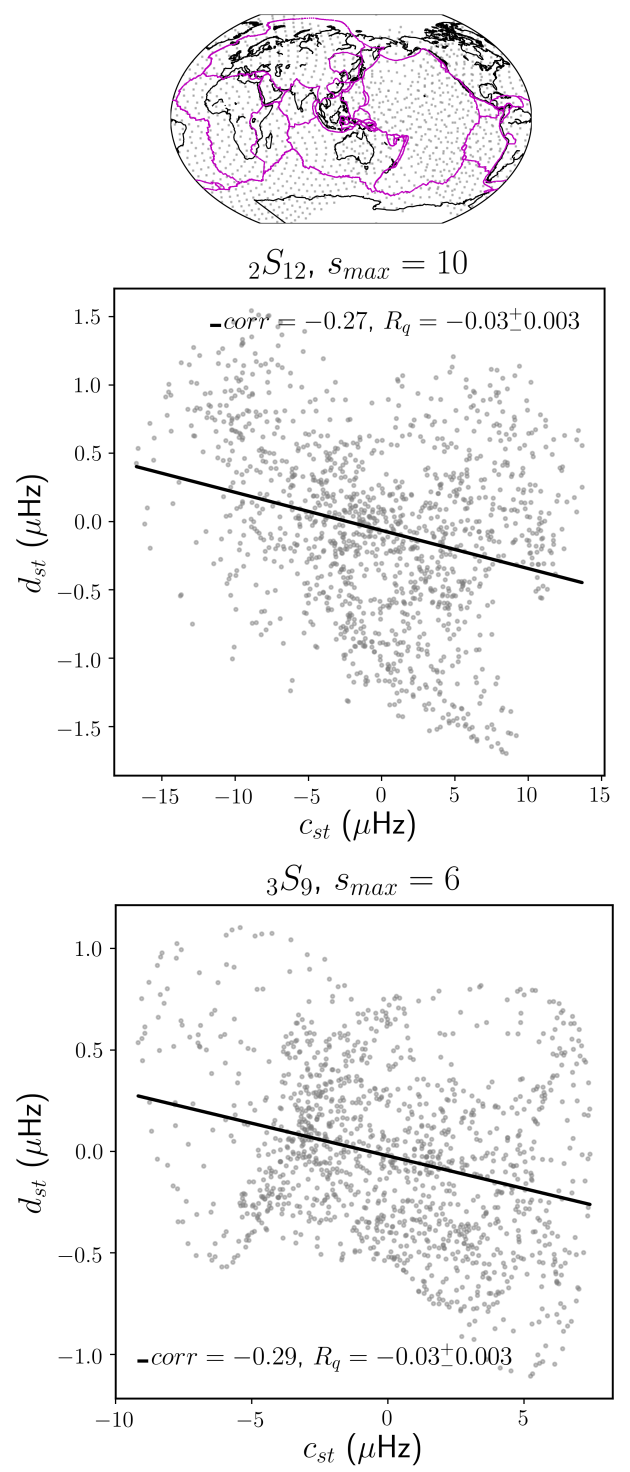
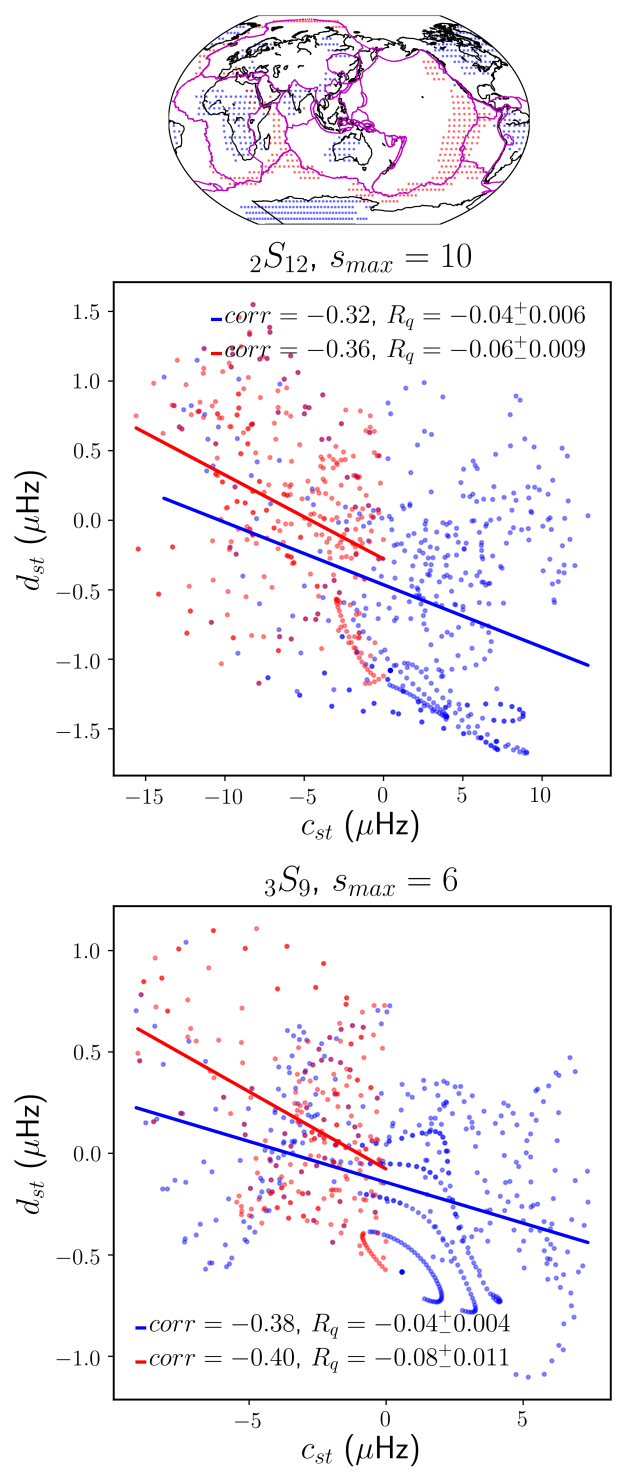

Figure 6.18: $c_{s t}$ versus $d_{s t}$ splitting function map values compared for upper mantle sensitive modes ${ }_{2} S_{12}$ and ${ }_{3} S_{9}$. (left) Each grey point (1300) represents an equidistant latitude and longitude location in the $c_{s t}$ and $d_{s t}$ splitting function maps. (right) Regional variations for cratons (blue) and young oceans ( $<20$ million years) (red). A simple linear regression is calculated, where corr is the correlation and $R_{q}$ is the slope. See Fig. 5.A.3 to compare $R_{q}$ in our observations to the one using S20RTS (Ritsema et al., 1999) predictions. 
a) Our $c_{s t}$ measurements

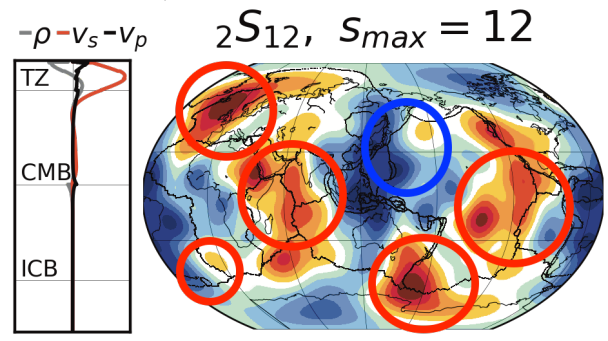

c) Our $d_{s t}$ measurements

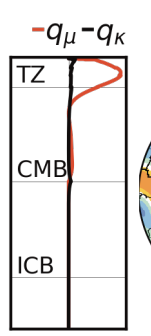

b) S40RTS $c_{s t}$ predictions

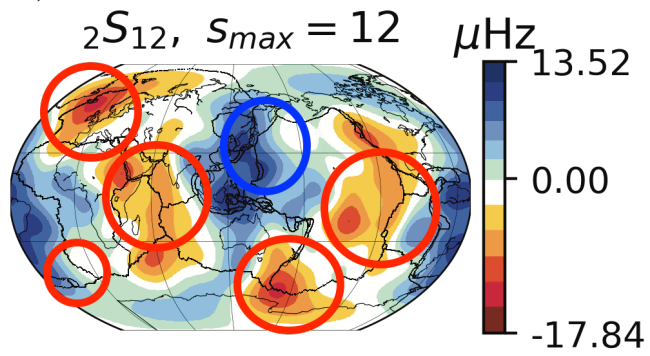

d) QRFSI12 $d_{s t}$ predictions

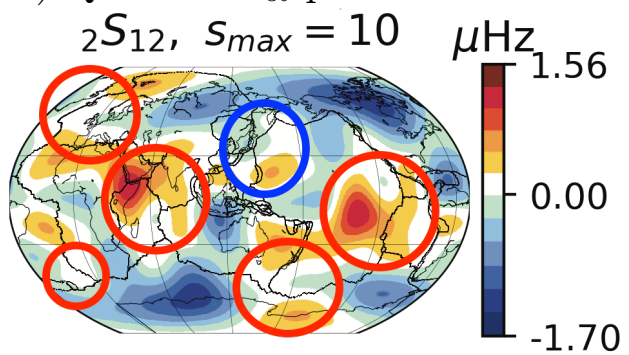

Figure 6.19: Highlighted version of Fig. 6.17, regions showing anti-correlation between elastic and anelastic structure in red circles, correlation in blue circles. See the caption of Fig. 6.17 for explanation.

We have shown that our elastic and anelastic splitting function observations appear to be anti-correlated for upper mantle sensitive modes. In order to investigate this further, we would like to quantify the level of anti-correlation between our elastic and anelastic upper mantle measurements. This is achieved by plotting our $c_{s t}$ and $d_{s t}$ frequency variations from our splitting function maps against each other, in a similar way as in Fig. 6.1, for equidistant latitude and longitude points (Fig. 6.18). We then calculate the anti-correlation level by fitting the point cloud with a simple linear relationship. For modes ${ }_{2} S_{12}$ and ${ }_{3} S_{9}$ we find a correlation factor between elastic and anelastic structure of -0.27 and -0.29 respectively, confirming the anti-correlation we inferred by comparing our $c_{s t}$ and $d_{s t}$ observations by eye (see Figs. 5.A.2-5.A.3 for comparison).

The amount of anti-correlation depends on the tectonic region, and we see that the globally averaged anti-correlation value is lowered by tectonically complex regions, such as back-arcs which show correlation instead of anti-correlation in our measurements. The excess ellipticity in our anelastic measurements (i.e. larger than expected $d_{20}$ ) also lowers the global level of anti-correlation between elastic and anelastic structure for upper mantle sensitive modes. Therefore, we also performed the same correlation analysis by separating our splitting function maps into regions associated to cratons and young oceans ( $<20$ million years), which show higher anti-correlation values, between -0.32 and -0.38 for cratons and -0.36 and -0.4 for young oceans, than the globally averaged values of -0.27 and -0.29 (Fig. 6.18). 
The largest anti-correlation values are found for the young oceans, in agreement with their proximity to the spreading ridges and their predominantly thermal origin and relatively high temperature.

\subsubsection{Mineralogical interpretation}

Experimental deformation studies on the upper mantle mineral olivine have found that increasing temperature lowers the shear modulus $\mu$ and increases attenuation $q_{\mu}$ (Faul and Jackson, 2005). Thus, our observed anti-correlation between the elastic splitting function (assumed to be due to shear wave velocity variations) and anelastic splitting function (due to attenuation variations), indicates that upper mantle anomalies have a thermal origin. Our long-period normal mode observations are in agreement with strong attenuation found beneath spreading ridges and weak attenuation in shield regions, confirming previous studies using shorter period surface wave data and body waves (e.g. Romanowicz and Gung, 2002; Dalton et al., 2008).

In some regions, our splitting function measurements show correlation between the elastic and anelastic splitting functions instead, particularly in back-arcs regions (e.g. near the Japan, Mariana, Fiji and Manus trenches) where strong attenuation and fast velocities are seen. This correlation could potentially indicate the presence of water, which is expected to have a larger effect on attenuation than on seismic velocity (Karato and Jung, 1998; Karato, 2003; Aizawa et al., 2008; Lee, 2003). Water presence in back-arcs spreading centers can be explained by the subducting oceanic plate carrying down water into the subduction zone, which is released later into the back-arc in the overriding plate.

\subsubsection{Lower mantle}

\subsubsection{Comparison with existing $v_{s}$ and $q_{\mu}$ mantle models}

Next, we move from the upper mantle sensitive modes to the lower mantle sensitive modes, which includes our fundamental and $1^{\text {st }}$ overtone measurements (see Section 6.3.1). Our most robust lowermost mantle sensitive mode observations are for the $1^{\text {st }}$ overtones ${ }_{1} S_{8},{ }_{1} S_{9}$ and ${ }_{1} S_{10}$, and these modes will be the main focus our interpretation below, because they are the most relevant to answer questions regarding the nature of the LLSVPs. Fundamental modes ${ }_{0} S_{5},{ }_{0} S_{6}$ and ${ }_{0} S_{7}$ are also sensitive to the lower mantle, but their observations are less robust. The remaining $1^{\text {st }}$ overtones ${ }_{1} S_{4},{ }_{1} S_{5},{ }_{1} S_{6}$ and ${ }_{1} S_{7}$ are mainly sensitive to the mid-mantle.

The relationship between our elastic and anelastic splitting function observations changes from the upper to the lower mantle. We will use mode ${ }_{1} S_{9}$ as our main example in the discussion below. Our elastic splitting function observation for lowermost mantle sensitive mode ${ }_{1} S_{9}$, shows negative frequency anomalies in the areas associated with the LLSVPs, which in turn are surrounded by positive frequency anomalies (Fig. 6.20a). This is the characteristic 'ring around the Pacific' structure which dominates lower mantle elastic seismic structure and is also present in elastic splitting function predictions for shear wave velocity model S40RTS (Fig. 6.20b). This structure is clearly visible at $s_{\max }=2$, with the dominant degree-two signal still visible even when including larger structural degrees (up-to 10 or 12). 
a) Our $c_{s t}$ measurements

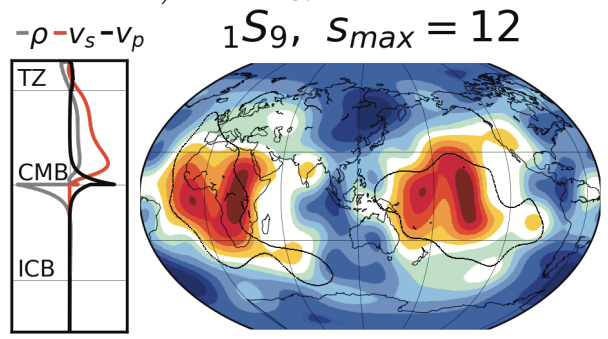

c) Our $d_{s t}$ measurements

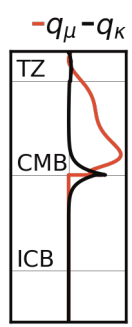

b) S40RTS $c_{s t}$ predictions

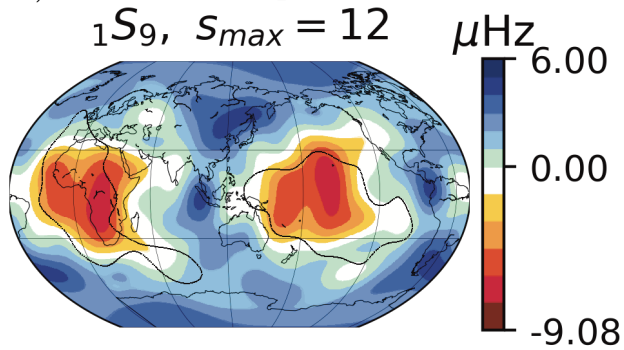

d) QRFSI12 $d_{s t}$ predictions

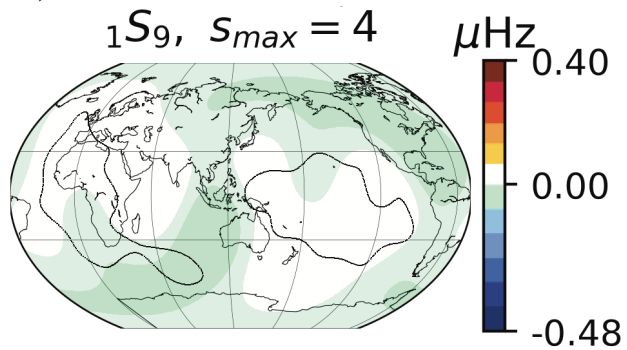

Figure 6.20: Lower mantle mode ${ }_{1} S_{9}$. The $-0.1 \% v_{s}$ outline of the tomographic model SP12RTS at $2850 \mathrm{~km}$ (Koelemeijer et al., 2016), which contours the LLSVPs, is included for comparison. See the caption of Fig. 6.17 for explanation.

The anelastic splitting functions for degree-two structure of lower mantle sensitive modes also show negative frequency anomalies in the LLSVP regions, and positive anomalies in the surrounding regions (e.g. ${ }_{0} S_{5},{ }_{0} S_{6},{ }_{0} S_{7},{ }_{1} S_{8},{ }_{1} S_{9},{ }_{1} S_{10}$ in Figs. 6.86.9). However, when degree-four structure is included, the anelastic splitting functions show more regional variations instead of just the LLSVPs, which are the dominant signal in the elastic splitting function also at higher degrees. In the anelastic splitting functions, parts of the LLSVPs have weak attenuation, but some other parts, especially the edges, have stronger attenuation. This implies that the LLSVPs are not completely thermal regions, and require compositional heterogeneity, in agreement with studies on 3D density variation of the LLSVPs, which we will discuss in more detail in the next section. For mode ${ }_{1} S_{9}$, the strongest attenuation appears under Oceania and central America, with an additional region of strong attenuation under North East Asia and South America (Fig. 6.20c).

Unlike upper mantle sensitive $n=2,3$ modes, which are dominated by an anomalously large $d_{20}$ coefficient, the lower mantle sensitive $n=0,1$ modes are dominated by the $\operatorname{Re}\left(d_{22}\right)$ coefficient (e.g. ${ }_{0} S_{5},{ }_{0} S_{6},{ }_{0} S_{7},{ }_{1} S_{8},{ }_{1} S_{9},{ }_{1} S_{10}$ in Fig. 6.5). These negative $\operatorname{Re}\left(d_{22}\right)$ coefficients result in low frequency regions (i.e. weak attenuation) under the Pacific and Africa, where the LLSVPs are located; and high frequency zones (strong attenuation) in the regions surrounding the LLSVPs (Figs. 6.8, 6.9, 6.5).

It is interesting to note, that modes ${ }_{1} S_{4},{ }_{1} S_{5},{ }_{1} S_{6}$ and ${ }_{1} S_{7}$, which are mostly sensitive to the mid mantle, show degree-four structure that resembles our measurements 

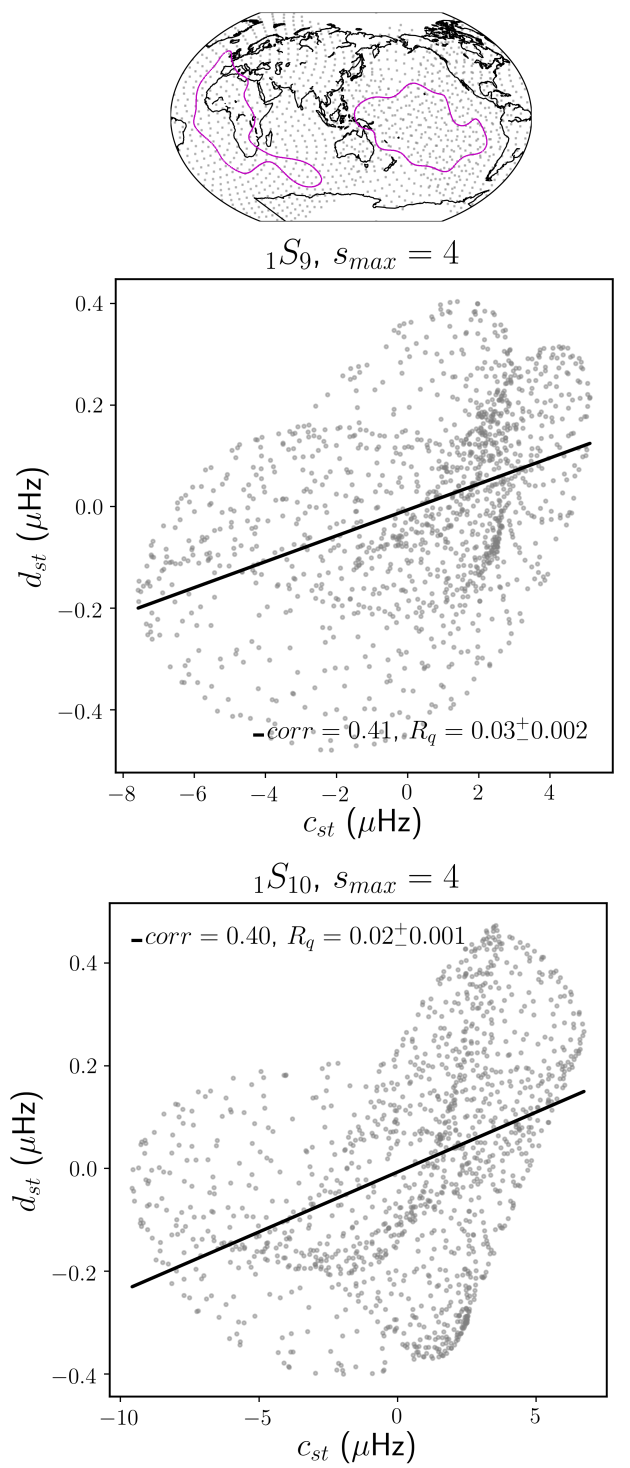

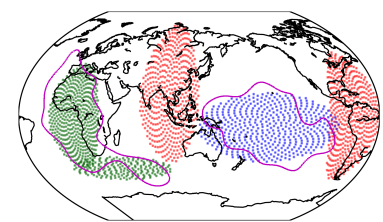

${ }_{1} S_{9}, s_{\max }=4$
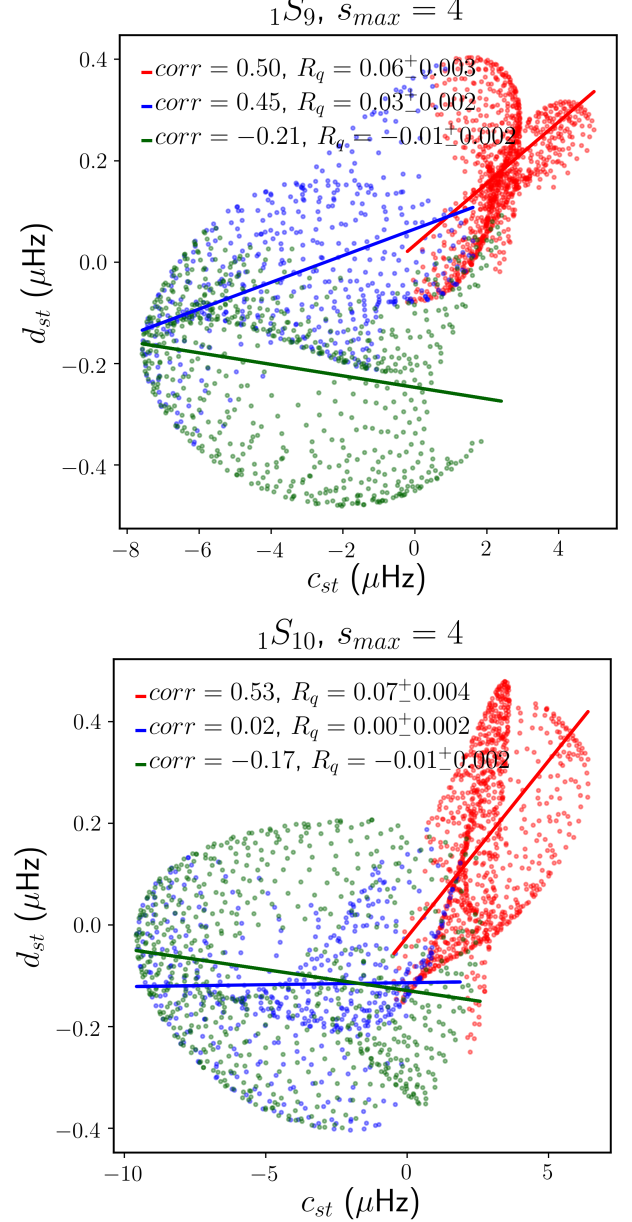

Figure 6.21: $c_{s t}$ versus $d_{s t}$ measurements compared for lower mantle modes ${ }_{1} S_{9},{ }_{1} S_{10}$. (left) Each grey point (1300) represents an equidistant latitude and longitude location in the $c_{s t}$ and $d_{s t}$ splitting function maps. (right) Regional variations for the Pacific LLSVP (blue), the 'ring around the Pacific' (red), and the African LLSVP (green). A simple linear regression is calculated, where corr is the correlation and $R_{q}$ is the slope. See Fig. 5.A.3 to compare the $R_{q}$ in our observations to the one using S20RTS (Ritsema et al., 1999) predictions. 
a) Our $c_{s t}$ measurements

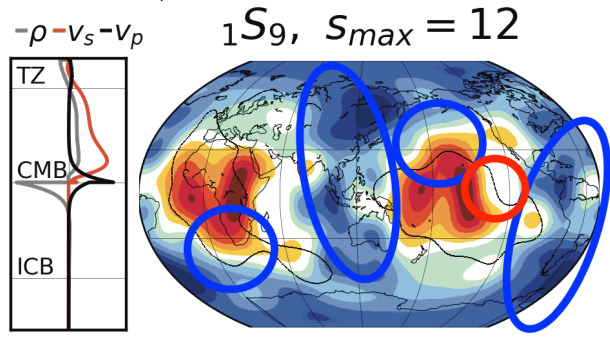

b) S40RTS $c_{s t}$ predictions

c) Our $d_{s t}$ measurements

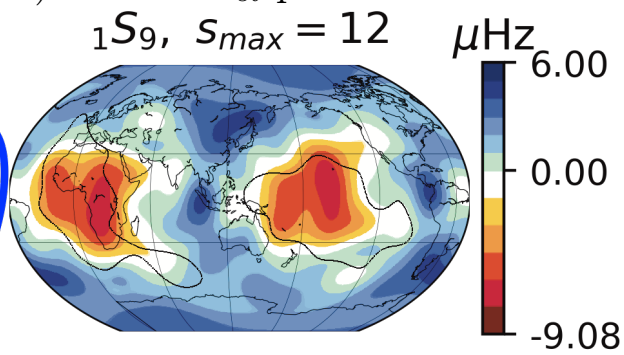

d) QRFSI12 $d_{s t}$ predictions

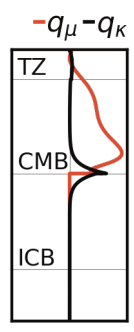

${ }_{1} S_{9}, S_{\max }=4$

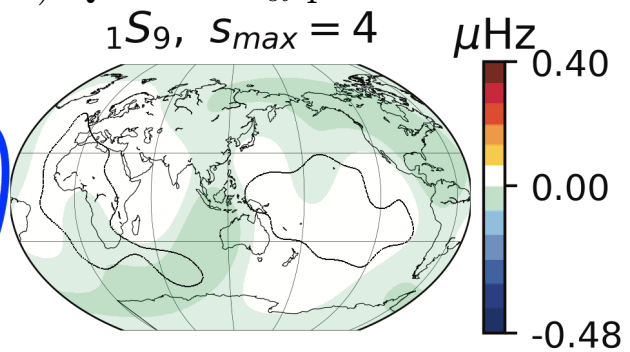

$-9.08$

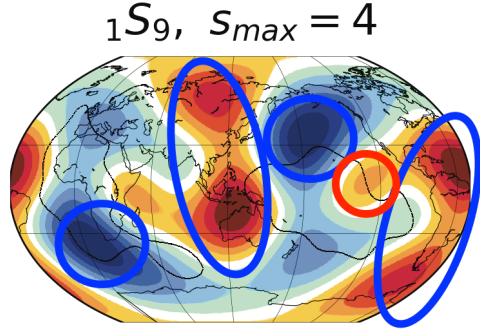

Figure 6.22: Highlighted version of Fig. 6.20, regions showing anti-correlation between elastic and anelastic structure in red circles, correlation in blue circles. See the caption of Fig. 6.17 for explanation.

for upper mantle sensitive modes, with strong attenuation regions in the Pacific and central Indian ocean (Fig. 6.10). Additionally, although Masters et al. (2000a) and Masters et al. (2000b) did not further analyse their anelastic measurements of the mantle, they do report that their anelastic measurement of mode ${ }_{1} S_{5}$ is of significant size, which we also see. Modes ${ }_{1} S_{8},{ }_{1} S_{9}$ and ${ }_{1} S_{10}$, which are mostly sensitive to the lowermost mantle, show instead strong attenuation under Central America, close to the location of the subducting $\operatorname{Cocos}^{2}$ plate, and under Oceania, close to several mid-mantle high velocity anomalies believed to be slabs (e.g. the Sunda ${ }^{2}$ anomaly). In addition, modes ${ }_{1} S_{9}$ and ${ }_{1} S_{10}$ show strong attenuation in North East Asia, close to the Mongol-Kazakh ${ }^{2}$ high velocity anomaly (which is interpreted as a slab), and in the Palmer peninsula, close to the South Orkney ${ }^{2}$ Island slab. In some of these places, there might be a link to water being carried into the lower mantle by subducting slabs leading to strong attenuation, just as Lawrence and Wysession (2006b) inferred under China before.

It is important to point out that both Hwang and Ritsema (2011) and the unpublished whole mantle 3D shear attenuation model VQM3DA (Lawrence and Wysession, 2006c) report the LLSVPs being strongly attenuating (Lawrence and Wysession, 2006b). Romanowicz (1998) also reported seeing strongly attenuating LLSVPs in a preliminary wave-form inversion. These results contradict what we see

${ }^{2}$ Slabs and velocity anomalies documented in the 'Atlas of the underworld' (van der Meer et al., 2018, https://www.atlas-of-the-underworld.org/) 
in the lower mantle, where the strongest attenuation is not in the LLSVPs but in Oceania and central America. The LLSVPs in our observations are not the most weakly attenuation regions either, which are in fact found under the North Pacific and in the Southern Ocean. A reason for this discrepancy might be unaccounted focusing and scattering effects, as well as trade-offs with the scalar moment of earthquakes, as we see in Section 5.3.2. It could also be due to different data coverage, with might be especially problematic for body wave studies which are limited due to uneven source and receiver distribution.

A different study on upper mantle $q_{\mu}$, suggested that the strongly attenuating regions seen in the mantle transition zone might be related to hotspot locations, which are in turn connected to the LLSVPs (Romanowicz and Gung, 2002). However, the model in that study did not extend down to the lowermost mantle and did not include seismic data sensitive to attenuation in the LLSVP regions. Their idea has been further explored in more recent work using a high-resolution $3 \mathrm{D} v_{s}$ mantle model (French and Romanowicz, 2014). The latest of a series of papers by Romanowicz and co-authors indicates that the LLSVPs are not uniform and continuous provinces at the base of the core-mantle boundary, but instead that the LLSVPs are composed of plume bundles, which present themselves as hotspots at the Earth's surface (Davaille and Romanowicz, 2020). Such regional variation within the LLSVPs might be in agreement with our observations of regional variations in attenuation within the LLSVPs.

We would like to further quantify the level of correlation between the elastic and anelastic splitting function observations, just like we did for the upper mantle sensitive modes. This is achieved by plotting our measurements in a similar way as in Fig. 6.18. We again calculate the correlation level by plotting equidistant latitude and longitude points of the $c_{s t}$ and $d_{s t}$ frequency variations in a splitting function map against each other, and then fit the point cloud with a simple linear relationship (see Fig. 6.21). For modes ${ }_{1} S_{9}$ and ${ }_{1} S_{10}$ the correlation between the elastic and anelastic frequency variations is given by 0.41 and 0.40 respectively (Fig. 6.21), confirming the correlation we inferred by comparing our $c_{s t}$ and $d_{s t}$ observations by eye (see Figs. 5.A.2-5.A.3 for comparison). These values are around $40 \%$ larger than the absolute size of the anti-correlation level found in upper mantle sensitive modes (between -0.27 and -0.29 in Fig. 6.18). We also investigate if there are regional variations in the correlation by separating the Earth's surface in areas within and surrounding the LLSVPs. In the 'ring around the Pacific' region we find correlation values of 0.5 and 0.53 , which are higher than the global correlation found for these modes. The Pacific LLSVP shows correlation again, however the level of correlation in this region depends strongly on the mode, with ${ }_{1} S_{9}$ having a much stronger correlations of 0.45 and ${ }_{1} S_{10}$ having almost no correlation of 0.02 . On the other hand, the African LLSVP shows anti-correlation instead of correlation, although with rather small anti-correlation values of -0.21 and -0.17 .

\subsubsection{Comparison with existing 3D $\rho$ models}

In the previous section, we compared our $c_{s t}$ and $d_{s t}$ observations and inferred that our anelastic splitting function observations for the lowermost mantle require 
compositional heterogeneity, and cannot be explained solely by thermal variations in the lower mantle. From mineral physics, we know that compositional variations are expected to have a strong effect on the shear velocity, but not on attenuation. For this reason, it is important to compare our observations to $3 \mathrm{D}$ density models. Models of 3D density variations in the mantle have previously been used to estimate compositional heterogeneity in the lowermost mantle, so we also would like to compare our anelastic splitting function observation with existing density models. All the currently available density models have been obtained using either normal modes or tidal waves and have reported either (i) dense LLSVPs (Ishii and Tromp, 1999; Trampert et al., 2004; Mosca et al., 2012; Moulik and Ekström, 2016; Lau et al., 2017), pointing to a compositional origin and a likely enrichment in iron, (ii) neutrally buoyant or even lighter LLSVPs (Koelemeijer et al., 2017), pointing to a thermal origin or (iii) an inability to meaningfully constrain density using normal modes (Masters et al., 2000c; Romanowicz, 2001; Akbarashrafi et al., 2017; Akbarashrafi, 2020). Fig. 6.23 shows a selection of the currently available 3D density models, including only the models which have been parameterised and obtained independently of shear velocity structure (Ishii and Tromp, 1999; Trampert et al., 2004; Mosca et al., 2012). Two other available lower mantle density studies have used the outline of the LLSVP shape to create regional density models, which have exactly the same shape as in the mantle $v_{s}$ models (Koelemeijer et al., 2017; Lau et al., 2017).

We compare the lowermost mantle 3D $\rho$ models of Ishii and Tromp (1999), Trampert et al. (2004), and Mosca et al. (2012) with our $d_{s t}$ observation of lower mantle sensitive mode ${ }_{1} S_{9}$ (Fig. 6.23). The density models are quite variable, but they do all show some similarity for certain key areas of the LLSVPs. In particular, all three density models show larger density in the north of the Pacific LLSVP, near the Hawaiian region. In the same place, we find weak attenuation (i.e. low $q_{\mu}$ ) in our $d_{s t}$ observation for mode ${ }_{1} S_{9}$. In the same manner, the southern part of the African LLSVP, shows larger density, and weak attenuation in ${ }_{1} S_{9}$. This disagrees with a thermal origin
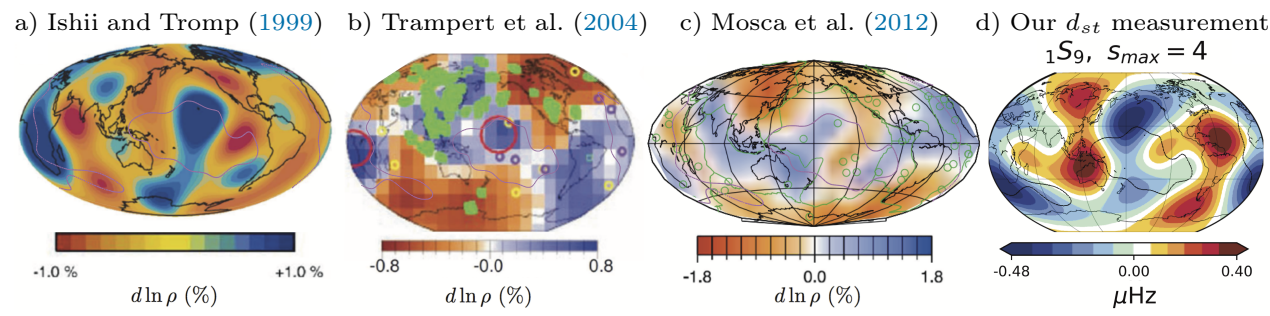

Figure 6.23: Lower mantle 3D density $\rho$ models at $2891 \mathrm{~km}$ depth: (a) Ishii and Tromp (1999) using normal modes; (b) Trampert et al. (2004) using normal modes; (c) Mosca et al. (2012) using body waves, surface waves and normal modes; compared to our (d) $d_{s t}$ measurement of mode ${ }_{1} S_{9}$. Models in (a-c) are plotted in $d \ln \rho(\%)$, where areas in blue show a $\rho$ increase and areas in red show a $\rho$ decrease. The $-0.1 \% v_{s}$ outline of the tomographic model SP12RTS at $2850 \mathrm{~km}$ (Koelemeijer et al., 2016), which contours the LLSVPs, is included for comparison. The red circles in (b) show the LLSVPs regions with the greatest agreement between $\rho$ models in (a-c). 
for the LLSVPs, because these low velocity regions are accompanied by an increase in density and weak attenuation. On the other hand, the eastern part of the Pacific LLSVP, near the Galapagos region, shows a decrease in density, and strong $q_{\mu}$ in ${ }_{1} S_{9}$. This would agree with a thermal origin for the eastern part of the Pacific LLSVP. So, we find that different parts of the LLSVPs may have a different origin, with some parts potentially being thermal and other parts requiring compositional heterogeneity. This would suggest that the LLSVPs do not necessarily have the same uniform compositional or thermal origin and require regional variations within the LLSVPs.

\subsubsection{Mineralogical interpretation}

There is limited information on anelastic properties of lower mantle minerals. Torsional forced-oscillation experiments need to be performed at room pressure, so experiments on lower mantle minerals at lower mantle pressures are not possible. This means that our knowledge comes from experiments on minerals at room pressure which are used as analogues for lower mantle minerals. In addition, our knowledge relies on the assumption that we can extrapolate their results to lower mantle pressure and temperature conditions. Experimental deformation studies on $\mathrm{MgO}$ (Webb and Jackson, 2003; Barnhoorn et al., 2016) and lower mantle analogue titanate perovskites (Webb et al., 1999) have found that they behave in a very similar fashion as olivine, and that increasing temperature also leads to a lowering of the shear modulus $\mu$ and an increase in shear attenuation $q_{\mu}$. Thus, if temperature would be dominant in the lower, then we would expect to find an anti-correlation between the elastic and anelastic splitting functions, like in the upper mantle, which is not what we observe. We find regional variations in the anelastic splitting functions which show much more variation than in the elastic splitting functions which are dominated by the degree-two structure of the LLSVPs. The only region where we find anti-correlation between the elastic and anelastic splitting functions is in the eastern part of the Pacific LLSVP near the Galapagos region, which would suggest a thermal origin.

We need another explanation to interpret the strong attenuation that we find in the high velocity regions surrounding the LLSVPs. Experimental data show that partial melting leads to a significant increase in attenuation in olivine (Jackson et al., 2004; McCarthy and Takei, 2011) and the same may happen when perovskite partially melts. Partial melting is most likely present in small patches where Ultra Low Velocity Zones (ULVZs) are found, which are characterised by extremely strong shear wave velocity reduction by up-to $25 \%$ (Rost et al., 2005). ULVZs may be too small to be observed with normal modes (they are only a few tens of kilometers thick), but we did attempt anyway to compare regions where ULVZs have been observed using body waves studies with our anelastic normal mode splitting function observations for lower mantle sensitive modes ${ }_{1} S_{8},{ }_{1} S_{9}$ and ${ }_{1} S_{10}$ (Fig. 6.24a). As we already expected, we do not find a clear link between the locations of ULVZ detection and strong attenuation in our anelastic splitting function measurements. Furthermore, the presence of partial melting would most likely lower the velocity in combination with strong attenuation, which is not what we observe (our strongest attenuation is in regions with increased shear wave velocity). 
a)
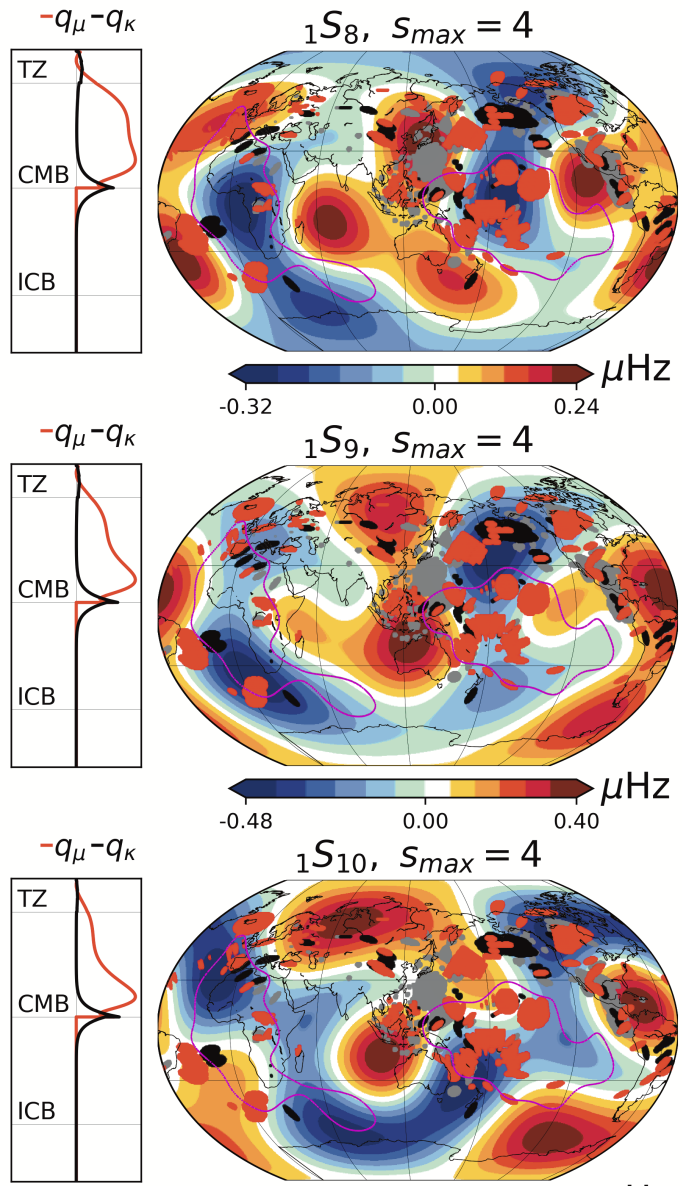

ULVZ studies

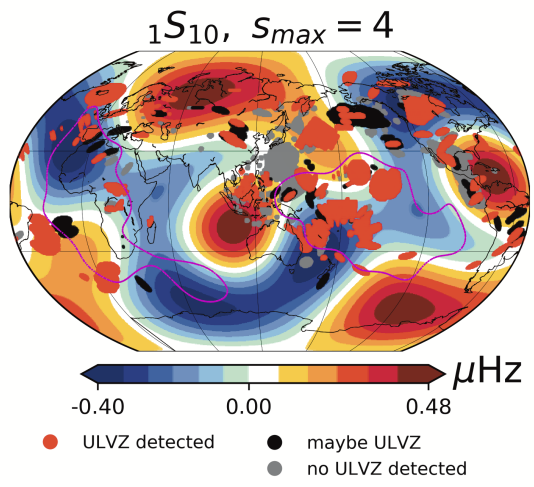

b) Post-perovskite studies

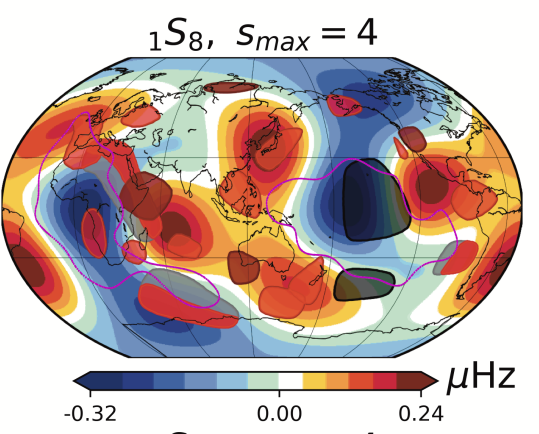

${ }_{1} S_{9}, S_{\max }=4$
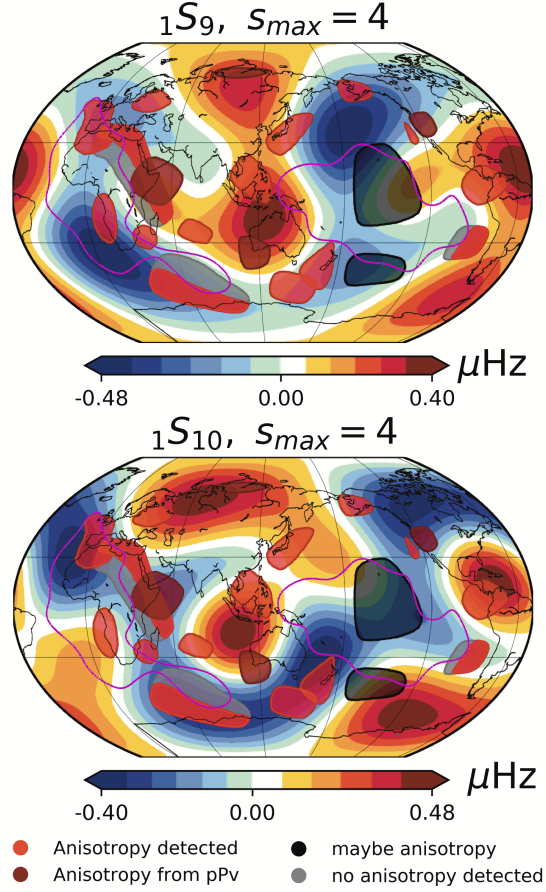

Figure 6.24: Anelastic measurement of modes ${ }_{1} S_{8},{ }_{1} S_{9},{ }_{1} S_{10}$ plotted together with their respective sensitivity kernels for $q_{\mu}$ (red line) and $q_{\kappa}$ (black line). Measurements are compared to (a) suspected Ultra Low Velocity Zones (ULVZs) (Yu and Garnero, 2018); and (b) D" regions where seismic anisotropy has been studied and linked to post-perovskite (pPv) presence (Nowacki et al., 2011; Creasy et al., 2019).

Another alternative explanation could be the presence of water. In olivine, the presence of water leads to increased attenuation and has a much smaller effect on velocity (Karato, 2003; Shito et al., 2006; Aizawa et al., 2008). Water has indeed been used in interpreting previous upper mantle attenuation studies (e.g. Dalton 
et al., 2008; Dalton et al., 2009) for regions with strong attenuation in combination fast velocity, or no significant velocity anomaly. However, water is unlikely to be found in the lower mantle and it is not expected to survive at lower mantle pressure and temperature, so we do not think it is likely that the presence of water is able to explain the strong attenuation found in the regions surrounding the LLSVPs.

We propose that the presence of post-perovskite can explain for our observations. In the deeper parts of the lower mantle, perovskite may deform to post-perovskite (Murakami et al., 2004; Oganov and Ono, 2004; Tsuchiya et al., 2004). The phase transition from perovskite to post-perovskite has been used to explain the D" discontinuity at approximately $250 \mathrm{~km}$ above the core mantle boundary and also the presence of anisotropy in the lowermost mantle (Lay and Garnero, 2011; Romanowicz and Wenk, 2017). From ab-initio molecular simulations we know that post-perovskite is potentially strongly attenuating mineral (Goryaeva et al., 2016), while experiments show it is also potentially fine-grained (Yoshino and Yamazaki, 2007). Post-perovskite is most likely present in the colder fast velocity areas and it might be strongly attenuating, which is precisely what we observe in the correlation between our elastic and anelastic splitting functions for lower mantle sensitive modes. For these reasons, we interpret the high attenuation in the 'ring around the Pacific' as evidence for the presence of post-perovskite. Given that our observations of anelasticity have a very low structural degree, it is difficult to compare them directly to body waves studies of the D" region and properly match both types of observations (Fig. 6.24b). Nevertheless, we do find that our interpretation agrees with strong anisotropy found in the same regions, which has also been attributed to the presence of post-perovskite in the ring around the Pacific surrounding the LLSVPs (Romanowicz and Wenk, 2017; Creasy et al., 2019). Thus, observations of regional variations in attenuation for the lower mantle may provide a new additional tool to constrain the presence of post-perovskite in the lowermost mantle.

Alternatively, our attenuation observations can also be explained by simultaneous grain-size and temperature variations. The complex relationship between grain-size and temperature is affected by the level of stress acting on different regions of the mantle, as well as by a decay in the grain-growth rate with depth along the mantle (Dannberg et al., 2017). A recent study (Dannberg et al., 2017) using geodynamical and laboratory-based models showed that high stresses within the cold slabs are expected to lead to small grain-sizes, resulting in stronger seismic attenuation. Stable hot LLSVPs, on the other hand, would potentially be large grain-size provinces with weaker attenuation. This potentially explains our lower mantle observations, where stronger attenuation in combination with higher velocity is present in the 'ring around the Pacific', as being due to a simultaneous decrease in grain-size and temperature under high stresses. The weaker attenuation together with the lower velocities that we find in the LLSVPs regions would then be explained by a simultaneous increase in grain-size and temperature.

We find the weakest attenuation in two regions bordering the LLSVPs, around Hawaii and southern Africa. In the same region, previous studies have inferred larger density than the surrounding mantle (Ishii and Tromp, 1999; Trampert et al., 2004; Mosca et al., 2012). This larger density would indicate a compositional heterogeneity, 


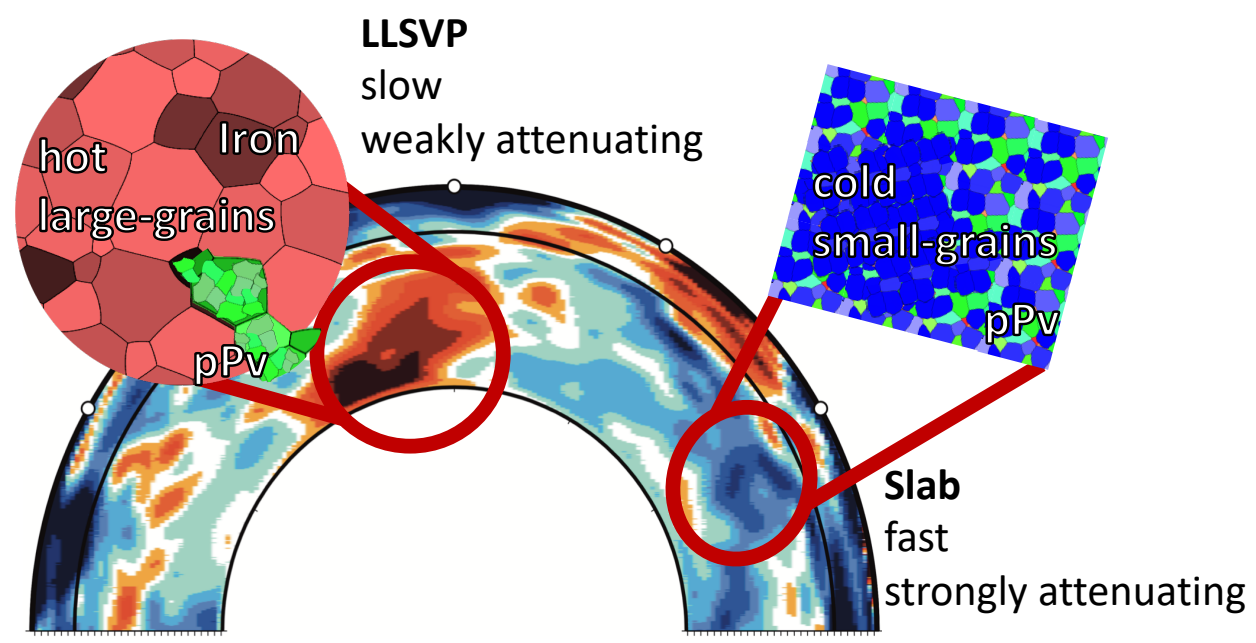

Figure 6.25: Schematic representation of our mineral physics interpretation of our lower mantle observations.

most likely enrichment in iron, and not an increase in temperature, which is in agreement with our weak attenuation found in the same places. This potential iron enrichment is also affected by the oxidation state of iron in the lower mantle. Creasy et al., 2020 conducted diamond anvil experiments relating the iron oxidation state in a bridgmanite sample (i.e. magnesium-rich silicate perovskite). They find that, at lower mantle conditions, the reduced sample (less $\mathrm{Fe}^{3+}$ ) increases in density by $\sim 1.5 \%$ and decreases in velocity by $\sim 2 \%$, which agrees with seismological observations. This means that not only iron content, but also its oxidation or redox state, matters when interpreting lower mantle compositional variations.

In the central parts of the LLSVPs we find average attenuation, which means stronger attenuation than the weakest attenuating regions near Hawaii and southern Africa. How to interpret this average attenuation depends on the magnitude of the attenuation increase due to either the presence of post-perovskite or due to the higher temperature and grain-size of $\mathrm{MgO}$ and perovskite within the LLSVPs. Post-perovskite presence may increase the expected attenuation (Goryaeva et al., 2016) of the LLSVPs and lead to non-dominant degree-two structure and average attenuation at low structural degrees, as well as overwhelm the effect of temperature and grain-size on perovskite in the central LLSVPs. In this case, the observed attenuation in the central parts of the LLSVPs could still be partly due to an increase in temperature and grain-size, and not a change in composition. 


\subsection{Conclusion}

We measure elastic and anelastic structure simultaneously for modes sensitive to the upper and lower mantle. This allows us to measure intrinsic attenuation and automatically include potential trade-offs with elastic effects, such as focussing and scattering. In the upper mantle, we find that low velocity regions are correlated with strongly attenuating regions, which agrees with previous studies using surface and body waves. Comparing these upper mantle observations with experimental results for olivine indicates that our observations are dominated by lateral temperature variations, which again is in agreement with previous studies.

In the lower mantle (Fig. 6.25), we find that the strongest attenuation is located in two high velocity regions centered around Oceania and central America, and not in the LLSVPs. The strong attenuation found in the high velocity regions surrounding the LLSVPs is opposite of what we see in the upper mantle. This can potentially be explained by small grain-sizes in combination with cold temperatures, agreeing with (Dannberg et al., 2017), and/or the presence of post-perovskite, which recently has been reported to be strongly attenuating (Goryaeva et al., 2016) and fine-grained (Yoshino and Yamazaki, 2007). The weakest attenuation is found in two low velocity regions, one in the northern part of the Pacific LLSVP near Hawaii and another in the southern part of the African LLSVP. This suggests that these two regions might be dominated by compositional variations instead of temperature, potentially due to iron enrichment in agreement with larger density found in the same places (e.g. Ishii and Tromp, 1999). In the central part of the LLSVPs we find average attenuation, which could be due to higher temperatures and large grain-sizes in combination with post-perovskite, which might overwhelm the temperature/grain-size effect of perovskite in the LLSVPs. 


\section{A Appendix: $c_{s t}+d_{s t}$ damping curves}
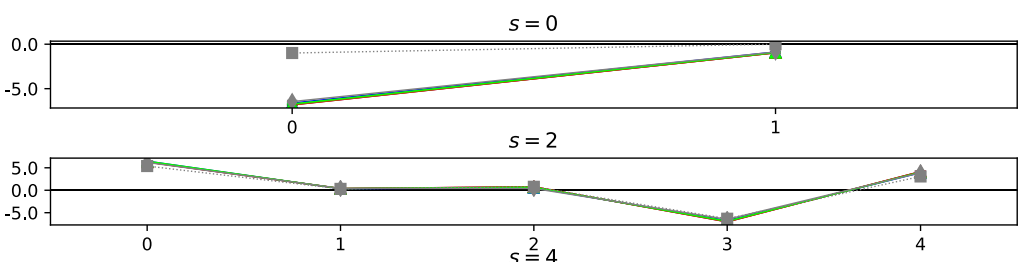

mzero

$\rightarrow \mathrm{d}=0.0001, \mathrm{it}=10$

$-d=0.001$, it $=10$

$\neg d=0.01, i t=10$

$\rightarrow \mathrm{d}=0.1$, it $=10$

1... S4ORTS
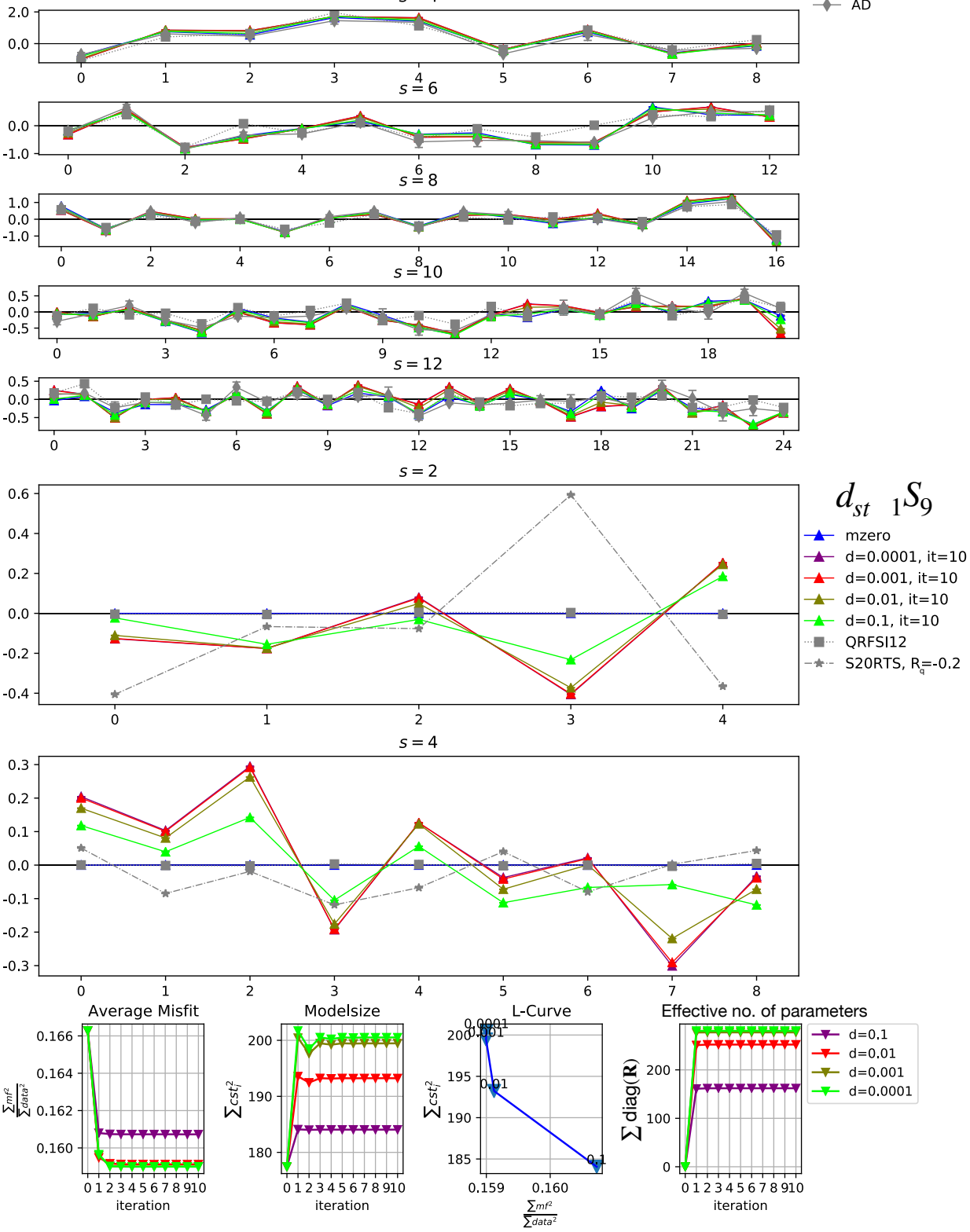

Figure 6.A.1: Damping curves of the inversion of mode ${ }_{1} S_{9}$. Also shown are the average misfit, model size and effective eigenvalue per iteration and damping, together with the L-curve of the inversion. The chosen damping was 0.01 . 

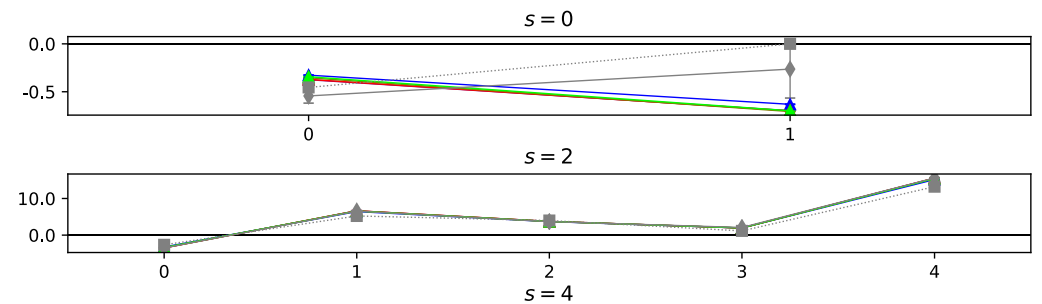

$\rightarrow$ mzero

- $\mathrm{d}=0.0001, \mathrm{it}=10$

$-\mathrm{d}=0.001$, it $=10$

$\rightarrow \mathrm{d}=0.01$, it $=10$

$-\mathrm{d}=0.1$, $\mathrm{it}=10$

-.... S4ORTS

$-A D$
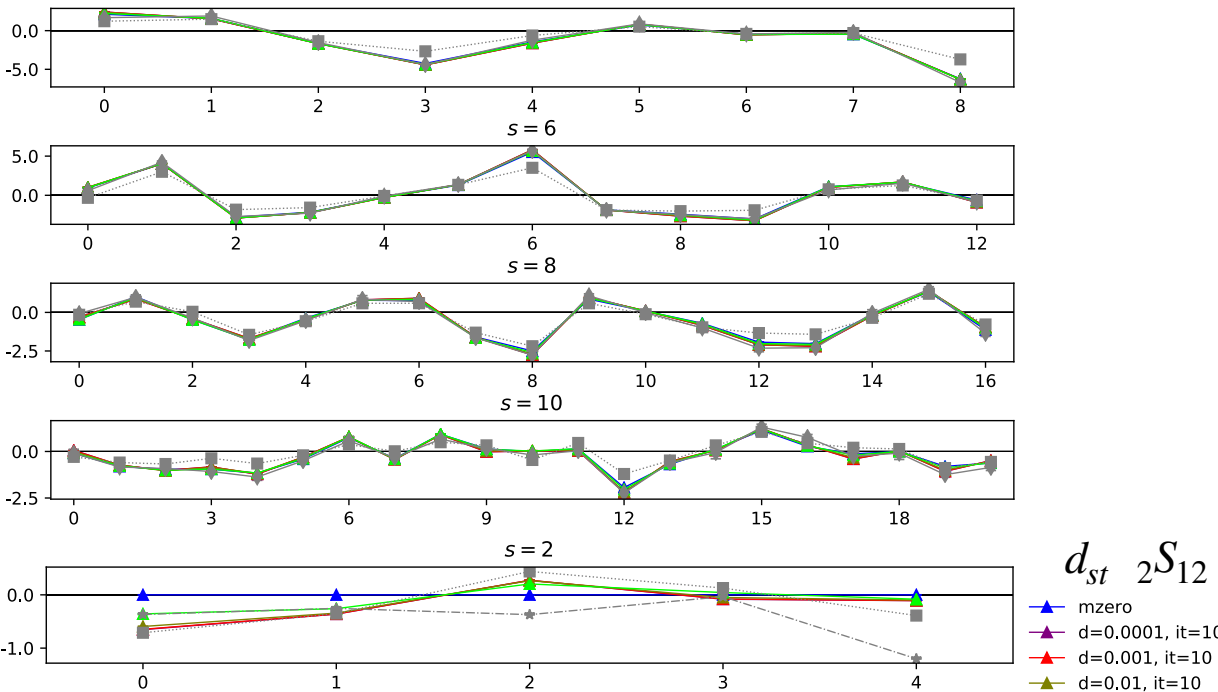

- mzero

$\neg \mathrm{d}=0.0001, \mathrm{it}=10$

$-\mathrm{d}=0.001$, it $=10$

$\rightarrow \mathrm{d}=0.01$, it $=10$

$\rightarrow-d=0.1$, it $=10$

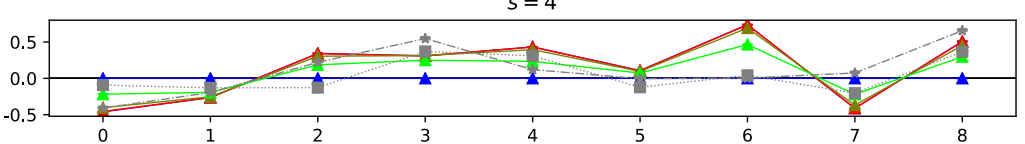

QRFSII2

*- S2ORTS, $R_{\overline{4}}=-0.2$

$s=6$
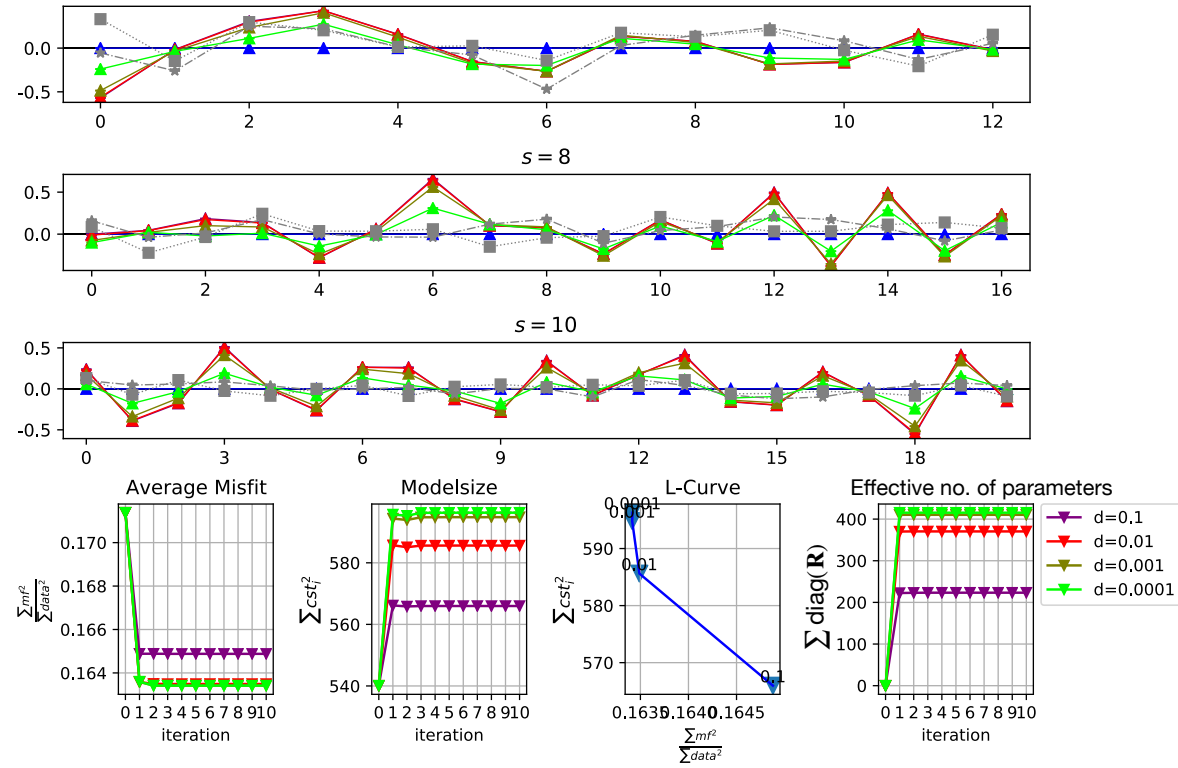

Figure 6.A.2: Damping curves of the inversion of mode ${ }_{2} S_{12}$. The chosen damping was 0.01 . See the caption of Fig. 6.A.1 for explanation. 


\section{B Appendix: $d_{s t}$ observations at larger structural degrees}

Our $c_{s t}$ measurements
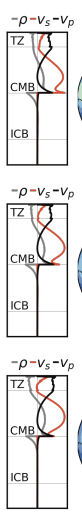

${ }_{0} S_{6}, S_{\max }=4$

$S_{7}, S_{\max }=4$

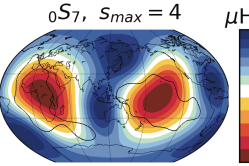

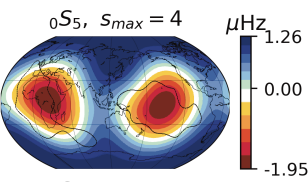
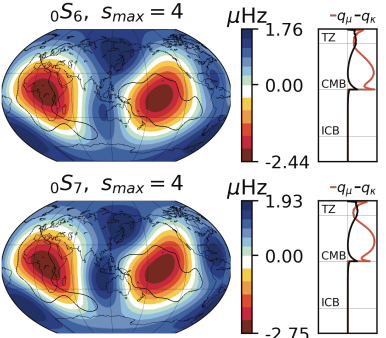

Our $d_{s t}$ measurements

${ }_{0} S_{5}, S_{\max }=4$

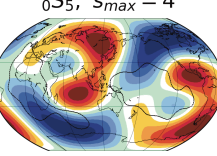

${ }_{0} S_{6}, S_{\max }=4$

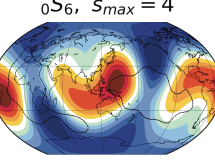

${ }_{0} S_{7}, S_{\max }=4$

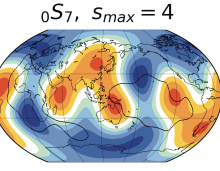

S20RTS $d_{s t}$ predictions QRFSI12 $d_{s t}$ predictions $\delta q_{\mu}=-0.2 \delta v_{s} / v_{s} \quad$ Upper mantle $3 \mathrm{D} q_{\mu}$

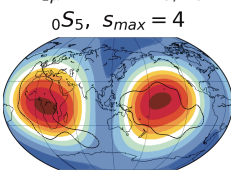
${ }_{0} S_{5}, S_{\max }=4$

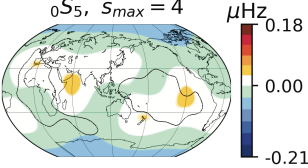

${ }_{0} S_{6}, S_{\max }=4$

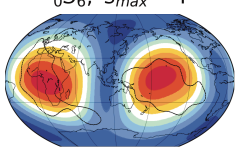

${ }_{0} S_{6}, S_{\text {max }}=4 \quad \mu \mathrm{Hz}{ }_{0.29}$

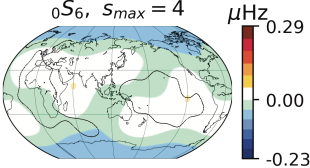

${ }_{0} S_{7}, S_{\max }=4$
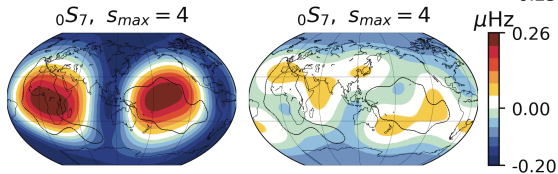

Figure 6.B.1: Splitting function $c_{s t}\left(1^{\text {st }}\right.$ column $)$ and $d_{s t}\left(2^{\text {nd }}\right.$ column $)$ measurements of fundamental modes ${ }_{0} S_{l}$, plotted together with their respective sensitivity kernels for $v_{s}$ and $q_{\mu}$ (red lines), $v_{p}$ and $q_{\kappa}$ (black lines) and $\rho$ (grey line). All kernels are plotted using PREM for degree-zero. Measurements are compared to anelastic splitting function predictions up to degree 4 for our 3D synthetic anelastic model $\left(3^{\text {rd }}\right.$ column) and 3D anelastic upper mantle model QRFSI12 (up to $400 \mathrm{~km}$ ) (4 ${ }^{\text {th }}$ column, Dalton et al., 2008). The $-0.1 \% v_{s}$ outline of the tomographic model SP12RTS at $2850 \mathrm{~km}$ (Koelemeijer et al., 2016), which contours the LLSVPs, is included for comparison.

Our $c_{s t}$ measurements

Our $d_{s t}$ measurements
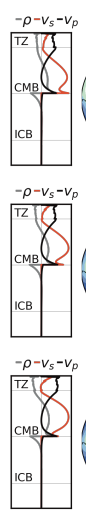
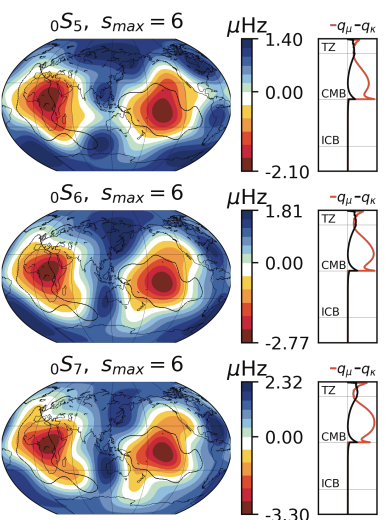
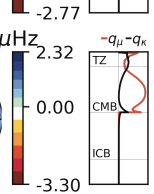
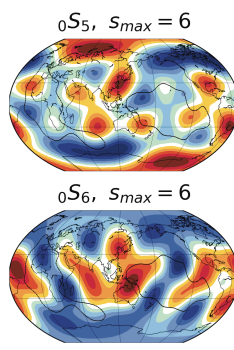

${ }_{0} S_{7}, S_{\max }=6$

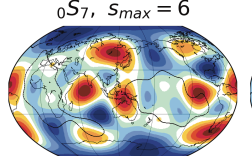

S20RTS $d_{s t}$ predictions QRFSI12 $d_{\text {st }}$ predictions $\delta q_{\mu}=-0.2 \delta v_{s} / v_{s} \quad$ Upper mantle 3D $q_{\mu}$
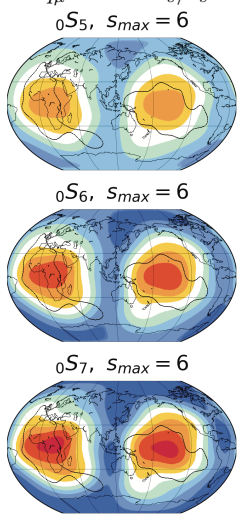
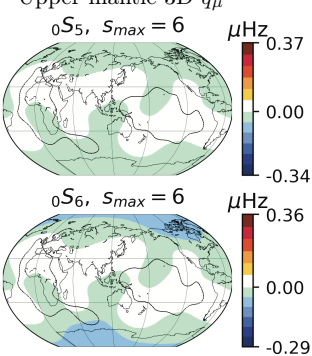

${ }_{0} S_{7}, S_{\text {max }}=6 \quad \mu \mathrm{Hz}$

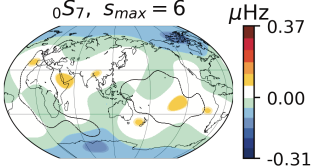

Figure 6.B.2: Splitting function measurements of fundamental modes ${ }_{1} S_{l}$ compared to mantle model predictions for $s_{\max }=6$. See the caption of Fig. 6.B.1 for explanation. 
Our $c_{s t}$ measurements
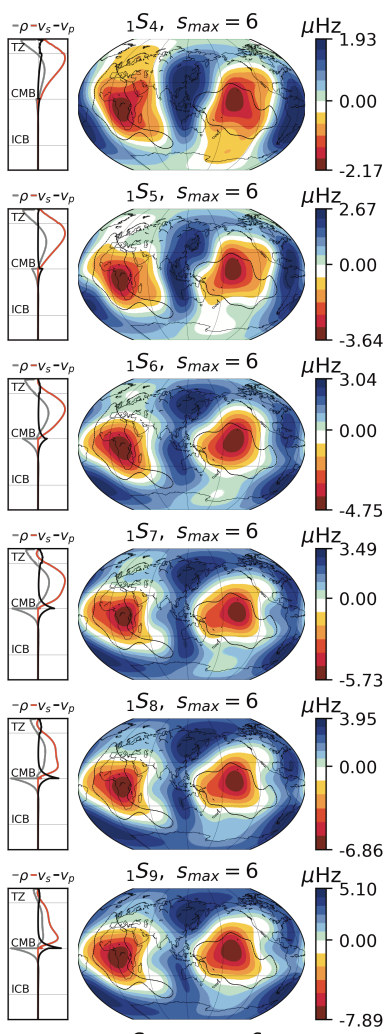

$\left.\right|_{\text {ICB }} ^{2}$
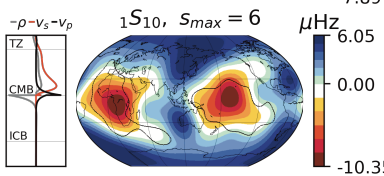

Our $d_{s t}$ measurements

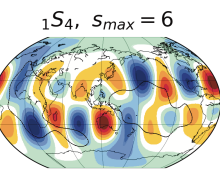

${ }_{1} S_{5}, S_{\max }=6$
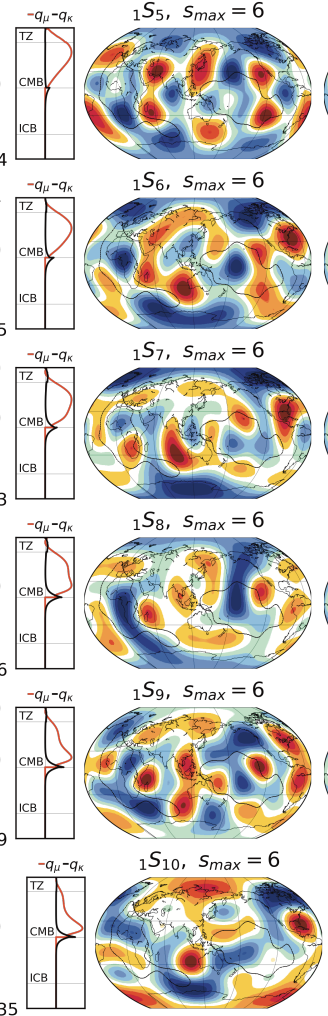

S20RTS $d_{s t}$ predictions QRFSI12 $d_{s t}$ predictions $\delta q_{\mu}=-0.2 \delta v_{s} / v_{s} \quad$ Upper mantle $3 \mathrm{D} q_{\mu}$
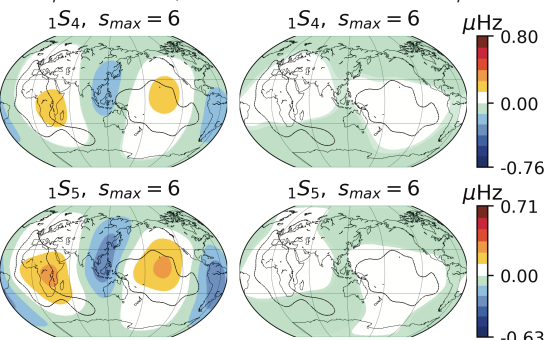

${ }_{1} S_{5}, s_{\max }=6 \quad \mu \mathrm{Hz}$

${ }_{1} S_{6}, S_{\max }=6$
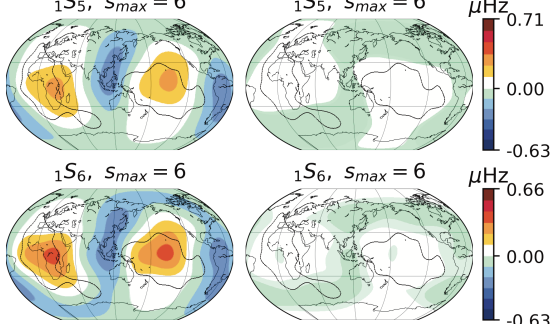

${ }_{1} S_{6}, S_{\max }=6 \quad \mu \mathrm{Hz}$
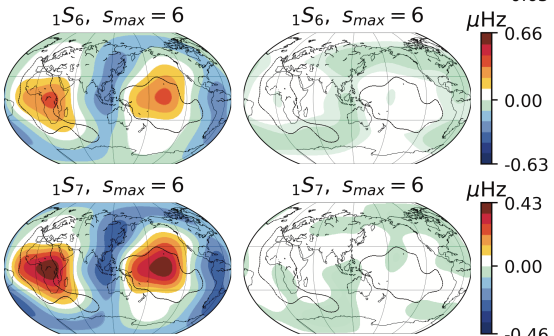

${ }_{1} S_{7}, s_{\max }=6 \quad \mu \mathrm{Hz}$

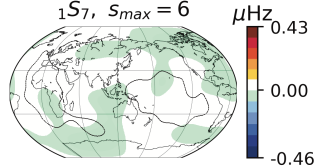

${ }_{1} S_{8}, S_{\max }=6$

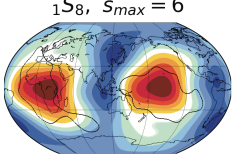

${ }_{1} S_{8}, S_{\max }=6 \quad \mu \mathrm{Hz}$

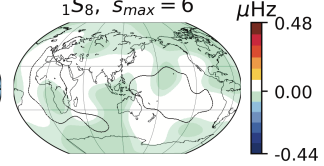

${ }_{1} S_{9}, S_{\max }=6$

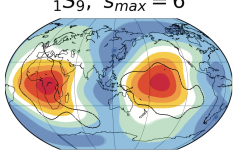

${ }_{1} S_{9}, S_{\max }=6 \quad \mu \mathrm{Hz}$

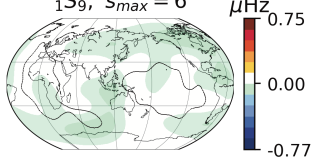

${ }_{1} S_{10}, S_{\max }=6$

${ }_{1} S_{10}, S_{\max }=6 \quad \mu \mathrm{Hz}$
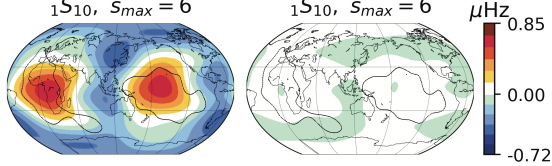

Figure 6.B.3: Splitting function measurements of first overtone modes ${ }_{1} S_{l}$ compared to mantle model predictions for $s_{\max }=6$. See the caption of Fig. 6.B.1 for explanation. 

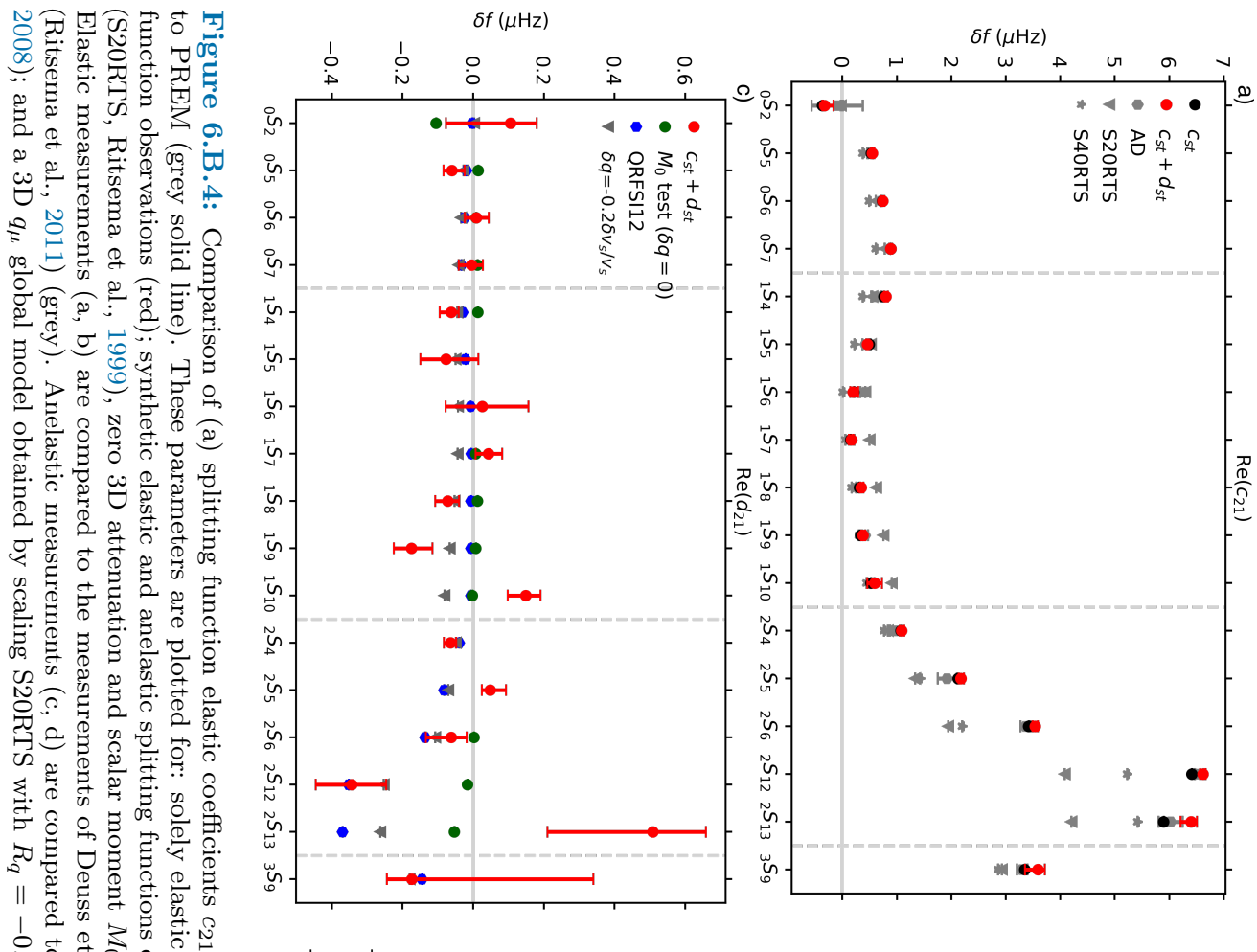

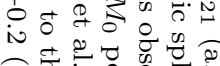
ชิ

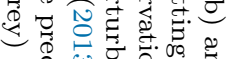

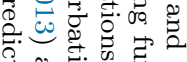
茟 की

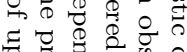
0
0 记

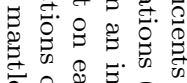

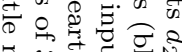

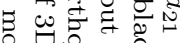

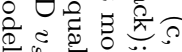
०)

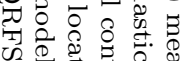

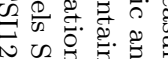
N

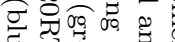
ठ एँ

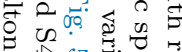

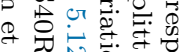
ข

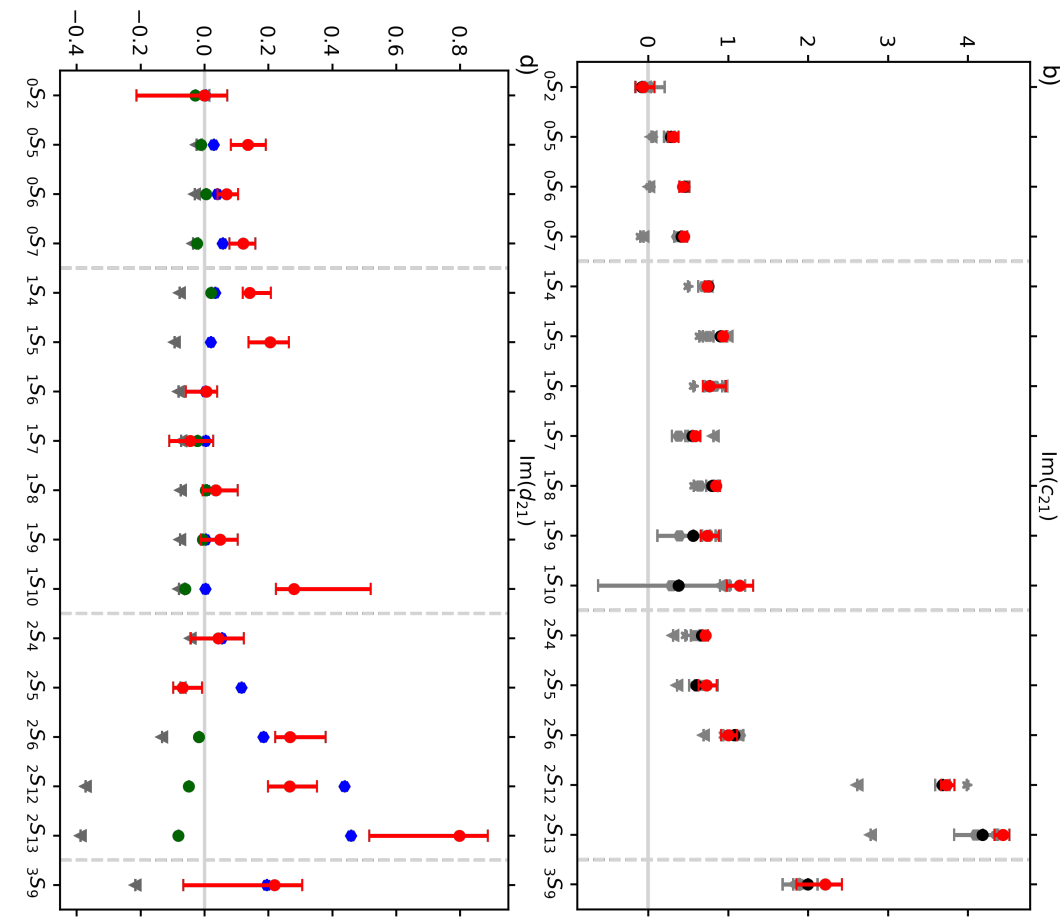




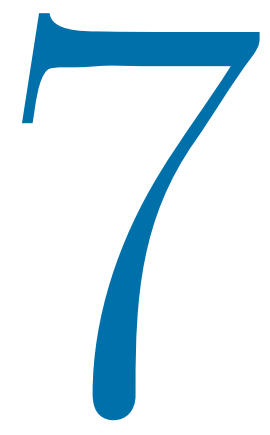

\section{Synthesis}

The main focus of this thesis is to study $1 \mathrm{D}$ variations in inner core attenuation and $3 \mathrm{D}$ variations in mantle attenuation using whole Earth oscillations, or normal modes. We are using splitting functions, which are depth weighted averages of how a particular normal mode 'sees' the Earth, and are a first step towards seismic tomography. Although our chosen method does not enable us to make the same inferences as a seismic tomography model of attenuation variations, measuring splitting functions does allow us to investigate the interior of our planet and draw significant conclusions before diving into constructing a tomographic model with our splitting functions. In this synthesis, we will first summarise the main conclusions of the thesis, and later discuss the challenges we faced and the future work we propose to further develop our findings.

First, in Chapter 3 we study the role of $1 \mathrm{D}$ attenuation cross-coupling on the controversial inner core sensitive normal mode pair ${ }_{10} S_{2^{-} 11} S_{2}$. Early studies of modes sensitive to inner core shear wave velocity and attenuation, such as ${ }_{10} S_{2}$ and ${ }_{11} S_{2}$, showed evidence of a weakly attenuating inner core. With time, as more inner core studies of compressional velocity sensitive modes were performed, it was shown that other modes prefer a strongly attenuating inner core instead. Here, we show that despite previous inconsistencies, by allowing modes ${ }_{10} S_{2}$ and ${ }_{11} S_{2}$ to cross-couple through 1D structure, our measurements of these modes agree with a strongly attenuating inner core. We propose, that the reason for the previous disagreement is the strong exchange of energy between these modes, which changes their mode characteristics as a consequence of a $0.5 \%$ perturbation to either inner core shear velocity or radius.

Next, in Chapter 4 we study radial modes, which mostly depend on compressional energy, in order to constrain bulk attenuation $q_{\kappa}$. Previous 1D attenuation models vary significantly for $q_{\kappa}$, ranging in depth, thickness and amplitude of the layer with finite $q_{\kappa}$ by several orders in magnitude. Radial modes were last measured more than a decade ago using only one or two earthquakes. Here, we measure radial modes using 16 of the strongest and deepest earthquakes of the last two decades. Our measured $Q$ value of ${ }_{0} S_{0}$ requires less finite $q_{\kappa}$ somewhere in the Earth than previous measurements. By comparing our $Q$ observations to synthetic $1 \mathrm{D} q_{\kappa}$ models, we select the upper mantle as the preferred location for finite bulk 
attenuation to best fit our measurements. We found that radial modes cross-couple strongly to their nearest spheroidal mode due to ellipticity and inner core cylindrical anisotropy. Including cross-coupling in our measurements results in a systematic shift of the centre frequencies of the radial modes towards the Preliminary Reference Earth Model (PREM) frequencies. This smaller frequency difference with PREM is consistent with lower values of radial anisotropy in the inner core than previously inferred. The centre frequencies of radial modes measured in self-coupling will result in an overestimation of radial inner core anisotropy.

Chapters 3 and 4 also provide new measurements of splitting functions for inner core sensitive modes, both in self-coupling and in cross-coupling, some of which have never been measured before. Several of these modes are sensitive to shear wave velocity in the innermost core, for which not many measurements have been available so far. Our measurements provide new constraints on cylindrical inner core anisotropy in the innermost inner core, and can be easily included when making future models of inner core anisotropy.

Then we shift our attention from the inner core to studying $3 \mathrm{D}$ variations in mantle attenuation. In Chapter 5, we evaluate the feasibility of constraining 3D variations in mantle attenuation using our method of measuring splitting functions from normal mode spectra. We performed forward modelling and tests on synthetic data and show that our method is sufficiently robust to measure anelastic splitting functions (dependent on attenuation) in addition to the commonly measured elastic splitting functions (dependent on velocity and density) for mantle sensitive modes. Furthermore, we find that the effect of earthquake magnitude errors might be a problem only when the magnitude of lower mantle anelastic splitting functions is less than $50 \%$ of the scaled amplitude signal of expected upper mantle attenuation $\left(\delta q_{\mu}<|0.1| \delta v_{s} / v_{s}\right)$. Additionally, using the expected strength of upper mantle attenuation, we found that anelastic splitting functions can be measured confidently for the $1^{\text {st }}$ and $2^{\text {nd }}$ overtones.

Finally, in Chapter 6 we measure anelastic splitting functions for upper and lower mantle sensitive modes using real earthquake data and interpret these in terms of $3 \mathrm{D}$ variations in mantle attenuation. Our observations for upper mantle sensitive modes agree with previous studies, and show that low velocity regions are correlated with strongly attenuating regions, which indicates a thermal origin for the seismic anomalies. In the lower mantle, on the other hand, we do not find the strongest attenuation in the Large Low Shear Velocity Provinces (LLSVPs) under African and the Pacific. Instead, the strongest attenuation is found in the high velocity regions surrounding the LLSVPs, which we interpret as potentially being due to small grain-sizes in combination with cold temperatures, and/or the presence of post-perovskite, which may be strongly attenuating. The weakest attenuation is found in two low velocity regions, one in the northern part of the Pacific LLSVP near Hawaii and another in the southern part of the African LLSVP. This suggests that these two regions might be dominated by compositional variations instead of temperature, potentially due to iron enrichment in agreement with larger density found in the same places. Within the LLSVPs we observe average attenuation, which could still be due to higher temperature and large grain-sizes in combination 
with post-perovskite, which might overwhelm the temperature/grain-size effect of perovskite within the LLSVPs.

Overall, we have shown in this thesis that normal modes are able to constrain both $1 \mathrm{D}$ and $3 \mathrm{D}$ variations in attenuation in the mantle and inner core. Collecting sufficient earthquake data is key to obtain robust observations, and will help overcome other challenges that might arise from uncertainties and noise in our data and inaccuracies in the earthquake moment magnitudes. We expect that in the future, our anelastic normal mode splitting function observations and corresponding attenuation values will be able to add new constraints to the next generation of $3 \mathrm{D}$ tomographic attenuation models. Additionally, we envision our findings being further interpreted in collaborations with other disciplines, such as mineral physical experiments, molecular simulations and geodynamical modelling, in order to validate the origin, we proposed for our attenuation observations.

\subsection{General challenges in this thesis}

Constraining attenuation in the Earth using normal modes is not straightforward, and we faced several challenges in making the $Q$ and anelastic splitting function measurements. Some of these are discussed in the relevant chapters, and here we highlight the following:

- The iterative spectral fitting method requires knowledge of the moment tensor solution of each earthquake in our catalogue. These moment tensor solutions and especially the moment magnitudes have their own uncertainties, which may be under-predicted and in turn may alter our anelastic measurements. We estimated an upper-bound for the influence of moment magnitude uncertainties, which is normal mode dependent, and we only interpret anelastic splitting function measurements for which the potential moment magnitude effect is small enough.

- Given that the least-squares inversion scheme does not provide a probability distribution for each of our inverted parameters, we calculate uncertainties by removing whole events at the time. In some cases, this method may under-predict the real errors in our measured parameters, which is especially important when measuring anelastic splitting functions, that have much smaller amplitudes than the elastic splitting functions. We solved this by running many additional tests for our inversion method, including the influence of the starting model and inaccuracies in the earthquake moment magnitude solutions.

- So far, we have only measured anelastic splitting functions, and we did not yet use these to make a 3D attenuation model of the mantle. Therefore, we cannot specify a particular depth range for our attenuation measurements and only roughly divide them into upper or lower mantle sensitive, because of the depth averaging nature of the sensitivity kernels of the splitting functions. We also do not distinguish between $Q_{\mu}$ and $Q_{\kappa}$ observed by individual modes because their $Q$ depends on both. Making a $1 \mathrm{D}$ and 3D model of mantle attenuation will be the focus of our immediate future research. 
- The amplitude of the anelastic splitting functions is an order of magnitude smaller than the elastic splitting functions, which creates problems when measuring the anelastic and elastic splitting functions in a joint inversion. We solved this by first measuring only the elastic splitting functions, and then using these as a starting model in the joint inversion.

- Measuring the anelastic splitting functions of the key modes ${ }_{0} S_{2},{ }_{10} S_{2}$ and ${ }_{11} S_{2}$ was not possible with the currently available data. Especially ${ }_{0} S_{2}$ would be able to provide important constraints on $3 \mathrm{D}$ variations in lower mantle attenuation, but because of the presence of too much noise at the very low frequency of this mode, only a handful of spectra are available.

- Finally, we experienced time limitations due to

(i) The use of an un-parallelised legacy code, which we overcame by creating wrappers around the code to distribute the calculation over different processors in our large computer cluster for each earthquake in the dataset, and

(ii) The processing of new earthquakes and selection of new spectral segments, which was very time-consuming and was especially needed to measure longperiod radial modes and their cross-coupling to their nearest inner core sensitive modes. In addition, we also extended the previous earthquake catalogue (up-to 2010) to include all recent large events (up-to 2018) for all other measured modes.

\subsection{Future research directions}

There are a number of interesting research topics that directly follow from this thesis, such as constructing new 1D and 3D models of attenuation. Due to time limitations they were beyond the scope of this thesis, but they are important, and timely, to be tackled in the future. Here we highlight some of these research topics in more detail:

- Given that the most recent 1D $Q$ models that were made using normal modes are Resovsky et al. (2005) and de Wit et al. (2014), we would like to construct a new 1 D attenuation model for $Q_{\mu}$ and $Q_{\kappa}$ using both our new mode $Q$ measurements, as well as other normal mode, body wave and surface wave data. This will allow us to compare our model to previous 1D $Q$ studies, and make interpretations in terms of the thermal and mineralogical structure of the mantle and inner core.

- Following on from our 1D normal mode model, the next step is to construct a new model of the frequency dependence of attenuation using our new $Q$ measurements together with other data types, and compare them with recent frequency models built using either surface waves together with normal modes (Lekić et al., 2009), or body tides together with normal modes (Lau and Faul, 2019).

- We have found weak attenuation in the LLSVPs and strong attenuation in the surrounding regions. It will be interesting to perform a body wave study of lower mantle attenuation using shear waves, focused on the lower mantle regions showing especially strong or weak attenuation in our normal mode anelastic measurements. By comparing the results of both data types, we may understand the 
reason for the discrepancy between our normal mode anelastic observations and the attenuation variations found in previous lower mantle body waves studies.

- So far, we have only measured anelastic splitting functions for a small number of modes. In order to expand our anelastic measurements catalogue, we would like to perform additional anelastic splitting function measurements, including modes with larger $n$ and $l$. Additionally, we should analyse whether or not the use of derivative damping (i.e. varying damping according the angular order of the splitting function coefficients) would have an effect on our anelastic splitting function observations.

- One of the most obvious next steps, would be to construct a new global tomographic model of mantle 3D attenuation using our anelastic splitting functions. This model will greatly help in the interpretation of the anelastic splitting function measurements, and test if, for example, what we see in the lower mantle sensitive modes is not due transition zone structure instead. Making such a model should be done using two different methods:

(i) least-squares inversion (e.g. Koelemeijer et al., 2016), and

(ii) Hamiltonian Monte Carlo inversion (e.g. Fichtner et al., 2019; van Tent et al., 2020) in order to get proper uncertainties.

- In addition to making a mantle model, we also would like to construct a new global tomographic model of inner core 3D attenuation using the available inner core anelastic splitting functions (e.g. Mäkinen and Deuss, 2013; Pachhai et al., 2020), in combination with compressional body wave observations.

- We found that mode ${ }_{0} S_{2}$ is very important in constraining both density (e.g. Moulik and Ekström, 2016) and attenuation (Section 6.3.1). Unfortunately, current measurements are not very well constrained and have large uncertainties due to lack of data. This situation may improve by observing splitting functions of long-period normal modes (e.g. ${ }_{0} S_{2}$ ) using superconducting gravimeter
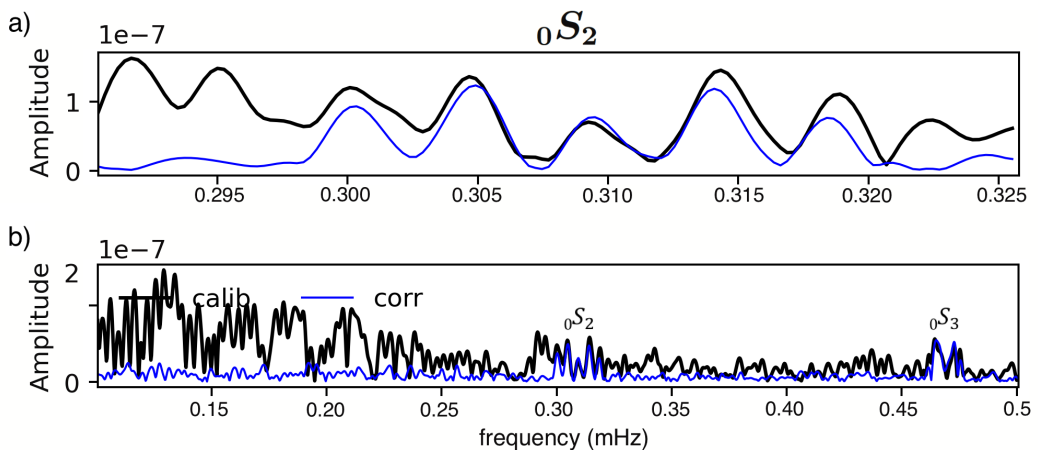

Figure 7.1: Vertical component superconducting gravimeter data of the $M_{w}$ 8.4 Peru earthquake of 2001 for station Strasbourg (ST) with a time window of 0-242 hrs. Observed spectral amplitude of the calibrated data (black) is compared to the observed spectral amplitude of the calibrated and pressure corrected data (blue, Zürn and Widmer-Schnidrig, 1995). (a) Mode ${ }_{0} S_{2}$ and (b) 0.1-0.5 mHz frequency band. 
data (Fig. 7.1, e.g. Häfner and Widmer-Schnidrig, 2013) together with broadband seismometer data. We plan to do this in collaboration with Rudolf Widmer-Schnidrig.

- Our interesting, but also counterintuitive, observation of weak attenuation in the LLSVPs and strong attenuation in the 'ring around the Pacific' requires further work to interpret its mineralogical cause. We would like to extend our interpretation of the $3 \mathrm{D}$ variations in attenuation in the mantle and inner core, through collaborations with mineral physicists, such as Laura Cobden and Ulrich H. Faul, with a focus on

(i) The role of temperature and grain-size in the key regions shown to have strong or weak attenuation in our normal mode anelastic splitting function measurements. Fig. 7.2, shows the initial results from a collaboration with Laura Cobden and Ulrich H. Faul using the laboratory-based viscoelastic 'Extended Burgers Model' (EBM, Jackson and Faul, 2010b);

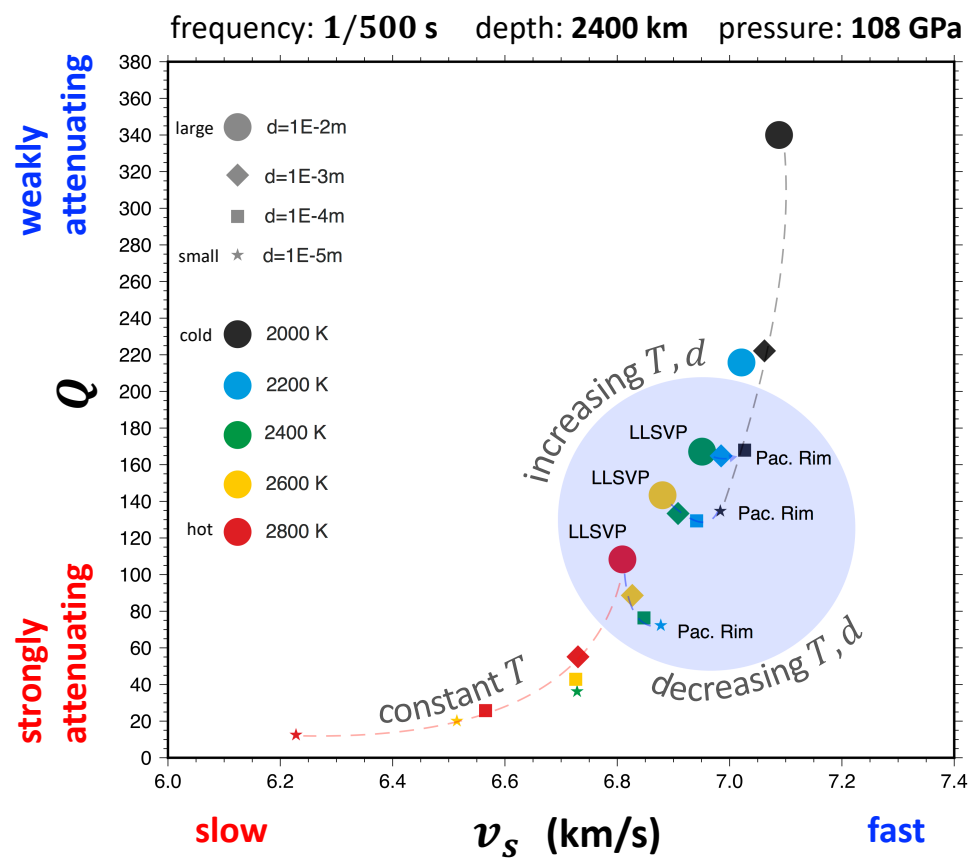

Figure 7.2: Figure by Laura Cobden, personal communication. Preliminary relationship between $v_{s}$ and $q_{\mu}$ with varying temperature $T$ and grain-size $d$, at $2 \mathrm{mHz}$ and $2400 \mathrm{~km}$ using the laboratory-based viscoelastic 'Extended Burgers Model' (EBM, Jackson and Faul, 2010b), and the estimated lower mantle parameters of Dannberg et al. (2017) and Lau and Faul (2019). The blue circle indicates the temperature and grain-size ranges where $v_{s}$ and $q_{\mu}$ increase as temperature and grain-size decrease (blue dashed lines). The black and red dashed lines indicate $v_{s}$ and $q_{\mu}$ changes at constant temperature $(2000 \mathrm{~K}, 2800 \mathrm{~K})$ with varying grain-size. 
(ii) The possible implications for attenuation of the perovskite to postperovskite phase transition in the lower mantle from ab-initio molecular simulations (e.g. Goryaeva et al., 2016); and

(iii) The source of the discrepancy between ab-initio molecular simulations and seismic measurements of inner core shear velocity (e.g. Deuss, 2008).

- Finally, we also realised that we have not analysed the effects of station distribution, noise, source radiation patterns, or short wavelength elastic structure on measuring anelastic splitting functions, in any of our tests including synthetic spectra analysis. We will also test for these effects, in particular

(i) There might be an influence of the distribution of the chosen networks and stations on our elastic and anelastic measurements, which we should also evaluate further using our synthetic test method (e.g. Majstorović et al., 2019).

(ii) Defining and including noise in synthetic test analysis, as well as further evaluating the level of noise in our real data is important to determine potential, significant trade-offs between noise and our 3D anelastic observations (e.g. Resovsky and Ritzwoller, 1998).

(iii) In addition, the uncertainties in the scalar moment of earthquakes, source radiation patterns can also trade-off with our 3D anelastic observations, and tests evaluating its effect on 3D attenuation should be performed (e.g. Romanowicz and Mitchell, 2015)

(iv) Lastly, the possible effects of short wavelength elastic structure on our long wavelength normal mode 3D anelastic observations should also be the subject of future research (e.g. Romanowicz, 1987). 



\section{Bibliography}

Abramowitz, M. and I. A. Stegun (1965). Handbook of mathematical functions with formulas, graphs and mathematical tables. Dover Books on Advanced Mathematics, New York.

Adenis, A.; E. Debayle, and Y. Ricard (2017). Attenuation tomography of the upper mantle. Geophysical Research Letters 44.15, pp. 77157724 .

Ahrens, J.; B. Geveci, and C. Law (2005). Paraview: An end-user tool for large data visualization. The visualization handbook 717.8 .

Aizawa, Y.; A. Barnhoorn; U. H. Faul, et al. (2008). Seismic properties of Anita Bay dunite: An exploratory study of the influence of water. Journal of Petrology 49.4, pp. 841-855.

Akbarashrafi, F; D Al-Attar; A. Deuss; J. Trampert, and A. P. Valentine (2017). Exact free oscillation spectra, splitting functions and the resolvability of Earth's density structure. Geophysical Journal International 213.1, pp. 5876.

Akbarashrafi, F. (2020). Resolvability of the 3D density structure of the Earth's mantle using normal mode theory. PhD thesis. Utrecht University Dept. of Earth Sciences.

Anderson, D. L. and J. W. Given (1982). Absorption band $Q$ model for the Earth. Journal of Geophysical Research: Solid Earth 87.B5, pp. 3893-3904.

Anderson, D. L. and R. S. Hart (1978). $Q$ of the Earth. Journal of Geophysical Research: Solid Earth 83.B12, pp. 5869-5882.
Anderson, D. L. and J. B. Minster (1979). The frequency dependence of $Q$ in the Earth and implications for mantle rheology and Chandler wobble. Geophysical Journal International 58.2, pp. 431-440.

Andrews, J; A Deuss, and J. H. Woodhouse (2006). Coupled normal-mode sensitivity to inner-core shear velocity and attenuation. Geophysical Journal International 167.1, pp. 204-212.

Bao, X.; C. A. Dalton, and J. Ritsema (2016). Effects of elastic focusing on global models of Rayleigh wave attenuation. Geophysical Supplements to the Monthly Notices of the Royal Astronomical Society 207.2, pp. 1062-1079.

Barnhoorn, A; I. Jackson; J. Fitz Gerald; A. Kishimoto, and K Itatani (2016). Grain sizesensitive viscoelastic relaxation and seismic properties of polycrystalline MgO. Journal of Geophysical Research: Solid Earth 121.7, pp. 4955-4976.

Beghein, C. and J. Trampert (2003). Robust normal mode constraints on inner-core anisotropy from model space search. Science 299.5606, pp. 552-555.

Belonoshko, A. B.; N. V. Skorodumova; S. Davis, et al. (2007). Origin of the low rigidity of the Earth's inner core. Science 316.5831, pp. 1603 1605 . 
Belonoshko, A. B.; T. Lukinov; J. Fu, et al. (2017). Stabilization of body-centred cubic iron under inner-core conditions. Nature Geoscience 10.4, pp. 312-316.

Belonoshko, A. B.; J. Fu; T. Bryk; S. I. Simak, and M. Mattesini (2019). Low viscosity of the Earth's inner core. Nature communications 10.1 , p. 2483.

Benjamin, D.; J. Wahr; R. D. Ray; G. D. Egbert, and S. D. Desai (2006). Constraints on mantle anelasticity from geodetic observations, and implications for the $J_{2}$ anomaly. Geophysical Journal International 165.1, pp. 3-16.

Beyreuther, M.; R. Barsch; L. Krischer, et al. (2010). ObsPy: A Python toolbox for seismology. Seismological Research Letters 81.3, pp. 530-533.

Bhattacharyya, J.; G. Masters, and P. M. Shearer (1996). Global lateral variations of shear wave attenuation in the upper mantle. Journal of Geophysical Research: Solid Earth 101.B10, pp. 22273-22289.

Birch, A. F. (1940). The alpha-gamma transformation of iron at high pressures, and the problem of the Earth's magnetism. American Journal of Science 238.3, pp. 192-211.

Birch, F. (1952). Elasticity and constitution of the Earth's interior. Journal of Geophysical Research 57.2, pp. 227-286.

Bird, P. (2003). An updated digital model of plate boundaries. Geochemistry, Geophysics, Geosystems 4.3 .

Brett, H. and A. Deuss (2020). Inner core anisotropy measured using new ultra-polar PKIKP paths. Geophysical Journal International 223.2, pp. 1230-1246.

Buffett, B. A. and H. Wenk (2001). Texturing of the Earth's inner core by Maxwell stresses. Nature 413.6851, pp. 60-63.

Buland, R; J Berger, and F. Gilbert (1979). Observations from the IDA network of attenuation and splitting during a recent Earthquake. Nature 277, pp. 358-362.

Bullen, K. E. (1946). A hypothesis on compressibility at pressures of the order of a million atmospheres. Nature 157.3987, p. 405.
Canas, J. A. and B. J. Mitchell (1978). Lateral variation of surface-wave anelastic attenuation across the Pacific. Bulletin of the Seismological Society of America 68.6, pp. 1637-1650.

Cao, A.; B. Romanowicz, and N. Takeuchi (2005). An observation of PKJKP: Inferences on inner core shear properties. Science 308.5727, pp. 1453-1455.

Cormier, V. F. (1981). Short-period PKP phases and the anelastic mechanism of the inner core. Physics of the Earth and Planetary Interiors 24.4, pp. 291-301.

Cottaar, S. and B. Buffett (2012). Convection in the Earth's inner core. Physics of the Earth and Planetary Interiors 198, pp. 67-78.

Creasy, N.; A. Pisconti; M. D. Long; C. Thomas, and J. Wookey (2019). Constraining lowermost mantle anisotropy with body waves: A synthetic modelling study. Geophysical Journal International 217.2, pp. 766-783.

Creasy, N.; J. Girard; J. O. Eckert Jr, and K. K. M. Lee (2020). The Role of Redox on Bridgmanite Crystal Chemistry and Calcium Speciation in the Lower Mantle. Journal of Geophysical Research: Solid Earth, e2020JB020783.

Dahlen, F. A. (1968). The normal modes of a rotating, elliptical Earth. Geophysical Journal International 16.4, pp. 329-367.

Dahlen, F. A. (1982). The effect of data windows on the estimation of free oscillation parameters. Geophysical Journal International 69.2, pp. 537-549.

Dahlen, F. A. and R. V. Sailor (1979). Rotational and elliptical splitting of the free oscillations of the Earth. Geophysical Journal International 58.3, pp. 609-623.

Dahlen, F. A. and J. Tromp (1998). Theoretical global seismology. Princeton University Press.

Dalton, C. A.; G. Ekström, and A. M. Dziewonski (2008). The global attenuation structure of the upper mantle. Journal of Geophysical Research: Solid Earth 113.B9.

Dalton, C. A.; G. Ekström, and A. M. Dziewonski (2009). Global seismological shear velocity and attenuation: A comparison with experimental observations. Earth and Planetary Science Letters 284.1, pp. 65-75. 
Dannberg, J; Z Eilon; U. H. Faul, et al. (2017). The importance of grain-size to mantle dynamics and seismological observations. Geochemistry, Geophysics, Geosystems 18.8, pp. 3034 3061.

Davaille, A. and B. Romanowicz (2020). Deflating the LLSVPs: Bundles of Mantle Thermochemical Plumes Rather Than Thick Stagnant 'Piles'. Tectonics 39.10, e2020TC006265.

De Wit, R. W. L.; P. J. Käufl; A. P. Valentine, and J. Trampert (2014). Bayesian inversion of free oscillations for Earth's radial (an)elastic structure. Physics of the Earth and Planetary Interiors 237, pp. 1-17.

Deuss, A. (2008). Normal mode constraints on shear and compressional wave velocity of the Earth's inner core. Earth and Planetary Science Letters 268.3-4, pp. 364-375.

Deuss, A. (2014). Heterogeneity and anisotropy of Earth's inner core. Annual Review of Earth and Planetary Sciences 42, pp. 103-126.

Deuss, A. and J. H. Woodhouse (2001). Theoretical free-oscillation spectra: The importance of wide band coupling. Geophysical Journal International 146.3, pp. 833-842.

Deuss, A.; J. H. Woodhouse; H. Paulssen, and J. Trampert (2000). The observation of inner core shear waves. Geophysical Journal International 142.1, pp. 67-73.

Deuss, A.; J. C. E. Irving, and J. H. Woodhouse (2010). Regional variation of inner core anisotropy from seismic normal mode observations. Science 328.5981, pp. 1018-1020.

Deuss, A.; J. Ritsema, and H. J. van Heijst (2011). Splitting function measurements for Earth's longest period normal modes using recent large Earthquakes. Geophysical Research Letters 38.4 .

Deuss, A.; J. Ritsema, and H. J. van Heijst (2013). A new catalogue of normalmode splitting function measurements up to $10 \mathrm{mHz}$. Geophysical Journal International 193.2, pp. 920-937.

Doornbos, D. J. (1983). Observable effects of the seismic absorption band in the Earth. Geophysical Journal International 75.3, pp. 693711.
Dratler, J; W. Farrell; B Block, and F. Gilbert (1971). High-Q Overtone Modes of the Earth. Geophysical Journal International 23.4, pp. 399-410.

Durek, J. J. and G. Ekström (1995). Evidence of bulk attenuation in the asthenosphere from recordings of the Bolivia Earthquake. Geophysical Research Letters 22.16, pp. 2309 2312 .

Durek, J. J. and G. Ekström (1996). A radial model of anelasticity consistent with longperiod surface-wave attenuation. Bulletin of the Seismological Society of America 86.1A, pp. 144-158.

Durek, J. J. and B. Romanowicz (1999). Inner core anisotropy inferred by direct inversion of normal mode spectra. Geophysical Journal International 139.3, pp. 599-622.

Dziewonski, A. M. and D. L. Anderson (1981). Preliminary Reference Earth Model. Physics of the Earth and Planetary Interiors 25.4, pp. 297-356.

Dziewonski, A. M. and F. Gilbert (1971). Solidity of the inner core of the Earth inferred from normal mode observations. Nature 234.5330, p. 465 .

Dziewonski, A. M. and F. Gilbert (1972). Observations of normal modes from 84 recordings of the Alaskan Earthquake of 1964 March 28. Geophysical Journal of the Royal Astronomical Society 27.4, pp. 393-446.

Dziewonski, A. M. and F. Gilbert (1973). Observations of normal modes from 84 recordings of the Alaskan Earthquake of 1964 March 28th. Further remarks based on new spheroidal overtone data. Geophysical Journal International 35.4, pp. 401-437.

Dziewonski, A. M. and J. M. Steim (1982). Dispersion and attenuation of mantle waves through waveform inversion. Geophysical Journal International 70.2, pp. 503-527.

Dziewonski, A. M.; T. Chou, and J. H. Woodhouse (1981). Determination of earthquake source parameters from waveform data for studies of global and regional seismicity. Journal of Geophysical Research: Solid Earth 86.B4, pp. 2825-2852. 
Dziewonski, A. M.; V. Lekić, and B. Romanowicz (2010). Mantle anchor structure: an argument for bottom up tectonics. Earth and Planetary Science Letters 299.1-2, pp. 69-79.

Dziewonski, A. M.; G. Ekström, and M Nettles (2012). The global CMT project 2004-2010: Centroid-moment tensors for 13,017 earthquakes. Physics of the Earth and Planetary Interiors 200, pp. 1-9.

Edmonds, A. R. (1960). Angular momentum in quantum mechanics. Princeton University Press.

Faul, U. H. and I. Jackson (2005). The seismological signature of temperature and grain size variations in the upper mantle. Earth and Planetary Science Letters 234.1-2, pp. 119 134.

Faul, U. H. and I. Jackson (2015). Transient creep and strain energy dissipation: An experimental perspective. Annual Review of Earth and Planetary Sciences 43, pp. 541-569.

Faul, U. H.; J. D. Fitz Gerald, and I. Jackson (2004). Shear wave attenuation and dispersion in melt-bearing olivine polycrystals: 2 . Microstructural interpretation and seismological implications. Journal of Geophysical Research: Solid Earth 109.B6.

Fichtner, A.; A. Zunino, and L. Gebraad (2019). Hamiltonian Monte Carlo solution of tomographic inverse problems. Geophysical Journal International 216.2, pp. 1344-1363.

French, S. W. and B. Romanowicz (2014). Wholemantle radially anisotropic shear velocity structure from spectral-element waveform tomography. Geophysical Journal International 199.3, pp. 1303-1327.

French, S. W.; V. Lekić, and B. Romanowicz (2013). Waveform tomography reveals channeled flow at the base of the oceanic asthenosphere. Science 342.6155, pp. 227-230.

Garnero, E. J.; A. K. McNamara, and S. Shim (2016). Continent-sized anomalous zones with low seismic velocity at the base of Earth's mantle. Nature Geoscience 9.7, pp. 481-489.

Giardini, D.; X.-D. Li, and J. H. Woodhouse (1988). Splitting functions of long-period normal modes of the Earth. Journal of Geophysical Research: Solid Earth 93.B11, pp. 13716 13742 .
Gilbert, F. (1971). The diagonal sum rule and averaged eigenfrequencies. Geophysical Journal International 23.1, pp. 119-123.

Gilbert, F. and A. M. Dziewonski (1975). An application of normal mode theory to the retrieval of structural parameters and source mechanisms from seismic spectra. Philosophical Transactions of the Royal Society of London. Series A, Mathematical and Physical Sciences 278.1280, pp. 187-269.

Goryaeva, A. M.; P. Carrez, and P. Cordier (2016). Low viscosity and high attenuation in $\mathrm{MgSiO}_{3}$ post-perovskite inferred from atomic-scale calculations. Scientific Reports 6, p. 34771.

Gribb, T. T. and R. F. Cooper (1998). Lowfrequency shear attenuation in polycrystalline olivine: Grain boundary diffusion and the physical significance of the Andrade model for viscoelastic rheology. Journal of Geophysical Research: Solid Earth 103.B11, pp. 2726727279 .

Gung, Y and B. Romanowicz (2004). $Q$ tomography of the upper mantle using threecomponent long-period waveforms. Geophysical Journal International 157.2, pp. 813-830.

Häfner, R and R Widmer-Schnidrig (2013). Signature of 3-D density structure in spectra of the spheroidal free oscillation ${ }_{0} S_{2}$. Geophysical Journal International 192.1, pp. 285-294.

Hammond, W. C. and E. D. Humphreys (2000a). Upper mantle seismic wave velocity: Effects of realistic partial melt geometries. Journal of Geophysical Research: Solid Earth 105.B5, pp. 10975-10986.

Hammond, W. C. and E. D. Humphreys (2000b). Upper mantle seismic wave attenuation: Effects of realistic partial melt distribution. Journal of Geophysical Research: Solid Earth 105.B5, pp. 10987-10999.

Harmon, N.; D. W. Forsyth, and D. S. Weeraratne (2009). Thickening of young Pacific lithosphere from high-resolution Rayleigh wave tomography: A test of the conductive cooling model. Earth and Planetary Science Letters 278.1-2, pp. 96-106.

He, X. and J. Tromp (1996). Normal-mode constraints on the structure of the Earth. Journal of Geophysical Research: Solid Earth 101.B9, pp. 20053-20082. 
Hjörleifsdóttir, V. and G. Ekström (2010). Effects of three-dimensional Earth structure on CMT earthquake parameters. Physics of the Earth and Planetary Interiors 179.3-4, pp. 178-190.

Hrubiak, R.; Y. Meng, and G. Shen (2018). Experimental evidence of a body centered cubic iron at the Earth's core condition. arXiv preprint arXiv:1804.05109.

Hwang, Y. K. and J. Ritsema (2011). Radial $Q_{\mu}$ structure of the lower mantle from teleseismic body-wave spectra. Earth and Planetary Science Letters 303.3-4, pp. 369-375.

Irving, J. C. E.; A Deuss, and J Andrews (2008). Wide-band coupling of Earth's normal modes due to anisotropic inner core structure. Geophysical Journal International 174.3, pp. 919929 .

Ishii, M. and J. Tromp (1999). Normal-Mode and Free-Air Gravity Constraints on Lateral Variations in Velocity and Density of Earth's Mantle. Science 285.5431, pp. 1231-1236.

Jackson, I. (2015). 2.21 - Properties of rocks and minerals: Physical origins of anelasticity and attenuation in rock. Treatise on Geophysics 2, pp. 539-571.

Jackson, I. and U. H. Faul (2010a). Grain sizesensitive viscoelastic relaxation in olivine: Towards a robust laboratory-based model for seismological application. Physics of the Earth and Planetary Interiors 183.1-2, pp. 151-163.

Jackson, I. and U. H. Faul (2010b). Grain sizesensitive viscoelastic relaxation in olivine: Towards a robust laboratory-based model for seismological application. Physics of the Earth and Planetary Interiors 183.1-2, pp. 151-163.

Jackson, I.; J. D. F. Gerald, and H. Kokkonen (2000). High-temperature viscoelastic relaxation in iron and its implications for the shear modulus and attenuation of the Earth's inner core. Journal of Geophysical Research: Solid Earth 105.B10, pp. 23605-23634.

Jackson, I.; U. H. Faul; J. D. Fitz Gerald, and B. H. Tan (2004). Shear wave attenuation and dispersion in melt-bearing olivine polycrystals: 1. Specimen fabrication and mechanical testing. Journal of Geophysical Research: Solid Earth 109.B6.
Jagt, L. and A. Deuss (2021). Comparing onestep full-spectrum inversion with two-step splitting function inversion in normal mode tomography. under review: Geophysical Journal International.

Jeanloz, R. and H. Wenk (1988). Convection and anisotropy of the inner core. Geophysical Research Letters 15.1, pp. 72-75.

Kanamori, H. and D. L. Anderson (1977). Importance of physical dispersion in surface wave and free oscillation problems. Reviews of Geophysics 15.1, pp. 105-112.

Karaoğlu, H. and B. Romanowicz (2018). Inferring global upper-mantle shear attenuation structure by waveform tomography using the spectral element method. Geophysical Journal International 213.3, pp. 1536-1558.

Karato, S. (1993). Inner core anisotropy due to the magnetic field - induced preferred orientation of iron. Science 262.5140, pp. 1708 1711.

Karato, S. (1999). Seismic anisotropy of the Earth's inner core resulting from flow induced by Maxwell stresses. Nature 402.6764, pp. $871-$ 873.

Karato, S. (2003). Mapping water content in upper mantle. Geophysical MonographAmerican Geophysical Union 138, pp. 135152.

Karato, S. (2008). Deformation of Earth materials. An Introduction to the Rheology of Solid Earth 463.

Karato, S. and H. Jung (1998). Water, partial melting and the origin of the seismic low velocity and high attenuation zone in the upper mantle. Earth and Planetary Science Letters 157.3-4, pp. 193-207.

Knopoff, L; P. A. Rydelek; W Zürn, and T Yogi (1979). $Q$ of mode ${ }_{0} S_{0}$. Journal of Geophysics / IF 32.18 46.1, pp. 89-95.

Knopoff, L. (1964). Solid-earth geophysics. $Q$ : Reviews of Geophysics 2, pp. 625-660.

Koelemeijer, P.; A. Deuss, and J. Ritsema (2013). Observations of core-mantle boundary Stoneley modes. Geophysical Research Letters 40.11, pp. 2557-2561. 
Koelemeijer, P.; J. Ritsema; A. Deuss, and H. J. van Heijst (2016). SP12RTS: A degree-12 model of shear-and compressional-wave velocity for Earth's mantle. Geophysical Journal International 204.2, pp. 1024-1039.

Koelemeijer, P.; A. Deuss, and J. Ritsema (2017). Density structure of Earth's lowermost mantle from Stoneley mode splitting observations. Nature Communications 8, p. 15241.

Konishi, K.; N. Fuji, and F. Deschamps (2020). Three-dimensional Elastic and Anelastic Structure of the Lowermost Mantle Beneath the Western Pacific From Finite-Frequency Tomography. Journal of Geophysical Research: Solid Earth 125.2, e2019JB018089.

Kuo, C. and B. Romanowicz (2002). On the resolution of density anomalies in the Earth's mantle using spectral fitting of normalmode data. Geophysical Journal International 150.1, pp. 162-179.

Kustowski, B; G. Ekström, and A. M. Dziewoński (2008). Anisotropic shear-wave velocity structure of the Earth's mantle: A global model. Journal of Geophysical Research: Solid Earth 113.B6.

Laske, G. and R. Widmer-Schnidrig (2007). Theory and observations-normal modes and surface wave measurements. Treatise on Geophysics 1, pp. 67-125.

Laske, G.; G. Masters, and A. M. Dziewonski (2001). New measurements of radial mode eigenfrequencies. AGU Fall Meeting Abstracts 2001, S32B-0629.

Laske, G.; A. M. Dziewonski, and G. Masters (2005). The Reference Earth Model website. URL: http://mahi.ucsd.edu/Gabi/rem.html.

Lau, H. C. P. and U. H. Faul (2019). Anelasticity from seismic to tidal timescales: Theory and observations. Earth and Planetary Science Letters 508, pp. 18-29.

Lau, H. C. P.; J. X. Mitrovica; J. L. Davis, et al. (2017). Tidal tomography constrains Earth's deep-mantle buoyancy. Nature 551.7680, p. 321.

Lawrence, J. F. and M. E. Wysession (2006a). QLM9: A new radial quality factor $\left(Q_{\mu}\right)$ model for the lower mantle. Earth and Planetary Science Letters 241.3-4, pp. 962-971.
Lawrence, J. F. and M. E. Wysession (2006b). Seismic evidence for subduction-transported water in the lower mantle. Geophysical Monograph-American Geophysical Union 168, p. 251 .

Lawrence, J. F. and M. E. Wysession (2006c). A low order whole mantle model of seismic attenuation. Unpublished.

Lay, T. and E. J. Garnero (2011). Deep mantle seismic modelling and imaging. Annual Review of Earth and Planetary Sciences 39, pp. $91-123$.

Lee, C. A. (2003). Compositional variation of density and seismic velocities in natural peridotites at STP conditions: Implications for seismic imaging of compositional heterogeneities in the upper mantle. Journal of Geophysical Research: Solid Earth 108.B9.

Lehmann, I. (1936). P', Publ. Bur. Centr. Seism. Internat. Serie A 14, pp. 87-115.

Lekić, V.; J. Matas; M. Panning, and B. Romanowicz (2009). Measurement and implications of frequency dependence of attenuation. Earth and Planetary Science Letters 282.1, pp. 285-293.

Li, X.; D. Giardini, and J. H. Woodhouse (1991). Large-scale three-dimensional evendegree structure of the Earth from splitting of long-period normal modes. Journal of Geophysical Research: Solid Earth 96.B1, pp. 551577.

Li, X. and V. F. Cormier (2002). Frequencydependent seismic attenuation in the inner core, 1. A viscoelastic interpretation. Journal of Geophysical Research: Solid Earth 107.B12, ESE-13.

Liu, H.; D. L. Anderson, and H. Kanamori (1976). Velocity dispersion due to anelasticity; implications for seismology and mantle composition. Geophysical Journal International 47.1, pp. $41-58$.

Lythgoe, K. H. and A. Deuss (2015). The existence of radial anisotropy in Earth's upper inner core revealed from seismic normal mode observations. Geophysical Research Letters 42.12, pp. 4841-4848. 
Majstorović, J.; S. Rosat; S. Lambotte, and Y. Rogister (2019). Testing performances of the optimal sequence estimation and autoregressive method in the frequency domain for estimating eigenfrequencies and zonal structure coefficients of low-frequency normal modes. Geophysical Journal International 216.2, pp. 1157-1176.

Mäkinen, A. M. and A. Deuss (2013). Normal mode splitting function measurements of anelasticity and attenuation in the Earth's inner core. Geophysical Journal International 194.1, pp. 401-416.

Mäkinen, A. M.; A. Deuss, and S. A. T. Redfern (2014). Anisotropy of Earth's inner core intrinsic attenuation from seismic normal mode models. Earth and Planetary Science Letters 404, pp. 354-364.

Martorell, B.; L. Vočadlo; J. Brodholt, and I. G. Wood (2013). Strong premelting effect in the elastic properties of hcp-Fe under inner-core conditions. Science 342.6157, pp. 466-468.

Masters, G. and F. Gilbert (1981). Structure of the inner core inferred from observations of its spheroidal shear modes. Geophysical Research Letters 8.6, pp. 569-571.

Masters, G. and F. Gilbert (1983). Attenuation in the Earth at low frequencies. Philosophical Transactions of the Royal Society of London. Series A, Mathematical and Physical Sciences 308.1504, pp. 479-522.

Masters, G. and P. M. Shearer (1990). Summary of seismological constraints on the structure of the Earth's core. Journal of Geophysical Research: Solid Earth 95.B13, pp. 21691-21695.

Masters, G. and R Widmer-Schnidrig (1995). Free oscillations: Frequencies and attenuations. Global Earth Physics: A handbook of physical constants 1. Ed. by T. J. Ahrens, p. 104.

Masters, G.; G. Laske, and F. Gilbert (2000a). Autoregressive estimation of the splitting matrix of free-oscillation multiplets. Geophysical Journal International 141.1, pp. 25-42.

Masters, G.; G. Laske, and F. Gilbert (2000b). Matrix autoregressive analysis of freeoscillation coupling and splitting. Geophysical Journal International 143.2, pp. 478-489.
Masters, G.; G. Laske; H. Bolton, and A. Dziewonski (2000c). The relative behaviour of shear velocity, bulk sound speed, and compressional velocity in the mantle: Implications for chemical and thermal structure. Earth's Deep Interior: Mineral physics and tomography from the atomic to the global scale 117 , pp. $63-87$.

Masters, G.; J Woodhouse, and G Freeman (2011). Mineos v1. 0.2 [software]. Computational Infrastructure for Geodynamics. URL: https : / / geodynamics . org/cig/software / mineos/.

McCarthy, C. and Y. Takei (2011). Anelasticity and viscosity of partially molten rock analogue: Toward seismic detection of small quantities of melt. Geophysical Research Letters 38.18 .

Molnar, P. and J. Oliver (1969). Lateral variations of attenuation in the upper mantle and discontinuities in the lithosphere. Journal of Geophysical Research 74.10, pp. 2648-2682.

Mooney, W. D.; G. Laske, and G. Masters (1998). CRUST5.1: A global model at 5 degrees by 5 degrees. Journal of Geophysical Research 102, pp. $727-748$.

Morelli, A.; A. M. Dziewonski, and J. H. Woodhouse (1986). Anisotropy of the inner core inferred from PKIKP travel times. Geophysical Research Letters 13.13, pp. 1545-1548.

Morozov, I. B. (2015). On the relation between bulk and shear seismic dissipation. Bulletin of the Seismological Society of America 105.6, pp. 3180-3188.

Mosca, I.; L. Cobden; A. Deuss; J. Ritsema, and J. Trampert (2012). Seismic and mineralogical structures of the lower mantle from probabilistic tomography. Journal of Geophysical Research: Solid Earth 117.B6.

Moulik, P and G. Ekström (2014). An anisotropic shear velocity model of the Earth's mantle using normal modes, body waves, surface waves and long-period waveforms. Geophysical Journal International 199.3, pp. 1713-1738.

Moulik, P and G. Ekström (2016). The relationships between large-scale variations in shear velocity, density, and compressional velocity in the Earth's mantle. Journal of Geophysical Research: Solid Earth 121.4, pp. 2737-2771. 
Murakami, M.; K. Hirose; K. Kawamura; N. Sata, and Y. Ohishi (2004). Post-perovskite phase transition in $\mathrm{MgSiO}_{3}$. Science 304.5672, pp. 855-858.

Nowacki, A.; J. Wookey, and J. Kendall (2011). New advances in using seismic anisotropy, mineral physics and geodynamics to understand deformation in the lowermost mantle. Journal of Geodynamics 52.3-4, pp. 205-228.

O'Connell, R. and B Budiansky (1978). Measures of dissipation in viscoelastic media. Geophysical Research Letters 5.1, pp. 5-8.

Oganov, A. R. and S. Ono (2004). Theoretical and experimental evidence for a postperovskite phase of $\mathrm{MgSiO}_{3}$ in Earth's D' layer. Nature 430.6998, pp. 445-448.

Okal, E. A. (1996). Radial modes from the great 1994 Bolivian earthquake: No evidence for an isotropic component to the source. Geophysical Research Letters 23.5, pp. 431-434.

Okal, E. A. and S. Stein (2009). Observations of ultra-long period normal modes from the 2004 Sumatra-Andaman Earthquake. Physics of the Earth and Planetary Interiors 175.1, pp. 53-62.

Pachhai, S.; G. Masters, and G. Laske (2020). Probabilistic estimation of structure coefficients and their uncertainties, for innercore sensitive modes, using matrix autoregression. Geophysical Journal International 221.2, pp. 1366-1383.

Park, J. (1990). Radial mode observations from the 5/23/89 Macquarie Ridge Earthquake. Geophysical Research Letters 17.7, pp. 10051008.

Reid, F. J. L.; J. H. Woodhouse, and H. J. van Heijst (2001). Upper mantle attenuation and velocity structure from measurements of differential S phases. Geophysical Journal International 145.3, pp. 615-630.

Resovsky, J. S. and M. H. Ritzwoller (1998). New and refined constraints on three-dimensional Earth structure from normal modes below 3 mHz. Journal of Geophysical Research: Solid Earth 103.B1, pp. 783-810.

Resovsky, J.; J. Trampert, and R. D. van der Hilst (2005). Error bars for the global seismic $Q$ profile. Earth and Planetary Science Letters 230.3, pp. 413-423.
Riedesel, M. A.; D. Agnew; J. Berger, and F. Gilbert (1980). Stacking for the frequencies and $Q$ s of ${ }_{0} S_{0}$ and ${ }_{1} S_{0}$. Geophysical Journal International 62.2, pp. 457-471.

Ritsema, J.; H. J. van Heijst, and J. H. Woodhouse (1999). Complex shear wave velocity structure imaged beneath Africa and Iceland. Science 286.5446, pp. 1925-1928.

Ritsema, J.; A. Deuss; H. J. van Heijst, and J. H. Woodhouse (2011). S40RTS: A degree-40 shear-velocity model for the mantle from new Rayleigh wave dispersion, teleseismic traveltime and normal-mode splitting function measurements. Geophysical Journal International 184.3, pp. 1223-1236.

Ritzwoller, M.; G. Masters, and F. Gilbert (1986). Observations of anomalous splitting and their interpretation in terms of aspherical structure. Journal of Geophysical Research: Solid Earth 91.B10, pp. 10203-10228.

Robson, A. J. S. and B. Romanowicz (2019). New normal mode constraints on bulk inner core velocities and density. Physics of the Earth and Planetary Interiors, p. 106310.

Romanowicz, B and B Mitchell (2015). 1.25 - Deep Earth structure: $Q$ of the Earth from crust to core. Treatise on Geophysics 1, pp. 789-827.

Romanowicz, B. (1987). Multiplet-multiplet coupling due to lateral heterogeneity: Asymptotic effects on the amplitude and frequency of the Earth's normal modes. Geophysical Journal International 90.1, pp. 75-100.

Romanowicz, B. (1990). The upper mantle degree 2: Constraints and inferences from global mantle wave attenuation measurements. Journal of Geophysical Research: Solid Earth 95.B7, pp. 11051-11071.

Romanowicz, B. (1995). A global tomographic model of shear attenuation in the upper mantle. Journal of Geophysical Research: Solid Earth 100.B7, pp. 12375-12394.

Romanowicz, B. (1998). Attenuation tomography of the Earth's mantle: a review of current status. Pure and Applied Geophysics 153.2, pp. 257-272.

Romanowicz, B. (2001). Can we resolve 3D density heterogeneity in the lower mantle? Geophysical Research Letters 28.6, pp. 1107-1110. 
Romanowicz, B. and L. Bréger (2000). Anomalous splitting of free oscillations: A reevaluation of possible interpretations. Journal of Geophysical Research: Solid Earth 105.B9, pp. 21559-21578.

Romanowicz, B. and J. J. Durek (2000). Seismological constraints on attenuation in the Earth: A review. Earth's Deep Interior: Mineral physics and tomography from the atomic to the global scale 117. Ed. by S. Karato; A. Forte; R. Liebermann; G. Masters, and L. Stixrude, pp. 161-179.

Romanowicz, B. and Y. Gung (2002). Superplumes from the core-mantle boundary to the lithosphere: Implications for heat flux. Science 296.5567, pp. 513-516.

Romanowicz, B. and H. Wenk (2017). Anisotropy in the deep Earth. Physics of the Earth and Planetary Interiors 269, pp. 58-90.

Romanowicz, B.; G. Roult, and T. Kohl (1987). The upper mantle degree two pattern: Constraints from GEOSCOPE fundamental spheroidal mode eigenfrequency and attenuation measurements. Geophysical Research Letters 14.12, pp. 1219-1222.

Romanowicz, B.; X. Li, and J. Durek (1996). Anisotropy in the inner core: Could it be due to low-order convection? Science 274.5289, pp. 963-966.

Rosat, S.; S. Watada, and T. Sato (2007). Geographical variations of the ${ }_{0} S_{0}$ normal mode amplitude: Predictions and observations after the Sumatra-Andaman earthquake. Earth, Planets and Space 59.4, pp. 307-311.

Rost, S.; E. J. Garnero; Q. Williams, and M. Manga (2005). Seismological constraints on a possible plume root at the core-mantle boundary. Nature 435.7042, pp. 666-669.

Roult, G; B. Romanowicz, and J.-P. Montagner (1990). 3-D upper mantle shear velocity and attenuation from fundamental mode free oscillation data. Geophysical Journal International 101.1, pp. 61-80.

Roult, G.; J. Roch, and E. Clévédé (2010). Observation of split modes from the 26th December 2004 Sumatra-Andaman mega-event. Physics of the Earth and Planetary Interiors 179.1, pp. 45-59.
Sailor, R. V. and A. M. Dziewonski (1978). Measurements and interpretation of normal mode attenuation. Geophysical Journal International 53.3, pp. 559-581.

Sakai, T.; E. Ohtani; N. Hirao, and Y. Ohishi (2011). Stability field of the hcp-structure for $\mathrm{Fe}, \mathrm{Fe}-\mathrm{Ni}$, and Fe-Ni-Si alloys up to $3 \mathrm{Mbar}$ Geophysical Research Letters 38.9.

Sato, H.; I. S. Sacks; T. Murase; G. Muncill, and H. Fukuyama (1989). $Q_{p}$-melting temperature relation in peridotite at high pressure and temperature: Attenuation mechanism and implications for the mechanical properties of the upper mantle. Journal of Geophysical Research: Solid Earth 94.B8, pp. 10647-10661.

Schneider, S. and A. Deuss (2020). A new catalogue of toroidal-mode overtone splitting function measurements. Geophysical Journal International.

Schneider, S.; S. Talavera-Soza; L. Jagt, and A. Deuss (in prep.). FrosPy: Free oscillation Python toolbox for seismology.

Selby, N. D. and J. H. Woodhouse (2002). The $Q$ structure of the upper mantle: Constraints from Rayleigh wave amplitudes. Journal of Geophysical Research: Solid Earth 107.B5, ESE-5.

Shito, A.; S. Karato; K. N. Matsukage, and Y. Nishihara (2006). Towards mapping the threedimensional distribution of water in the upper mantle from velocity and attenuation tomography. Geophysical Monograph-American Geophysical Union 168, p. 225.

Singh, S.; M. Taylor, and J.-P. Montagner (2000). On the presence of liquid in Earth's inner core. Science 287.5462, pp. 2471-2474.

Sipkin, S. A. and T. H. Jordan (1980). Regional variation of $Q$ ScS. Bulletin of the Seismological Society of America 70.4, pp. 1071-1102.

Souriau, A. and B. Romanowicz (1996). Anisotropy in inner core attenuation: a new type of data to constrain the nature of the solid core. Geophysical Research Letters 23.1, pp. 1-4.

Souriau, A. and B. Romanowicz (1997). Anisotropy in the inner core: relation between P-velocity and attenuation. Physics of the Earth and Planetary Interiors 101.1-2, pp. 3347. 
Stein, S. and M. E. Wysession (2009). An introduction to seismology, Earthquakes, and Earth structure. John Wiley \& Sons.

Stoneley, R. (1924). Elastic waves at the surface of separation of two solids. Proceedings of the Royal Society of London. Series A, Containing Papers of a Mathematical and Physical Character 106.738, pp. 416-428.

Talavera-Soza, S. and A. Deuss (2020). Constraining 1-D inner core attenuation through measurements of strongly coupled normal mode pairs. Geophysical Journal International 223.1, pp. 612-621.

Talavera-Soza, S. and A. Deuss (2021). New measurements of long period radial modes using large earthquakes. Geophysical Journal International 224.2, pp. 1211-1224.

Tarantola, A. and B. Valette (1982). Generalized nonlinear inverse problems solved using the least squares criterion. Reviews of Geophysics 20.2, pp. 219-232.

Tateno, S.; K. Hirose; Y. Ohishi, and Y. Tatsumi (2010). The structure of iron in Earth's inner core. Science 330.6002, pp. 359-361.

Tateno, S.; K. Hirose; T. Komabayashi; H. Ozawa, and Y. Ohishi (2012). The structure of Fe-Ni alloy in Earth's inner core. Geophysical Research Letters 39.12.

Tkalčić, H. and T. Pham (2018). Shear properties of Earth's inner core constrained by a detection of $\mathrm{J}$ waves in global correlation wavefield. Science 362.6412, pp. 329-332.

Torsvik, T. H.; R. van der Voo; P. V. Doubrovine, et al. (2014). Deep mantle structure as a reference frame for movements in and on the Earth. Proceedings of the National Academy of Sciences 111.24, pp. 8735-8740.

Trampert, J.; F. Deschamps; J. Resovsky, and D. Yuen (2004). Probabilistic tomography maps chemical heterogeneities throughout the lower mantle. Science 306.5697, pp. 853-856.

Tromp, J. (1993). Support for anisotropy of the Earth's inner core from free oscillations. $\mathrm{Na}$ ture 366.6456, pp. 678-681.

Tsuchiya, T.; J. Tsuchiya; K. Umemoto, and R. M. Wentzcovitch (2004). Phase transition in $\mathrm{MgSiO}_{3}$ perovskite in the Earth's lower mantle. Earth and Planetary Science Letters 224.3-4, pp. 241-248.
Van Tent, R.; A. Deuss; A. Fichtner; L. Gebraad, and J. Trampert (2020). An analysis of normalmode based 3-D mantle density models using Hamiltonian Monte Carlo methods. AGU Fall Meeting Abstracts 2020, pp. DI005-03.

Van der Meer, D. G.; D. J. J. van Hinsbergen, and W. Spakman (2018). Atlas of the underworld: Slab remnants in the mantle, their sinking history, and a new outlook on lower mantle viscosity. Tectonophysics 723, pp. 309-448.

Vočadlo, L. (2007). Ab-initio calculations of the elasticity of iron and iron alloys at inner core conditions: Evidence for a partially molten inner core? Earth and Planetary Science Letters 254.1-2, pp. 227-232.

Vočadlo, L.; D. Alfè; M. J. Gillan, et al. (2003). Possible thermal and chemical stabilization of body-centred-cubic iron in the Earth's core. Nature 424.6948, p. 536.

Warren, L. M. and P. M. Shearer (2002). Mapping lateral variations in upper mantle attenuation by stacking P and PP spectra. Journal of Geophysical Research: Solid Earth 107.B12, ESE-6.

Webb, S. and I. Jackson (2003). Anelasticity and microcreep in polycrystalline $\mathrm{MgO}$ at high temperature: An exploratory study. Physics and Chemistry of Minerals 30.3, pp. 157-166.

Webb, S.; I. Jackson, and J. F. Gerald (1999). Viscoelasticity of the titanate perovskites $\mathrm{CaTiO}_{3}$ and $\mathrm{SrTiO}_{3}$ at high temperature. Physics of the Earth and Planetary Interiors 115.3-4, pp. 259-291.

Wessel, P.; W. H. F. Smith; R. Scharroo; J. Luis, and F. Wobbe (2013). Generic mapping tools: improved version released. Eos, Transactions American Geophysical Union 94.45, pp. 409410.

Widmer-Schnidrig, R (2002). Non-Linear Constraints for 1-D Elastic Core Structure from the Attenuation of ${ }_{10} S_{2}$. Annual DGG Meeting Abstracts.

Widmer-Schnidrig, R; G. Masters, and F. Gilbert (1991). Spherically symmetric attenuation within the Earth from normal mode data. Geophysical Journal International 104.3, pp. 541553. 
Woodhouse, J. H. (1980). The coupling and attenuation of nearly resonant multiplets in the Earth's free oscillation spectrum. Geophysical Journal International 61.2, pp. 261-283.

Woodhouse, J. H. (1983). The joint inversion of seismic wave for lateral variations in Earth structure and earthquake source parameters. Proc. 'Enrico Fermi' Int. Sch. Phys. LXXXV, pp. 366-397.

Woodhouse, J. H. (1988). The calculation of the eigenfrequencies and eigenfunctions of the free oscillations of the Earth and Sun. Seismological Algorithms: Computational methods and computer programs. Ed. by D. Doornbos, pp. 321-370.

Woodhouse, J. H. and F. A. Dahlen (1978). The effect of a general aspherical perturbation on the free oscillations of the Earth. Geophysical Journal International 53.2, pp. 335-354.

Woodhouse, J. H. and D. Giardini (1985). Inversion for the splitting function of isolated low order normal mode multiplets. Eos, Transactions American Geophysical Union 66, p. 300.

Woodhouse, J. H.; D. Giardini, and X. Li (1986). Evidence for inner core anisotropy from free oscillations. Geophysical Research Letters 13.13, pp. 1549-1552.

Wookey, J. and G. Helffrich (2008). Inner-core shear-wave anisotropy and texture from an observation of PKJKP waves. Nature 454.7206, p. 873 .

Yang, Y.; D. W. Forsyth, and D. S. Weeraratne (2007). Seismic attenuation near the East Pacific Rise and the origin of the low-velocity zone. Earth and Planetary Science Letters 258.1-2, pp. 260-268.
Yoshida, S.; I. Sumita, and M. Kumazawa (1996). Growth model of the inner core coupled with the outer core dynamics and the resulting elastic anisotropy. Journal of Geophysical Research: Solid Earth 101.B12, pp. 28085-28103.

Yoshino, T. and D. Yamazaki (2007). Grain growth kinetics of $\mathrm{CaIrO}_{3}$ perovskite and postperovskite, with implications for rheology of D" layer. Earth and Planetary Science Letters 255.3-4, pp. 485-493.

$\mathrm{Yu}$, S. and E. J. Garnero (2018). Ultralow velocity zone locations: A global assessment. Geochemistry, Geophysics, Geosystems 19.2, pp. 396-414.

Zhang, N.; S. Zhong; W. Leng, and Z. Li (2010). A model for the evolution of the Earth's mantle structure since the early Paleozoic. Journal of Geophysical Research: Solid Earth 115.B6.

Zhu, H.; E. Bozdağ; T. S. Duffy, and J. Tromp (2013). Seismic attenuation beneath Europe and the North Atlantic: Implications for water in the mantle. Earth and Planetary Science Letters 381, pp. 1-11.

Zürn, W and R Widmer-Schnidrig (1995). On noise reduction in vertical seismic records below $2 \mathrm{mHz}$ using local barometric pressure. Geophysical Research Letters 22.24, pp. 35373540 .

Zürn, W; L Knopoff, and P. A. Rydelek (1980). High precision measurement of the frequency of mode ${ }_{0} S_{0}$. Journal of Geophysics / IF 32.18 48.1, pp. 158-160. 



\section{Abbreviations}

\begin{tabular}{|c|c|}
\hline 1D & 1-Dimensional \\
\hline 3D & 3-Dimensional \\
\hline$\kappa$ & Bulk modulus \\
\hline$\mu$ & Shear modulus \\
\hline CMB & Core Mantle Boundary \\
\hline$c_{s t}$ & splitting function elastic structure coefficients \\
\hline$d_{s t}$ & splitting function anelastic structure coefficients \\
\hline$f_{c}$ & Center Frequency \\
\hline FC & Full-Couping \\
\hline GC & Group-Couping \\
\hline LLSVP & Large Low Shear Velocity Provinces \\
\hline$M_{0}$ & Scalar moment \\
\hline$M_{w}$ & Moment Magnitude \\
\hline PREM & Preliminary Reference Earth Model (Dziewonski and Anderson, 1981) \\
\hline $\mathbf{p P v}$ & post-Perovskite \\
\hline$q$ & Attenuation \\
\hline$q_{\kappa}$ & Bulk $(\boldsymbol{\kappa})$ Attenuation \\
\hline$q_{\mu}$ & Shear $(\boldsymbol{\mu})$ Attenuation \\
\hline$Q$ & Quality factor \\
\hline$Q_{\kappa}$ & Bulk $(\boldsymbol{\kappa})$ Quality factor \\
\hline$Q_{\mu}$ & Shear $(\boldsymbol{\mu})$ Quality factor \\
\hline QRFSI12 & $\begin{array}{l}\text { 3D attenuation } 1 / \boldsymbol{Q} \text { mantle tomographic model up-to structural degree } \mathbf{1 2} \text {, } \\
\text { derived from } \mathbf{R} \text { ayleigh-wave amplitudes, and accounting for Focussing, } \\
\text { Source and Instrument effects on the data (Dalton et al., 2008) }\end{array}$ \\
\hline $\boldsymbol{R}$ & Radius \\
\hline$R_{q}$ & Ratio relating variations in shear velocity $\delta v_{s}$ and attenuation $\delta q_{\mu}$ \\
\hline SC & Self-Couping \\
\hline S20RTS & $\begin{array}{l}\text { Shear velocity mantle tomographic model up-to structural degree } \mathbf{2 0} \text {, built } \\
\text { using Rayleigh-wave phase delays, Travel-times of teleseismic body waves } \\
\text { and the Splitting functions of normal modes (Ritsema et al., 1999) }\end{array}$ \\
\hline S40RTS & $\begin{array}{l}\text { Shear velocity mantle tomographic model up-to structural degree } \mathbf{4 0} \text {, built } \\
\text { using Rayleigh-wave phase delays, Travel-times of teleseismic body waves } \\
\text { and the Splitting functions of normal modes (Ritsema et al., 2011) }\end{array}$ \\
\hline SP12RTS & $\begin{array}{l}\text { Shear velocity and comPressional mantle tomographic model } \\
\text { up-to structural degree } \mathbf{1 2} \text {, built using Rayleigh-wave phase delays, } \\
\text { Travel-times of teleseismic body waves and the Splitting functions of } \\
\text { normal modes (Koelemeijer et al., 2016) }\end{array}$ \\
\hline$v_{s}$ & Velocity of Shear Waves \\
\hline $\boldsymbol{v}_{p}$ & Velocity of comPressional waves \\
\hline
\end{tabular}





\section{Acknowledgements Agradecimientos}

I must say I never expected to be finishing the $\mathrm{PhD}$ in the middle of a pandemic. Although this was not my first time living through one, everything I lived before pales in comparison. It definitely put the final stages of writing of the thesis into perspective, and made me appreciate the first years of the $\mathrm{PhD}$ even more. Here, I want to thank everyone that made this exciting journey possible.

First, I would like to thank my promotor Arwen Deuss. I fondly remember you welcoming me to the Netherlands, to ATUNE and to the Seismology group. Thank you for all the support and guidance, and for always sharing your eagerness to have fun doing cool science, as well as your patience teaching me all about normal modes during my PhD. I have very much enjoyed working with you and learning from you, and I'm sure I will keep doing so for the next two years.

I also want to thank my committee members Colleen Dalton, Jeroen Ritsema, Barbara Romanowicz, Jeannot Trampert and Rudolf Widmer-Schnidrig for taking the time to read my thesis and for their insightful comments and questions.

Special thanks to Ruedi for getting inspired to collaborate with me after reading my thesis. I also want to thank Laura for a very enthusiastic collaboration, that helped to put the results of my final chapter into a better context, as well as your help while I was working as a teaching assistant. I would also like to thank Heiner Igel, without whom I would not have come to Utrecht, I really appreciate all your support and advise.

Many thanks to Simon and Lisanne for taking time from their very busy last year of PhD to be my paranymphs. Dank je wel, Lisanne en vielen Dank, Simon! I could not have asked for better friends and colleagues to share the $\mathrm{PhD}$ experience. From our carefree fun start, full of late nights at the city center; to the intense middle, full of brainstorming and coffee; all the way until the stressful end, full of online therapy sessions. These last few years have been filled with Lisanne's meme faces, Simon's coffee and eggs addiction, FrosPy building, and psyduck wisdom sharing. We also had the opportunity to explore new cities $^{1}$ while at conferences, and even had the chance to go on a beautiful Canadian road trip. Unfortunately, the coronavirus

${ }^{1}$ Boston, Oxford, Washington, Edmonton, Vancouver, San Francisco, Münster, München and Sudelfeld, and hopefully many more in the future 
prevented us to visit and explore Taiwan, and even visit Yanadet in Thailand, who shared with us all his wisdom and awesome personality.

Many thanks to Theo for making sure we were always using the computer cluster responsibly, and for always having the patience to answer all of our questions and doubts. Thanks to all past and present Seismology staff members for welcoming me into the group: Arie, Henk, Jeannot, Hanneke, Ivan, Rhys, Elmer, Kabir, Haydar, Laura, Yanadet and Nesli; specially for all the coffee breaks, birthday cakes, lunches and January birthday parties full of interesting conversations and funny jokes. Thanks to Paula and Jeroen, the honorary members of the group, for always taking the time to enthusiastically listen to my research while giving constructive criticism.

Thanks to all former seismology PhD students (now living all across Europe): Nienke, Suzanne, Maria K, Sahar, Wen, Agniezska and Evangelos, for welcoming me into the Seismology group and creating a fun and lively international environment. Office mates Nienke, Maria K and Sahar made every day at the office full of dancing, exercising and tea. Special thanks to Sahar, one of my first friends here in Utrecht, for all the loud laughing contests we share every time we meet, and all the talks we have shared about life and normal modes at Jazzman, Meneer Smakers or Lebowski, and now just at home or cycling through the city. Getting to know Utrecht (specially at night) wouldn't have been the same without the usual suspects, special mention to Markus, Dan, Liesbeth, Claudia, Karin and Nevena for all those fun times. Special thanks to Pete, Tracy and Antoine for all those fun house and bar parties, and awesome celebrations.

Thanks to fellow arwenites: Annemijn, Loes, Henry and Rûna; ivans: Leon, Andreas, Haorui and David; as well as Ashim, Eldert, Janneke and Thomas. Exploring conference halls and parties, winter schools, Christmas online games, or just the FEST or a coffee break wouldn't have been half as fun without you. Special thanks to Haorui for always making us laugh with your unique humour and human perspectives, as well as your excellent taste in food. Thanks also to Rûna for always being up to have one more beer. I'm also glad to have shared the Utrecht experience with the München team: Ashim and David, and to have coincided with Sneha at many conferences during this time.

Thanks to the Amsterdamers Yuliia, Vlad, Verena, Nitish, Laura and Maria R for all those fun times at Pride, King's day or any random weekend or weekday. Laura y Maria R, empezar el doctorado no habría sido lo mismo sin ustedes dos y nuestras reuniones maratónicas en el Molino. Maria R, Holanda no sería la mitad de divertida sin vos, sin nuestras pijamadas y encuentros culinarios y políticos, ya sea en persona o por WhatsApp. Este último año lleno de coronavirus, frustraciones, burocracia y nuevos grados, no habría estado también lleno de stickers, parchís, exploding kittens, Nathy Peluso, risas y chistes sin vos.

David y Cindy, o como son conocidos internacionalmente cindavindy, gracias por todos los picnics soleados, deliciosas cenas (en especial los tamales), karaokeadas y pijamadas, y por darle el toque latino que le faltaba a Utrecht.

Gracias a los Müncheners Helisa, Saumitra, Juan Carlos, Alejandra, Adriana, Esteban y David, quienes desde el principio hicieron que la distancia a casa 


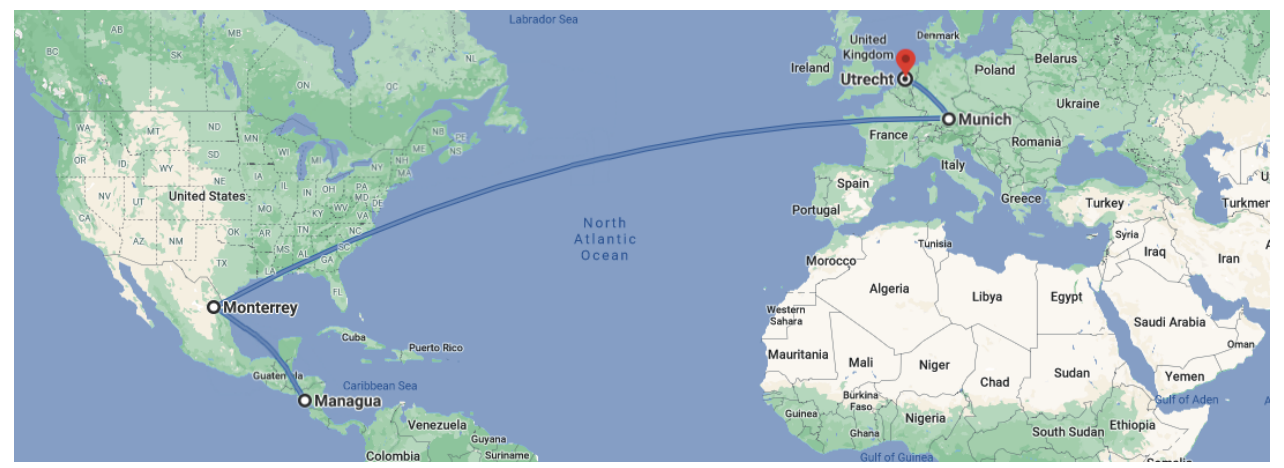

se sintiera más corta. Aunque coincidimos de manera un poco breve en München el mundo se nos hace pequeño para seguirnos viendo. Adriana, nuestro tiempo fue corto, pero inolvidable, espero que ahora que volvemos a compartir continente el coronavirus nos permita volver a vernos pronto.

Gracias a Dulce, Horacio, Pedro, María V, Pepe, Caro, Emmanuel, Lalo, Hugo, Gus, Gil, Diana, Erika, Nelson, Pepefer y Oto, quienes han hecho esta la transición del continente Americano a Europa lo más imperceptible posible. Afortunadamente, desde antes de que el coronavirus se apoderara de nuestras vidas ya hacíamos buen uso de las videollamadas y WhatsApp para ponernos al tanto de todo. La amistad puede más que $8000 \mathrm{~km} \mathrm{u} 8$ horas de distancia. Y es que a pesar de todo, durante todos estos años hemos podido seguir viéndonos en cualquier rincón del mundo en el que podíamos coincidir. Dulce y Horacio gracias por todo su apoyo y por cada aventura compartida. Pedro gracias por tus perspectivas empresariales de vida. María V gracias por siempre escucharme y estar a mi lado a pesar de todo. Espero que pronto podamos continuar con esta vida de trotamundos.

Gracias a mi familia madrileña, mis primos María Teresa, Paloma y Andrés, y mis tíos Damaris y Manolo, quienes desde México me han hecho sentir como en casa. Estas Navidades europeas no habrían sido lo mismo sin ustedes. Gracias por compartir su deliciosa comida, juegos de mesa, su calor de hogar, consejos y sabiduría.

La distancia me ha hecho apreciar aún más cada momento compartido con mi familia. Gracias a mis padres Juan y Teresa, quienes con su ejemplo y palabras me enseñaron desde pequeña que el cielo es el límite. Gracias a mi mama Yoya, quién a 10 años de su partida sigue siempre presente en todo lo que hago. Gracias a mi hermanita Adraynee, por tu paciencia y entusiasmo inacabable, y a Lourdes y María José, la familia que no sabía que necesitaba. Sin ustedes nada de esto sería posible. Gracias por su apoyo infinito e incondicional y por enseñarme todo lo que sé y todo lo que soy.

Nada en mi vida sería lo mismo sin Alberto. El cómplice de todos mis sueños y planes de respaldo. Este último año de cuarentena y tesis ha sido una montaña rusa de emociones, con una breve estancia por Barcelona, que sin vos no habría sobrevivido intacta. Este 2021 alcanzamos un título más y seguimos resolviendo el problema de los dos cuerpos, tal y como lo hemos venido haciendo durante casi ya 14 años. Gracias por compartir este y muchos otros viajes conmigo. 



\section{Curriculum Vitae}

\section{Personal Information}

DoB $28^{\text {th }}$ November of 1988 in Managua, Nicaragua

\section{Education}

2016-2021 Ph.D. in Seismology,

- Utrecht University, The Netherlands.

2014-2016 M.Sc. in Geophysics,

네 Ludwig Maximilian University and

而 Technical University of Munich, Germany.

Focused on Seismology. Outstanding master thesis award.

2006-2011 B.Sc. in Engineering Physics (cum laude),

(a) Tecnológico de Monterrey, México.

Academic scholarship granted by Tecnológico de Monterrey

\section{Experience}

\section{Academia}

2020-2023 Postdoc in Seismology,

- Utrecht University, The Netherlands.

2016 Research assistant,

* King Abdullah University of Science and Technology, Saudi Arabia.

2015 Research assistant,

цㄴ Munich GeoCenter, Germany.

Industry

2011-2014 Manufacturing engineer, to John Deere, México.

2010-2011 Manufacturing intern, 3 John Deere, México.

2010 Manufacturing intern, //L Ficosa, México. 


\section{Publications}

Talavera-Soza, Sujania and Arwen Deuss. Constraining 1-D inner core attenuation through measurements of strongly coupled normal mode pairs. Geophys. J. Int., 223(1):612-621, 2020.

Talavera-Soza, Sujania and Arwen Deuss. New measurements of long period radial modes using large earthquakes. Geophys. J. Int., 224(2):1211-1224, 2021.

Krischer, Lion, ..., Sujania Talavera-Soza, ..., Stephanie Wollherr, and Heiner Igel. seismo-live: An educational online library of Jupyter notebooks for seismology. Seismol. Res. Lett., 89(6):2413-2419, 2018.

\section{Publications in preparation}

Talavera-Soza, Sujania, Arwen Deuss, Laura Cobden, and Ulrich H Faul. Global observations of 3-D mantle attenuation using seismic normal modes.

Talavera-Soza, Sujania and Arwen Deuss. 3-D mantle attenuation using normal modes: forward modelling and sensitivity tests.

Schneider, Simon, Sujania Talavera-Soza, Lisanne Jagt, and Arwen Deuss. FrosPy: Free oscillation Python toolbox for seismology. 